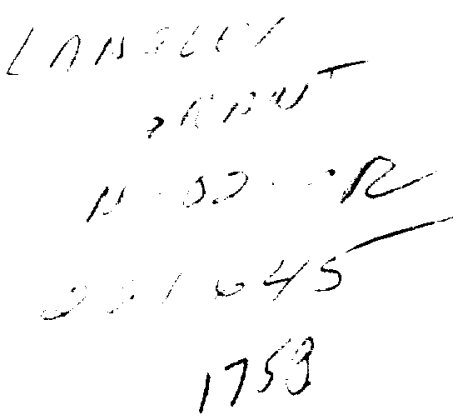

\title{
LEADING EDGE VORTEX DYNAMICS ON A PITCHING DELTA WING
}

\author{
Scott P. LeMay, Stephen M. Batill, and Robert C. Nelson \\ University of Notre Dame
}

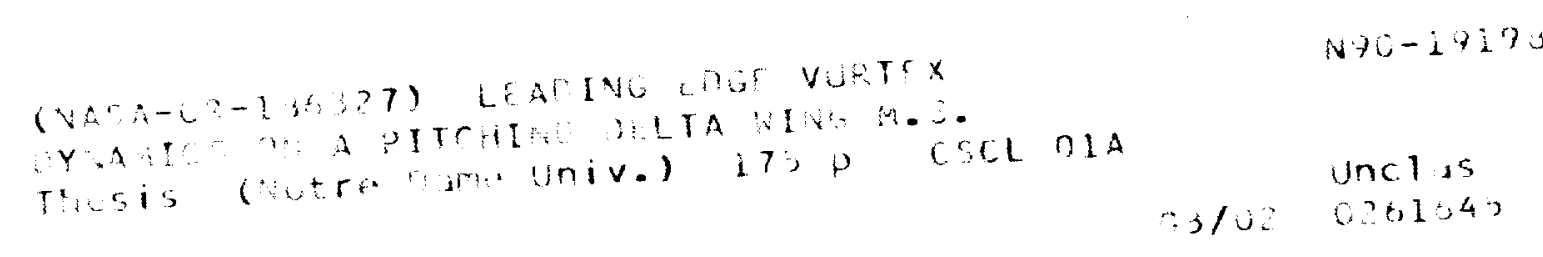

NAG-1-727

April 1988 


\title{
LEADING EDGE VORTEX DYNAMICS ON A PITCHING DELTA WING
}

\author{
A Thesis \\ Submitted to the Graduate School \\ of the University of Notre Dame \\ in Partial Fulfillment of the Requirements \\ for the Degree of \\ Master of Science \\ by
}

Scott P. LeMay, B.S.A.E.

Co-Director

Co-Director

Department of Aerospace and Mechanical Engineering

Notre Dame, Indiana

April, 1988 


\title{
LEADING EDGE VORTEX DYNAMICS \\ ON A PITCHING DELTA WING
}

\author{
Abstract \\ by \\ Scott P. LeMay
}

The leading edge flow structure was investigated on a $70^{\circ}$ flat plate delta wing which was pitched about its $1 / 2$ chord position, to increase understanding of the high angle of attack aerodynamics on an unsteady delta wing. The wing was sinusoidally pitched at reduced frequencies ranging from $\mathrm{k} \equiv 2 \pi \mathrm{fc} / \mathrm{u}=0.05$ to 0.30 at chord Reynolds numbers between 90,000 and 350,000 , for angle of attack ranges of $\alpha=29^{\circ}$ to $39^{\circ}$ and $\alpha=0^{\circ}$ to $45^{\circ}$. The wing was also impulsively pitched at an approximate rate of $0.7 \mathrm{rad} / \mathrm{s}$. During these dynamic motions, visualization of the leading edge vorticies was obtained by entraining titanium tetrachloride into the flow at the model apex.

The location of vortex breakdown was recorded using $16 \mathrm{~mm}$ high speed motion picture photography. When the wing was sinusoidally pitched, a hysteresis was observed in the location of breakdown position. This hysteresis increased with reduced frequency. The velocity of breakdown propagation along the wing, and the phase lag between model motion and breakdown location were also determined. When the wing was impulsively pitched, several convective times were required for the vortex flow to reach a steady state. Detailed information was also obtained on the oscillation of breakdown position in both static and dynamic cases. 


\section{DEDICATION}

This work is dedicated to my parents, Robert P. LeMay and Edna M.

LeMay, and also my brother Jeffery P. LeMay. Their love, support, and encouragement made it possible. 
LIST OF TABLES.

...

LIST OF FIGURES.

vi

NOMENCLATURE.

.xii

ACKNOWLEDGEMENTS.

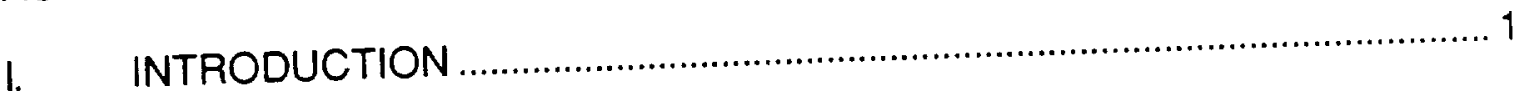

1

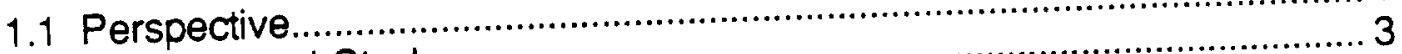

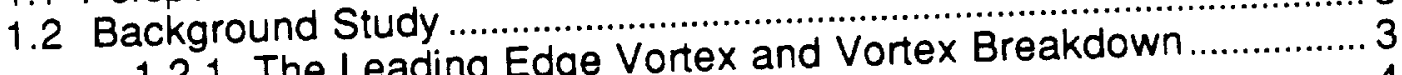

1.2.1 The Leading Edge Vortex and Vortex Breakdown................. 4

1.2.2 Unsteady Vortex Dynamics....................................................... 5

The Effect of Pitching........................................................... 8

The Effect of Plunging ..................................................10

The Effect of Unsteady Freestream Flow............................10

Static Hysteresis ..........................................................11

1.2.3 Brief Summary of Unsteady Work Reviewed........................ 12

1.3 Scope of Work

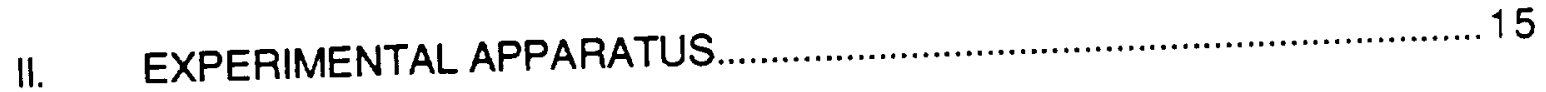

2.1 Wind Tunnel.

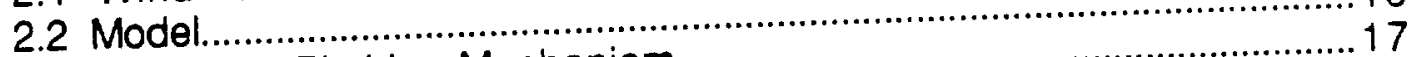

2.3 Unsteady Pitching Mechanism .................................................... 18

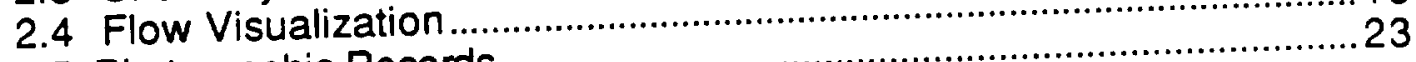

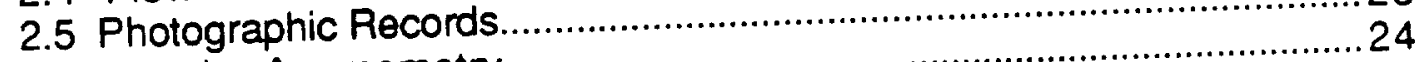

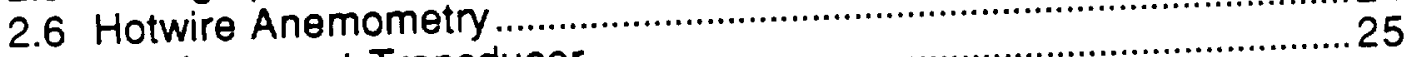

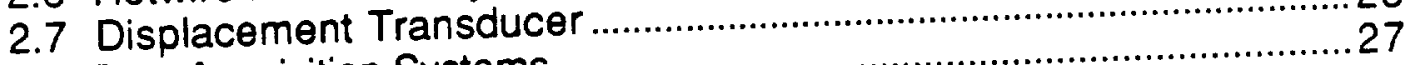

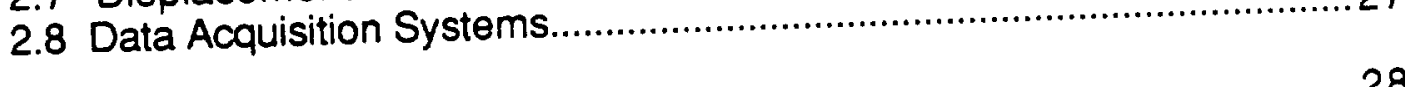

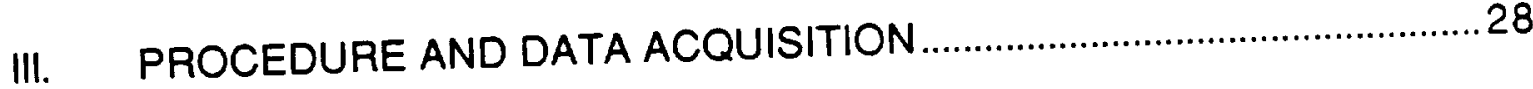

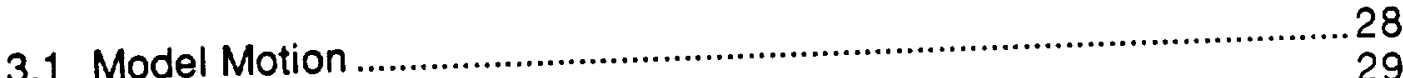

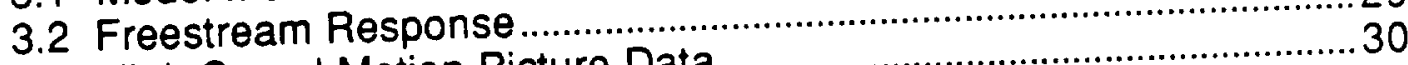

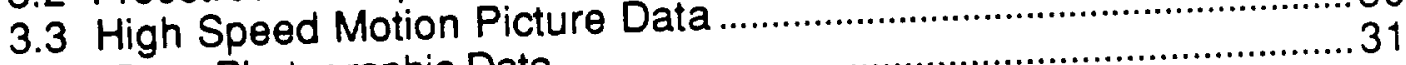

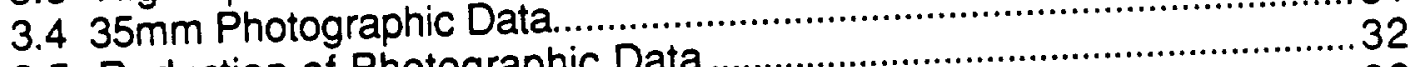

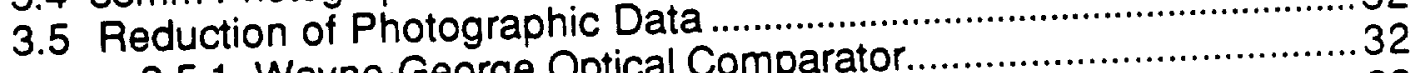

3.5.1 Wayne-George Optical Comparator............................. 33

3.5.2 Wayne-George to DEC Interface. 
3.5.3 Wayne George Accuracy.......

3.5.4 Data Acquisition with the Wayne-George .............................35

3.5.5 Accuracy of Reduced Data

IV. RESULTS

4.1 Introduction.

4.2 Model Motion.

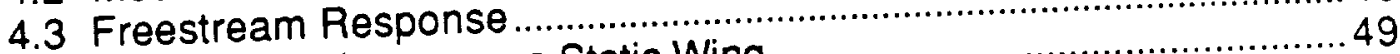

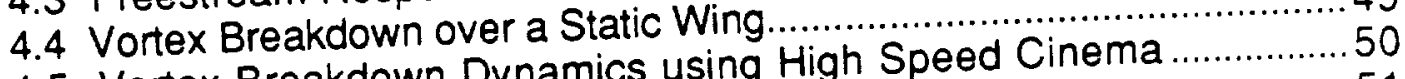

4.5 Vortex Breakdown Dynamics using High Speesonse

4.5.1 General Description of Vortex Flow Response ....................52

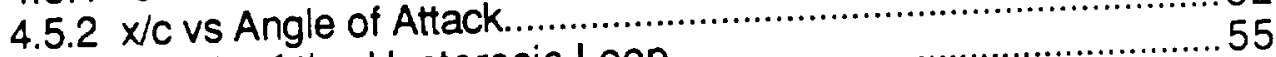

4.5.3 Width of the Hysteresis Loop..................................

4.5.4 Phase Difference between Breakdown Location and ......57

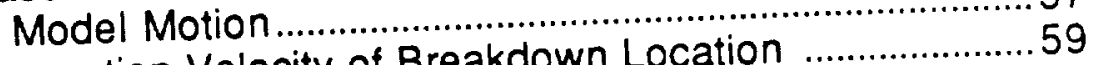

4.5.5 Propagation Velocity of Breakdown Location ..............61

4.5.6 Oscillation of Breakdown Position ......................................63

4.5.7 Vortex Core Inclination Angle................................................6. 64

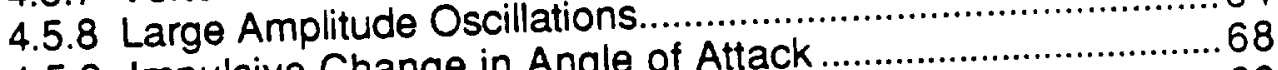

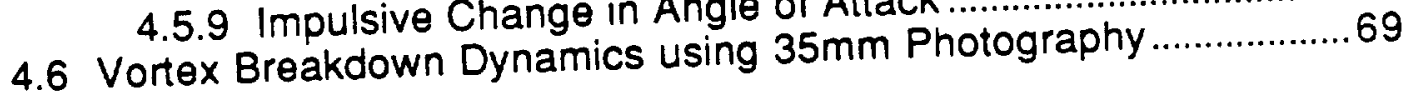

V. CONCLUSIONS AND RECOMMENDATIONS .....................................71

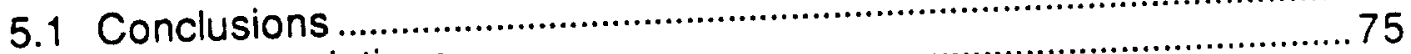

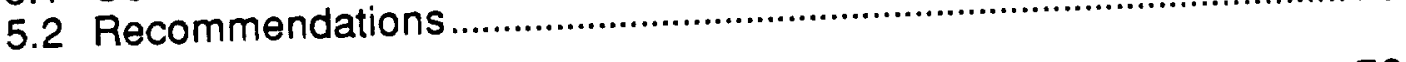

FIGURES

APPENDIX A: Discrete Fourier Transforms of Model Motion ............................133

APPENDIX B: Freestream Velocity Fluctuation and Model Position.................140

APPENDIX C: Chordwise Breakdown Location and Model Position ...............147 


\section{LIST OF TABLES}

Table

3.1 Digitized High Speed Movie Cases

40

4.1 Harmonic Distortion in the Sinusoidal Pitching Motion of the Delta Wing Model

4.2 Response of the Freesteam Flow due to the Varying Tunnel Blockage Caused by the Oscillating Model.

4.3 Cases for which the Reynolds Number was Constant and the Reduced Frequency Varied. Angle of Attack Range $=29^{\circ}$ to $39^{\circ}$

4.4 Cases for which the Reduced Frequency was Constant and the Reynolds Number Varied. Angle of Attack Range $=29^{\circ}$ to $39^{\circ}$

4.5 Average rms of Breakdown Location during Oscillatory Pitching.........62 


\section{LIST OF FIGURES}

Figure

Page

1.1 Vortex Flow over a Delta Wing

1.2 Schematic of Leading Edge Flow Patterns......................................... 79

Wind Tunnel Facility

Delta Wing Model

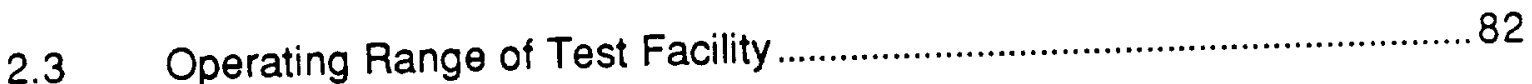

2.4 Schematic of Delta Wing Pitching Mechanism ....................................83

2.5 Schematic of Pitching Mechanism Control Box....................................84

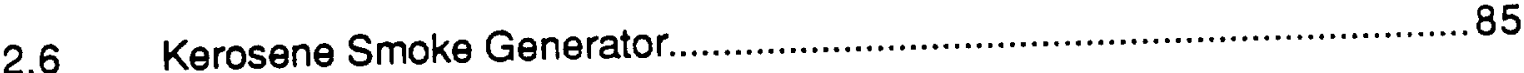

2.8 Kerosene Smoke Filaments Impinging Model Apex............................87

2.9 Titanium Tetrachloride Injection System ...........................................88

$2.10 \quad 16 \mathrm{~mm}$ Motion Picture Camera Setup................................................89

$2.1135 \mathrm{~mm}$ SLR Camera Setup...............................................................90

2.12 Linear Variable Differential Transformer Schematic............................91

3.1 Example of a Photographic Image obtained from a Side View ............92

3.2 Schematic of Photographic Image obtained from a Side View ............92

3.3 Wayne-George Optical Comparator Configuration...............................93

3.4 Example of a Photographic Image obtained from a Top View.............94

3.5 Schematic of Photographic Image obtained from a Top View..............94

3.6 Representative Plot lllustrating how Angle of Attack Varies between Pitching Cycles at Specific Points within the Cycle 95

3.7 Representative Plot Illustrating how the Chordwise Breakdown Location Varies between Pitching Cycles at Specific Points within the Cycle 
4.1 Attachment of LVDT to Delta Wing Pitching Mechanism......................97

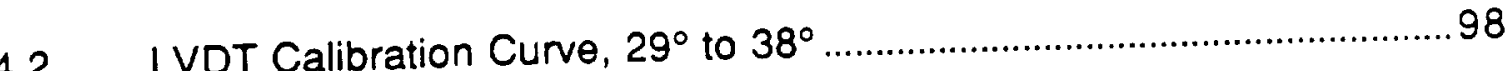

4.3a One Data Record of the Freesteam Response due to Model Motion for a Representative Case .99

4.3b Ensemble Average of 100 Data Records of the Freestream Response due to Model Motion for a Representative Case and Decreasing Angle of Attack. Angle of Attack Range $=29^{\circ}$ to $39^{\circ}, U=30 \mathrm{Ht} / \mathrm{s}, R \theta=260,000$

4.5a Discrete Fourier Transform of the Variation in Chordwise Breakdown Location from Mean Position at an Angle of Attack of $29^{\circ}$. $U=30 \mathrm{ft} / \mathrm{s}, R e=260,000$

4.5b Discrete Fourier Transform of the Variation in Chordwise Breakdown Location from Mean Position at an Angle of Attack of $39^{\circ} . \mathrm{U}=30 \mathrm{tt} / \mathrm{s}, \mathrm{Re}=260,000$.

4.6 RMS of the Chordwise Breakdown Location at Static Angles of Attack throughout the Range of Pitching Motion. Angle of Attack Range $=29^{\circ}$ to $39^{\circ}, U=30 \mathrm{H} / \mathrm{s}, R e=260,000$

4.7a-j Sequence of Photographs made from $16 \mathrm{~mm}$ Movie Film which Illustrates how the Chordwise Breakdown Location Varies throughout a Pitching Cycle. Angle of Attack Range $=29^{\circ}$ to $39^{\circ}$ 103

$4.8 a$

Chordwise Position of Vortex Breakdown during Oscillatory

Motion. Angle of Attack Range $=29^{\circ}$ to $39^{\circ}, \mathrm{k}=0.05, \mathrm{U}=30 \mathrm{ft} / \mathrm{s}$,

$R \theta=260,000$

4.8b Chordwise Position of Vortex Breakdown during Oscillatory

Motion. Angle of Attack Range $=29^{\circ}$ to $39^{\circ}, k=0.10, U=30 \mathrm{ft} / \mathrm{s}$,

$R e=260,000$

4.8c Chordwise Position of Vortex Breakdown during Oscillatory

Motion. Angle of Attack Range $=29^{\circ}$ to $39^{\circ}, \mathrm{k}=0.20, \mathrm{U}=30 \mathrm{tt} / \mathrm{s}$,

$R \theta=260,000$

4.8d Chordwise Position of Vortex Breakdown during Oscillatory

Motion. Angle of Attack Range $=29^{\circ}$ to $39^{\circ}, k=0.30, U=30 \mathrm{tt} / \mathrm{s}$,

$R e=260,000$ 
4.9a Chordwise Position of Vortex Breakdown during Oscillatory

Motion. Angle of Attack Range $=29^{\circ}$ to $39^{\circ}, \mathrm{k}=0.20, \mathrm{U}=10$ and $20 \mathrm{ft} / \mathrm{s}, \mathrm{Re}=90,000$ and 175,000

4.9b Chordwise Position of Vortex Breakdown during Oscillatory Motion. Angle of Attack Range $=29^{\circ}$ to $39^{\circ}, \mathrm{k}=0.20, \mathrm{U}=30$ and $40 \mathrm{ft} / \mathrm{s}, \mathrm{Re}=260,000$ and 350,000

4.9c Chordwise Position of Vortex Breakdown during Oscillatory Motion. Angle of Attack Range $=29^{\circ}$ to $39^{\circ}, \mathrm{k}=0.20, \mathrm{U}=10,20$, 30 , and $40 \mathrm{ft} / \mathrm{s}, \mathrm{Re}=90,000,175,000,260,000$, and 350,000

4.10a Width of the Hysteresis Loops, presented in Figures 4.8a-d, over the Angle of Attack Range of $29^{\circ}$ to $39^{\circ}$ for Reduced Frequencies of $k=0.05,0.10,0.20,0.30$. $U=30 \mathrm{ft} / \mathrm{s}, R e=260,000$

$4.10 \mathrm{~b}$ Width of the Hysteresis Loops, presented in Figures $4.9 \mathrm{a}-\mathrm{c}$, over the Angle of Attack Range of $29^{\circ}$ to $39^{\circ}$ for Reynolds Numbers of $R e=90,000,175,000,260,000$, and $350,000 . k=0.20$, $U=10,20,30$, and $40 \mathrm{ft} / \mathrm{s}$

4.11 Representative Plot of Chordwise Breakdown Location and Model Angular Position as a Function of Period.

4.12 Phase Lag of the Vortex Flow as a Function of Reduced Frequency for a Reynolds Number of 260,000 . Angle of Attack Range $=29^{\circ}$ to $39^{\circ}, \mathrm{U}=30 \mathrm{ft} / \mathrm{s}$

4.13 Phase Lag of the Vortex Flow as a Function of Reynolds Number for a Reduced Frequency of 0.20. Angle of Attack Range $=29^{\circ}$ to

4.14 Phase Lag of the Vortex Flow as a Function of Pitching Frequency for a Reynolds Number of 260,000 and a Reduced Frequency of 0.20 . Angle of Attack Range $=29^{\circ}$ to $39^{\circ}$

4.15 Breakdown Propagation Velocity relative to the Delta Wing throughout a Pitching Cycle for Reduced Frequencies of $k=0.05,0.10,0.20$, and 0.30 . Angle of Attack Range $=29^{\circ}$ to $39^{\circ}, U=30 \mathrm{tt} / \mathrm{s}, R \theta=260,000$

4.16 Breakdown Propagation Velocity relative to the Delta Wing throughout a Pitching Cycle for Reynolds Numbers of 90,000 , 175,000,260,000, and 350,000. Angle of Attack Range $=29^{\circ}$ to $39^{\circ}, \mathrm{k}=0.20, \mathrm{U}=10,20,30$, and $40 \mathrm{ft} / \mathrm{s}$. 
4.17 Reduced Breakdown Propagation Velocity relative to the Delta Wing throughout a Pitching Cycle for Reduced Frequencies of $k=0.05,0.10,0.20$, and 0.30 . Angle of Attack Range $=29^{\circ}$ to $39^{\circ}, U=30 \mathrm{tt} / \mathrm{s}, \mathrm{R \theta}=260,000$

4.18 Reduced Breakdown Propagation Velocity relative to the Delta Wing throughout a Pitching Cycle for Reynolds Numbers of $90,000,175,000,260,000$, and 350,000 . Angle of Attack Range $=29^{\circ}$ to $39^{\circ}, k=0.20, U=10,20,30$, and $40 \mathrm{ft} / \mathrm{s}$

4.19a RMS of the Chordwise Breakdown Location at Various Angles of Attack throughout a Pitching Cycle. Angle of Attack Range $=29^{\circ}$ to $39^{\circ}, k=0.20, U=30 \mathrm{ft} / \mathrm{s}, \mathrm{Re}=260,000$

4.19b RMS of the Chordwise Breakdown Location at Various Angles of Attack throughout a Pitching Cycle. Angle of Attack Range $=29^{\circ}$ to $39^{\circ}, \mathrm{k}=0.20, \mathrm{U}=20 \mathrm{ft} / \mathrm{s}, \mathrm{Re}=175,000$

4.20a Photograph used to Demonstrate that the Vortex Core can be Assumed to be Straight. Angle of Attack 29,$U=30 \mathrm{ft} / \mathrm{s}$, $R e=260,000$

4.20b Photograph used to Demonstrate that the Vortex Core can be Assumed to be Straight. Angle of Attack $39^{\circ}, U=30 \mathrm{ft} / \mathrm{s}$,

$R \theta=260,000$

4.21 Vortex Core Inclination Angle during Oscillatory Motion. Angle of Attack Range $=29^{\circ}$ to $39^{\circ}, \mathrm{K}=0.05$ and $0.10, \mathrm{U}=30 \mathrm{ft} / \mathrm{s}$, $R e=260,000$

4.22 Vortex Core Inclination Angle during Oscillatory Motion. Angle of

Attack Range $=29^{\circ}$ to $39^{\circ}, \mathrm{k}=0.20$ and $0.30, \mathrm{U}=30 \mathrm{ft} / \mathrm{s}$,

$R e=260,000$

4.23 Vortex Core Inclination Angle during Oscillatory Motion. Angle of Attack Range $=29^{\circ}$ to $39^{\circ}, \mathrm{K}=0.20, \mathrm{U}=10$ and $20 \mathrm{ft} / \mathrm{s}$, $R e=90,000$ and 175,000

4.24 Vortex Core Inclination Angle during Oscillatory Motion. Angle of Attack Range $=29^{\circ}$ to $39^{\circ}, \mathrm{K}=0.20, \mathrm{U}=30$ and $40 \mathrm{ft} / \mathrm{s}$, $R e=260,000$ and 350,000

4.25a-j Sequence of Photographs made from $16 \mathrm{~mm}$ Movie Film which Illustrates how the Chordwise Breakdown Location Varies throughout a Pitching Cycle. Angle of Attack Range $=0^{\circ}$ to $45^{\circ}$, $k=0.30, U=30 \mathrm{fts}, \mathrm{R} \theta=260,000$ 
4.26a-j Sequence of Photographs made from $16 \mathrm{~mm}$ Movie Film which Illustrates the Separation and Reattachment Processes of the Vortex Flow.

4.27a Chordwise Position of Vortex Breakdown during Oscillatory Motion. Angle of Attack Range $=0^{\circ}$ to $45^{\circ}, k=0.05$ and 0.30 , $\mathrm{U}=30 \mathrm{ft} / \mathrm{s}, \mathrm{Re}=260,000$

4.27b Chordwise Position of Vortex Breakdown during Oscillatory Motion for a Large and Small Amplitude Case, and Static Data. Angle of Attack Range $=0^{\circ}$ to $45^{\circ}$ and $29^{\circ}$ to $39^{\circ}, \mathrm{k}=0.30$, $\mathrm{U}=30 \mathrm{ft} / \mathrm{s}, \mathrm{Re}=260,000$

4.28 Vortex Core Inclination Angle during Oscillatory Motion. Angle of Attack Range $=0^{\circ}$ to $45^{\circ}, \mathrm{k}=0.05$ and $0.30, \mathrm{U}=30 \mathrm{ft} / \mathrm{s}$, $\operatorname{Re}=260,000$

4.29a Response of the Chordwise Breakdown Location due to an Impulsive Pitch-up in Angle of Attack. Angle of Attack Range $=28^{\circ}$ to $37^{\circ}, \mathrm{k}=0.45, \mathrm{U}=30 \mathrm{ft} / \mathrm{s}, \mathrm{Re}=260,000$

4.29b Response of the Chordwise Breakdown Location due to an Impulsive Pitch-down in Angle of Attack. Angle of Attack Range $=28^{\circ}$ to $37^{\circ}, k=0.45, U=30 \mathrm{t} / \mathrm{s}, R e=260,000$

4.30 Spanwise Position of Vortex Breakdown during Oscillatory Motion. Angle of Attack Range $=29^{\circ}$ to $39^{\circ}, k=0.05,0.20$ and $0.30, \mathrm{U}=30 \mathrm{ft} / \mathrm{s}, \mathrm{Re}=260,000$

A1 DFT of Model Motion. Angle of Attack Range $=29^{\circ}$ to $39^{\circ}$, $\mathrm{k}=0.05, \mathrm{U}=30 \mathrm{ft} / \mathrm{s}$

A2 DFT of Model Motion. Angle of Attack Range $=29^{\circ}$ to $39^{\circ}$, $k=0.30, U=30 \mathrm{ft} / \mathrm{s}$

A3 DFT of Model Motion. Angle of Attack Range $=29^{\circ}$ to $39^{\circ}$, $k=0.20, U=10 \mathrm{t} / \mathrm{s}$

A4 DFT of Model Motion. Angle of Attack Range $=29^{\circ}$ to $39^{\circ}$, $k=0.20, U=40 \mathrm{ft} / \mathrm{s}$

A5 DFT of Model Motion. Angle of Attack Range $=0^{\circ}$ to $45^{\circ}$, $\mathrm{k}=0.05, \mathrm{U}=30 \mathrm{ft} / \mathrm{s}$

A6 DFT of Model Motion. Angle of Attack Range $=0^{\circ}$ to $45^{\circ}$, $k=0.30, U=30 \mathrm{ft} / \mathrm{s}$

B1 Freestream Velocity Fluctuation and Model Position. Angle of Attack Range $=29^{\circ}$ to $39^{\circ}, k=0.05, U=30 \mathrm{ft} / \mathrm{s}$. 
B2 Freestream Velocity Fluctuation and Model Position. Angle of Attack Range $=29^{\circ}$ to $39^{\circ}, \mathrm{K}=0.05, \mathrm{U}=30 \mathrm{ft} / \mathrm{s}$.

B3 Freestream Velocity Fluctuation and Model Position. Angle of Attack Range $=29^{\circ}$ to $39^{\circ}, \mathrm{k}=0.20, \mathrm{U}=10 \mathrm{ft} / \mathrm{s}$

B4 Freestream Velocity Fluctuation and Model Position. Angle of Attack Range $=29^{\circ}$ to $39^{\circ}, \mathrm{k}=0.20, \mathrm{U}=40 \mathrm{ft} / \mathrm{s}$

B5 Freestream Velocity Fluctuation and Model Position. Angle of Attack Range $=0^{\circ}$ to $45^{\circ}, \mathrm{k}=0.05, \mathrm{U}=30 \mathrm{ft} / \mathrm{s}$

B6 Freestream Velocity Fluctuation and Model Position. Angle of Attack Range $=0^{\circ}$ to $45^{\circ}, \mathrm{k}=0.30, \mathrm{U}=30 \mathrm{ft} / \mathrm{s}$

C1 Chordwise Breakdown Location and Model Position. Angle of Attack Range $=29^{\circ}$ to $39^{\circ}, \mathrm{k}=0.05, \mathrm{U}=30 \mathrm{tt} / \mathrm{s}, \mathrm{Re}=260,000$.

C2 Chordwise Breakdown Location and Model Position. Angle of Attack Range $=29^{\circ}$ to $39^{\circ}, \mathrm{k}=0.10, \mathrm{U}=30 \mathrm{ft} / \mathrm{s}, \mathrm{Re}=260,000$

C3 Chordwise Breakdown Location and Model Position. Angle of Attack Range $=29^{\circ}$ to $39^{\circ}, \mathrm{k}=0.20, \mathrm{U}=30 \mathrm{tt} / \mathrm{s}, \mathrm{Re}=260,000$.

C4 Chordwise Breakdown Location and Model Position. Angle of Attack Range $=29^{\circ}$ to $39^{\circ}, \mathrm{K}=0.30, \mathrm{U}=30 \mathrm{ft} / \mathrm{s}, \mathrm{Re}=260,000$

C5 Chordwise Breakdown Location and Model Position. Angle of Attack Range $=29^{\circ}$ to $39^{\circ}, k=0.20, U=10 \mathrm{ft} / \mathrm{s}, R e=90,000$

C6 Chordwise Breakdown Location and Model Position. Angle of Attack Range $=29^{\circ}$ to $39^{\circ}, k=0.20, U=20 \mathrm{tt} / \mathrm{s}, R_{\theta}=175,000$

C7 Chordwise Breakdown Location and Model Position. Angle of Attack Range $=29^{\circ}$ to $39^{\circ}, \mathrm{k}=0.20, \mathrm{U}=30 \mathrm{tt} / \mathrm{s}, \mathrm{Re}=260,000$

C8 Chordwise Breakdown Location and Model Position. Angle of Attack Range $=29^{\circ}$ to $39^{\circ}, \mathrm{k}=0.20, \mathrm{U}=40 \mathrm{ft} / \mathrm{s}, \mathrm{Re}=350,000$

C9 Chordwise Breakdown Location and Model Position. Angle of Attack Range $=0^{\circ}$ to $45^{\circ}, \mathrm{k}=0.05, \mathrm{U}=30 \mathrm{ft} / \mathrm{s}, \mathrm{Re}=260,000$

C10 Chordwise Breakdown Location and Model Position. Angle of Attack Range $=0^{\circ}$ to $45^{\circ}, \mathrm{k}=0.30, \mathrm{U}=30 \mathrm{tt} / \mathrm{s}, \mathrm{Re}=260,000$ 


\section{NOMENCLATURE}

\section{Symbols}

\begin{tabular}{|c|c|}
\hline c & Root chord length \\
\hline$f$ & Pitching frequency \\
\hline$g$ & Gravitational constant \\
\hline k & Reduced frequency, $\frac{2 \pi f c}{u}$ \\
\hline K & Nondimensional pitch rate, $\frac{\dot{\alpha} \tau}{2}$ \\
\hline $\operatorname{Re}$ & Reynolds number, $\frac{\mathrm{uc}}{\mathrm{v}}$ \\
\hline $\mathbf{s}$ & Local semi-span \\
\hline$t$ & Time (seconds) \\
\hline $\mathrm{T}$ & Dimensionless time, $\frac{t}{\tau}$ \\
\hline$u$ & Freestream Velocity \\
\hline U & Freestream Velocity \\
\hline$x$ & Distance from apex parallel with wing chord \\
\hline$y$ & Distance from root chord parallel to trailing edge \\
\hline$\alpha$ & Angle of Attack (degrees) \\
\hline$\dot{\alpha}$ & Pitch rate (radians/second) \\
\hline$\beta$ & Vortex core inclination angle (degrees) \\
\hline$\mu$ & Reduced Velocity, $\frac{u}{c f}$ \\
\hline$\tau$ & Convective time unit, $\frac{\mathrm{c}}{\mathrm{u}}$ (seconds) \\
\hline$v$ & Kinematic viscosity of air \\
\hline$\omega$ & Angular Velocity \\
\hline
\end{tabular}


Abbreviations

LVDT Linear Variable Differential Transformer

DFT Discrete Fourier Transform

rms Root mean square

SCR Silicon Controlled Rectifier

SLR Single Lens Reflex

$\mathrm{TiCl}_{4}$ Titanium tetrachloride 


\section{ACKNOWLEDGEMENTS}

The author would like to acknowledge and thank the following persons for their help, advice, and contributions:

Dr. Stephen M. Batill and Dr. Robert C. Nelson, faculty advisors, for their guidance and recommendations which have been instrumental in this research.

Roger Davis, for the time he spent machining and fabricating the unsteady pitching mechanism and for his advice in the design.

Joe Hollkamp, Mike Brendel, and Thom Bradley, for their advice in the data collection and reduction, and for their help with the Aero Lab computing systems in general.

Dr. $\mathrm{Ng}$ and Dr. Gad-el-Hak, for their suggestions made during the writing of this thesis.

Mike Swadner, for the construction of experimental equipment and the advice he offered, and to Roger Kenna for his help with development of photographic data.

The work was sponsored by the NASA Langley Research Center NAG-1-727, and the University of Notre Dame. 


\section{CHAPTER I}

INTRODUCTION

\subsection{Perspective}

Over the past few decades, the flow over a steady delta wing has been studied extensively. At low angles of attack, the flow is attached to the top and bottom surfaces of the wing, and lift is produced in the same manner as on a conventional airfoil. At moderate angles of attack, the flow separates from the leading edge of the wing to form two counter- rotating vortices. It is these separated vortices, rather than attached flow, which are responsible for producing lift. The lift increases non-linearly with angle of attack to a maximum value, and then tapers off slowly. At the higher angles of attack, a phenomenon known as vortex breakdown occurs, in which the vortex flow undergoes a sudden transformation. This breakdown appears first in the wake, and then with increasing angle of attack the breakdown location moves upstream and eventually above the top surface of the wing. If vortex breakdown occurs over the top surface of wing, the large suction pressures which are associated with the leading edge vortices are reduced. This results in a loss of lift and a change in the pitching moment.

Most of our modern military aircrafts (which possess delta wings), go through many dynamic motions from takeoff through landing. These dynamic motions occur especially in dogfight situations, where an aircraft is 
maneuvering and changing speeds almost constantly. Future aircraft may be required to operate at angles of attack beyond static stall in order to increase their combat effectiveness (Herbst, 1983). Therefore, to improve aircraft performance, it is important that we gain an understanding of the complex flow patterns which occur over a delta wing during these dynamic flight maneuvers.

The unsteady aerodynamic characteristics resulting from a change in angle of attack, for example, can be significantly different from those of the static case. Under oscillatory or transient motion, the vortices which are created by leading edge separation change strength and position as a function of timevarying angle of attack. Therefore, the location of vortex breakdown will likewise become time dependent. These changes in the vortex flow do not take place immediately with the dynamic motion, due to the convective time lag of the adjusting flow field (Malcolm, 1981).

The different types of research which have been conducted on the complex unsteady aerodynamic properties associated with dynamic vortex flows is presented in the next section. However, additional research is needed to improve our understanding of the phenomena. Through the use of smoke flow visualization, this investigation examines the response of the vortex flow and breakdown position on a $70^{\circ}$ delta wing undergoing oscillatory sinusoidal pitching motions, and impulsive changes in angle of attack. This research contributes to the understanding of the vortex flow dynamic response, and hopefully provides practical information for the development of high performance aircraft. 


\subsection{Background Study}

As mentioned previously, there has been much work performed on the vortex flow over a steady delta wing. On the other hand, little work has been conducted on the response of the vortex flow due to dynamic motion. In the following sections, some findings from other investigations are discussed which include experiments performed on: a pitching delta wing, a plunging delta wing, the effect of unsteady freestream flow, and hysteresis associated with static changes in angle of attack (static hysteresis). Before the unsteady cases are presented, however, a brief explanation of the steady vortex flow will be given.

\subsubsection{The Leading Edge Vortex and Vortex Breakdown}

At low angles of attack, the flow over a delta wing is attached to both the upper and lower surfaces, and lift is produced as on a conventional airfoil. At moderate angles of attack, two counter-rotating vortices form over the upper surface of the delta wing as shown in Figure 1.1. As the flow approaches the wing, it attaches to the lower surface and moves towards the leading edge. Upon reaching the leading edge, the flow separates and forms a vortex sheet because it is unable to negotiate the sharp turn. A spanwise pressure gradient on the upper surface causes the free shear layer to move inward and roll up to form a tightly bound spiraling vortex. As a result of this vortex, a suction peak is produced on the upper surface of the wing at a spanwise location which coincides with that of the vortex.

The surface flow on the upper surface is directed outward due to the vortex, and because of the large pressure gradient which exists between the 
suction peak and the leading edge, the flow separates to form a small secondary vortex (Erickson, 1981). This secondary vortex tends to push the primary vortex upwards and inwards. The flow then reattaches and continues to move towards the leading edge. At the leading edge, the flow then becomes entrained into the free shear layer. A schematic of the vortex and surface flow is shown in Figure 1.2.

As the angle of attack increases, the vortex strength increases. Core velocities can reach three times that of the freestream flow. As the angle of attack continues to increase, a change takes place in the vortex flow which results in a deceleration of the vortex core axial flow, a decrease in the tangential velocity, and an increase in the vortex size. This phenomenon is called vortex breakdown. On a static wing, the position at which breakdown occurs along the vortex depends primarily on the leading edge sweep angle, and angle of attack (Elle, 1958). The breakdown first occurs in the wake, downstream of the trailing edge. As the angle of attack increases, the breakdown position moves forward to a location above the surface of the wing. This results in a loss of lift and a change in pitching moment.

\subsubsection{Unsteady Vortex Dynamics}

As the delta wing is pitched, plunged, or taken through some other type of unsteady motion, there is a time lag in the response of the vortex flow which can result in temporarily delayed vortex separation at low angles of attack, or temporarily delayed vortex breakdown at higher angles of attack. By taking advantage of these unsteady effects, a high performance aircraft might be able to perform certain aerobatic maneuvers more quickly and efficiently. For delta wings undergoing cyclic motions, a hysteresis develops in the vortex flow 
relative to the static case which increases with the frequency or rate of the motion. Other changes also occur in the vortex flow which are not readily apparent in the steady case. In the following, some specific findings are presented from research which has been previously performed on unsteady vortex dynamics. Most of the data were obtained from observations made through the use of flow visualization.

\section{The Effect of Pitching}

When investigating the response of the vortex flow on a delta wing due to cyclic motion, a reduced frequency $(k)$ is typically defined as $k=\omega c / u$ where $\omega$ is the angular velocity, $c$ the root chord, and $u$ the freesteam velocity. However, several variations of this definition are used by other investigators. In this study, the definition used is; $k=2 \pi f \mathrm{c} / \mathrm{u}$, where $f$ is the frequency of oscillation. To aid in comparison, all mentions of reduced frequency pertaining to other investigations have been converted to the definition used in this study.

Gad-el Hak and Ho (1985) sinusoidally pitched a $45^{\circ}$ sweep delta wing about the quarter-chord position from $0^{\circ}$ to $45^{\circ}$ at reduced frequencies ranging from 0.10 to 6.0 . On the upstroke, flow separation started at the trailing edge and propagated towards the apex. The propagation velocity of vortex flow separation along the leading edge was approximately equal to that of the freestream velocity. On the downstroke, they observed no propagation phenomenon along the leading edge, rather the vortex flow reattached along the entire leading edge at the same time.

Gad-el-Hak and Ho (1985) also found the existence of a hysteresis in the vortex flow on a $45^{\circ}$ sweep angle wing which was pitched between $10^{\circ}$ and $20^{\circ}$ in sinusoidal motion for chord Reynolds numbers of 25,000 to 350,000 . The 
flow patterns at any particular angle of attack were very different on the upstroke and downstroke. The hysteresis was quantified from the growth and decay of the leading edge vortex as the wing oscillated for reduced frequencies of $k=$ $0.50,1.0$ and 2.0. The size of the vortex, determined by the height of a dye marker above the wing at a specific $x / c$, was presented as a function of instantaneous angle of attack. At angles of attack of less than $15^{\circ}$, the hysteresis was not a function of reduced frequency. In this range of angle of attack, the amount of hysteresis was the same for each reduced frequency. Also, the size of the vortex in the static case was approximately equal to the averaged values of vortex size on the upstroke and downstroke. At a reduced frequency of $k=0.50$, the average value of the vortex size (upstroke and downstroke) followed the static case fairly well throughout the entire pitching cycle. A deviation from this trend occurred as the reduced frequency was increased, which was more evident at the higher angles of attack. This deviation was due to the inability of the vortices to reach their fully developed static size on the upstroke before the wing changed to downstroke. A quasisteady state was not attained in the vortex flow, with a hysteresis observed at a reduced frequency as low as $k=0.10$.

Rockwell et al (1987) also reported the existence of a substantial hysteresis in the vortex flow relative to the static case. A $45^{\circ}$ sweep delta wing was pitched about its trailing edge sinusoidally from $5^{\circ}$ to $20^{\circ}$ at reduced frequencies of $k=0.16$ to 10.68 . The chord Reynolds number was varied between 5,800 and 45,000 . A hysteresis in the vortex flow became evident when the chordwise position of vortex breakdown was quantified as a function of instantaneous angle of attack. They found substantial hysteresis relative to the static case at a reduced frequency as low as $k=0.16$. The trend of the hysteresis was generally the same for higher values of reduced frequency until 
$k=1.75$ was attained. At $k=1.75$ the hysteresis effect started to reverse, and at reduced frequencies of $k=4.77$ and higher, the sense of the hysteresis was opposite to that of the $k<1.75$ cases.

Bragg and Saltani (1987) obtained 6-component balance data on a $70^{\circ}$ delta wing sinusoidally oscillating between $0^{\circ}$ and $55^{\circ}$ at reduced frequencies of $k=0$ to 0.112 . Preliminary unpublished data showed a strong influence of pitch rate on the unsteady forces and moments. Hysteresis loops were seen in all oscillating model data.

Gad-el Hak and Ho (1985) found that the effect of Reynolds number on vortex flow was small in the range of $R \theta=25,000$ to 340,000 . Two tests were conducted at Reynolds numbers of 25,000 and 340,000 at a reduced frequency of $k=2.0$ for an angle of attack range was $0^{\circ}$ to $30^{\circ}$. No measurable difference in the size of the vortices was observed between the two cases. This indicated that changes in the vortex flow were due mainly to inertial effects and not to viscosity.

Gilliam et al (1987), pitched a delta wing at a constant pitch rate from $0^{\circ}$ to $60^{\circ}$. The pitching rate primarily affected the length of time the vortex remained over the wing surface. The duration time decreased with increasing pitch rate, however the vortex remained over the wing until higher angles of attack. Also, the vortex remained more coherent and its diameter increased with increasing pitch rate. No delay was detected in the response of the flow upon initiation of the pitching motion.

A similar experiment was performed by Reynolds and Abtahi (1987) in which a delta wing of aspect ratio one was pitched at a constant rate of $K=0.06$. The wing was pitched from $30^{\circ}$ to $51^{\circ}$, and from $51^{\circ}$ to $30^{\circ}$ at 4 root chord Reynolds numbers between 19,000 and 65,000 . Large time lags associated with the location of vortex breakdown relative to the static case were observed, 
and a hysteresis was detected in the response of the vortex flow between the pitch-up and pitch-down cases. Another series of tests were conducted in which the wing was pitched down from an angle of attack of $51^{\circ}$ to angles of attack varying from $45^{\circ}$ to $20^{\circ}$. For these motions, the response times required for the breakdown location to reach a steady state were found to range 1 to 30 convective time units after the motion was completed. A convective time is the time required for the flow to travel one chord length.

Gad-el-Hak et al (1983) observed near the leading edge, a rolling up of the shear layer which formed discrete vortices along approximately straight lines emanating from the apex for a static model. The discrete vortices were also observed in the unsteady case when the model was sinusoidally oscillated. However in this case the discrete vortices were altered and modulated by the unsteady motion which had an order of magnitude lower frequency. Rockwell et al (1987) were able to attain active control of the leading edge vortices for both large and small amplitude pitching motions by proper selection of an excitation frequency based on the fundamental frequency of the shear layer separation as observed by Gad-el-Hak and Blackwelder (1986).

\section{The Effect of Plunging}

A plunging delta wing is one which goes through a vertical displacement without physically changing angle of attack. However, this motion results in an effective change in angle of attack due to the vertical velocity of the model relative to the freesteam flow. A plunging model can be used to simulate an aircraft flying into a gust for example.

Lambourne et al, (1969) investigated the transient behavior of the leading edge vortices over a delta wing due to a sudden change of incidence 
by applying a constant velocity plunging motion for a limited time. Preliminary experiments showed that a new steady state condition was reached by the flow in the time it took for the freestream flow to move one chord length for a zero to positive incidence plunging motion. The height of the vortex core was measured at $x / c$ locations of $1 / 2,2 / 3$, and 1.0. Over the course of the plunging motion, the vortex core first appeared close to the leading edge and then, with increasing height, moved inward over the wing. At the end of the plunge the vortex height did not reduce immediately, and the vortex flow took longer to reach a steady state at the trailing edge than it did at the other $x / c$ locations of $1 / 2$ and $2 / 3$. At the start of the plunging motion, no similar delay was observed. In another experiment, the wing was plunged starting at an incidence of $11^{\circ}$ to a higher effective incidence. At an incidence of $11^{\circ}$, a steady vortex existed above the wing. Upon initiation of the plunging motion, a second vortex was generated at the leading edge. The newly generated vortex moved to a position higher than that of the original vortex, and the original vortex moved under the secondary vortex and was consumed.

Malty et al (1963) performed a similar test, except that the delta wing was sinusoidally oscillated in heave. The wing was oscillated at a reduced frequencies of $k=1.13$ and 3.40 , at root chord Reynolds numbers of $2 \times 10^{6}$ and $6 \times 10^{6}$ respectively. The model had an aspect ratio of 1.0 and the amplitude of the heaving motion was $1 / 24$ the root chord. This corresponded to a change in angle of attack of $\pm 1.35^{\circ}$ at the lower reduced frequency and $\pm 4.05^{\circ}$ at the higher reduced frequency. The initial incidence of the wing was $22^{\circ}$. A phase lag was found between the height of the vortex core and the oscillatory heaving motion of the model. At the reduced frequency of 1.13 the phase lag was $51^{\circ}$, and at the reduced frequency of 3.40 the phase lag was $60^{\circ}$. 
Lee et al (1987) investigated the response of a delta wing of aspect ratio 2 under periodic acceleration and deceleration of the freesteam velocity in a water tunnel. The velocity was varied by approximately $25 \%$ of the mean value in a triangular pattern. Simultaneous measurements of the aerodynamic forces and visualization of the flow were performed. The greatest pressure gradient which was experienced by the model was equivalent to it being subjected to a $10 \mathrm{~g}$ acceleration or deceleration in air. During the acceleration part of the cycle the vortex broke down earlier and in the deceleration phase the breakdown was delayed. This variation was less than $10 \%$ of the root chord. Lambourne and Bryer (1961) observed the same phenomenon when they exposed a flat plate delta wing to accelerations and decelerations. Lee et al (1987) found that the vortex appeared to spiral in a tighter fashion during acceleration and uncoil during deceleration. Force balance data indicated that the phase-averaged lift coefficients were lower than the static lift coefficients in the deceleration phase and higher in the accelerating phase, indicating a strong hysteresis in the aerodynamic forces. The variation in lift was attributed to the inertia of the unsteady fluid which increased with decreasing oscillation period.

\section{Static Hysteresis}

Several researchers have reported a hysteresis associated with unsteady vortex flow. The question arises as to whether a hysteresis exist in the static case, i.e., are the flow characteristics the same for both static increases and static decreases in angle of attack. Cunningham (1985) investigated the static hysteresis associated with various vortex flow transition points. The 
transition points consisted of the angle of attack at which the position of vortex breakdown crosses the trailing edge of the wing, and the angle of attack at which transition to or from totally separated flow takes place. The models used were simple flat plate delta wings with rounded leading edges and sweep angles of $55^{\circ}$ to $80^{\circ}$. To test for hysteresis, the angle of attack was lowered far below the transition point of interest and then slowly increased until the transition occurred. The opposite was done for decreasing angle of attack. Little or no hysteresis was observed in the angle of attack at which the position of vortex breakdown crossed the trailing edge, and a hysteresis of approximately $3^{\circ}$ to $4^{\circ}$ was observed in the transition to and from totally separated flow.

Lowson (1963) reported a substantial hysteresis in the angle of attack at which vortex breakdown crosses the trailing edge on a $80^{\circ}$ flat plate delta wing. The work was performed in a water tunnel at a freestream velocity of $0.35 \mathrm{ft} / \mathrm{s}$ $(0.107 \mathrm{~m} / \mathrm{s})$. The angle of attack was slowly increased to $41^{\circ}$ at which point the breakdown location moved from the wake to a position above wing. When the angle of attack was reduced, the breakdown location remained over the wing until an angle of attack of $34^{\circ}$ was reached, at which point the breakdown recrossed the trailing edge.

\subsubsection{Brief Summary of Unsteady Work Reviewed}

As a delta wing is pitched or plunged in an sinusoidal fashion, a hysteresis becomes evident in the response of the vortex flow. A hysteresis has been detected at reduced frequencies as low as $k=0.10$. The hysteresis can be quantified in many ways. In previous studies, researchers have reported a 
hysteresis in the height of the vortex core above the wing at a specific $x / C$ location, the location of vortex breakdown, and in force and moment data.

A similar hysteresis was also evident when the wing was impulsively pitched or plunged from one angle of attack to another. Typically the flow responds almost instantly to the dynamic motion; but upon completion of the motion, some time is required for the flow to reach a steady state. A hysteresis has also been reported in the response of vortex flow due to accelerations and decelerations of the freesteam flow.

Lastly, a hysteresis has also been reported in static cases. This was made evident by the examination of the angle of attack at which the location of vortex breakdown crosses the trailing edge, or the angle of attack at which the flow transitions to totally separated flow. The angles of attack at which these transitions take place can be several degrees different for increasing and decreasing static angles of attack.

\subsection{Scope of Work}

The present study investigates the response of the vortex flow and breakdown location on a sinusoidally oscillating, $70^{\circ}$ flat plate delta wing with sharp leading edges, which is pitched about the $1 / 2$ chord position. The effects of reduced frequency and Reynolds number are analyzed. The reduced frequency is varied from $k=0.05$ to 0.30 at a root chord Reynolds number of 260,000 , and the Reynolds number is varied between 90,000 and 350,000 at a reduced frequency of $k=0.20$ The relatively low range of reduced frequency was selected because it is representative of the reduced frequencies experienced by the main wing on current tactical aircraft. 
Two angle of attack ranges of $29^{\circ}$ to $39^{\circ}$ and $0^{\circ}$ to $45^{\circ}$ were selected for testing. The smaller angle of attack range of $29^{\circ}$ to $39^{\circ}$ was chosen because vortex breakdown always occurs above the upper surface of the wing. Over this range of angle of attack, the breakdown location varied from approximately $0.4 \times / \mathrm{c}$ to $0.9 \times / \mathrm{c}$. The larger amplitude motion of $0^{\circ}$ to $45^{\circ}$ provided information on the location of vortex breakdown at higher angles of attack, as well as information relating to separation and reattachment of the vortex flow. Statistical information on the oscillation of vortex breakdown position was also obtained.

Also investigated was the response of the vortex flow due to an impulsive change in angle of attack. The wing was impulsively pitched-up from $28^{\circ}$ to $37^{\circ}$, and impulsively pitched-down from $37^{\circ}$ to $28^{\circ}$ degrees at a root chord Reynolds number of 260,000 . The time it took for the vortex flow to reach a steady state upon completion of the motion was determined.

In addition to acquiring aerodynamic data, a test facility and data acquisition system which could collect and reduce vortex flow information was developed. An unsteady vortex facility was built which could sinusoidally pitch a delta wing model throughout a wide range of angles of attack and reduced frequencies, as well as impulsively pitch a delta wing model. A non-intrusive method of flow visualization was also developed which provided quality flow visualization of the vortex flow about a pitching delta wing, and a method of data reduction was developed to ensemble average digitized photographic data.

This research provides a detailed analysis of the dynamic response of vortex breakdown due to unsteady motion. The information obtained increases our understanding of the complex unsteady aerodynamic flows. By obtaining a better understanding of these unsteady flow phenomena, future aircraft can be 
designed to operate more efficiently and effectively during high angle of attack dynamic maneuvers. 


\section{CHAPTER II}

\section{EXPERIMENTAL APPARATUS}

\subsection{Wind Tunnel}

The wind tunnel used in this study was one of two identical low turbulence, subsonic wind tunnels located in the University of Notre Dame Aerospace Laboratory. The tunnel is an indraft, open circuit design which exhausts to the atmosphere and is illustrated in Figure 2.1. This design allows for the removal of visualization tracers which would contaminate the flow in a closed circuit design. The flow in the tunnel is affected by atmospheric disturbances (wind gusts), and therefore relatively calm conditions should exist for tests to be conducted. To reduce the affect of atmospheric disturbances, a flow restrictor consisting of a square box filled with "honey combed" tubes was placed downstream of the test section. The large pressure drop across the flow restrictor lessened the affect of outside disturbances on flow in the test section.

The inlet of the tunnel consists of a 24:1 area contraction cone with 12 anti-turbulence screens located just upstream of the inlet. The inlet configuration provides a near uniform freestream velocity profile in the test section with a turbulence intensity of less than $0.1 \%$. The test section is $6 \mathrm{ft}$ $(183 \mathrm{~cm})$ in length with a $2 \mathrm{ft} \times 2 \mathrm{ft}(61 \mathrm{~cm} \times 61 \mathrm{~cm})$ cross section. The test section was constructed with large plate glass windows on the top and one side to provide a means of viewing the smoke tracer particles used in the flow 
visualization studies. Downstream of the test section the flow is expanded in a $13.78 \mathrm{ft}(420 \mathrm{~cm})$ diffuser with a $4.2^{\circ}$ included angle divergence. The tunnel is powered by an eight-bladed $3.94 \mathrm{ft}(120 \mathrm{~cm})$ diameter fan directly coupled to a 18.6 kW AC induction motor located at the end of the diffuser section.

\subsection{Model}

A flat plate delta wing model was used for all tests in this study and is shown in Figure 2.2. The model is $1 / 2$ in $(127 \mathrm{~mm})$ thick with a leading edge sweep of 70 degrees and a trailing edge span of 12 in $(305 \mathrm{~mm})$. The root chord is 16.48 in $(419 \mathrm{~mm})$. The leading edge is bevelled symmetrically about the top and bottom surfaces of the wing at an inclination angle of $23^{\circ}$ from the plane of the model. Smoke used for flow visualization was introduced into the flow through one of two ports located approximately one inch $(254 \mathrm{~mm})$ from the model apex and at the midline of the upper surface bevel. This was the optimal location for good quality smoke visualization determined from tests which are discussed in a later section. The ports were connected to a channel milled out from the underneath surface of the model which housed the smoke injection tubing. The model was sting mounted and was free to pitch about the $1 / 2$ root chord position. A prototype model was constructed of Plexiglas, but after being exposed to the heat produced by the 1000 Watt flood lamp used for flow visualization combined with the dynamic loadings, it had a tendency to warp in the chordwise direction. To solve this problem, an identical model was constructed of aluminum. This model was painted flat black to provide contrast for the white smoke used in flow visualization. 


\subsection{Unsteady Pitching Mechanism}

A drive system was designed and fabricated to sinusoidally pitch a delta wing model with less than $2.5 \%$ harmonic distortion over the angle of attack range of $29^{\circ}$ to $39^{\circ}$, and less than $5.0 \%$ harmonic distortion over the angle of attack range of $0^{\circ}$ to $45^{\circ}$. The system is capable of oscillating a model throughout a wide range of reduced frequencies and amplitudes about a specified mean angle of attack. A diagram showing the range of operation of the mechanism is shown in Figure 2.3. The region within the rectangle shows the actual range for which the tests in this study took place. The drive mechanism can also be used to impulsively change the angle of attack.

A schematic of the pitching mechanism is shown in Figure 2.4, as it is mounted underneath the working section. The system is powered by a $1 \mathrm{hp}$ Dayton 90 volt DC electric motor Model 2M170C with a Dayton SCR motor controller Model $2 \mathrm{M} 171 \mathrm{C}$ which controls the motor direction and output rpm. Power is transferred by a timing belt from an 18 toothed timing gear fixed to the motor drive shaft to a 60 toothed timing gear attached to the 8:1 gear box. The combination of timing belt gears and gear box yields a total reduction ratio of 26.66:1. This large gear reduction was desired to maintain high motor rpm at low pitching rates which contributes to smoother and steadier operation due to increased angular momentum. A crank is fixed to the output end of the gear box which drives an intermediate linkage fixed to the bottom side of the test section floor via a drive rod. This intermediate linkage in turn drives the main drive rod attached to the model. The main drive rod is connected 3.5 inches $(889 \mathrm{~mm})$ aft of the wing rotation point which is located at the midchord position. The intermediate linkage is slotted so the crank and connecting rod can be moved to different locations along its length. This provides a means of changing the 
length of the lever arm, hence changing the angle of attack range of the wing. Different sized cranks can also be used to obtain the same result. The mean angle of attack is selected by raising or lowering the model sting, which causes the model apex to raise or lower.

Pitching rate is controlled by varying the output rpm of the Dayton electric motor using the SCR control. This output rpm was determined using an optical sensing device which directly displayed the motor rpm on an LED readout. It was found that the motor rpm could be held to \pm 3 rpm about a desired nominal value.

Model motion was controlled through the use of an electronic clutch connected to the crank drive shaft. Using the clutch, motion could be started and stopped while the DC motor continued to run. The mechanism could be operated in either a continuous or pulse mode by switching a lever on a control box. Continuous pitching was maintained as long as the lever was switched to continuous mode. Switching the lever to the pulse mode stopped the motion. While in the pulse mode, the model could be pulsed from one angle of attack to another. Upon depression of a button, model motion continued for a specified time interval which was determined by a potentiometer setting on the control box. By varying the time the clutch was engaged, the desired angle of attack range could be obtained. A schematic for the control box is shown in Figure 2.5 .

\subsection{Flow Visualization}

In this study, a major portion of the early research was devoted to developing a practical method of flow visualization suitable for studying the 
complex flow field about a pitching delta wing model. Three flow visualization techniques were investigated and are discussed below. They include,

1) Smoke rake

2) Smoke Sheet

3) Titanium Tetrachloride ( $\left.\mathrm{TiCl}_{4}\right)$ injection.

The first two methods used kerosene smoke which was generated by flash vaporization of deodorized kerosene. This was accomplished by dripping kerosene on electrically heated plates enclosed in conduits through which air was forced by a squirrel cage blower. A sketch of the smoke generator is shown in Figure 2.6.

In the first method, a smoke rake was attached to the kerosene smoke generator which is shown in Figure 2.7. The smoke rake consists of a heat exchanger, filter bag (for eliminating large particles) and smoke tubes through which the kerosene smoke passes. The smoke tubes could be opened and closed to limit the number used. Smoke was introduced into the flow at the tunnel inlet at five evenly spaced vertical locations. The five smoke filaments, each approximately one inch in diameter, spanned roughly 12 vertical inches $(30.5 \mathrm{~cm})$ in the 24 in $\times 24$ in $(61 \mathrm{~cm} \times 61 \mathrm{~cm})$ test section after passing through the wind tunnel contraction.

It was necessary for the smoke to impinge the model apex for proper vortex core and breakdown visualization. The delta wing was oscillated from $0^{\circ}$ to $30^{\circ}$ in the preliminary smoke visualization tests. This implied that the apex traversed approximately 4 vertical inches as it oscillated. The rake was positioned so the upper smoke filaments impinged close to, or on, the apex of the model as shown in Figure 2.8. Therefore, as the apex of the model moved in the vertical plane it would leave one filament behind and pick up the next, thus allowing for proper visualization of the vortex flow. 
This method provided promising results if the smoke rake was positioned at the proper vertical and horizontal locations. However, this was not easily accomplished due to random fluctuations of the smoke filaments within the tunnel test section. As a result, time consuming adjustments were almost continuously needed. As the smoke filaments drifted from the apex position, they would become entrained into the outer vortex feeding sheets causing visual distortion of vortex core and breakdown position.

Another drawback was the spacing between smoke filaments. As the model oscillated there were spaces in the flow field for which there was no smoke. Thus, continuous vortex flow visualization could not be maintained for large amplitude motions.

For large amplitude cases a "smoke sheet" visualization technique was considered. This smoke sheet was produced using the smoke rake in tandem with a mixing device affixed to the smoke rake. This attachment produced a sheet of smoke from the smoke filaments using small mixing vanes which were housed inside. When positioned at the tunnel inlet, a sheet of smoke approximately one inch $(2.54 \mathrm{~cm})$ thick convected down the centerline of the tunnel to impinge on the model apex.

Again, there were problems with alignment of the smoke due to the "sheet" drifting in the tunnel. The "sheet" might impinge the apex at one point in the cycle, and miss it at another. This occurred because the "sheet" did not stay vertical. It had a tendency to twist off centerline as it convected down the tunnel. Because of the improper smoke location, the visualization of the vortex core would become obscured due to the entrainment of smoke into the outside feeding sheets of the vortex. This made the position of vortex breakdown hard to locate. Also, the smoke was less dense due to the increased volume of the 
"sheet" compared to the smoke filaments. This resulted in lower quality flow visualization.

The major problem in the above two techniques was the lack of control over the smoke position. Therefore a completely different method was investigated. Instead of introducing smoke at the tunnel inlet, smoke was introduced into the flow field at the model apex. This was accomplished using $\mathrm{TiCl}_{4}$ vapor which produces a dense white smoke when exposed to the moisture in the atmosphere.

$\mathrm{TiCl}_{4}$ vapor was pumped from a specially designed thick-walled glass container using pressurized nitrogen gas. Nitrogen gas is an inert element with which $\mathrm{TiCl}_{4}$ does not react. A schematic of the system is shown in Figure 2.9. At standard room conditions, the liquid in the reservoir is easily vaporized because of the chemical's low vapor pressure. Therefore, as nitrogen is passed through the glass container, $\mathrm{TiCl}_{4}$ vapor is taken with it. A piece of $3 / 16$ in $(4.76 \mathrm{~mm})$ I.D. tubing was used to deliver $\mathrm{TiCl}_{4}$ vapor to the model with a $1 / 16$ in $(1.59 \mathrm{~mm})$ stainless steel probe attached to the end. The flow of vapor was regulated using a needle valve located upstream of the $\mathrm{TiCl}_{4}$ container.

The probe was placed at various surface locations around the model apex to find the position which produced the best quality flow visualization of the vortex core and breakdown location. After location of the optimal position, the probe was placed inside the model at that point, with the probe tip flush to the model's surface. This eliminated any interference affects caused by an externally mounted probe. The $\mathrm{TiCl}_{4}$ supply tube was housed in a channel on the underneath side of the wing (again to limit interference effects). From there, the supply tube exited the test section along the main drive rod. As the model oscillated, a continuous stream of particles was ejected into the flow at the same 
position relative to the model. This insured continuous quality flow visualization at all angles of attack within the cycle.

The density of the smoke was adjusted by changing the flow rate. If the flow rate was too low, the smoke would only faintly show up in the high speed movies. If the flow rate was too high, too much smoke was produced causing the core to appear larger on the film. This made locating the position of vortex breakdown more difficult because the flow expansion at breakdown was not as clear as it was when less smoke was used. Therefore, a flow rate between the two was used which insured good quality flow visualization. This rate was not quantified, and in each case refining adjustments were made to it from qualitative visual observations. The effect of the flow rate on the vortex flow was also investigated. The flow rate did not seem to effect the location of vortex breakdown, even when it was increased well beyond the settings used for data acquisition.

$\mathrm{TiCl}_{4}$ vapor was by far the best method for visualizing the flow over a pitching delta wing. However, it was not without its drawbacks. The dense white smoke produced upon contact with water vapor in the atmosphere is a highly toxic and corrosive mixture of hydrochloric gas and minute titanium oxide particles. The resulting hydrochloric gas is orderless and tasteless, and caution should be used when working with it. Proper ventilation and exhaust is vital. The equipment should also be periodically checked for corrosive damage.

After prolonged use, titanium oxide particles tend to build up on the model surface at the probe location, and periodic cleaning is required. It is also important to note that if too small of tygon tubing is used to deliver the $\mathrm{TiCl}_{4}$ vapor, clogging can occur. Initially, 1/16 in (1.58 mm) tubing was used, and clogging periodically occurred. Later, $3 / 16$ in $(4.8 \mathrm{~mm})$ I.D. tubing was used, and clogging did not occur. 


\subsection{Photographic Records}

After having developed a suitable means for injecting tracer into the vortex flow field, two systems were developed to acquire photographic data. This was a critical aspect of the research because all the data presented on vortex flow came solely from visual data. The systems used both $16 \mathrm{~mm}$ high speed cinema photography and $35 \mathrm{~mm}$ Black and White (B \& W) still photography.

The first system used a Milliken DMB-5 $16 \mathrm{~mm}$ motion picture camera for the high speed cinema photography. A film frame rate of 64 frames per second (effective shutter speed $1 / 160$ second) was used with Eastman $16 \mathrm{~mm} 4-X$ high speed movie film. The lens used was a P. Angenieux 1:1.5 set at an aperture opening of $\mathrm{f} / 2.8$. The model and smoke flow was illuminated using a $1000 \mathrm{Watt}$ flood lamp placed above and upstream of the test section and the camera was positioned perpendicular to the pitching axis to provide a side view of the flow field. A schematic of the setup is shown in Figure 2.10. By taking motion pictures from the side, instantaneous angle of attack, chordwise vortex breakdown position, and vortex inclination angle data was acquired. A timing light was placed in the field of view of the motion picture camera and used to indicate the start of each pitching cycle. The light was triggered by a microswitch on the gearbox which was engaged at a specific crank position.

The second method incorporated the use of two Nikon FM2, 35mm SLR cameras with Nikon MD-12 auto winders and a 1000 Watt flood lamp located diagonally forward and above the model as shown in Figure 2.11. One camera was positioned perpendicular to the pitching axis (as for the motion picture camera) to provide a side view of the flow and the other was positioned parallel to the pitching axis and directly above the test section to provide a top view. 
The shutters on both cameras were simultaneously released using a solenoid device. The solenoid was connected to the control box (discussed in Section 2.3) and triggered by the micro-switch on the gear box. From the two views, three-dimensional data on the flow field was obtained. The data acquired, supported data extracted from the high speed motion pictures as well as providing additional information on the spanwise position of vortex breakdown.

Kodak Tri-X pan, 400 ASA B \& W print film was used to obtain the still photographs. The shutter speeds were set at $1 / 250 \mathrm{sec}$ on both cameras. The lens on the side mounted camera was a Nikon Nikkor $50 \mathrm{~mm} 1: 1.4$ set at an aperture opening of $\$ / 2.0$, and the lens on the top mounted camera was a Nikon

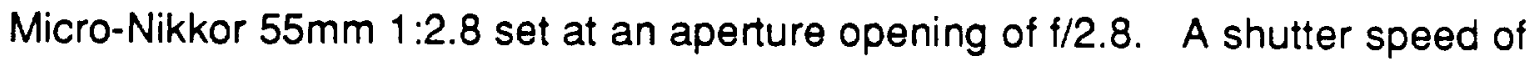
$1 / 250 \mathrm{sec}$ was the fastest speed attainable while keeping exposure levels at an acceptable level.

\subsection{Hotwire Anemometry}

Freestream flow measurements where conducted to investigate the effects of model motion on the freestream flow. Freestream flow velocities were obtained using a hot wire probe placed in the center of the test section, 31 in $(78.74 \mathrm{~cm})$ upstream of the model sting or 5 in $(12.7 \mathrm{~cm})$ into the $6 \mathrm{ft}(182.9 \mathrm{~cm})$ tunnel test section. A Dantec Type P11 probe was used and mounted in a Dantec Type 55H20 support and right angle mounting tube.

The experiment used a TSI Intelligent Flow Analyzer 100 (IFA 100) Model 158 anemometer operated in constant temperature mode. A built-in signal conditioner was used to provide a DC bias to the output signal. Typically the output signal, before any conditioning, varied from 1 to 2 volts (overheat ratio of 1.8$)$ for velocities from 0 to $35 \mathrm{ft} / \mathrm{s}(10.7 \mathrm{~m} / \mathrm{s})$. A DC offset of $-2 V$ was 
applied using the signal conditioner to place the output signal in a range of $-1.25 \vee$ to $+1.25 \vee$ which was needed for the Digital Equipment Corporation DT-2752 A/D converter.

Tests conducted on the freestream response due to model motion for the lowest amplitude case revealed an approximate $0.5 \%$ deviation from the mean freestream velocity. This small velocity fluctuation accounted for roughly 60 of the 4095 discrete voltage steps usable from the A/D converter. To increase resolution, the gain was increased from 1 to 2 on the signal conditioner. The gain could only be increased in whole number increments. This effectively doubled the resolution of the output signal. If the gain was further increased to 3 , the resulting output voltage was too great for the specified range of the A/D. While using the gain of 2 , the $D C$ offset was set at $-1 \mathrm{~V}$.

The output signal was filtered with the TSI internal signal conditioner at one-half the sampling rate prior to data acquisition. The filter was a low-pass filter with a $1-500 \mathrm{kHz}$ range, a rolloff of $18 \mathrm{db} /$ octave, and an accuracy of $10 \%$. For these test however, filtering was not critical due to the low frequencies involved which ranged from 0.2 to $1.0 \mathrm{~Hz}$.

\subsection{Displacement Transducer}

To provide detailed information on the model motion, a Trans-Tek Series 240. Model 0245-0000 displacement transducer was used to provide position data. Tests performed with the instrument provided a means of measuring harmonic distortion in the sinusoidal pitching motion and phase difference between the model motion and freestream response. Phase difference was obtained by comparing simultaneous output from the transducer and hot wire anemometer. 
The transducer is an integrated package consisting of a linear variable differential transformer, a solid state oscillator, and a phase-sensitive demodulator. A schematic is shown in Figure 2.12. The oscillator converts a $D C$ input to $A C$, which excites the primary winding of the differential transformer. Voltage is induced in the secondary winding by the axial core position. The circuits are connected in series opposition so that the resultant output is a DC voltage linearly proportional to core displacement from the electric center, which is the position at which the output voltage is $0 \mathrm{~V}$. The polarity of the voltage is a function of the direction of the core displacement with respect to the electric center. Therefore, output voltage was linearly proportional to model displacement.

The displacement transducer was rigidly fixed to the support structure, and its core attached to the intermediate linkage of the drive mechanism. As the model oscillated, the core was free to move in the axial direction. The device was calibrated plotting output voltage from the transducer against angle of attack. Thus, angle of attack information was obtained from the voltage output.

The input voltage and transducer position were varied until the output voltage ranged from $-1.0 \mathrm{~V}$ to $1.0 \mathrm{~V}$ throughout it range of motion. This was done to use a majority of the discrete voltage steps available from the AVD converter as mentioned in the previous section (effective range of $-1.25 \mathrm{~V}$ to $+1.25 \mathrm{~V})$. The input voltage was approximately $5.0 \mathrm{~V}$ for the small amplitude cases and $4.0 \mathrm{~V}$ for the large amplitude cases.

The output signal from the transducer was conditioned using the signal conditioner on the TSI Intelligent Flow Analyzer (see previous section). Again. the signal was low-pass filtered at half the sampling rate prior to data acquisition. When position and freestream velocity data were simultaneously 
sampled, signal conditioning of each input was identical. This eliminated the possibility of a phase shift being caused by a difference in signal conditioning.

\subsection{Data Acquisition Systems}

Two data acquisition systems were used to collect and store information. One consisted of a Digital Equipment Corporation (DEC) PDP-11/23 and an 8 channel, 12 bit, Data Translation DT-2752 analog to digital (AVD) converter. It was used to collect data from the hot wire anemometer and displacement transducer. The system was also equipped with two Schmitt triggers for conditional sampling applications. The other system consisted of a Wayne George Optical Comparator Interfaced with a Digital Equipment Corporation (DEC) DRV-11, and used to digitize photographic data. Data reduction was accomplished using a PDP-11/34 with a DEC RT-11 operating system and DEC FORTRAN IV V2.5. Plotting the data was accomplished using a HewlettPackard model 7470A plotter or a Apple Computer Macintosh System. 
CHAPTER III

PROCEDURE AND DATA ACQUISITION

Four different types of experiments were performed in this study. They included:

1) the determination of harmonic distortion in the sinusoidal pitching motion of the model,

2) the investigation of the freestream response due to varying tunnel blockage resulting from model motion,

3) the recording of the breakdown location using high speed photography,

4) the recording of the breakdown location using synchronous $35 \mathrm{~mm}$ photography.

After collection of the photographic data, a detailed procedure was used to reduce the data. A complete description of the device and the technique involved will be presented after descriptions of the experimental procedures.

\subsection{Model Motion}

To obtain a value for harmonic distortion in the sinusoidal pitching motion, the linear variable differential transformer (LVDT) was used. The LVDT 
was calibrated by plotting output voltage against model angle of attack. Angle of attack was measured with an inclinometer, which had an accuracy of approximately $\pm 0.25^{\circ}$, at 10 equally spaced angle of attack locations throughout the range of motion. At each position, the angle of attack and the output voltage were recorded and a calibration curve was constructed.

The Digital Equipment Corporation PDP 11/23 lab computer was used to sample data from the LVDT. In each case, 32 cycles of 32 points each were sequentially sampled at a rate which was determined by pitching frequency. A Discrete Fourier Transform (DFT) was performed on the 1024 data points, and amplitude was plotted against frequency. By comparing the amplitudes of the harmonic frequencies with the amplitude of the fundamental frequency, a value for harmonic distortion was obtained. This calculation will be further explained in Chapter IV. It was not necessary to change the output voltage to angle of attack because the resulting calibration curve was linear. Therefore the output voltage could be used directly to determine the harmonic distortion.

\subsection{Freestream Response}

To determine the effect of the model motion on the freestream flow, data from the LVDT and hot wire anemometer was simultaneously sampled using the PDP 11/23 computer. In each case, one hundred data records were taken, each starting at the same position in the pitching cycle. This was accomplished through the use of the Schmitt trigger and the 11/23 Computer. The trigger was activated by a voltage impulse originating from a micro switch that was engaged at a specific crank location. Each record contained two cycles of 20 points each, taken at a sample rate based on frequency of oscillation. The data from the 100 records were ensemble averaged and the percent difference in the 
freestream velocity from the mean value and the relative model angular position were plotted against the period of oscillation for the two cycles. From the plots, a value of phase angle between the model motion and freestream response was obtained though graphical analysis. The technique will be discussed in Chapter IV.

\subsection{High Speed Motion Picture Data}

Static data on the chordwise position of vortex breakdown was obtained for both increasing and decreasing angle of attack for use as a comparison to the dynamic cases. The positon of vortex breakdown was recorded with the Millikin DMB-5 high speed motion picture camera. The vortex breakdown location was visualized using the $\mathrm{TiCl}_{4}$ tracer, and a 1000 Watt flood lamp was used to provide illumination. The camera was located approximately $8 \mathrm{ft}<2.44$ m) from and parallel to the glass plate composing the side wall of the test section, and centered on the pitching axis of the model to provide a side view of the flow. Data was recorded on Eastman $16 \mathrm{~mm} 4-X$ negative movie film at a film rate of 64 frames/sec with a lens aperture setting of $t / 2.8$.

The model was placed at its minimum angle of attack within the range of motion, and the freestream velocity increased to $30 \mathrm{tt} / \mathrm{s}$. Data was recorded at the minimum angle of attack and at 8 increasing incremental angles of attack until the maximum value was attained. Approximately 2 seconds of data was obtained at each angle of attack, corresponding to about 120 frames of movie film. The amount of time between the incremental measurements was at least 20 seconds. It was felt that this was adequate time for the flow to reach a steady state. At each angle of attack, an average chordwise breakdown location was computed from 10 equally spaced movie frames which spanned approximately 
1.5 seconds of data. The average chordwise position of vortex breakdown was then calculated, and plotted against angle of attack. The procedure was then repeated for decreasing angle of attack.

The procedure for the dynamic case was basically the same with the motion picture camera and lighting setup the same. After setting the freestream velocity, pitching rate, and angle of attack range parameters, photographic records were taken at a film rate of 64 frames/sec. In each case, the response of the vortex flow was recorded for at least 8 complete cycles of motion. The data was then reduced and plotted as for the static case.

\section{$3.435 \mathrm{~mm}$ Photographic Data}

The spanwise position of vortex breakdown was acquired using two Nikon FM2 $35 \mathrm{~mm}$ SLR cameras simultaneously triggered by a micro-switch on the drive mechanism which was engaged at a specific crank position. One camera was used to provide angle of attack information and the other was used to record spanwise breakdown location. The camera which provided angle of attack information was located approximately 24 in $(61 \mathrm{~cm})$ from the glass plate composing the side wall of the test section, and centered on the pitching axis of the model. The camera used to record the spanwise breakdown location was located approximately 20 in $(51 \mathrm{~cm})$ from the glass plate on top of the test section, and centered on the axis of the model sting. The shutter speeds were $1 / 250 \mathrm{sec}$ for both cameras, and the lens settings were $\$ / 2.0$ and $t / 2.8$ for the side and top cameras respectively. These settings were considered to be optimal based on the results of test films in which a wide variety of lens openings and shutter speeds were used. 
Photographs were taken at 4 locations during the pitching cycle which included maximum amplitude, minimum amplitude, and mean angle of attack during the upstroke and the downstroke. These angular positions were determined using a strobe light which was connected in parallel with the camera solenoid. When a signal was sent to release the camera shutters, the strobe light flashed. The flash was used to "freeze" the crank position, and by adjusting the time delay between the engagement of the micro-switch and the release of the shutters, any crank position could be selected. By selecting the crank position, at which the shutters were released, the vortex flow at the desired angles of attack could be recorded. The crank position relating to the desired angles of attack were $0^{\circ}, 90^{\circ}, 180^{\circ}$ and $270^{\circ}$. At each of the 4 locations, 5 sets of photographs were taken to be used for ensemble averaging. One 1000 Watt flood lamp was used for illumination, and $\mathrm{TiCl}_{4}$ was used to visualize the vortices. The flood lamp was placed diagonally forward and above the model.

\subsection{Reduction of Photographic Data}

Because a majority of the data in this study comes from reduced photographic data, a detailed description of the equipment and process involved is presented in the following sub-sections. An estimation of the accuracy for the equipment and reduced data will also be given.

\subsubsection{Wayne-George Optical Comparator}

Data reduction was accomplished using a Wayne-George Optical Comparator. The Wayne-George Optical Comparator consists of a 36 in $\times 36$ in 
$(91 \mathrm{~cm} \times 91 \mathrm{~cm})$ digitizing table and a cabinet which contains the power supply, the data display, and associated hardware called the "bi-directional counter." The device is used to record " $x$ " and " $y$ " coordinates at selected locations on the digitizing table. A data point is recorded by positioning a target mechanism (constrained to move in the " $x$ " and " $y$ " direction) over the point of interest. The data point coordinates are then sent to the Wayne-George "bi-direction counter" upon depression of a foot pedal trigger, and displayed on an " $x$ " and " $y$ " LED coordinate readout. This display gives the coordinates in inches relative to a zero-position set by the operator. The resolution of the reading is 0.002 in $(0.051 \mathrm{~mm})$.

\subsubsection{Wayne-George to DEC Interface}

In order to collect and store data, the Wayne-George was interfaced with a Digital Systems Equipment (DEC) DRV-11 laboratory computer for data storage and manipulation. This was done using an interface board designed by Phillip G. Corporon, Digital Computer Specialist at the University of Notre Dame.

The Wayne-George uses an outmoded data protocol and abnormal input/output voltage levels. The non-standard input/output voltage logic levels are set at $O$ VDC for a logic of 0 and at +12 VDC for a logic of 1 . The DRV-11 uses the application of a standard convention configured for both the standard transistor-transistor logic (TTL) levels and the parallel binary format (Corporon, 1987).

The problem with the DRV-11 is that it can handle only one data word at a time, compared to the Wayne-George simultaneous output of two data words. Thus, the interface board that was designed, transformed the Wayne-George 
data format and voltage levels to an acceptable form for the DRV-11, and converted the transmission of two simultaneous data words into two sequential data words.

\subsubsection{Wayne-George Accuracy}

The accuracy of the Wayne-George is addressed in this section, and should not be confused with the accuracy of the readings taken from the photographic images, to be discussed in a later section. To test the accuracy of the Wayne-George Optical Comparator, three experiments were performed in order to determine:

1) the repeatability of a measurement (the human factor),

2) hysteresis,

3) distortion.

The first test was conducted using a small "dot" placed on the digitizing table. The "dot" was approximately the same diameter as the 0.020 in target mechanism pointer. The position of the "dot" was recorded, the pointer moved away, and then recorded again. The process was repeated 100 times to provide statistical information on the repeatability of a single point. As mentioned, the Wayne-George itself has an resolution of 0.002 in. From the test, a rms of 0.005 in was calculated in the position measurement for the 100 data point sample. The maximum error from the sample was $0.017 \mathrm{in}$. The rms provided an indication of the average human error involved in recording data. It is important to realize that these results come from that of a well-accustomed Wayne-George operator and results may vary from person to person. It is also important to note that the location of a "dot" was digitized in this experiment. Unfortunately, the points of interest in the photographic images are not so well 
defined and subjectivity becomes a larger factor which leads to increased experimental error.

The second experiment tested for hysteresis inherent to the machine. To accomplish this, a horizontal reference line was placed across the center of the digitizing table. The pointer on the target mechanism was moved to the limit of its travel in the negative " $y$ " direction (the bottom of the table). It was then moved directly to a point on the reference line, and the $y$-value recorded. The pointer was then moved to the limit of its travel in the positive "y" direction and again directly back to the reference, and the $y$-value recorded. This process was repeated 25 times. The average of the $y$-values coming from the negative side was compared with the average of the $y$-values coming from the positive side. A difference in the averages would indicate a hysteresis in the recording device. The experiment was performed along the $x$-axis using a vertical reference line as well. The differences among the respective averages were within the value of human resolution, indicating no measurable hysteresis in either direction.

The third test was performed to determine if there was distortion in the Wayne-George coordinate system. To test for this, 5 concentric rectangles were drawn with the largest being approximately 8 in $\times 10$ in. The corners on each rectangle were digitized and the coordinates recorded. The rectangles were then plotted and checked for distortion by using a drawing square. The results indicated that no detectable distortion exists.

\subsubsection{Data Acquisition with the Wayne-George}

The Wayne-George/DRV-11 operating system was used extensively for data acquisition because of the ease with which coordinate information could 
be collected and stored. All the data presented on vortex flow, came from photographic data which was digitized using the Wayne-George system. Two types of images were digitized. One provided a side view of the flow field, the other a top view of the flow field. The digitizing procedures for both are explained below.

Sideviews of the flowfield were obtained using high speed motion pictures and $35 \mathrm{~mm}$ still photography, for which the digitizing procedure was the same. A typical photograph of the image is shown in Figure 3.1, and a schematic of the image (for discussion purposes) is shown in Figure 3.2. This image was projected onto the digitizing table using 3 plate glass mirrors arranged as shown in Figure 3.3. To project the movie film, a Lafayette Analyzer Projector Model 00300 was used; and to project the $35 \mathrm{~mm}$ negatives, a photographic enlarger was used. The resulting image, projected on the digitizing table, was approximately 18 in $\times 14$ in $(46 \mathrm{~cm} \times 36 \mathrm{~cm})$. Five points (labeled A-E on the schematic in Figure 3.1) were digitized to yield angle of attack, chordwise vortex breakdown location,vortex breakdown height, and vortex inclination angle $(\beta)$. The two points $(A$ and $B$ ) were digitized at positions along the downstream side of the model sting in order to define a vertical reference vector, and the two points ( $C$ and $D$ ) were digitized at locations corresponding to the model apex and the center of the trailing edge respectively. The points ( $C$ and $D$ ) defined a vector lying along the root chord of the model. The center of the trailing edge was located by using a small "pin-like" probe which extended approximately $1.5 \mathrm{in}(3.8 \mathrm{~cm})$ aft of the trailing edge along the root chord line. It was necessary to use the probe because the midline of the trailing edge is indistinguishable in a side view of the model. The length of the probe was then subtracted from the $C D$ vector to define the 
magnitude of the root chord. The dot product was used with vectors $A B$ and $C D$ to defined angle of attack as shown in the equation below.

$$
\alpha=\frac{\pi}{2}-\cos ^{-1}\left(\frac{\overrightarrow{A B} \cdot \overrightarrow{C D}}{|\overrightarrow{A B}||\overrightarrow{C D}|}\right)
$$

$A$ fifth and final point $E$ was then taken at the leading edge vortex breakdown location. The point was defined as the position at which the vortex core first appears to expand. This point was the most difficult of the five to accurately locate due to its blurred appearance. The accuracy with which one could pinpoint the location of vortex breakdown varied from frame to frame, and from film to film. In some frames a distinct expansion of the flow was clearly visible, while in others the expansion was more subtle. Some films had poorer smoke quality than others making the vortex core and breakdown location more difficult to see. These factors contributed to a less accurate burst location measurement. Obviously, the measurement was somewhat subjective. However, after digitizing thousands of points, the experimenter becomes somewhat consistent in locating the breakdown location, and any bias that he may add is the same from frame to frame.

Using point $E$, values for the vortex inclination angle $(\beta)$, the nondimensional chordwise burst location $(x / c)$, and the nondimensional height of the burst $(z / c)$, were calculated. The vortex inclination angle was determined using the rootchord vector $C D$ and the newly defined vector $C E$ which represented the vortex core. The value for $x / c$ was calculated by dividing the projection of vector $C E$ on vector $C D$, by the magnitude of the root chord vector. In a similar fashion, a value for $z / c$ was defined. The equations which were used are shown below. 


$$
\begin{aligned}
& x / c=\cos (\beta) h / c \\
& z / x=\cos (\beta) h / c
\end{aligned}
$$

where,

$$
\begin{aligned}
& \beta=\cos ^{-1}\left(\frac{\overrightarrow{C D} \cdot \overrightarrow{C E}}{|\overrightarrow{C D}||\overrightarrow{C E}|}\right) \\
& h=|\overrightarrow{C E}|, \text { the magnitude of the vortex vector } \\
& c=0.895|\overrightarrow{C D}|, \text { the root chord (considering the TE probe). }
\end{aligned}
$$

Topviews of the flow field were obtained from $35 \mathrm{~mm}$ still photography. A typical photograph is shown in Figure 3.4 and a schematic of the photograph is shown in Figure 3.5. This image was projected onto the digitizing table in the manner as mentioned above. Three points (labeled A-C in Figure 3.5) were digitized on each photograph to provide spanwise breakdown location. All points layed along a line perpendicular to the root chord of the model which passed through the vortex breakdown position. The perpendicular line was defined on the projected image using a drawing square placed along the root chord. The points $A, B$, and $C$, corresponded to points at the root chord, vortex breakdown location, and leading edge. Using vectors $A B$ and $A C$, a value for the nondimensional spanwise location $(y / s)$ of vortex breakdown was obtained.

Because the photographs were taken perpendicular to the freestream and not to the model, compensation for angle of attack was necessary in order to correctly define the chordwise location of the measurement. Because of the short camera focal length involved (which varied with angle of attack), paralax error in the spanwise direction was compensated for as well. These errors were determined using information obtained from a side view photograph, which was taken at the same time of the top view photograph. Therefore, at each angular 
position, it was necessary to digitize both the side and top views to compensate for the paralax error. The equations used to compute $y / s$ are shown below.

$$
y / s=\frac{|\overrightarrow{A B}|}{|\overrightarrow{A C}|}(1-\text { paralax })
$$

where,

$$
\begin{aligned}
& \text { paralax }=\frac{16.5(z / c)}{\text { camhgt }} \\
& \text { camhgt }=32-16.5 \sin (\alpha)(0.5-x / c) \quad \text { camera height }
\end{aligned}
$$

\subsubsection{Accuracy of Reduced Data}

Angle of attack data was used to evaluate the accuracy of the digitized motion pictures. As previously stated, digitization of movie frames began nominally at the same angular position for each cycle, and because the same number of frames were skipped between data frames in each cycle, the time increment between data frames was the same. In actuality, however, the instantaneous angles of attack recorded at the beginning of each cycle could differ by as much as $1 / 64 \mathrm{sec}$, the film rate of the high speed motion picture camera. This corresponded to a maximum of $0.30^{\circ}$ at the fastest pitching frequency and $0.08^{\circ}$ at the lowest, for the angle of attack range of $29^{\circ}$ to $39^{\circ}$ in which most tests occurred. Nominally speaking, however, data frame 1 was recorded at the same angle of attack for each cycle, as well as data frame 2, 3, 4 , and so on. By comparing data frames in this manner throughout the entire run, a measure of relative error was computed. Figure 3.6 shows a representative plot of 9 digitized cycles which have been overlayed starting at the same nominal angle of attack. The plot illustrates how angle of attack varies 
from cycle to cycle at a specific locations within the cycle. The solid line represents the average values. As an indicator of relative error, the rms of the angle of attack was computed for the data set at each of the 17 points. The average rms for the 17 points was $0.223^{\circ}$. A summary of the high speed movie cases is presented in Table 3.1. The Table contains the number of cycles digitized, and the average rms of the angle of attack measurements.

TABLE 3.1

High Speed Movie Cases Digitized

\begin{tabular}{|c|c|c|c|c|c|c|} 
Case & $\begin{array}{c}\alpha \text { range } \\
\text { (deg) }\end{array}$ & $\begin{array}{c}u \\
(\mathrm{ft} / \mathrm{s})\end{array}$ & $k$ & \#cycles & \#points & $\begin{array}{c}\mathrm{rms} \\
(\mathrm{deg})\end{array}$ \\
1 & $29-39$ & 30 & 0.05 & 8 & 17 & 0.19 \\
2 & $29-39$ & 30 & 0.10 & 8 & 17 & 0.22 \\
3 & $29-39$ & 30 & 0.20 & 20 & 17 & 0.22 \\
4 & $29-39$ & 30 & 0.30 & 9 & 17 & 0.20 \\
5 & $29-39$ & 10 & 0.20 & 9 & 16 & 0.17 \\
6 & $29-39$ & 20 & 0.20 & 12 & 17 & 0.18 \\
7 & $29-39$ & 30 & 0.20 & 20 & 17 & 0.22 \\
8 & $29-39$ & 40 & 0.20 & 8 & 16 & 0.36 \\
9 & $0-45$ & 30 & 0.05 & 10 & 17 & 0.29 \\
10 & $0-45$ & 30 & 0.30 & 10 & 17 & 0.45 \\
11 & $29-39$ & 30 & static-up & - & $10 /$ aoa & - \\
12 & $29-39$ & 30 & static-dn & - & $10 /$ aoa & -
\end{tabular}

The average relative error for all the cases based on rms data was $0.24^{\circ}$. This measure contains the errors induced by the $\pm 1 / 64$ degree accuracy of the high speed motion picture camera and also the error in digitizing procedure. 
Based on the size of the projected images, the overall rms of $0.24^{\circ}$ corresponds to a maximum distance error of 0.078 in $(2 \mathrm{~mm})$.

The error in the breakdown location measurement was not as easily defined. As mentioned previously, the actual position of vortex breakdown was a subjective evaluation. In some frames a distinct expansion of the flow was clearly visible, while in others the expansion was more subtle. However as the experimenter becomes accustomed to the phenomenon, the subjectivity of the measurement decreases. Taking these factors into consideration, the degree of accuracy in the breakdown location measurement is estimated at $0.01 \times / c$, or approximately 0.16 in $(4 \mathrm{~mm})$ on the projected image.

The above value should not be confused with the accuracy of the ensemble averaged cycle-to-cycle variation of breakdown location. The ensemble average of the breakdown location is the average of all the breakdown position measurements taken at specific angles of attack in each of the recorded cycles. The breakdown location fluctuates in a random fashion (approximately $\pm 0.04 \times / c$ ) as it propagates up and down the model due to the pitching motion. This random fluctuation in burst position is illustrated in Figure 3.7 where 9 cycles of motion are overlayed on one plot for a reduced frequency of $k=0.30$ and a freestream velocity of $30 \mathrm{ft} / \mathrm{s}(R e=260,000)$. Notice the fluctuation in the breakdown location at a specific angle of attack. Therefore, the more cycles which are ensemble averaged, the more representative the data. As seen in the Table 3.1 above, the number of cycles averaged varied from 8 to 20, with the average being about 10. This limitation in the number of cycles was due to available film footage ( $100 \mathrm{ft}$ per roll). It was felt that 8 cycles provided a good representation for analysis of the flow, based on tests which investigated what effect the number of cycles had on the average. The cases 
with a higher number of cycles were used for additional statistical analysis, and will be discussed Chapter IV.

No detailed analysis was conducted on the accuracy of the reduced $35 \mathrm{~mm}$ data because only a limited number of photographs were digitized. Ensemble averaging of the data had been planned, but due to unexpected problems (discussed in Section 4.6), only one data point was recorded at each specific angle of attack. However, the data does provide an indication of how the breakdown location varies in the spanwise direction, and the error involved should be approximately equal to that of the motion picture data. 


\section{CHAPTER IV \\ RESULTS}

\subsection{Introduction}

To investigate the response of the leading edge vortices and chordwise breakdown location while undergoing oscillatory motion, the following tests were performed. The first of which determined the effect of reduced frequency on the vortex breakdown location. The freestream velocity was held constant at $30 \mathrm{ft} / \mathrm{s}$, corresponding to a freestream Reynolds number of 260,000 , for reduced frequencies of $k=0.05,0.10,0.20$, and 0.30 . The wing was sinusoidally pitched between $29^{\circ}$ and $39^{\circ}$ about the $1 / 2$ chord location. Throughout this angle of attack range, vortex breakdown occurred over the upper surface of the wing.

The second set of tests investigated the effect of Reynolds number on the vortex breakdown location at a fixed reduced frequency of $k=0.20$. The Reynolds number was varied between 90,000 and 350,000 , and specific tests were conducted for freestream velocities of $10,20,30$, and $40 \mathrm{ft} / \mathrm{s}$. The angle of attack range was again $29^{\circ}$ to $39^{\circ}$.

Another set of tests studied the effects of a larger amplitude motion on the vortex flow. The wing was pitched from $0^{\circ}$ to $45^{\circ}$ at two reduced frequencies of $k=0.05$ and 0.30 . The freestream velocity was $30 \mathrm{ft} / \mathrm{s}$ which corresponded to a freestream Reynolds number of 260,000 . In these tests, the processes of flow 
separation and reattachment were analyzed in addition to vortex breakdown location.

Static tests were also performed to provide a comparison with the dynamic cases. The vortex breakdown position was recorded at static angles of attack between $29^{\circ}$ and $39^{\circ}$. The freestream velocity was $30 \mathrm{ft} / \mathrm{s}$ which corresponded to a Reynolds number of 260,000 . Data was recorded for both increasing and decreasing angles of attack. Statistical information on the oscillation of vortex breakdown was also acquired.

Another type of test was performed to investigate the spanwise position of vortex breakdown during sinusoidal pitching. Tests were conducted at a freestream velocity of $30 \mathrm{ft} / \mathrm{s}$ corresponding to a Reynolds number of 260,000 , for reduced frequencies of $k=0.05,0.20$ and 0.30 The angle of attack range was $29^{\circ}$ to $39^{\circ}$.

A final test was conducted in order to determine the effects of an impulsive change in angle of attack on the vortex flow. The wing was impulsively pitched-up from $28^{\circ}$ to $37^{\circ}$, and impulsively pitched-down from $37^{\circ}$ to $28^{\circ}$. The pitching rate was approximately constant at $0.7 \mathrm{rad} / \mathrm{s}$, and the freestream velocity was $30 \mathrm{ft} / \mathrm{s}$ which corresponded to a Reynolds number of 260,000 .

Before the dynamic vortex flow data was gathered, test conditions were evaluated. Tests were conducted to determine the accuracy of the sinusoidal oscillations, and to quantify the changes in freestream velocity due to the varying tunnel blockage as a result of the oscillating model. 


\subsection{Model Motion}

To obtain a value of harmonic distortion in the sinusoidal pitching motion of the model, a linear variable differential transformer (LVDT) was used. The location at which the LVDT was attached to the pitching mechanism is schematically shown in Figure 4.1. The LVDT was calibrated by plotting output voltage against angle of attack for the small amplitude case of $5^{\circ}$ in which the angle of attack range was $29^{\circ}$ to $39^{\circ}$. The resulting calibration curve is shown in Figure 4.2. The curve was linear within the $\pm 0.25^{\circ}$ accuracy of the inclinometer. It was therefore assumed that the output voltage was directly proportional to angle of attack.

To determine the harmonic distortion, a Discrete Fourier Transform (DFT) was performed on the sampled data which came directly from the LVDT. Calibration was not required because the output voltage from the LVDT was directly proportional to angle of attack. It was only important that the signal be representative of the motion, and it did not matter if was arbitrarily scaled. In each case, output voltage from the LVDT was sequentially sampled for 32 cycles of 32 points each for a total of 1024 data points. Before the DFT was performed, the DC part of the signal was eliminated in order to increase the resolution of the DFT amplitude. This was accomplished by subtracting the average of the data set, from each data point. The DFT was then performed, and the frequency content of the signal obtained. Four tests were conducted which covered the limits of the experimental conditions. The DFT results can be seen in Appendix A. Percent harmonic distortion was calculated by dividing the sum of the first 3 harmonic amplitudes by the amplitude of the fundamental frequency. The results are presented in Table 4.1 
TABLE 4.1

Harmonic Distortion $\left(29^{\circ}\right.$ to $\left.39^{\circ}\right)$

\begin{tabular}{|c|c|c|c|c|c|c|}
\hline$\underset{(t / s)}{U}$ & $k$ & (fund) & $\begin{array}{l}a m p \\
\text { 1st }\end{array}$ & $\begin{array}{l}\text { litude } \\
\text { harmenic } \\
\text { 2nd }\end{array}$ & 3rd & $\begin{array}{c}\% \text { harmonic } \\
\text { distortion }\end{array}$ \\
\hline $\begin{array}{l}30 \\
30\end{array}$ & $\begin{array}{l}0.05 \\
0.30\end{array}$ & $\begin{array}{l}1.59 \\
1.59\end{array}$ & $\begin{array}{l}0.0256 \\
0.0233\end{array}$ & $\begin{array}{l}0.0055 \\
0.0069\end{array}$ & $\begin{array}{l}0.0025 \\
0.0023\end{array}$ & $\begin{array}{l}2.11 \\
2.04\end{array}$ \\
\hline $\begin{array}{l}10 \\
40\end{array}$ & $\begin{array}{l}0.20 \\
0.20\end{array}$ & $\begin{array}{l}1.59 \\
1.59\end{array}$ & $\left|\begin{array}{l}0.0250 \\
0.0240\end{array}\right|$ & $\left|\begin{array}{l}0.0068 \\
0.0067\end{array}\right|$ & $\mid \begin{array}{l}0.0017 \\
0.0023\end{array}$ & $\begin{array}{l}2.10 \\
2.08\end{array}$ \\
\hline
\end{tabular}

Two additional tests were performed for the large amplitude case of $0^{\circ}$ to $45^{\circ}$ at reduced frequencies of $k=0.05$ and $k=0.30$ and a freestream velocity of $30 \mathrm{ft} / \mathrm{s}$. The percent harmonic distortion was computed in the same manner as above. The results indicate a harmonic distortion of $4.34 \%$ for the $k=0.05$ case and $4.50 \%$ for the $k=0.30$ case. These DFT results are also presented in Appendix A. It is important to realize however, that for the large amplitude case, the linear relationship between output voltage and angle of attack becomes less accurate due to the increased angular motion of the drive linkages. The amount by which the calibration curve deviated from the linear relationship was not quantified, and therefore, the results for the large amplitude case should be viewed as approximations.

\subsection{Freestream Response}

The response of the freestream flow, due to the varying tunnel blockage as a result of model motion, was investigated to quantify the velocity fluctuations in the wind tunnel test section, and to determine the phase difference between 
freestream velocity and model motion. To obtain this information, hot wire and LVDT data were sampled simultaneously. Tests were conducted which covered the limits of the experimental conditions. Reduced frequencies of $k=$ 0.05 and 0.30 were investigated at a freestream velocity of $30 \mathrm{tt} / \mathrm{s}$ corresponding to a Reynolds number of $R \theta=260,000$ for the angle of attack ranges of $29^{\circ}$ to $39^{\circ}$, and $0^{\circ}$ to $45^{\circ}$. Also, freestream velocities of 10 and $40 \mathrm{tt} / \mathrm{s}$ hcorresponding to Reynolds numbers of $R e=90,000$ and 350,000 were investigated at a reduced frequency of $k=0.20$ over the angle of attack range of $29^{\circ}$ to $39^{\circ}$.

One hundred data records were taken, each starting at the same point in the oscillation cycle. Each record contained two cycles of 20 points each, taken at a sample rate based on the frequency of oscillation. The reduced data is an ensemble average of the 100 data records. Ensemble averaging was used to average out the freestream fluctuations due to atmospheric disturbances. The effect of the ensemble averaging can be seen in Figures 4.3a and $b$, where one data record is compared to the ensemble average of 100 data records at the same conditions. The percent deviation from mean velocity was plotted against time for two cycles, along with the relative angular model position.

Representative results for each case can be seen in Appendix $B$.

In each case, the velocity deviated from the mean velocity in an oscillatory fashion similar to that of the model motion, however the model and the freestream were not in phase. The freestream fluctuations were relatively small, with the average percent rms of the freestream velocity being $0.37 \%$ for the small amplitude cases of $5^{\circ}$, and $0.92 \%$ for the large amplitude cases of $22.5^{\circ}$.

The percent increase and decrease from mean velocity was approximately symmetric in the small amplitude cases. In the large amplitude cases however, an asymmetry was apparent. The change in velocity at the 
minimum angle of attack of $0^{\circ}$ was less abrupt than at the maximum angle of attack of $45^{\circ}$. This was due to an increase in projected frontal area, caused by the model mount, at low angles of attack. Throughout most of the cycle the mount is "masked" by the projected frontal area of the delta wing. However, as the angle of attack decreases towards $0^{\circ}$, the mount becomes "visible" and produces additional projected frontal area reducing the rate at which the freestream velocity is increasing.

The phase difference between the model motion and freestream response was calculated by measuring the time difference between the point of maximum angle of attack and minimum freestream velocity. This was done graphically, and the maximum and minimum points were estimated visually. The results are an average of two phase differences (one for each cycle of motion) taken from each plot. The velocity phase lag increases with reduced frequency for a constant freestream velocity. This effect is the greatest in the small amplitude case of $5^{\circ}$. At a constant reduced frequency, the phase lag increases slightly with freestream velocity. In Table 4.2 below a summary of rms and phase data is presented.

TABLE 4.2

Freestream Response due to Model Motion

\begin{tabular}{|c|c|c|c|c|}
$\begin{array}{c}\text { amplitude } \\
\text { (deg) }\end{array}$ & $\begin{array}{c}\text { Reynolds } \\
\text { number }\end{array}$ & $\mathrm{k}$ & $\begin{array}{c}\text { vel. fluctuation } \\
\% \mathrm{rms}\end{array}$ & $\begin{array}{c}\text { phase lag } \\
\text { (deg) }\end{array}$ \\
5.0 & 260,000 & 0.05 & 0.457 & 13.4 \\
5.0 & 260,000 & 0.30 & 0.332 & 50.1 \\
& & & & \\
5.0 & 90,000 & 0.20 & 0.312 & 17.2 \\
5.0 & 350,000 & 0.20 & 0.373 & 25.0 \\
22.5 & 260,000 & 0.05 & 1.177 & 5.7 \\
22.5 & 260,000 & 0.30 & 0.729 & 32.5
\end{tabular}


To provide a reference for the unsteady cases, the static vortex position was recorded for both increasing and decreasing angle of attack. The angle of attack range was $29^{\circ}$ to $39^{\circ}$, the same as for the small amplitude dynamic cases. Statistical information on the fluctuation of breakdown position was also obtained. A DFT was performed on the vortex breakdown position at the minimum and maximum angles of attack, and the rms of the breakdown location was computed at each incremental angle of attack.

At each angle of attack recorded, an average breakdown location was computed using 10 equally spaced movie frames which spanned approximately 1.5 seconds of data. Static breakdown location was plotted as a function of angle of attack for both increasing and decreasing angle of attack, and the results are presented in Figure 4.4. The curves compare nicely at angles of attack above $31^{\circ}$, but below this value they deviate indicating a possible static hysteresis in this range of angle of attack. In this range, the breakdown location is farther downstream in the increasing static case than it is in the decreasing static case. Lowson (1964) observed a similar phenomenon on an $80^{\circ}$ sweep delta wing, in which it took a lower static angle of attack to move the breakdown position into the wake of the model than it did to move it onto the trailing edge.

At a fixed angle of attack, the vortex breakdown location is not stationary, but rather oscillates or moves back and forth with no apparent dominate frequency. To verify this, DFTs were performed on the position of breakdown location. This was done at approximately $29^{\circ}$ and $39^{\circ}$, the extreme values of angle of attack in the static tests. At each angle of attack, 128 data points were taken at a frequency of $64 \mathrm{~Hz}$ based on film speed, and the resolution was \pm 0.5 $\mathrm{Hz}$. The DFT results are presented in Figures $4.5 \mathrm{a}$ and $\mathrm{b}$ where frequency is 
plotted as a function of amplitude. There is no apparent dominate frequency or harmonics in either case, which indicates a random oscillation of breakdown position over the frequency range of 0 to $32 \mathrm{~Hz}$. The largest amplitude occurred at $1 \mathrm{~Hz}$ in each case, and as frequency increased the amplitude decreased on the average.

The amount of oscillation was also investigated on the static data for both increasing and decreasing angle of attack. The rms of the breakdown position was computed using the 10 data points at each angle of attack. The rms data provided a measure of the average oscillation in breakdown location. A bar graph in Figure 4.6 shows the relative rms at each angle of attack. No noticeable trend is evident from the rms data. The average rms for the entire angle of attack range is $0.013 \times / \mathrm{c}$.

\subsection{Vortex Breakdown Dynamics using High Speed Cinema}

This section addresses the dynamic response of vortex breakdown due to dynamic motion. Data presented includes; chordwise breakdown location, phase information between model motion and breakdown location, propagation velocity of breakdown location, and vortex core inclination angle. The data is the result of digitized high speed motion pictures which have been ensemble averaged over several cycles of oscillation as explained in Section 3.6. Spanwise breakdown position data, acquired using $35 \mathrm{~mm}$ photography, will be discussed in a following section. 


\subsubsection{General Description of Vortex Flow Response}

Before detailed analysis is presented, a general description of vortex response on a delta wing due to sinusoidally pitching will be given. The flowfield development is very similar to that on a static delta wing. As the angle of attack increases from $0^{\circ}$, the flow transitions from attached flow to separated flow, in which two counter rotating vortices are formed above the upper surface of the wing. As the angle of attack continues to increase, the position of vortex breakdown occurs first in the wake and then travels upstream across the trailing edge towards the model apex. As angle of attack decreases, the breakdown position moves downstream, recrosses the trailing edge, and eventually into the wake. The vortex flow then reattaches to the model surface with decreasing angle of attack.

Most of the data in this study focuses on an angle of attack range for which vortex breakdown location remains over the wing, namely $29^{\circ}$ to $39^{\circ}$. A sequence of photographs produced from $16 \mathrm{~mm}$ motion picture film for this angle of attack range is shown in Figures 4.7(a-j). The photographs illustrate how the breakdown location varies with angle of attack throughout a pitching cycle. It is evident from the photographs that there is a lag in the breakdown location relative to the model position as the model oscillates. The location of vortex breakdown is further downstream on the upstroke than it is on the downstroke, at the same angles of attack. This lag presents itself in the form of a hysteresis loop when the breakdown location is plotted against angle of attack for a pitching cycle, and quantified results are presented in the next section. Photographs for the large amplitude case of $0^{\circ}$ to $45^{\circ}$, in which separation and reattachment of the vortex flow occurs, will be presented and discussed in a later section. 


\subsection{2 x/c vs Angle of Attack}

Four cases were investigated to determine the effect of reduced frequency on chordwise breakdown location over the angle of attack range of $29^{\circ}$ to $39^{\circ}$ degrees at a constant freestream velocity of $30 \mathrm{ft} / \mathrm{s}$ corresponding to a root chord Reynolds number of 260,000 . The cases are summarized below.

TABLE 4.3

Constant Reynolds Number Cases ( $\alpha=29^{\circ}$ to $39^{\circ}$ )

\begin{tabular}{|c|c|c|c|}
$\begin{array}{c}\text { Reynolds } \\
\text { number }\end{array}$ & $k$ & $\begin{array}{c}\text { \# cycles } \\
\text { averaged }\end{array}$ & $\begin{array}{c}\text { pitching } \\
\text { trequency(Hz) }\end{array}$ \\
260,000 & 0.05 & 8 & \\
260,000 & 0.10 & 8 & 0.174 \\
260,000 & 0.20 & 20 & 0.347 \\
260,000 & 0.30 & 9 & 0.694 \\
& & 1.042
\end{tabular}

Nondimensional chordwise breakdown location is plotted as a function of angle of attack in Figures 4.8 (a-d) for a pitching cycle, along with static data obtained over the same angle of attack range and freestream velocity. The static data is an average of the increasing and decreasing static angle of attack cases discussed in Section 4.4. Therefore the previously observed static hysteresis at the lower angle of attack range has been averaged out.

At the lowest value of reduced frequency where $k=0.05$, a hysteresis loop develops in the location of vortex breakdown. As the reduced frequency increases the hysteresis effect becomes greater, at a given angle of attack, and the $x / C$ range over which breakdown occurs decreases. A hysteresis in breakdown location was also observed by Rockwell et al (1987). They 
observed the development of hysteresis loops at reduced frequencies as low as 0.16 on a $45^{\circ}$ sweep delta wing. Gad-el-Hak and Ho (1985) observed similar hysteresis loops as well, but not in breakdown location. They observed a hysteresis loop in the growth and decay in the height of the vortex above the wing at a specific $x / c$ location.

The decrease in the range of $x / c$ is more apparent at the low values of angle of attack where the breakdown position reaches its maximum $x / c$ value. In this region, the maximum $x / c$ value decreases from approximately $0.925 x / C$ to $0.825 \mathrm{x} / \mathrm{c}$, with increasing reduced frequency. At the higher reduced frequencies, the wing starts to pitch-up again before the breakdown position can reach its aft most static location.

At the lowest reduced frequency of $k=0.05$, an overshoot in the burst position past the static case is evident. This overshoot is slight and occurs at the minimum angle of attack. At the higher reduced frequencies, no overshoot is evident in the hysteresis loops. Atta and Rockwell (1987) observed an overshoot in breakdown location relative to the static case as well.

The resulting hysteresis loops become more symmetric about the static case as reduced frequency is increased. At reduced frequencies of $k=0.05$ and 0.10 , the breakdown position lags the static case on the upstroke and follows it on the downstroke. As reduced frequency is further increased to $k=$ 0.20 , the amount by which the breakdown location lags the static case on the upstroke increases, and a similar lag began to develop on the downstroke side. At a reduced frequency of $k=0.30$, the hysteresis loop appears to reach a symmetric state about the static data, with the lag in breakdown position being approximately equal for both upstroke and downstroke phases of the pitching cycle. 
Four additional cases were investigated to determine the effect of Reynolds number on chordwise breakdown location over the same angle of attack range of $29^{\circ}$ to $39^{\circ}$. The reduced frequency was held constant at $k=0.20$. The test conditions are summarized in Table 4.4 below.

TABLE 4.4 Constant Reduced Frequency Cases $\left(\alpha=29^{\circ}\right.$ to $\left.39^{\circ}\right)$

\begin{tabular}{|c|c|c|c|}
$\mathrm{Re}$ & $\mathrm{k}$ & $\begin{array}{c}\text { \#cycles } \\
\text { averaged }\end{array}$ & $\begin{array}{c}\text { pitching } \\
\text { frequency }(\mathrm{Hz})\end{array}$ \\
90,000 & 0.20 & 9 & 0.232 \\
175,000 & 0.20 & 12 & 0.463 \\
260,000 & 0.20 & 20 & 0.694 \\
350,000 & 0.20 & 8 & 0.926
\end{tabular}

The results from the above tests are presented in Figures 4.9a-c. In Figure $4.9 a$ the results from the two lower Reynolds number cases are presented together, and in Figure $4.9 \mathrm{~b}$ the two higher Reynolds number cases are presented together. In Figure 4.9c, all four cases are presented together. There is little difference between the two plots in Figure 4.9a and the two plots in Figure 4.9b, indicating no evident Reynolds number effects occur between $R e$ $=90,000$ and 175,000 , and between $\operatorname{Re}=260,000$ and 350,000 .

However, when all four cases are plotted together there is a significant difference between the two lower and two higher Reynolds number cases, indicating a change in the dynamic behavior of vortex breakdown between Reynolds numbers of $R_{\theta}=175,000$ and 260,000 . The vortex breakdown location at the lower Reynolds numbers is more forward on the wing than at the 
higher Reynolds numbers. This shift is observable over the entire pitching cycle with its effect being greatest at the lower angles of attack. Gad-el-Hak and Ho (1985) observed no significant change in the resulting hysteresis loops for tests conducted at Reynolds numbers of 25,000 and 340,000 at a constant reduced frequency of 2.0. However, as previously noted, the hysteresis loop observed in their experiment was based on the height of the vortex core at a specific $x / C$ location and not on breakdown position. Also, their angle of attack range was $0^{\circ}$ to $30^{\circ}$.

It is interesting to note that in the four cases presented in this study, the size of the hysteresis loop does not change significantly, but rather just shifts to a new position. No explanation for the phenomena has been determined.

\subsubsection{Width of the Hysteresis Loop}

The width of the hysteresis loops at specific angles of attack were computed using the breakdown location vs angle of attack plots presented in Figures $4.8 \mathrm{a}-\mathrm{d}$ and $4.9 \mathrm{a}-\mathrm{c}$. The width was determined to quantify the changes in vortex breakdown location between the upstroke and downstroke phases of the pitching cycle. The data was obtained through graphical means by measuring the distance between the upstroke and downstroke sides of the hysteresis loops at the specific angles of attack of $30^{\circ}, 32^{\circ}, 34^{\circ}, 36^{\circ}$ and $38^{\circ}$. It should be noted that the data came from interpolation of the curves and not from actual data points. Straight lines were drawn between the data points in the hysteresis loops, and used to represent the location of vortex breakdown. However, because the data points are closely spaced, the straight lines provide a good estimate of the position of vortex breakdown. 
In Figures 4.10a and b, nondimensional width of the hysteresis loop is plotted as a function of angle of attack in units of $x / c$ for constant Reynolds number and varying reduced frequency, and for constant reduced frequency and varying Reynolds number respectively. In Figure $4.10 \mathrm{a}$ the width of the hysteresis loop increases with reduced frequency. At $k=0.05$ and 0.10 the maximum width is reached at the minimum angle of attack of $30^{\circ}$ and then steadily decreases as angle of attack increases. A variation in this behavior occurs as reduced frequency is further increased. At $k=0.20$ the maximum width is attained at $\alpha=32^{\circ}$, increasing from $30^{\circ}$, where it drops off steadily through $\alpha=38^{\circ}$. At $k=0.30$, the width reaches a maximum value at $\alpha=32^{\circ}$ and remains at that approximate value through $\alpha=36^{\circ}$, and then drops off sharply. In general, maximum width is reached at higher angles of attack with increasing reduced frequency.

In Figure 4.10b, the results for a constant reduced frequency and varying Reynolds are presented. Each curve, corresponding to a specific Reynolds number, is similar in shape with the width increasing relatively rapidly from $\alpha=$ $30^{\circ}$ to $\alpha=32^{\circ}$, where a maximum is reached. The width then decreases with increasing angle of attack, with the rate of decrease becoming greater as angle of attack increases. Overall, the variation in width is relatively small between cases, with the greatest variation occurring at the minimum angle of attack. The variation was almost nonexistent at the maximum angle of attack. In general, width increases slightly with Reynolds number, except for the two highest cases in which the average width at a $R \theta=260,000$ is greater than at a $R e=350,000$. 
4.5.4 Phase Difference between Breakdown Location and Model Position

Phase angle difference between chordwise breakdown location and model position was obtained for the dynamic cases above in which the Reynolds number was constant at 260,000 and the reduced frequency varied between $k=0.05$ and 0.30 , and for the reduced frequency being constant at $k=$ 0.20 and Reynolds number varied between $R \theta=90,000$ and 350,000 . A value for the phase lag was obtained by plotting breakdown location and model angular position together against time for a cycle of motion. A sample plot is shown in Figure 4.11. The phase angle was calculated, through graphical means, by determining the time between maximum and minimum amplitudes. The time difference was determined between the maximum angle of attack and minimum breakdown location, as well as between the minimum angle of attack and maximum breakdown location. The maximum and minimum amplitudes were determined visually, and the distances between them along the time axis measured. The distances were converted to a corresponding time, and then divided by the period and multiplied by $360^{\circ}$ to determine the phase differences. The two phase differences were averaged to obtain a value for the phase lag. The plots used to provide the information can be seen in Appendix C.

In Figure 4.12, the phase lag is plotted as a function of reduced frequency for a constant Reynolds number of 260,000 . It is evident that a near linear relationship exists between phase lag and reduced frequency. As the reduced frequency increases, so does the phase lag. The maximum phase lag is approximately $22^{\circ}$ at the maximum reduced frequency of $k=0.30$ The increase in phase lag occurs because the vortex flow becomes less able to keep up with the motion of the model at the higher reduced frequencies. 
Maltby et al (1963) observed a phase lag in vortex flow for a delta wing of aspect ratio one oscillating in heave at an incidence of $22^{\circ}$. They found a phase lag of $60^{\circ}$ at a reduced frequency of 3.4 and $51^{\circ}$ at a reduced frequency of 1.13 . They assumed that a straight line could be drawn through the vortex core up to approximately $0.7 \mathrm{x} / \mathrm{c}$. The height of the vortex core was quantified at several $x / c$ locations along the model and it was found that a near linear relationship existed up to $0.7 \mathrm{x} / \mathrm{c}$. The phase lag was computed by comparing the slope of the vortex core to the model motion at various locations throughout an oscillation cycle.

Figure 4.13 illustrates the effect of Reynolds number on phase lag at a constant reduced frequency of $k=0.20$. The phase lag remains relatively constant for Reynolds numbers between 90,000 and 350,000 . The maximum variation is $2.6^{\circ}$, from a value of $14.6^{\circ}$ at a Reynolds number of 90,000 to a value of $17 \cdot 2^{\circ}$ at a Reynolds number of 350,000 . Therefore, a change in Reynolds number appears to have little effect on phase lag over the range investigated.

It is important to realize that it is a change in the reduced frequency and not the pitching frequency which affects phase lag. This point is illustrated in Figure 4.14 where phase lag is plotted against pitching frequency for both constant reduced frequency and constant Reynolds number. Both curves span approximately the same range of pitching frequencies, yet are very different. The constant Reynolds number curve increases linearly with pitching frequency, while the constant reduced frequency curve increases only slightly. 
4.5.5 Propagation Velocity of Breakdown Location

The propagation velocity of breakdown location relative to delta wing was computed over a cycle of motion for each of the small amplitude cases ( $\alpha=$ $29^{\circ}$ to $3^{\circ}$ ) presented above. Velocity was obtained by dividing the incremental $x / c$ distance between two consecutive breakdown locations by the corresponding incremental time which was obtained by knowing the number of movie frames between data points and the film rate. Central differencing was used for the calculation, meaning that the value of the velocity was computed at the average $x / c$ value of the data points used in the computation. A positive velocity corresponded to the breakdown location moving downstream, and a negative velocity corresponded to the breakdown position moving upstream. The process was repeated from data point to data point throughout the entire pitching cycle.

In Figures 4.15 and 4.16 propagation velocity (ft/s) was plotted against angle of attack for varying reduced frequency and a constant Reynolds number of $R e=260,000$, and for varying Reynolds number and a constant reduced frequency of $k=0.20$, respectively. In Figure 4.15 , the magnitude of the velocity increases with reduced frequency, with the maximum and minimum velocities being approximately equal for each case. The changes in velocity are more sudden at the lower angles of attack, when the burst is closer to the trailing edge, than at the higher angles of attack, when the burst is closer to the apex. Maximum velocity is attained in the angle of attack range of $30^{\circ}$ to $32^{\circ}$ on the downstroke, and minimum velocity is attained in the angle of attack range of $31^{\circ}$ to $33^{\circ}$ on the upstroke. The maximum propagation velocity was $1.6 \mathrm{ft} / \mathrm{s}$ at a reduced frequency of $k=0.30$. 
In Figure 4.16 where Reynolds number is varied for constant reduced frequency of $k=0.20$, the magnitude of the velocity increases with Reynolds number. Again, the maximum and minimum values of velocity are approximately equal for each of the cases, and the curves follow the same trends as the varying reduced frequency cases. A maximum velocity of $1.6 \mathrm{ft} / \mathrm{s}$ is attained at the highest Reynolds number of 350,000 , which is approximately equal to $1 / 25$ of the freestream velocity. The maximum velocities obtained in the other cases are also approximately equal to $1 / 25$ of the corresponding freestream velocities.

The increase in velocity observed in the two cases seems more dependent on pitching frequency than reduced frequency or Reynolds number. This is thought to be because the range of pitching frequencies spanned is approximately equal in both cases, and the corresponding velocity plots are very similar. In other words, as the model is pitched with increasing angular velocity, the speed of breakdown propagation also increases. To eliminate this effect, a reduced velocity $(\mu)$ is defined where $\mu=u /(c f)$. The curves in Figures 4.15 and 4.16 are replotted using reduced velocity, and are shown in Figures 4.17 and 4.18. From the plots, the effects of reduced frequency and Reynolds number can be seen.

The effect of reduced frequency is minimal throughout most of the pitching cycle as seen in Figure 4.17, and the maximum reduced velocity in each case is approximately 1.2 which occurs in the angle of attack range of $30^{\circ}$ to $33^{\circ}$. The curves of varying reduced frequency collapse upon themselves, except in the region at the beginning of the upstroke where angle of attack starts increasing. More specifically, the change takes place between $\alpha=29^{\circ}$ and $\alpha=$ $35^{\circ}$. In this region the reduced velocity becomes more negative with decreasing reduced frequency. Therefore, the reduced velocity with which the 
burst moved up the model increases. This increase is related to the shape of the hysteresis loops as illustrated previously in Figures 4.8a-d. Over the range of angle of attack in which the change occurs, the location of vortex breakdown is further downstream in the lower reduced frequencies cases, and the distance between data points is greater. This greater distance results in a higher value of reduced velocity.

The effect of Reynolds number on reduced velocity can be seen in Flgure 4.18. No distinct changes are apparent between the four cases with the reduced velocities being very similar. The maximum reduced velocity is approximately 1.2 in each case, which occurs between $30^{\circ}$ and $33^{\circ}$. Therefore, a change in Reynolds number has little effect on the reduced propagation velocity of breakdown location over the Reynolds number range of 90,000 to 350,000 , at a reduced frequency of $k=0.20$.

\subsubsection{Oscillation of Breakdown Position}

As the breakdown location moves up and down the model with increasing and decreasing angle of attack, it oscillates back and forth in an irregular fashion, similar to the manner with which it behaves in the static case as described in Section 4.3. Therefore, the $x / c$ location of vortex breakdown differs from cycle to cycle at corresponding angles of attack due to this random oscillation. The amount by which the breakdown position varies at specific angles of attack, throughout the pitching cycle, was quantified by computing the rms of the burst fluctuation as in Section 4.3 for static data.

In Figures 4.19a and $b$, the rms of the burst fluctuation is plotted for two cases at specific angles of attack throughout the cycle. These cases were selected because they contain the greatest number of cycles, and the larger the 
number of cycles the more representative the rms value. In Figure 4.19a, the rms is computed from 20 cycles in which $R e=260,000$ and $k=0.20$, and in Figure 4.19b, the rms is computed from 12 cycles in which $R e=175,000$ and $k=$ 0.20. No apparent trend was observed in the rms distribution in either case. An average rms of breakdown location was computed for the entire pitching cycle. The resulting average $\mathrm{rms}$ is $0.020 \mathrm{x} / \mathrm{c}$ for the first case and $0.019 \times / c$ for the second. In addition, the average rms was computed for the rest of the dynamic cases in which the angle of attack range was $29^{\circ}$ to $39^{\circ}$. The results are summarized in Table 4.5 , and the resulting rms values are fairly consistent.

TABLE 4.5

Average rms of Breakdown Location

\begin{tabular}{|c|c|c|c|}
$R \theta$ & $k$ & $\begin{array}{c}\text { \#cycles } \\
\text { averaged }\end{array}$ & $\begin{array}{c}\text { average } \\
\text { rms }(x / c)\end{array}$ \\
260,000 & 0.05 & 8 & 0.019 \\
260,000 & 0.10 & 8 & 0.017 \\
260,000 & 0.20 & 20 & 0.019 \\
260,000 & 0.30 & 9 & 0.018 \\
& & & \\
90,000 & 0.20 & 9 & 0.050 \\
175,000 & 0.20 & 12 & 0.020 \\
260,000 & 0.20 & 20 & 0.019 \\
350,000 & 0.20 & 8 & 0.022
\end{tabular}

The overall weighted average of the rms of breakdown location for the 8 dynamic cases is $0.022 \times / c$. This is a $70 \%$ increase over the average rms value of $0.013 \times / c$ obtained in the static case over the same angle of attack range. 


\subsubsection{Vortex Core Inclination Angle}

To provide additional information on the response of vortex breakdown due to pitching motion, a vortex core inclination angle $(\beta)$ was defined. This was the angle between the model surface and a straight line connecting the model apex and breakdown location. The line was used to represent the vortex core, which appeared to be relatively straight throughout the $29^{\circ}$ to $39^{\circ}$ angle of attack range. The validity of this observation was proven by conducting a simple visual experiment. A straight edge was lined up with the model apex and breakdown location on projected images from the $16 \mathrm{~mm}$ motion picture film. The observation was performed at various angles of attack throughout the pitching cycle. The vortex core was found to track the straight edge relatively well at all angles of attack investigated. To illustrate this, two photographs are shown in Figures $4.20 \mathrm{a}$ and $\mathrm{b}$ with superimposed lines connecting the model apex and breakdown location. From these photographs it can be seen how closely the vortex core follows a straight line. The photographs represent positions in the pitching cycle at which approximately the largest and smallest $x / c$ breakdown locations are encountered for the $29^{\circ}$ to $39^{\circ}$ angle of attack range.

The results for varying reduced frequency and constant Reynolds number are shown in Figures 4.21 and 4.22, and the results for varying Reynolds number and constant reduced frequency are shown in Figures 4.23 and 4.24. These plots provide an indication of the amount the vortex core moves up and down in relation to the model surface over a cycle of oscillation. In the constant Reynolds number plots, $\beta$ varies from approximately $8.2^{\circ}$ at the lowest angle of attack, to $10.1^{\circ}$ at the highest angle of attack. At the lowest reduced frequencies of $k=0.05$ and $k=0.10$, no significant changes occur 
between the upstroke and downstroke phases of the cycle, i.e., no hysteresis loop is formed. However, at a reduced frequency of $k=0.20$, a change occurs between the upstroke and downstroke phases of the cycle and a hysteresis loop is formed. The change occurs between $\alpha=32^{\circ}$ and $\alpha=39^{\circ}$, with no obvious change between $29^{\circ}$ and $32^{\circ}$. At $k=0.30$, a hysteresis is evident over the entire range of angle of attack, with the hysteretic effect being the greatest at the higher angles of attack.

The range of $\beta$ for the constant reduced frequency cases varies from approximately $7.9^{\circ}$ to $9.7^{\circ}$ in the lowest Reynolds number case of $R \theta=90,000$, and from approximately $8.2^{\circ}$ to $10.0^{\circ}$ in the higher Reynolds number cases of $R e=175,000,260,000$ and 350,000 . At a Reynolds number of $R e=90,000$ no distinct hysteresis in $\beta$ is apparent. At the higher Reynolds numbers of $R e=175,000,260,000$ and 350,000 , a hysteresis occurs which is more apparent at the higher values of angle of attack. Overall, a change in Reynolds number appears to have little effect on the vortex core inclination angle. However, a slight shift of approximately $0.3^{\circ}$ in the range of $\beta$ did seem occur between Reynolds numbers of 90,000 and 175,000 .

\subsubsection{Large Amplitude Oscillations}

Up to now, all oscillatory data has come from a relatively small range of angle of attack in which the wing was pitched from $29^{\circ}$ to $39^{\circ}$. Data for the large amplitude motion in which the wing was pitched from $0^{\circ}$ to $45^{\circ}$ is presented in this section. Reduced frequencies of $k=0.05$ and 0.30 were investigated at a root chord Reynolds number of 260,000 . The data is presented in much the same way as for the small amplitude case, with chordwise breakdown location, phase lag, and vortex inclination angle discussed. However, the data is not 
representative of the entire pitching cycle due to the breakdown location moving into the wake of the model as angle of attack decreases. As the breakdown location moves into the wake, it leaves the camera's field of view and cannot be measured. Therefore, data from only a part of the pitching cycle will be presented. Because of this limitation, information pertaining to the width of the hysteresis loop, the velocity of breakdown propagation, and the fluctuation of the breakdown location are not included.

The vortex response for a complete pitching cycle is represented in Figures $4.25 \mathrm{a}-\mathrm{j}$ by a sequential set of photographs produced from $16 \mathrm{~mm}$ motion picture film, in which the reduced frequency is 0.30 and the Reynolds number is 260,000 . A large hysteresis in the vortex flow is clearly visible when the upstroke and downstroke phases are compared at the same angles of attack. A hysteresis was also observed in the $k=0.05$ case. Both cases will be compared in data which follow.

The vortex flow separation and reattachment processes can be seen in a second set of photographs shown in Figures 4.26a-j. The term "flow separation" is defined as the existence of separated vortical flow over the entire surface of the wing. Figures $4.26 a-\theta$ illustrate flow separation, and Figures $4.26 f-j$ illustrate flow reattachment. The photographs in each set were produced from consecutive $16 \mathrm{~mm}$ movie frames which were separated by $1 / 64 \mathrm{sec}$. From the photographs, the reattachment and separation processes appear very different. As the angle of attack increases, some of the flow seems to separate all along the leading edge while a majority of the flow stays attached (illustrated by tracer coming off the trailing edge). As the angle of attack continues to increase, the flow transitions to completely separated flow. During flow reattachment, the flow seems to first attach at the apex and then as angle of attack decreases, the point of reattachment moves toward the trailing edge until the flow is completely 
attached. As the angle of attack decreases, a condition does not occur in which the flow is both separated and attached, as in the increasing angle of attack case.

The angles of attack at which separation and reattachment start to occur were recorded for the reduced frequencies of $k=0.05$ and 0.30 . In the $k=0.05$ case, flow separation starts at an angle of attack of $11^{\circ}$, and is completely separated at an angle of attack of $18^{\circ}$. Reattachment starts at $\alpha=13^{\circ}$ and is complete at $\alpha=8^{\circ}$. The difference between the average incidence angles of separation and reattachment is $4^{\circ}$. In the $k=0.30$ case, the flow starts to separate at $\alpha=15^{\circ}$ and is complete at $\alpha=23^{\circ}$. Reattachment starts at $\alpha=6^{\circ}$ and is complete at $4^{\circ}$. The difference between the average incidence angles of separation and reattachment in this case is $14^{\circ}$ as compared to $4^{\circ}$ in the $\mathrm{K}=$ 0.05 case. Therefore, the effect of hysteresis, as observed in the separation and reattachment processes, is much greater in the $k=0.30$ case which was expected.

The vortex breakdown location was plotted as a function of angle of attack in Figure $4.27 \mathrm{a}$ for both reduced frequencies of $k=0.05$ and 0.30 at a Reynolds number of 260,000 . A hysteresis is evident in both cases, with the effect being much greater in the $k=0.30$ case. On the upstroke, the position of vortex breakdown is approximately the same for both cases at angles of attack greater than $32^{\circ}$. On the downstroke however, a large variation occurs in the position of vortex breakdown. The breakdown location for the $k=0.30$ case greatly lags the breakdown location of the $k=0.05$ case, with the variation increasing with decreasing angle of attack. Therefore, a majority of the hysteresis occurs during the downstroke phase over this range of attack. The maximum hysteresis in breakdown location (while over the wing) occurs at approximately $30^{\circ}$ for the $k=0.30$ case and $32^{\circ}$ for the $k=0.05$ case. 
In Flgure 4.27b, the breakdown location for the large amplitude case is compared to the breakdown location for the small amplitude case of $29^{\circ}$ to $39^{\circ}$ at the same reduced frequencies of $k=0.30$ and Reynolds numbers of $R e=$ 260,000 . Also presented is static data which was obtained for the small amplitude case. At any given angle of attack, the hysteresis effect is much greater for the large amplitude case. Again, the large change occurs in the downstroke phase of the cycle, and the static data lies much closer to the upstroke side of the large amplitude case. This increased hysteretic effect is possibly a result of the increased rate at which the angle of attack is changing.

A phase lag for the vortex flow was computed through graphical means as for the small amplitude cases. Vortex breakdown location and model angular position were plotted as a function of time and the horizontal distance between the maximum angle of attack and minimum breakdown location was measured to provide the phase information. Minimum angle of attack could not be used as in the small amplitude case, because at that position the flow was attached and vortex breakdown did not occur. The plots used to obtain the information can be found in Appendix $C$.

The phase lag in the $k=0.05$ case is $5^{\circ}$ and the phase lag in the $k=0.30$ case is $20^{\circ}$. These values are very similar to the phase lags obtained in the $29^{\circ}$ to $39^{\circ}$ cases for the same reduced frequencies and freestream Reynolds number. The values obtained in the small amplitude cases were $5^{\circ}$ and $22^{\circ}$ for reduced frequencies of $k=0.05$ and $k=0.30$ respectively.

The vortex core inclination angle (relative to the model) was plotted against angle of attack for the segments of the pitching cycle for which vortex breakdown occurred over the wing. Both reduced frequencies were plotted together and shown in Flgure 4.28. The angle was computed as in section 4.5.7 in which a straight line was drawn from the model apex to breakdown 
location to represent the vortex core. The vortex core inclination angle attained a maximum value of $12^{\circ}$ in the $k=0.05$ case and $13.5^{\circ}$ in the $k=0.30$ case. A hysteresis in the flow was again evident with the hysteretic effect being greater for the higher value of reduced frequency with the greatest change occurring on the downstroke, as like the plots representing vortex breakdown location.

\subsubsection{Impulsive Change in Angle of Attack}

It was felt that it would be important to include a few results from early investigative work performed, in order to illustrate some trends which were observed for an impulsive increase and impulsive decrease in angle of attack. The model was the same as the one used in the previously described experiments, except for the trailing edge which was not beveled. The wing was impulsively pitched from $28^{\circ}$ to $37^{\circ}$ (the maximum and minimum amplitude of the motion), and also from $37^{\circ}$ to $28^{\circ}$. The wing was pitched as if it were being sinusoidally oscillated, but only for half the cycle. For the pulse-up, motion was initiated at minimum amplitude and stopped at maximum amplitude, and for the pulse-down, the opposite procedure was performed.

The model angular position and vortex breakdown location was plotted against reduced time for pulse-up and pulse-down, and the data is shown in Figures 4.29 and $b$ respectively. This data is the result of 3 data records which were ensemble averaged. The model was pitched at a rate corresponding to a reduced trequency of $k=0.45$ (based on a complete cycle of motion). Even though the impulsive pitch was part of a sinusoidal motion, the pitching rate was fairly constant which can be observed in the plots. The approximate value of the pitch rate was $0.7 \mathrm{rad} / \mathrm{s}$ corresponding to a 
nondimensional pitch rate of $K=0.016$, and the freestream Reynolds number was 350,000 .

In both cases the vortex response is immediate with model motion, but some time is required for the breakdown position to reach a steady state once the motion ceases. These recovery times range from 4 to 7 convective times units $(\tau)$ for both the pitch-up and pitch-down cases. During the motion, the convective time lag was approximately 1 unit. Lambourne et al (1969) also observed a delay in the vortex flow to steady state, in a plunging delta wing experiment, after the plunging motion had ended. Reynolds and Abtahi (1987) performed an experiment in which a $75^{\circ}$ sweep delta wing was impulsively pitched at a constant rate of $K=0.06$ from $30^{\circ}$ to $51^{\circ}$ and from $51^{\circ}$ to $30^{\circ}$, at chord Reynolds numbers between 19,000 to 65,000 . They observed long nondimensional response times in the location of vortex breakdown to reach a steady state after motion had ceased, and reported a hysteresis in the pitch-up and pitch-down cases which was made evident by the differences in the initial movement of vortex breakdown. In this study however, no significant hysteresis was apparent.

\subsection{Vortex Breakdown Dynamics using 35mm Photography}

To provide information on the spanwise location of vortex breakdown, $35 \mathrm{~mm}$ photographs were taken at three reduced frequencies of $k=0.05,0.20$ and 0.30 at a Reynolds number of 350,000 at 4 location within the cycle as described in Section 3.4. It was hoped that the photographs taken at the mean angles of attack on upstroke and downstroke would provide information on the amount of hysteresis in the vortex flow in the spanwise position. This was not the case however, because a time lag in the data acquisition system was not 
accounted for. This resulted in a phase shift of the recorded angular positions, which increased with increasing pitching frequency. Therefore, no conclusive determination of hysteresis could be made.

Because of this problem, no ensemble averaging of the acquired data or error analysis was performed. However, the data is presented and shown in Figures 4.30 , where the spanwise position of vortex breakdown is plotted against angle of attack for the varying reduced frequency. At each data point, a set of photographs was digitized which was most representative of the 5 sets available. The selection was made by taking a visual average of the available photographs. A few observations were made from the data.

Throughout the angle of attack range of $29^{\circ}$ to $39^{\circ}$ the variation in the spanwise breakdown location was very small, being approximately $3 \% \mathrm{y} / \mathrm{s}$. The values ranged from approximately $0.58 \mathrm{y} / \mathrm{s}$ to $0.61 \mathrm{y} / \mathrm{s}$, with the spanwise location moving outboard on the average as the angle of attack increased. It is important to realize however that this data is the result of a limited amount of data, and therefore, should be used only as an indicator of possible trends. To obtain a better representation of the spanwise motion of vortex breakdown during sinusoidal oscillations, further investigation is necessary. 


\section{CHAPTER V}

CONCLUSIONS AND RECOMMENDATIONS

The major objective of this study was to investigate the dynamic response of the vortex flow over a sharp-edged, $70^{\circ}$ flat plate delta wing, while undergoing unsteady motions. A $70^{\circ}$ delta wing was sinusoidally oscillated about its midchord position at reduced frequencies ranging from $k=0.05$ to 0.30 over the angle of attack ranges of $29^{\circ}$ to $39^{\circ}$, and $0^{\circ}$ to $45^{\circ}$, at freestream Reynolds numbers between 90,000 and 350,000 based on root chord. The wing also impulsively pitched from minimum to maximum amplitude, and from maximum to minimum amplitude, at a Reynolds number of 260,000 over the angle of attack range of $28^{\circ}$ to $37^{\circ}$.

Towards this end, an experimental facility was designed and fabricated which was capable of sinusoidally or impulsively pitching a delta wing model throughout a wide range of pitching frequencies and amplitudes. In addition, a flow visualization technique using titanium tetrachloride was developed which provided continuous quality flow visualization of the vortex flow and breakdown phenomenon during pitching motion.

The major conclusions from the experiment are presented below, and suggested recommendations for future research follow. 


\subsection{Conclusions}

As the delta wing was oscillated in a sinusoidal fashion in the angle of attack range of $29^{\circ}$ to $39^{\circ}$ at reduced frequencies of $k=0.05$ to $k=0.30$, a hysteresis developed in the chordwise position of vortex breakdown which manifested itself in the form of a elongated hysteresis loop when breakdown location was plotted against angle of attack. A hysteresis is observed in the chordwise position of vortex breakdown at a reduced trequency as low as $k=$ 0.05 . As the reduced frequency is further increased, the hysteresis effect becomes greater. At a given angle of attack, the distance between the position of vortex breakdown on the upstroke and the downstroke increases, and the maximum $x / c$ position attained by vortex breakdown during the motion decreases. At the lower reduced frequencies, the hysteresis in breakdown location is greatest at the lowest angle of attack within the cycle, and at the higher reduced frequencies, the hysteresis effect is greatest at higher angles of attack.

When the Reynolds number is varied between 90,000 and 350,000 for a fixed reduced frequency of $k=0.20$ a significant change occurs in the response of the vortex flow between Reynolds numbers of 175,000 and 260,000 . The location of chordwise vortex breakdown shifts to a more downstream position on the wing for an increase in Reynolds number at the same angle of attack. The shift takes place over the entire pitching cycle, with the shift being the greatest at lower angles of attack.

The phase lag in the position of vortex breakdown relative to the angular position of the wing was also calculated. The phase lag increases linearly with reduced frequency, and the maximum phase lag is approximately $22^{\circ}$ at a reduced frequency of $k=0.30$. The freesteam Reynolds number was 260,000 . 
A reduced propagation velocity $\{u /(c f)\}$ of breakdown location was computed for the varying reduced frequency and varying Reynolds number cases as well. In every case, the maximum reduced velocity obtained is approximately equal to \pm 1.2 . This occurs over the angle of attack range of $30^{\circ}$ to $33^{\circ}$. A change in Reynolds number was found to have little effect on the reduced velocity. A change in reduced frequency also has little effect, except in the part of the pitching cycle where angle of attack starts to increase after the model passes through minimum amplitude. Here the magnitude of the reduced velocity increases with decreasing reduced frequency.

The average rms of the breakdown location, obtained at discrete angles of attack throughout the pitching cycle for all cases investigated, was found to be $0.022 \times / c$. This is $70 \%$ greater than the average rms obtained for the static case over the same angle of attack range, in which the rms is $0.013 \times / c$. In the static case, a Discrete Fourier Transform was performed at the maximum and minimum angles of attack of $29^{\circ}$ and $39^{\circ}$. No dominate frequency or harmonics are apparent in either case.

It was assumed that straight lines could be used to represent the vortex core in the angle of attack range of $29^{\circ}$ to $39^{\circ}$, and a vortex core inclination angle $(\beta)$ relative to the model surface was defined. At reduced frequencies of $k=0.05$ and 0.10 no hysteresis is observable in inclination angle $\beta$ when plotted against angle of attack over the range of pitching motion. However, a hysteresis does develop at a reduced frequency of $k=0.20$ over part of the cycle, and at $k=0.30$ a hysteresis exist over the entire cycle. At a given angle of attack, the inclination angle is less on the upstroke than it is on the downstroke. In the cases for which a hysteresis is evident, the effect is greater at higher angles of attack. 
When the wing was pitched through a large amplitude motion of $0^{\circ}$ to $45^{\circ}$ for reduced frequencies of $k=0.05$ and 0.30 , a hysteresis develops in the location of vortex breakdown and inclination angle, similar to that in the small amplitude cases. A hysteresis is evident at a reduced frequency as low as $k=$ 0.05 , and at a reduced frequency of $k=0.30$ the effect is much greater. A majority of the increase in hysteresis occurs on the downstroke phase of the cycle. Over this angle of attack range, flow separation and reattachment occur. As the angle of attack increases, some of the flow seems to separate all along the leading edge, while a majority of it stays attached. As the angle of attack continues to increase, the flow then transitions to completely separated flow. Flow reattachment appears to start at the apex and then propagates along the leading edge toward the trailing edge as the angle of attack decreases. No condition occurs for decreasing angle of attack in which the flow is both separated and attached as it did for increasing angle of attack.

A phase lag was also computed between the position of vortex breakdown and model angular position. The resulting phase lags are approximately equal to those obtained in the small amplitude case given the same reduced frequencies. Phase lags of $5^{\circ}$ and $22^{\circ}$ are obtained at reduced frequencies of $k=0.05$ and $k=0.30$ respectively.

The wing was also impulsively pitched-up from $28^{\circ}$ to $37^{\circ}$ degrees and impulsively pitched-down from $37^{\circ}$ to $27^{\circ}$ at an approximate nondimensional pitch rate of $K=0.016$ and freestream Reynolds number of 260,000 . The response of the vortex flow to the model motion is immediate, but upon completion of the motion, from 4 to 7 convective times are required to reach a steady state. During the motion, the convective time lag is approximately 1 time unit. 
Lastly, the spanwise location of vortex breakdown varies by approximately $3 \%$ as the wing sinusoidally oscillates between $29^{\circ}$ and $39^{\circ}$. The values range from approximately $0.58 \mathrm{y} / \mathrm{s}$ to $0.61 \mathrm{y} / \mathrm{s}$. On the average, the spanwise location of vortex breakdown moves outward as angle of attack increases.

\subsection{Recommendations}

In this study, several analyses on the dynamics of the vortex flow due to various model motions were performed, and many interesting results were obtained. However, several questions were left unanswered and additional testing is needed to help answer these questions. Several recommendations for future research are presented below. They include both modifications to the experimental apparatus, which could improve the data acquisition procedure, as well as suggestions for additional experimental investigations.

1. Changes which could improve the experimental test facility are as follows:

-- The addition of an angular displacement transducer. This would increase the accuracy of the angle of attack measurements and also reduce the time required for data reduction. The accuracy of the harmonic distortion calculation would also be increased, and two cameras would no longer be needed for the collection of spanwise breakdown data.

-- The use of a video taping system for data collection. By using video tape, the number of pitching cycles which could be recorded would be virtually "unlimited," and the quality of the flow visualization could be viewed "real time." The video tape images would then be projected 
onto the digitizing table frame by frame using a projection video monitor and editing tape deck.

2. Why did the dynamic behavior of vortex breakdown significantly change between the Reynolds numbers of 175,000 and 260,000? To further investigate and verify this phenomenon, additional tests should be performed in the range of Reynolds number over which the change occurred. In addition, a range of Reynolds numbers higher than those examined in the present study should be investigated to determine if similar changes occur at higher Reynolds numbers.

3. Another aspect of the experiment which should be determined is the effect of a change in pitching axis on the dynamic behavior of vortex breakdown. In the present investigation the response of the position of vortex breakdown was quantified for the wing being pitched about the 1/2 chord position for a range of reduced frequencies and Reynolds numbers. It would be of interest to quantify the effects and/or differences (if any) of pitching the wing at the $1 / 4$ chord and/or $3 / 4$ chord positions for the same range of reduced frequencies and Reynolds numbers.

4. Additional testing should be performed on the response of the spanwise location of vortex breakdown due to dynamic motion. More specific information should be obtainable using the synchronous $35 \mathrm{~mm}$ photography setup with slight modifications to the experimental facility, or by implementing the modifications which are discussed in paragraph 1 above. 
5. A more in depth study should be performed on the response of the vortex flow due to an impulsive change in angle of attack. The effect of different angle of attack time histories and angle of attack ranges should also be determined, with emphasis placed on the evaluation of the lag times involved with the vortex flow in relation to model motion.

6. A lower range of frequencies should be examined in the Discrete Fourier Transform of the oscillation of the chordwise breakdown location. The lowest frequency examined in the present study was $1 \mathrm{~Hz}$ and no dominate frequency was observed. The chance that a lower dominate frequency exists is possible.

7. And finally, it would be of considerable importance to couple the visual data obtained in this study with pressure surface data, or force balance data obtained at the same test conditions. Using the combination of the two types of data, a correlation between vortex breakdown and aerodynamic forces could be obtained for a wing undergoing dynamic motions. 


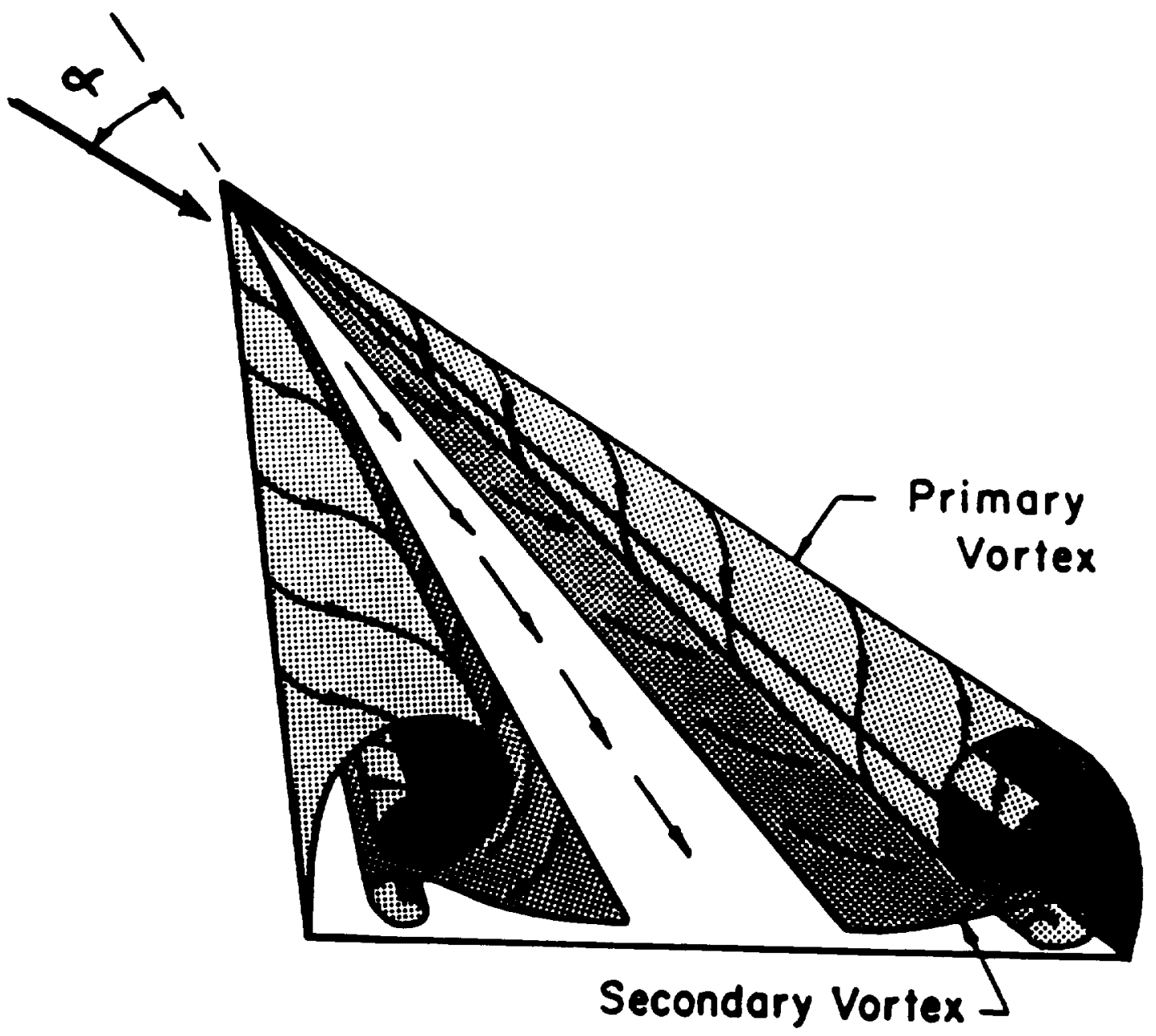

Figure 1.1: Vortex Flow over a Delta Wing. 


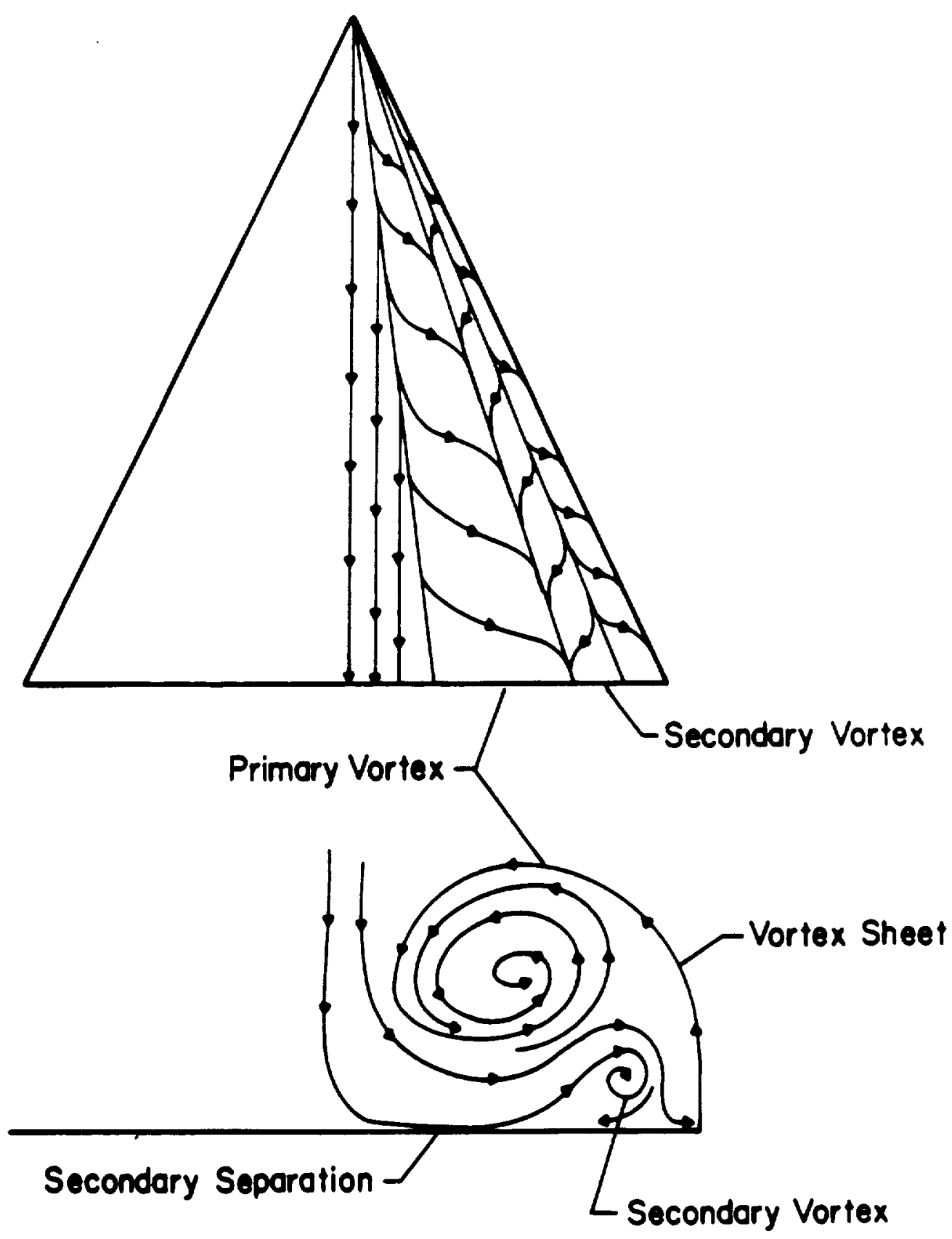

Figure 1.2: Schematic of Leading Edge Flow Patterns. 


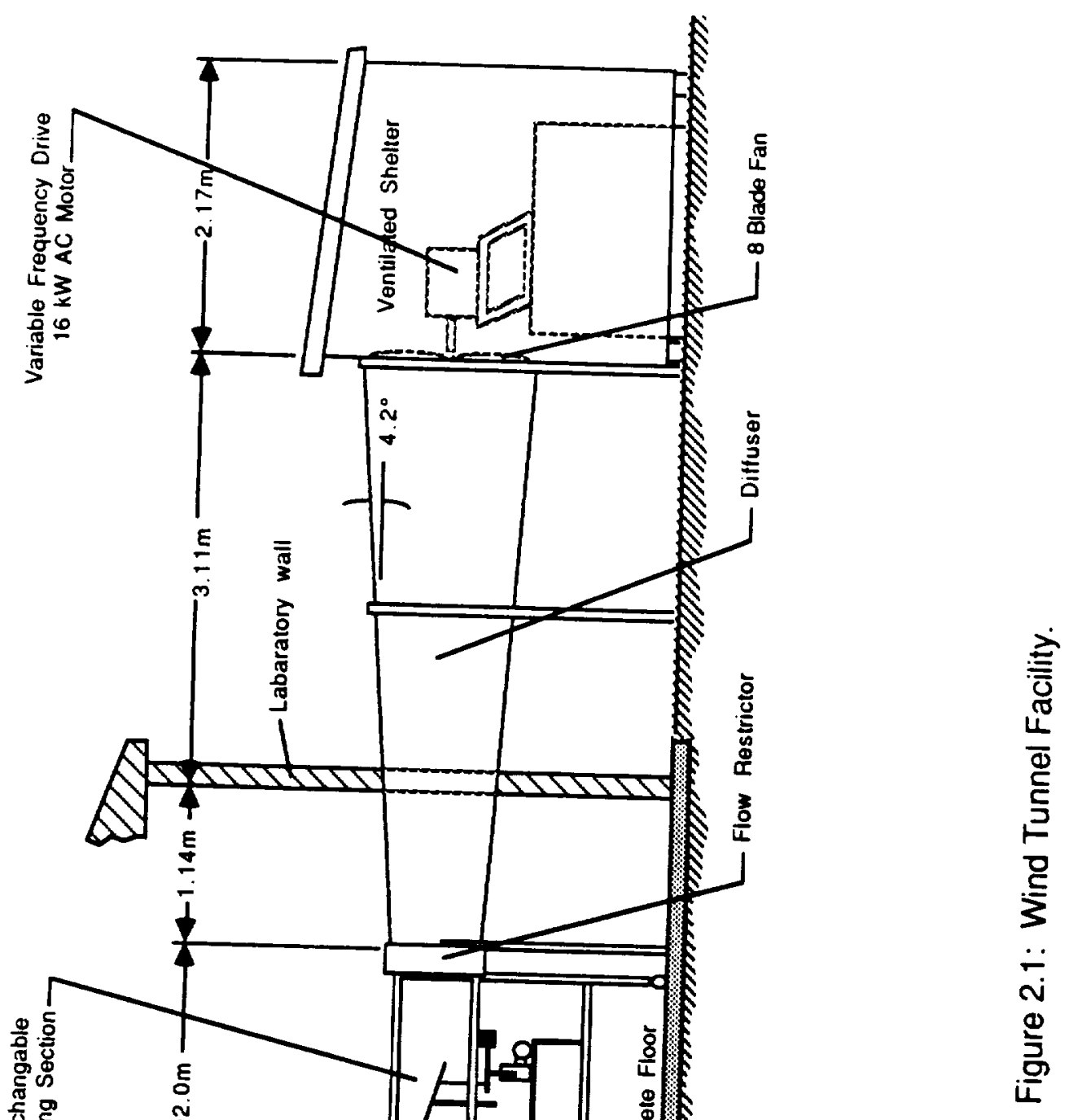


$-$

$-$

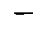

$-$

$-$
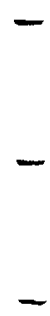

$-$
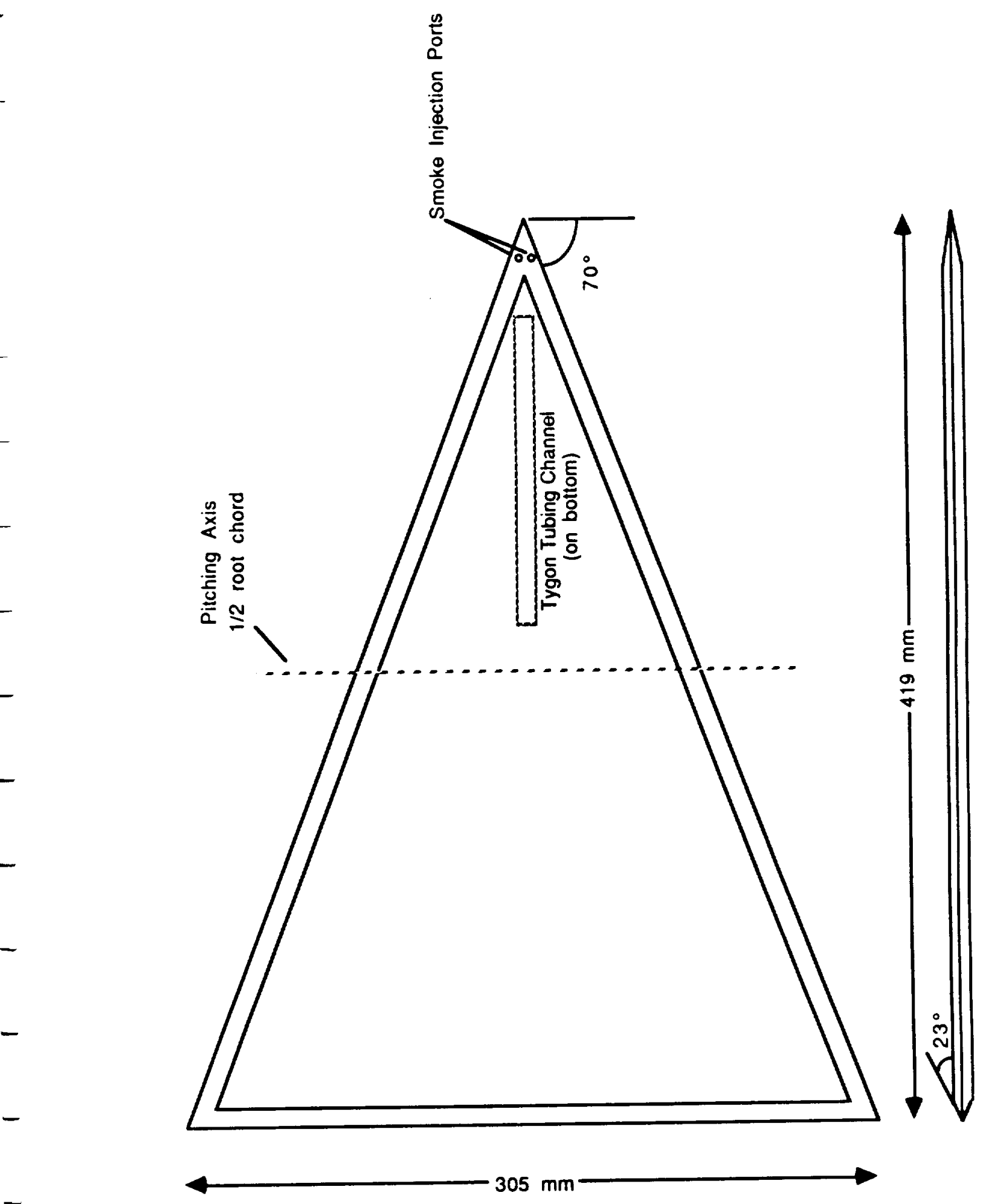

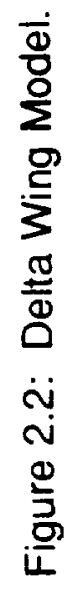



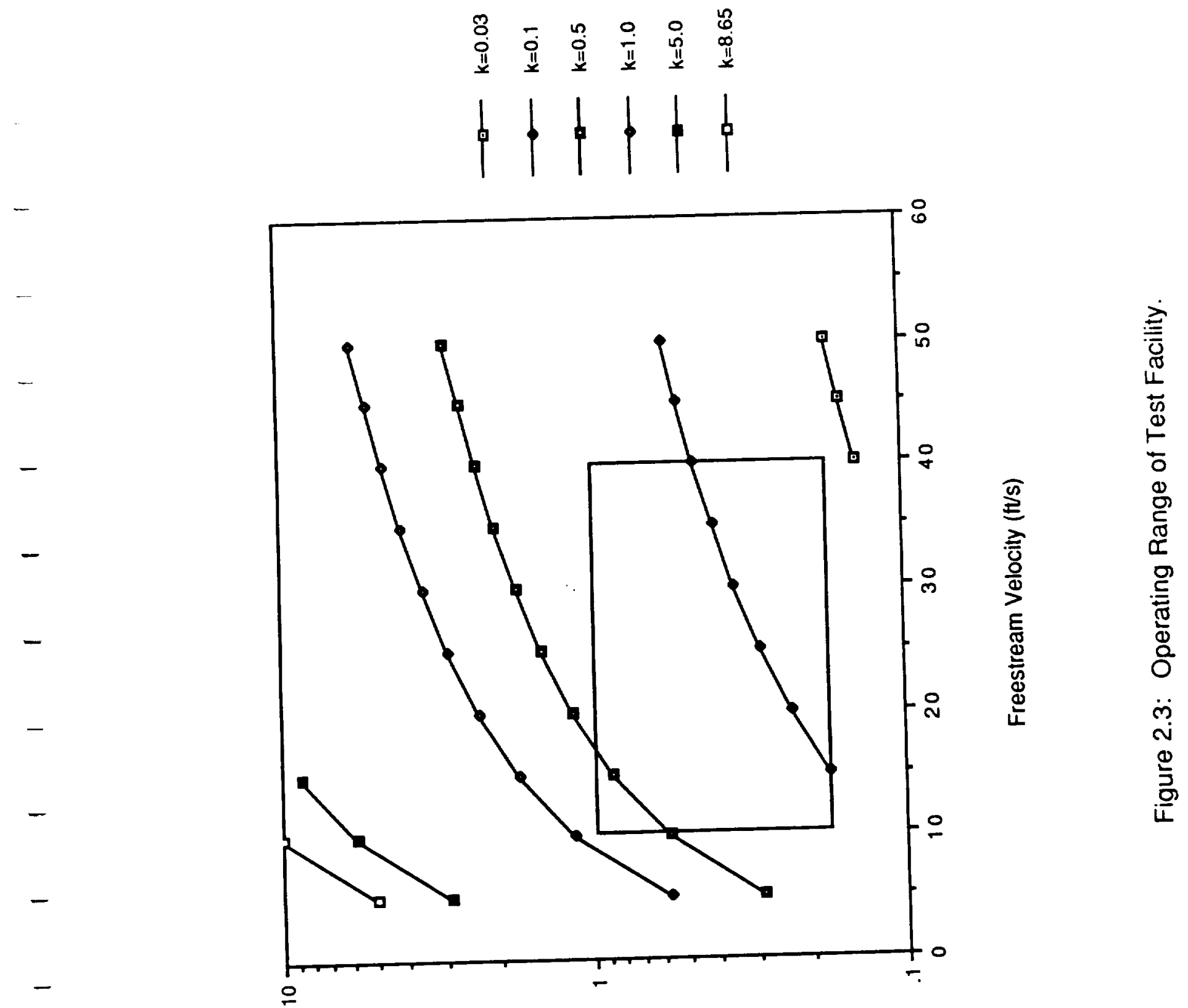

(ZH) Kouanbady Bu!4ग!!d 
Figure 2.4: Schematic of Delta Wing Pitching Mechanism.

Delta Wing Model

Model Mount 


\section{- ORIGINAL PAGE IS \\ OF POOR QUALITY \\ IS}

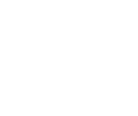

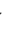

$-$
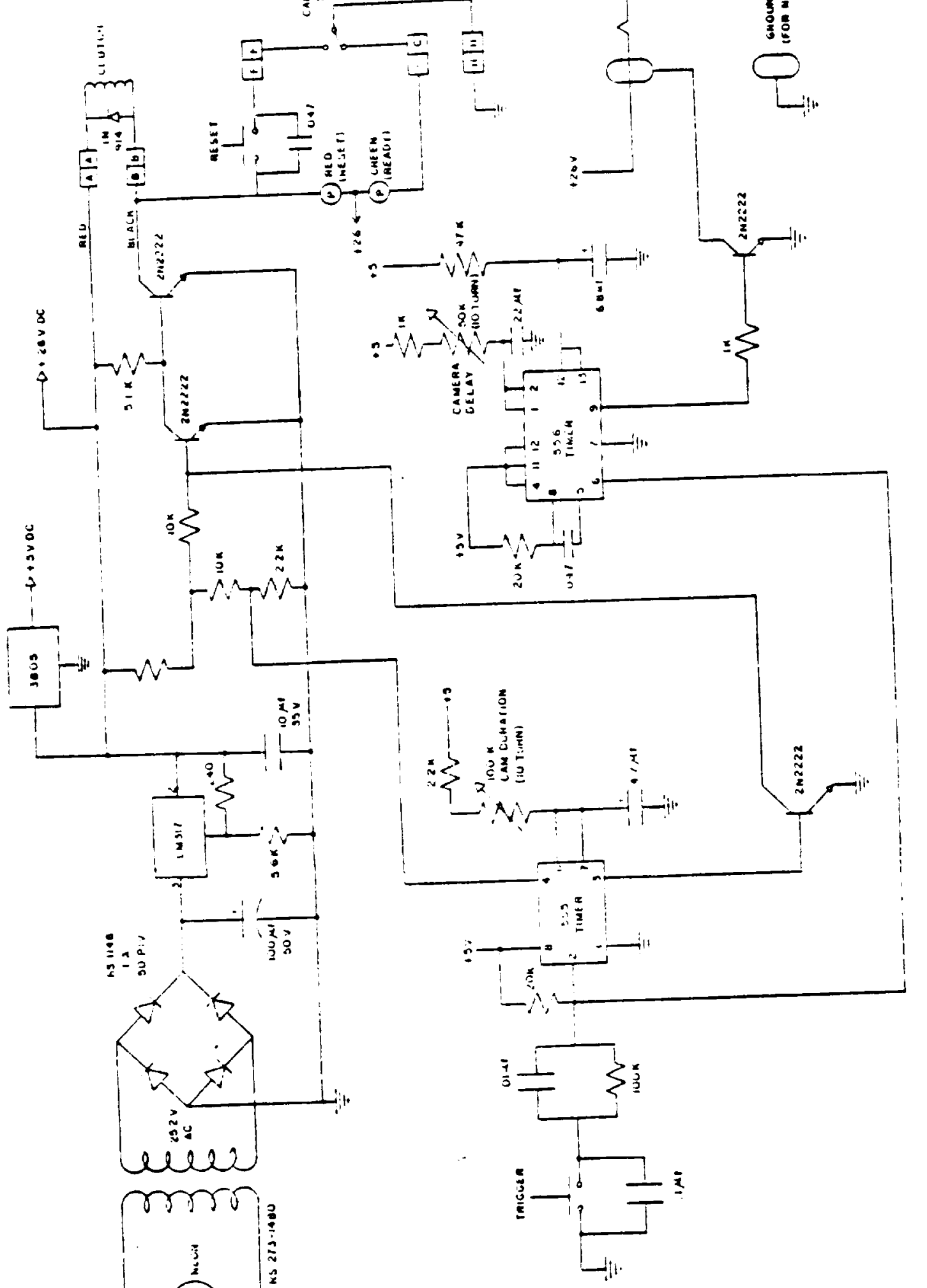

$\sqrt{2} \frac{1}{2}$

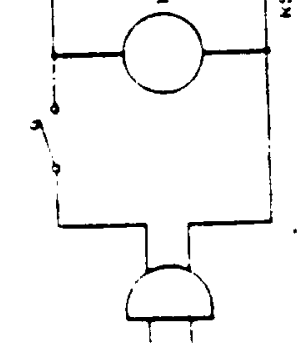

Figure 2.5: Schematic of Pitching Mechanism Control Box. 

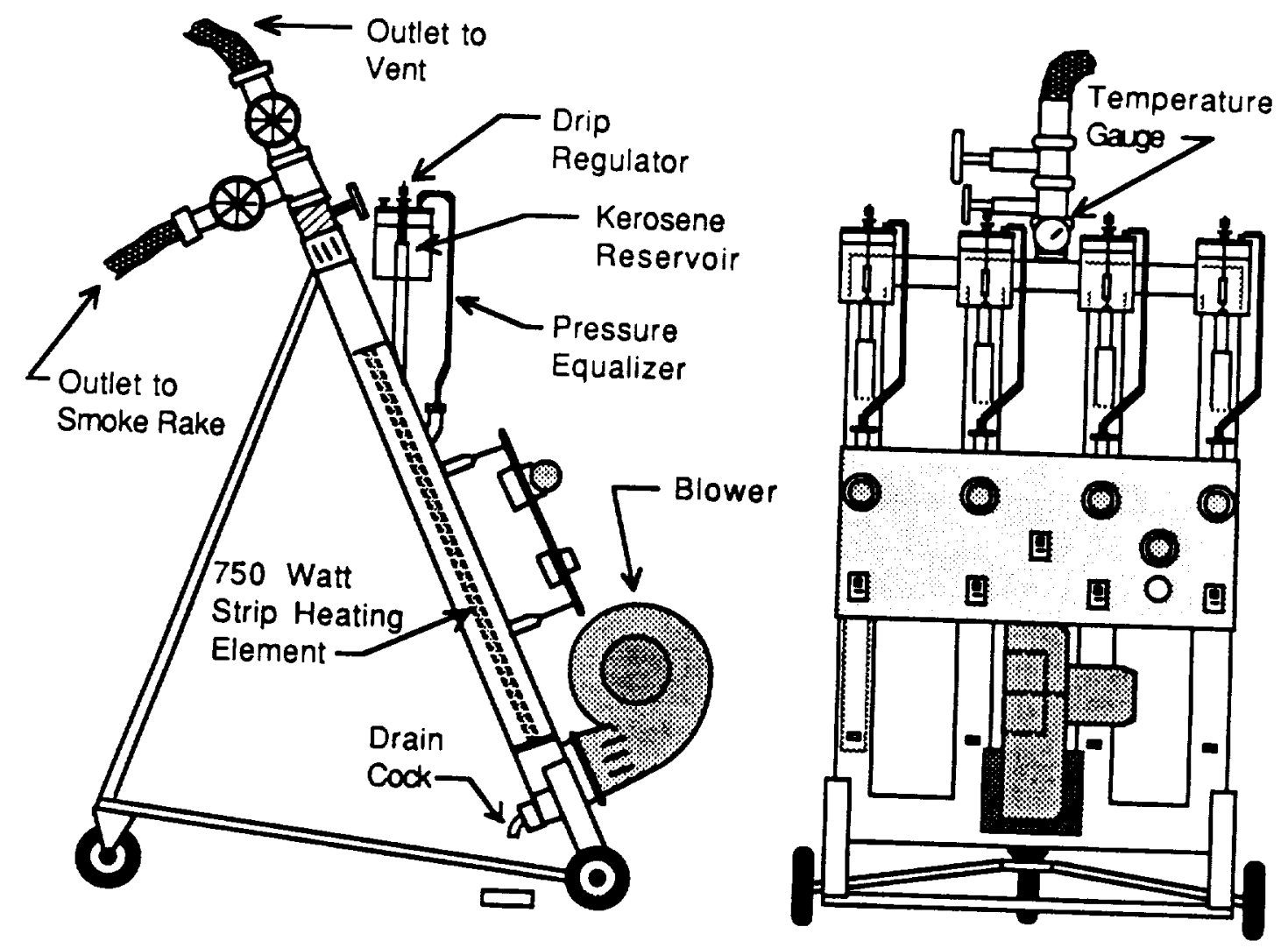

Figure 2.6: Kerosene Smoke Generator. 


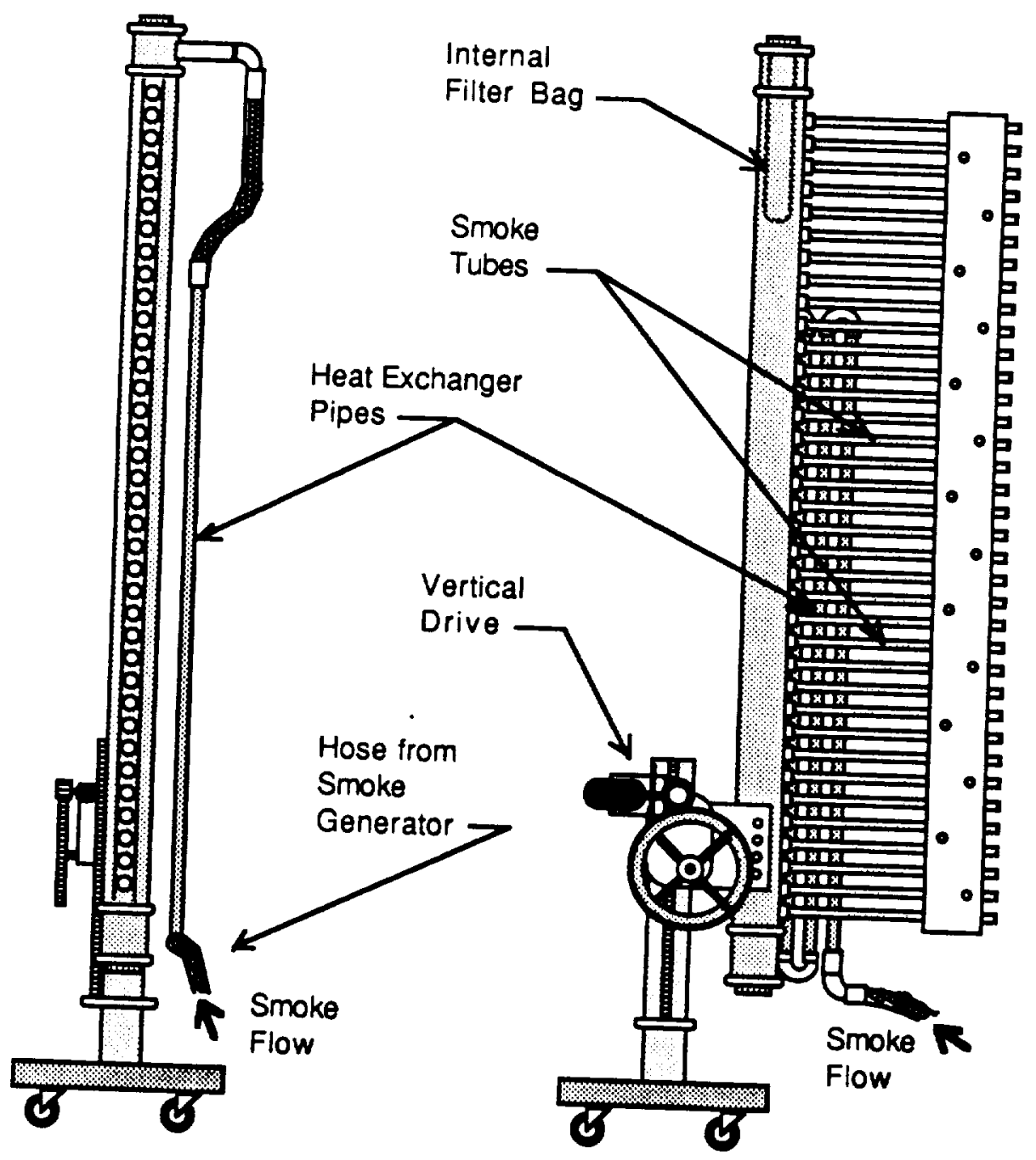

Figure 2.7: Smoke Rake. 

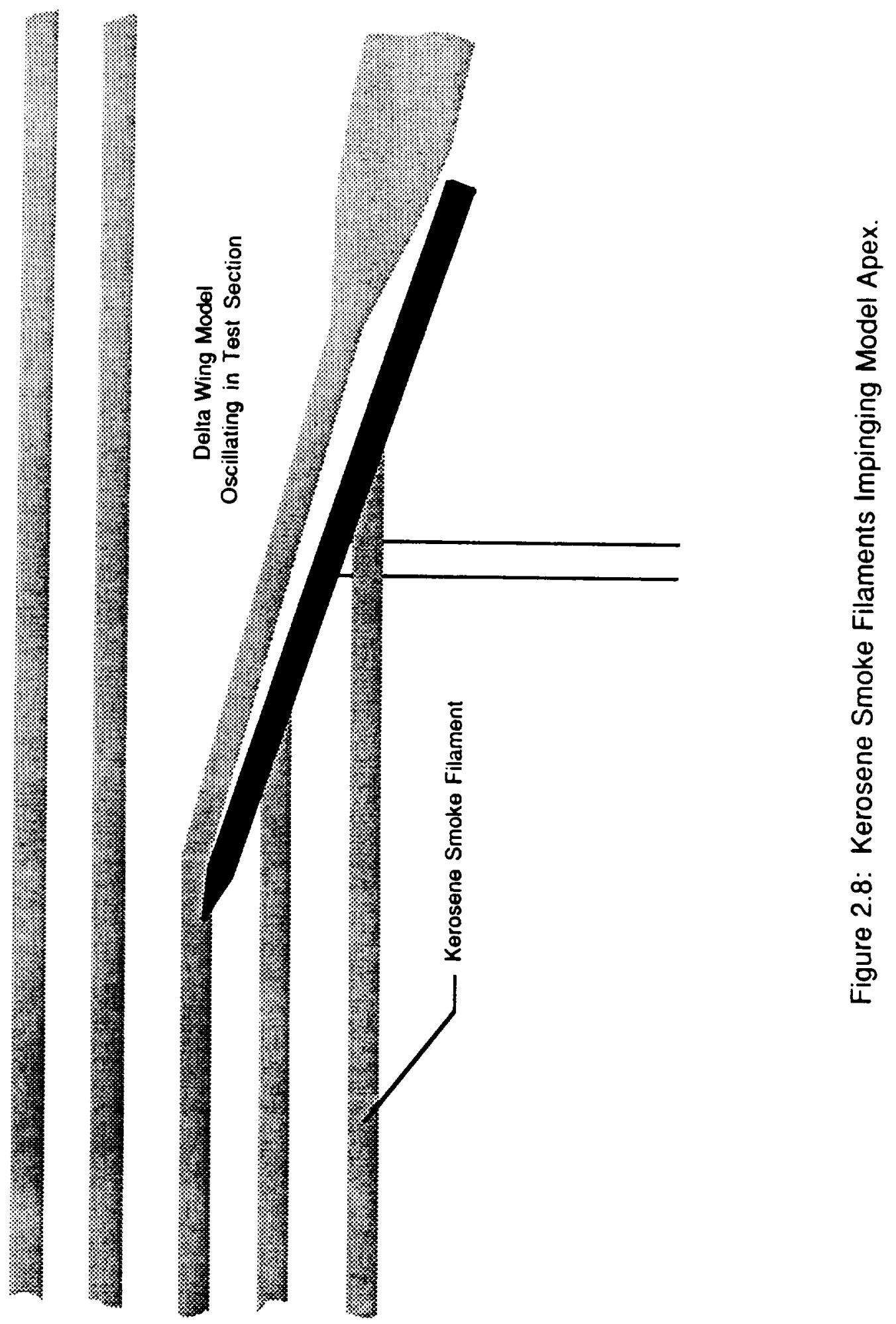


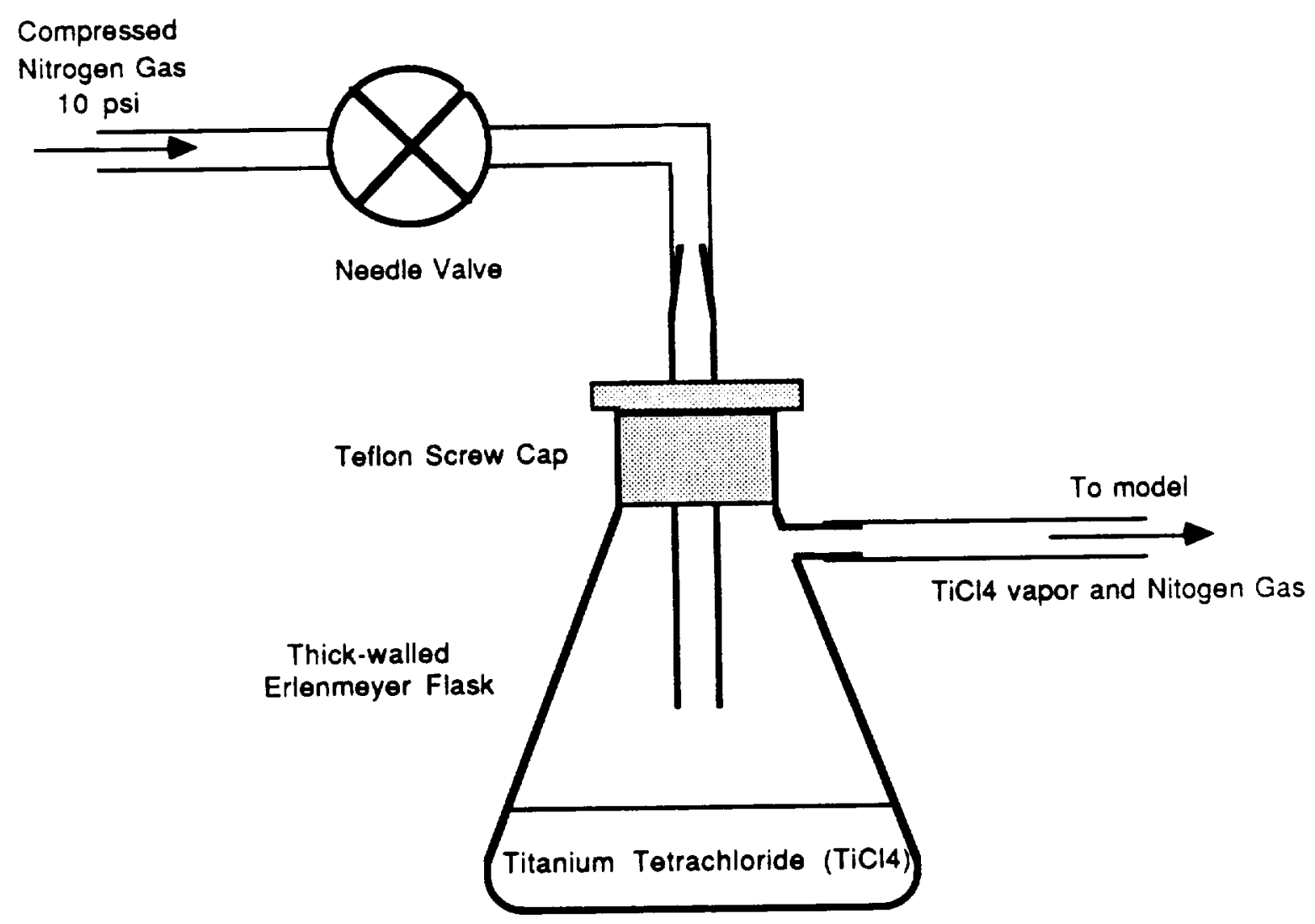

Figure 2.9: Titanium Tetrachloride Injection System. 


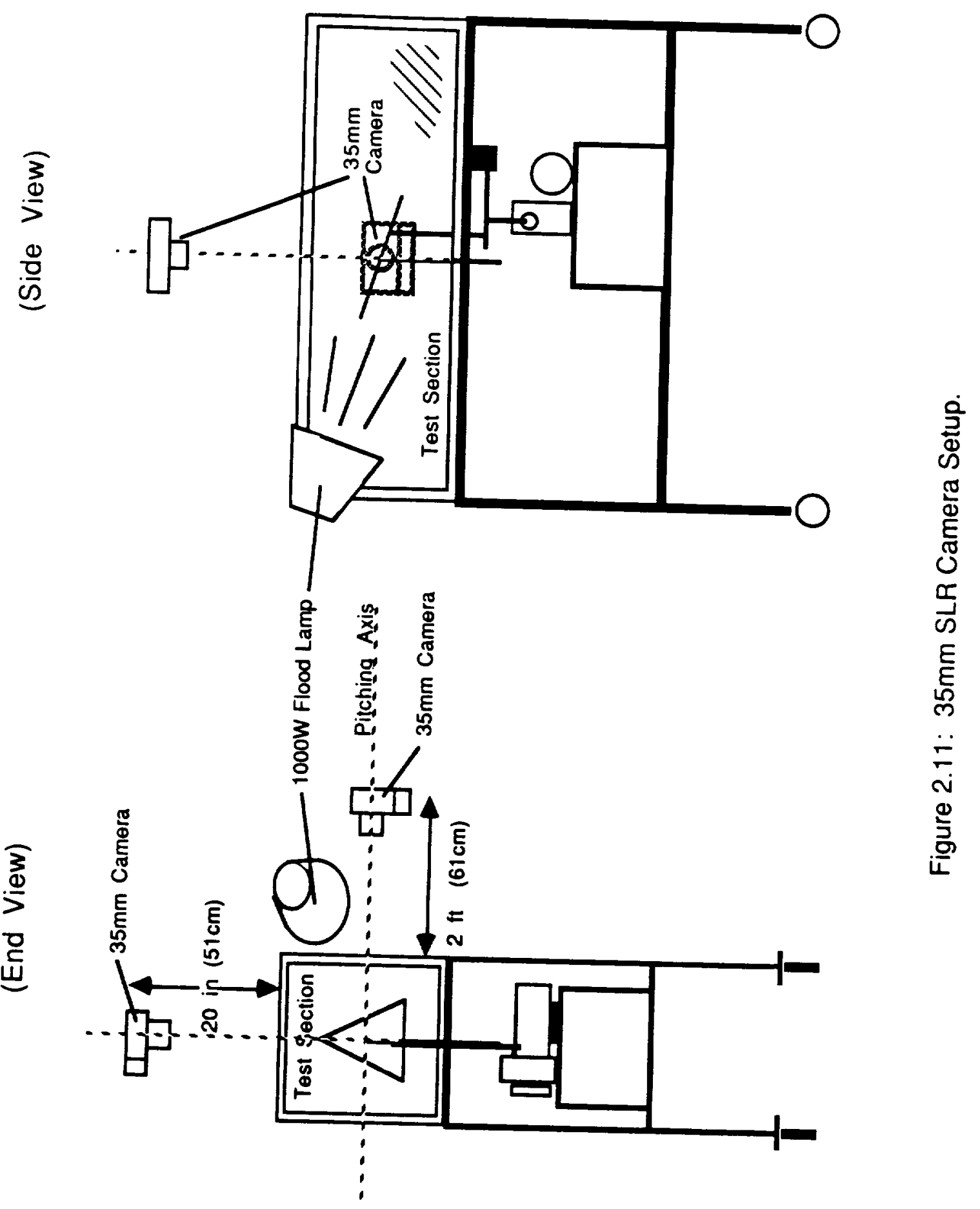



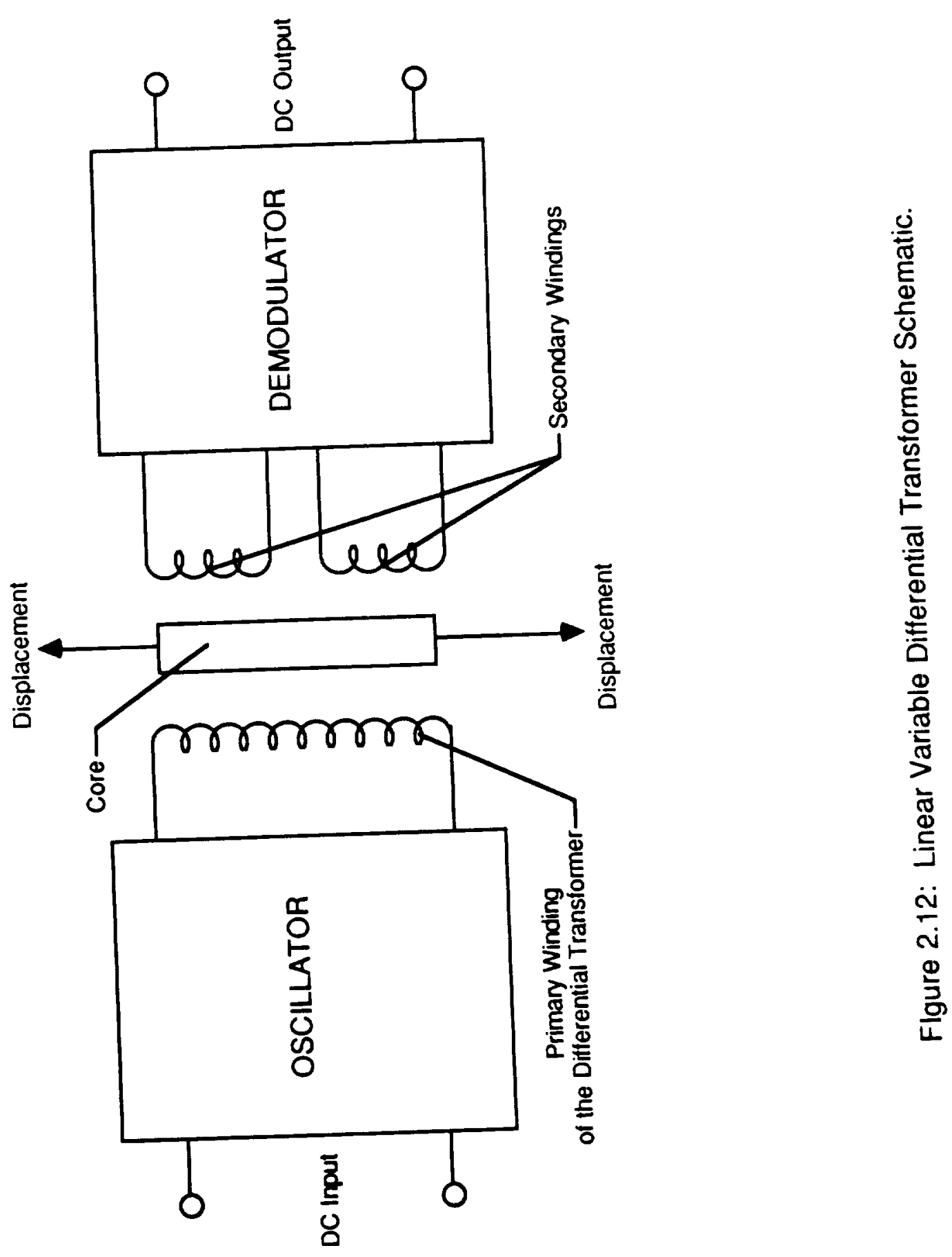
ORIGINAL PAGE

BLACK AND WHITE PHOTOGRAPH

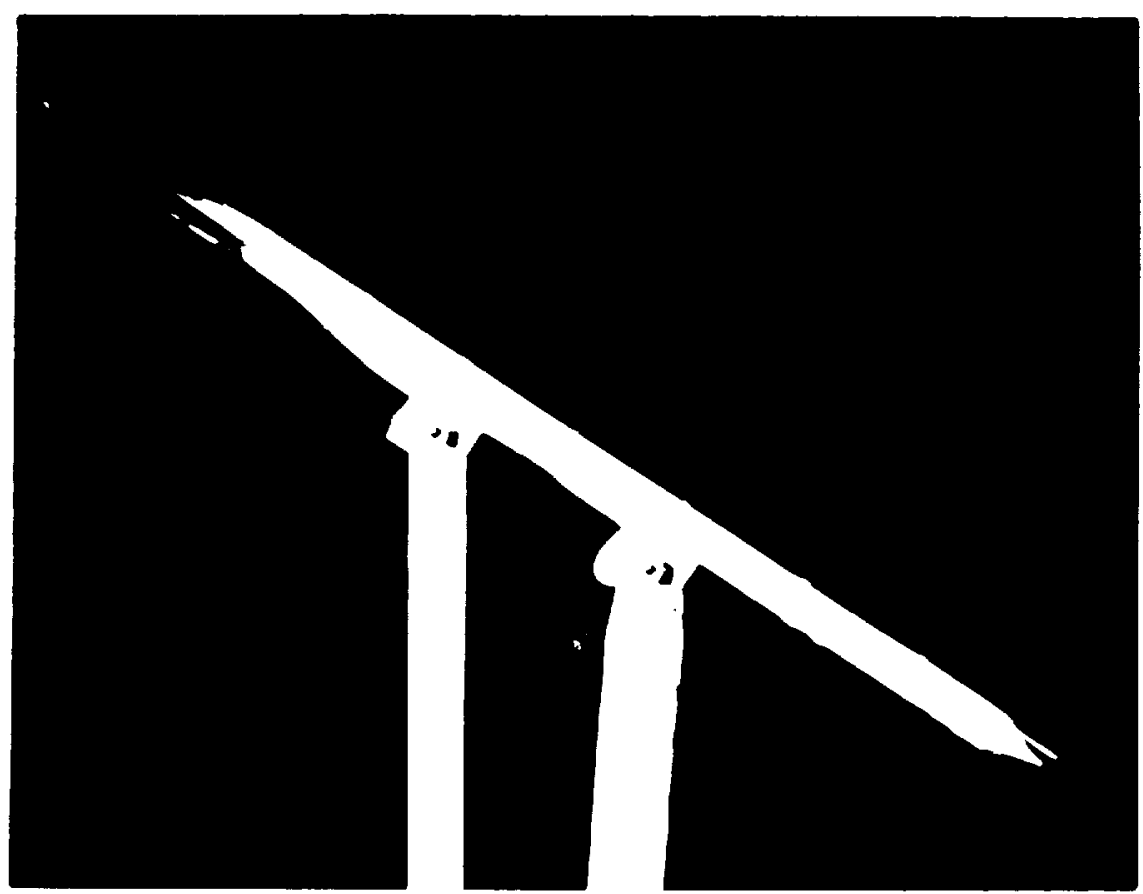

Figure 3.1: Example of a Photographic Image obtained from a Side View.

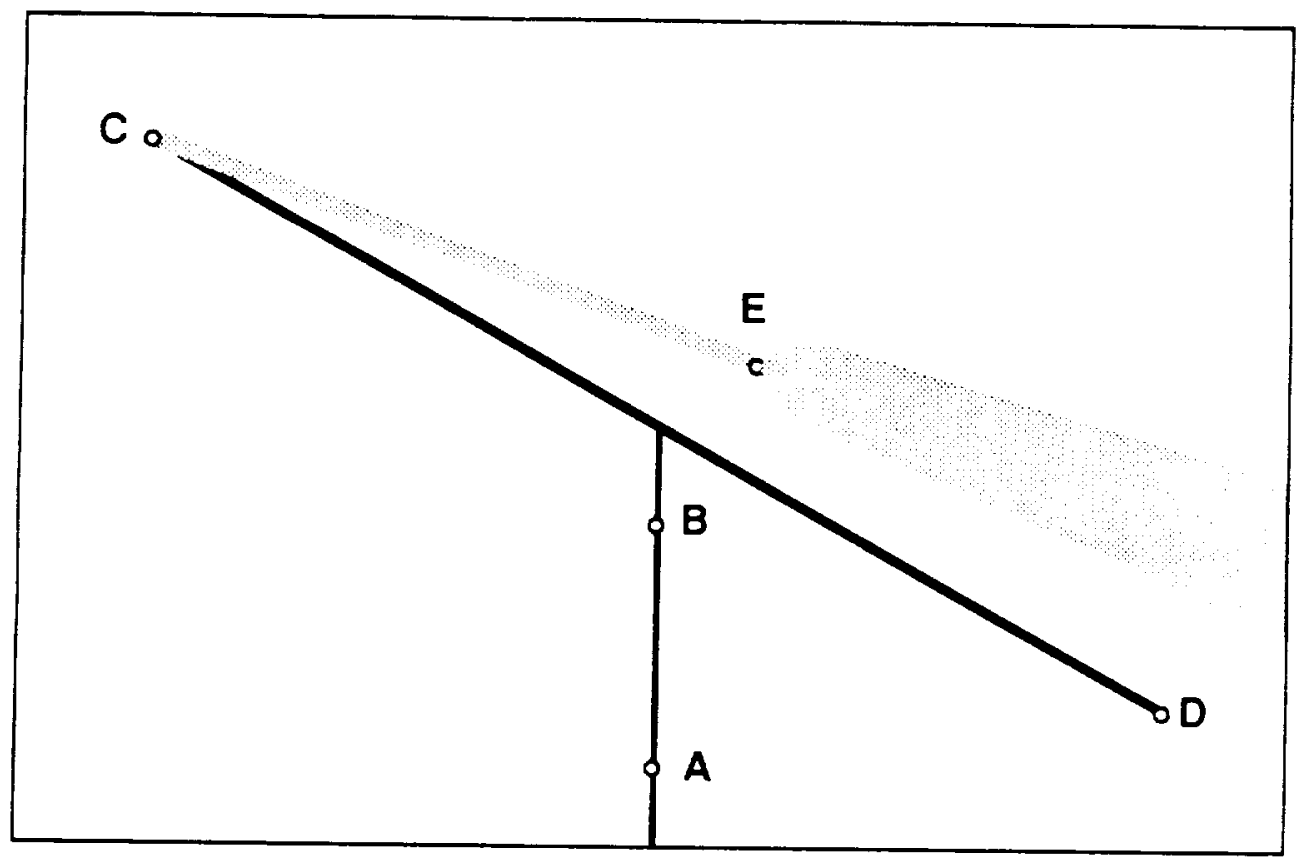

Figure 3.2: Schematic of a Photographic Image obtained from a Side View. 


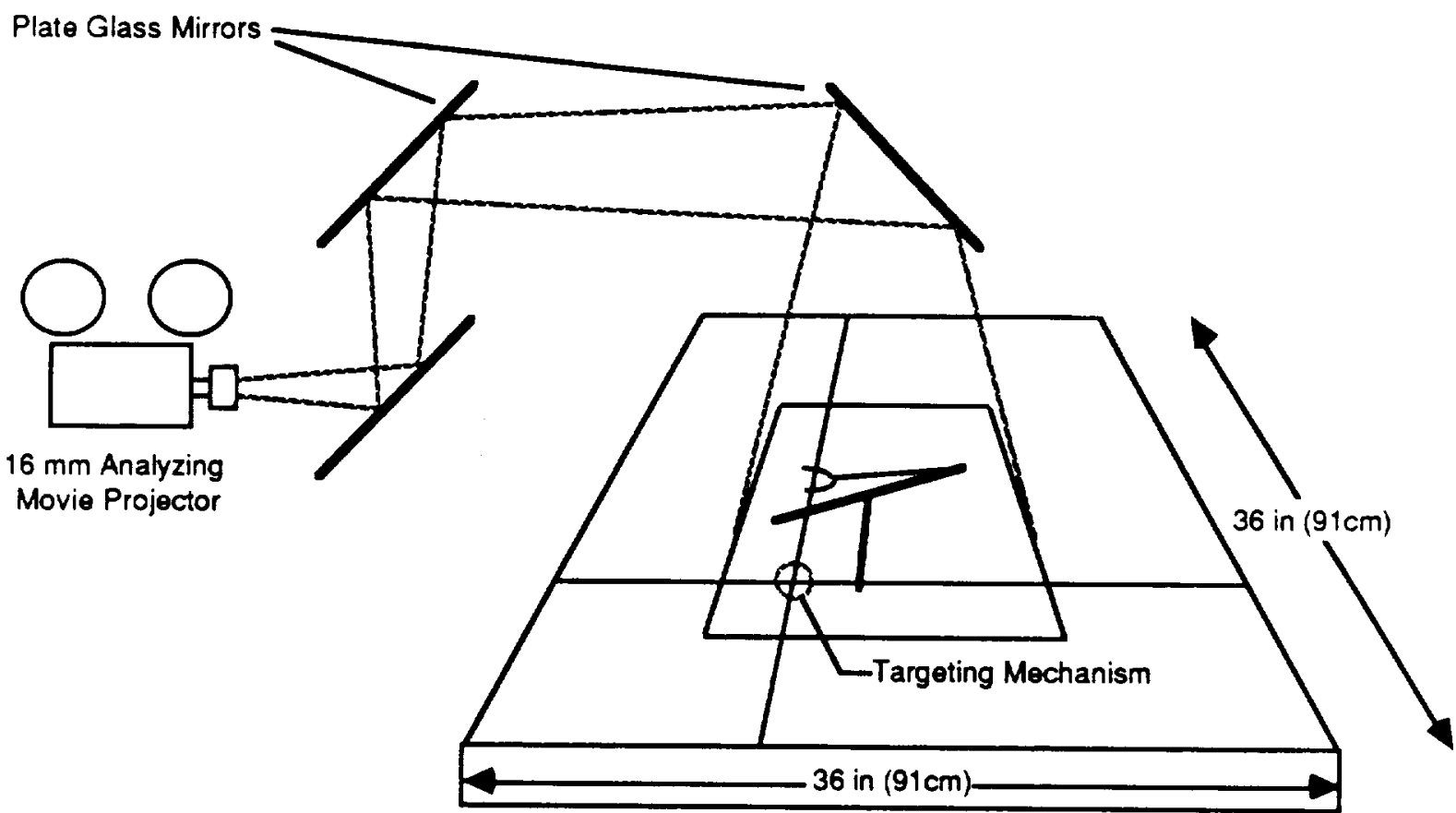

Wayne-George Digitizing Table 
BLACK AND WHITE PHOTOGRAPH

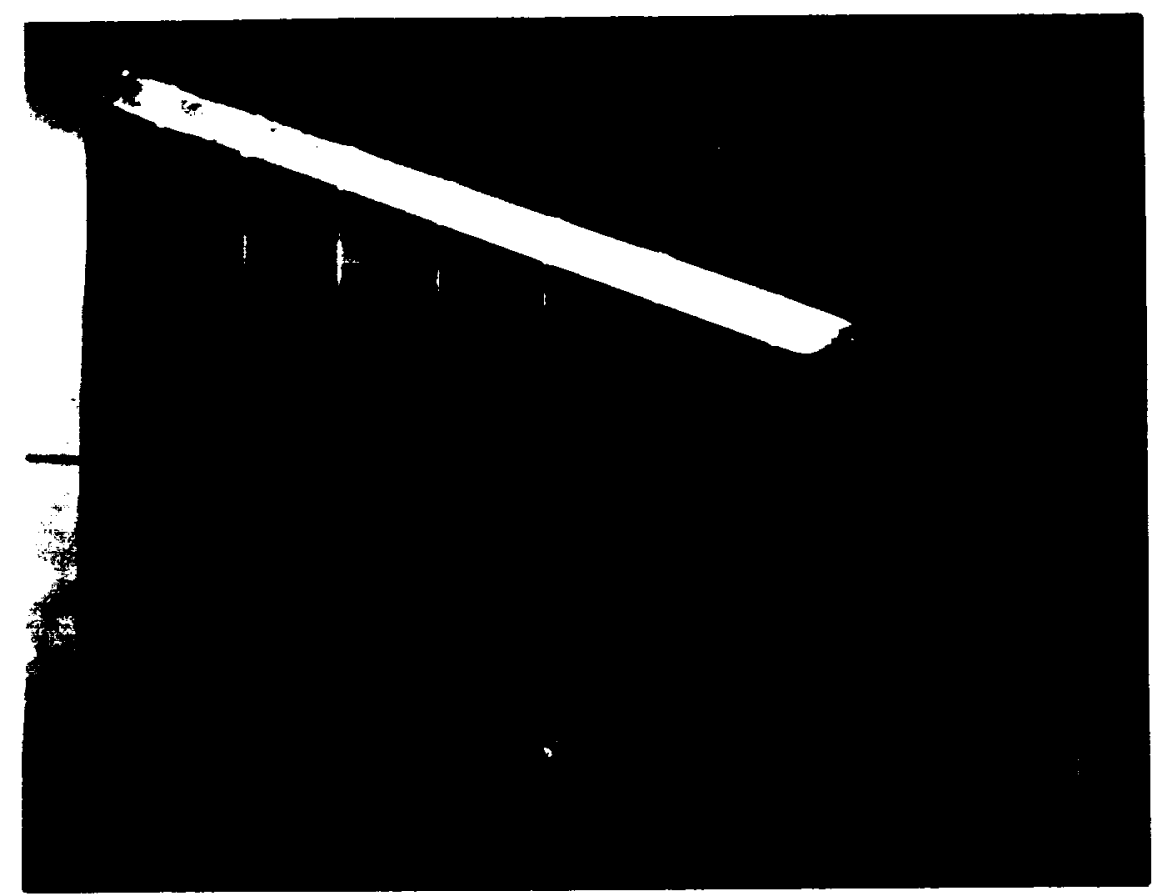

Figure 3.4: Example of Photographic Image obtained from a Top View.

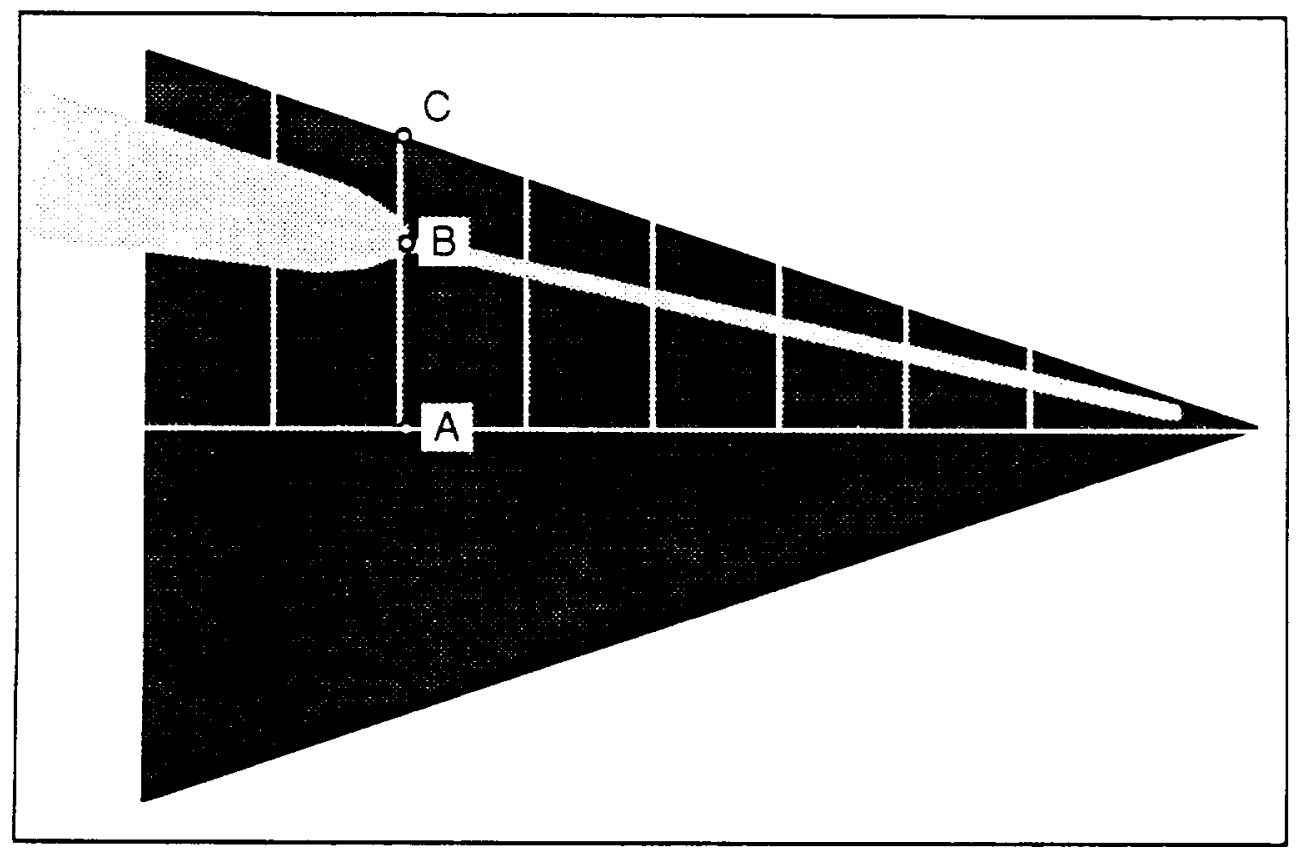

Figure 3.5: Schematic of Photographic Image obtained from a Top View. 


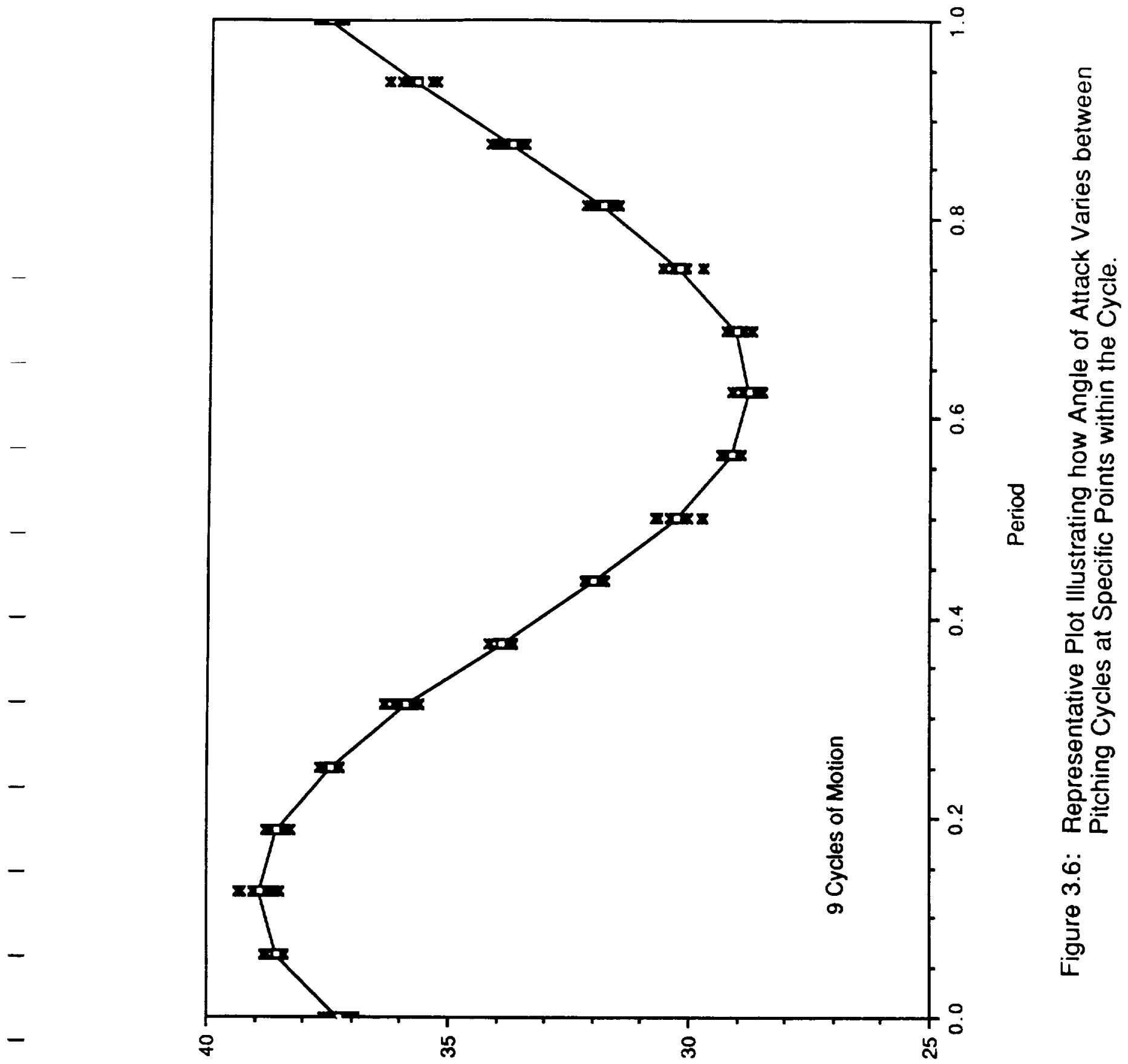

yoeแt to e|6uళ 


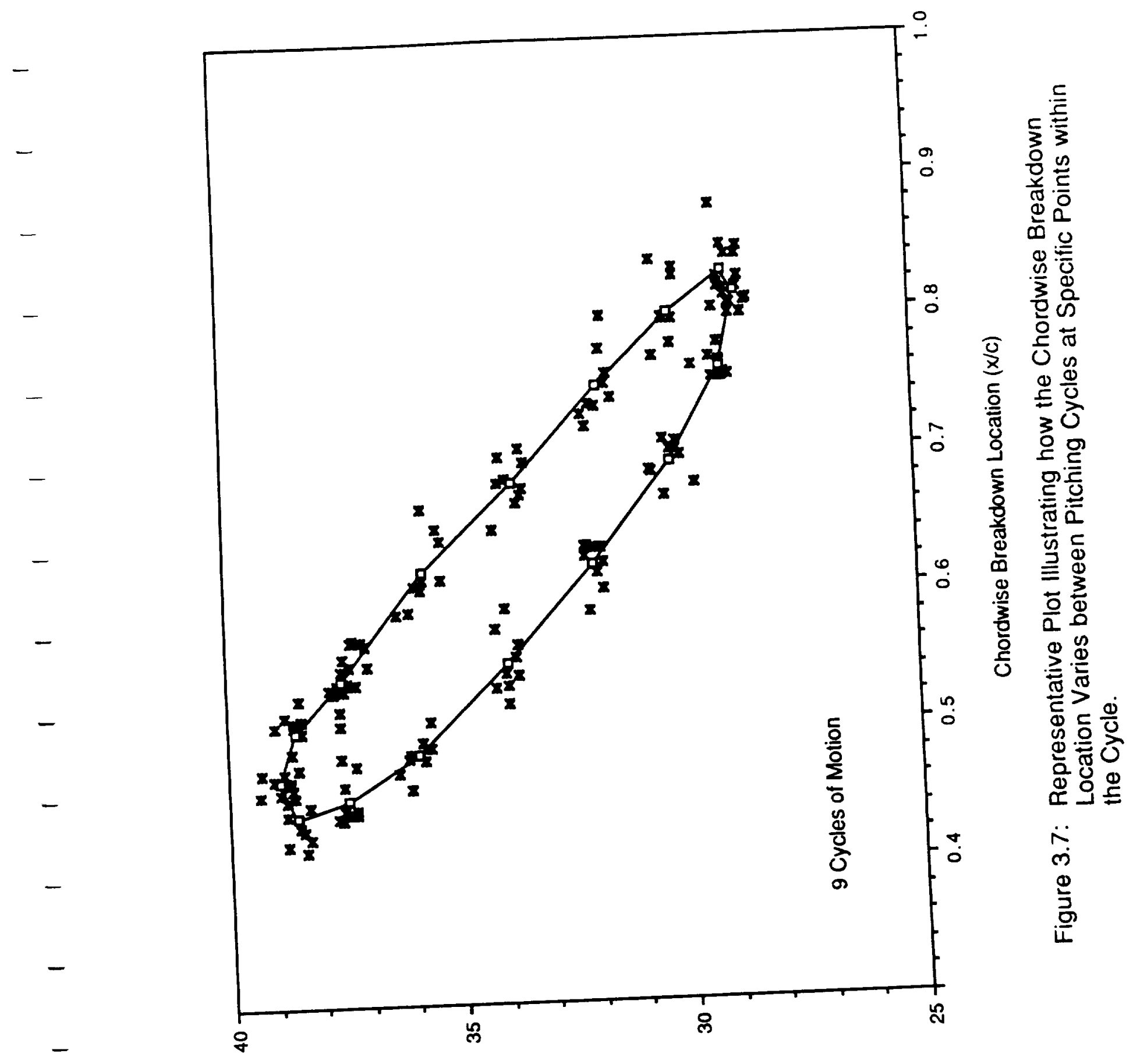

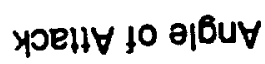




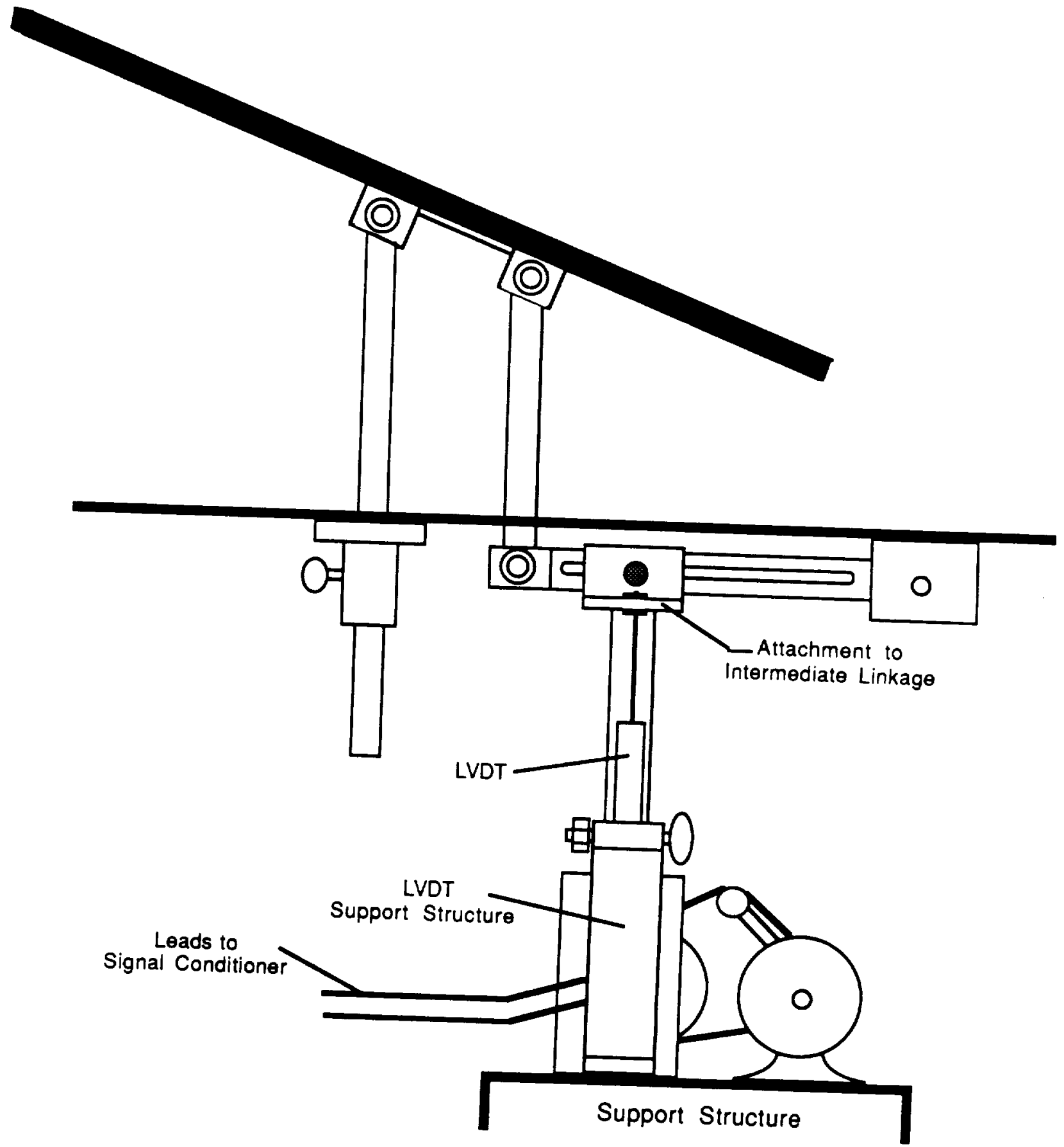

Figure 4.1: Attachment of LVDT to Delta Wing Pitching Mechanism. 


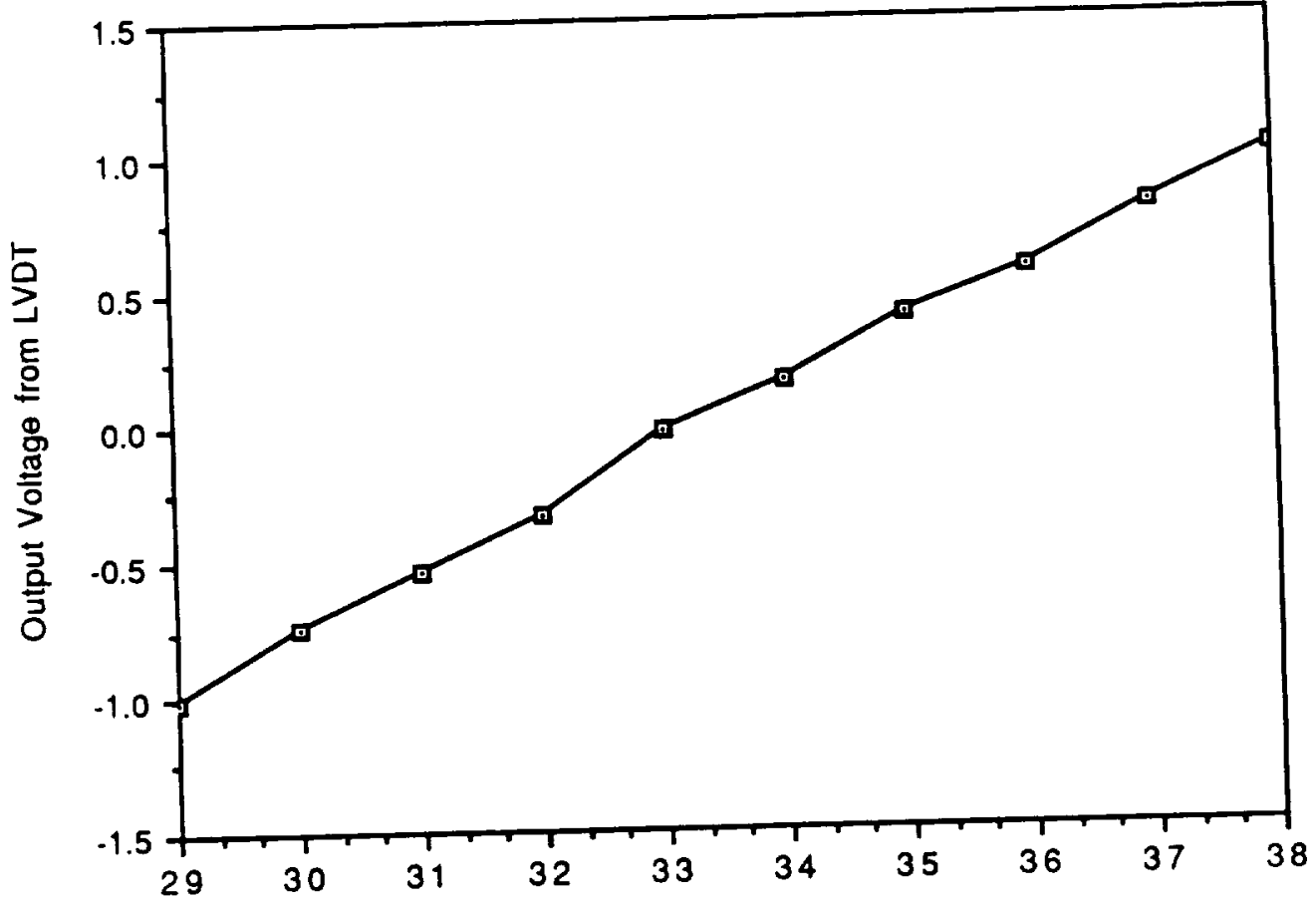

Angle of Attack

Figure 4.2: LVDT Calibration Curve, $29^{\circ}$ to $38^{\circ}$. 


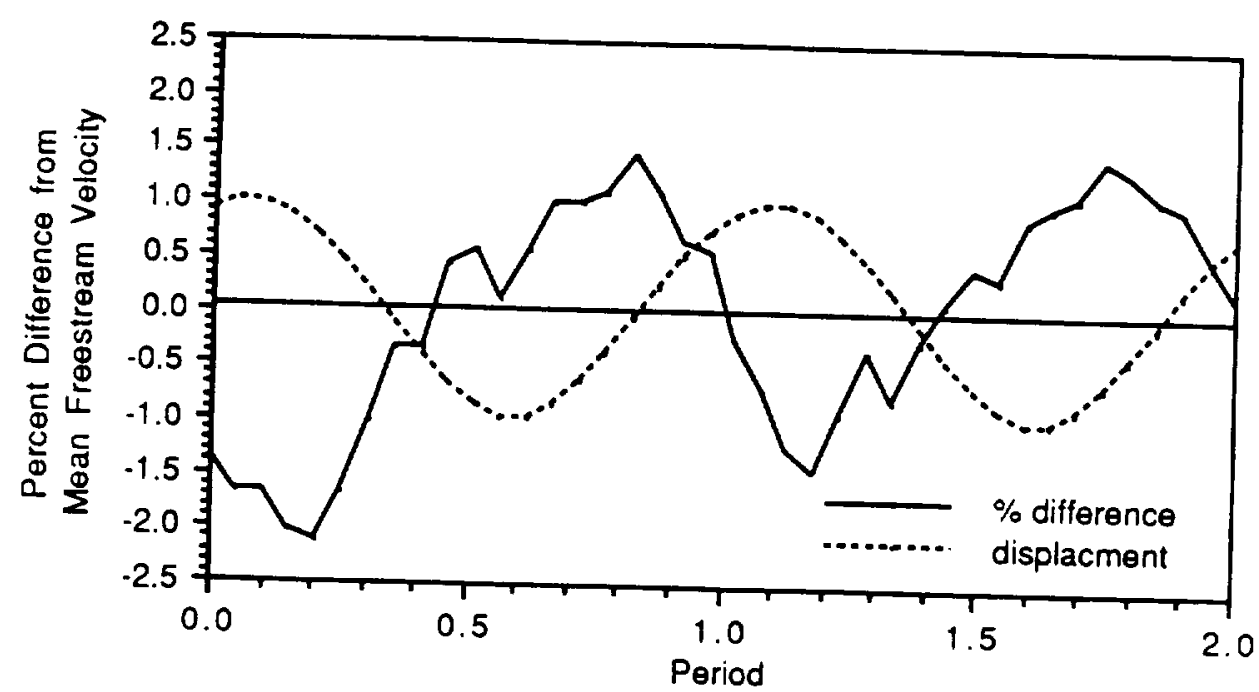

Figure 4.3a: One Data Record of the Freesteam Response due to Model Motion
for a Representative Case.

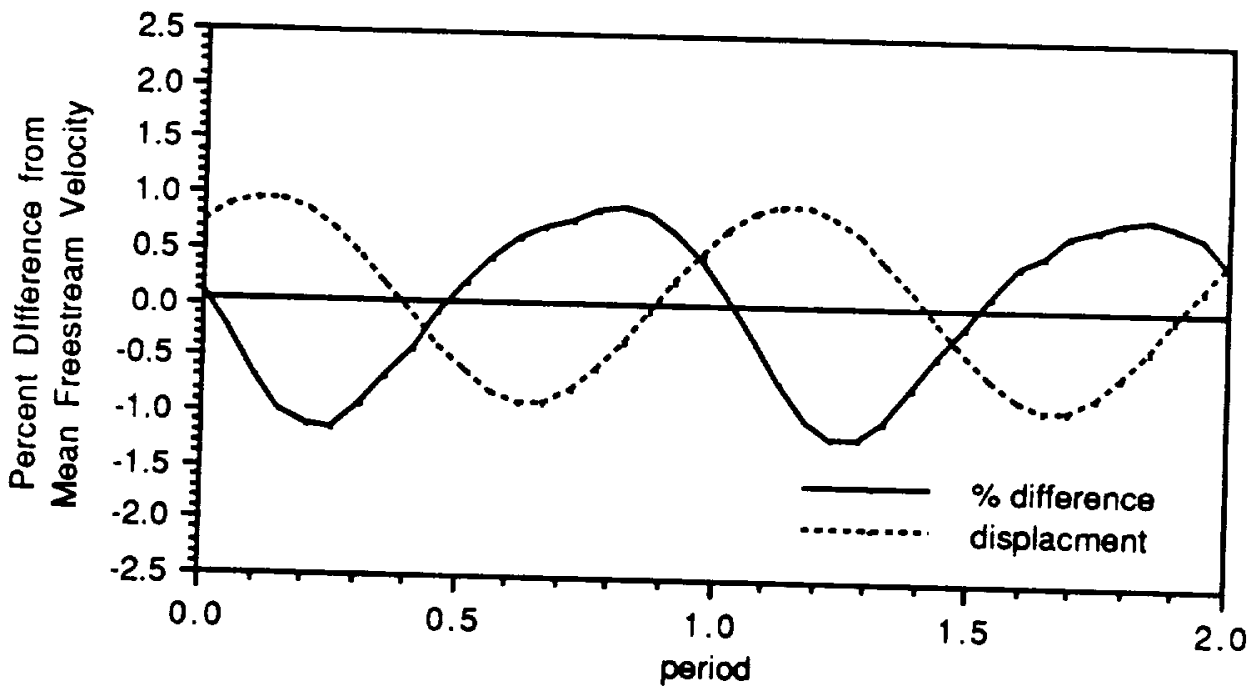

Figure 4.3b: Ensemble Average of 100 Data Records of the Freestream Response due to Model Motion for a Representative Case. 


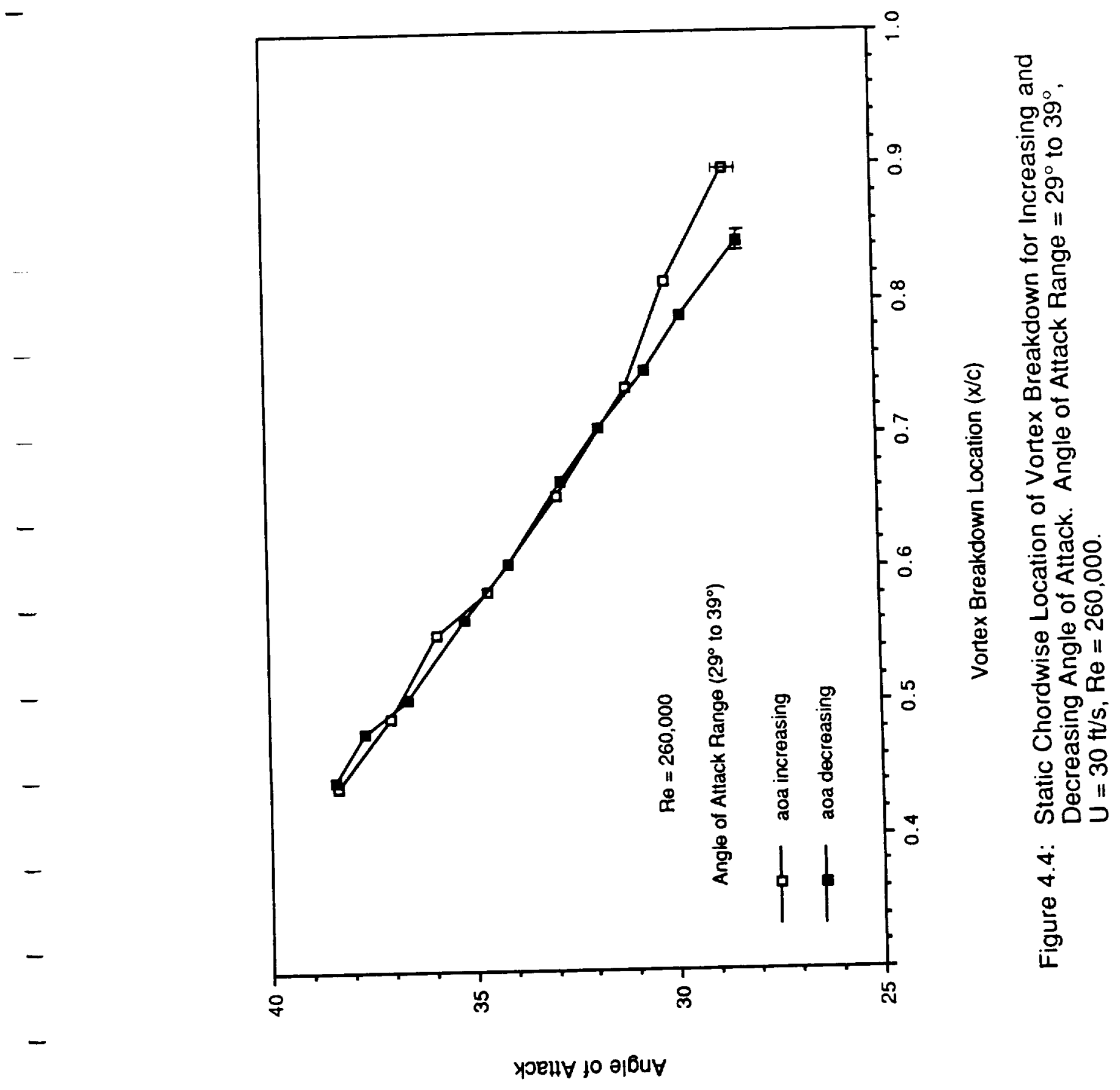




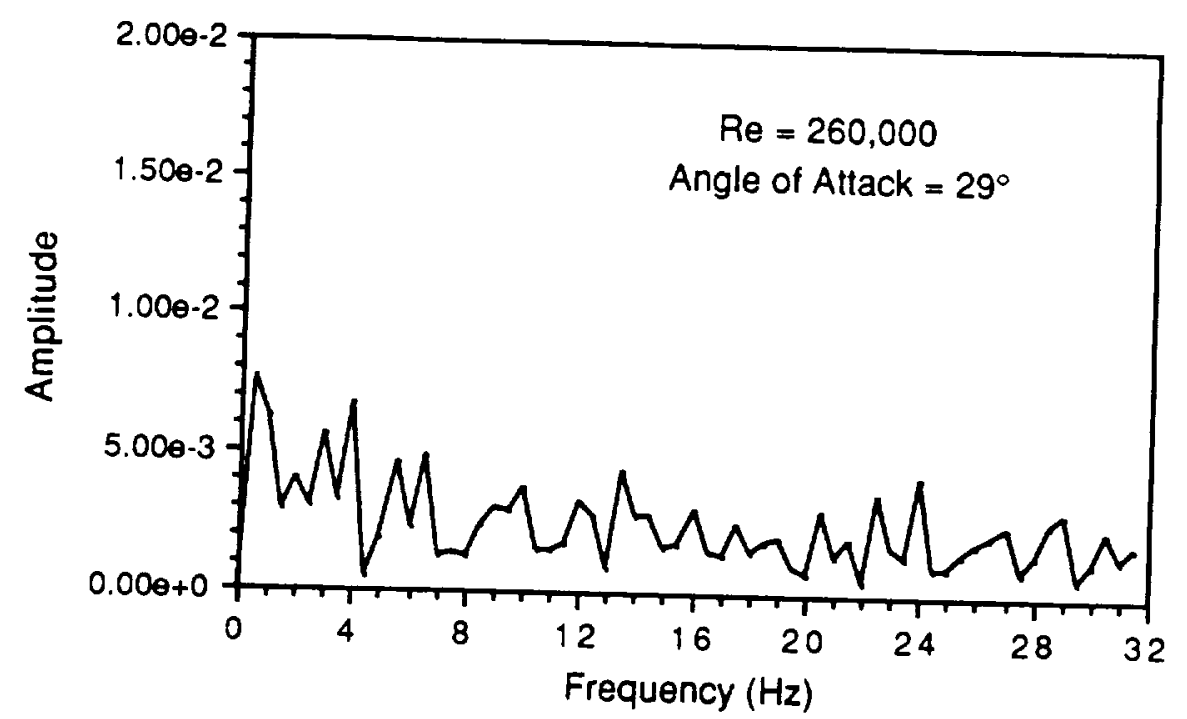

Figure 4.5a: Discrete Fourier Transform of the Variation in Chordwise Breakdown Location from Mean Position at an Angle of Attack of $29^{\circ}$. $U=30 \mathrm{ft} / \mathrm{s}, R e=260,000$.

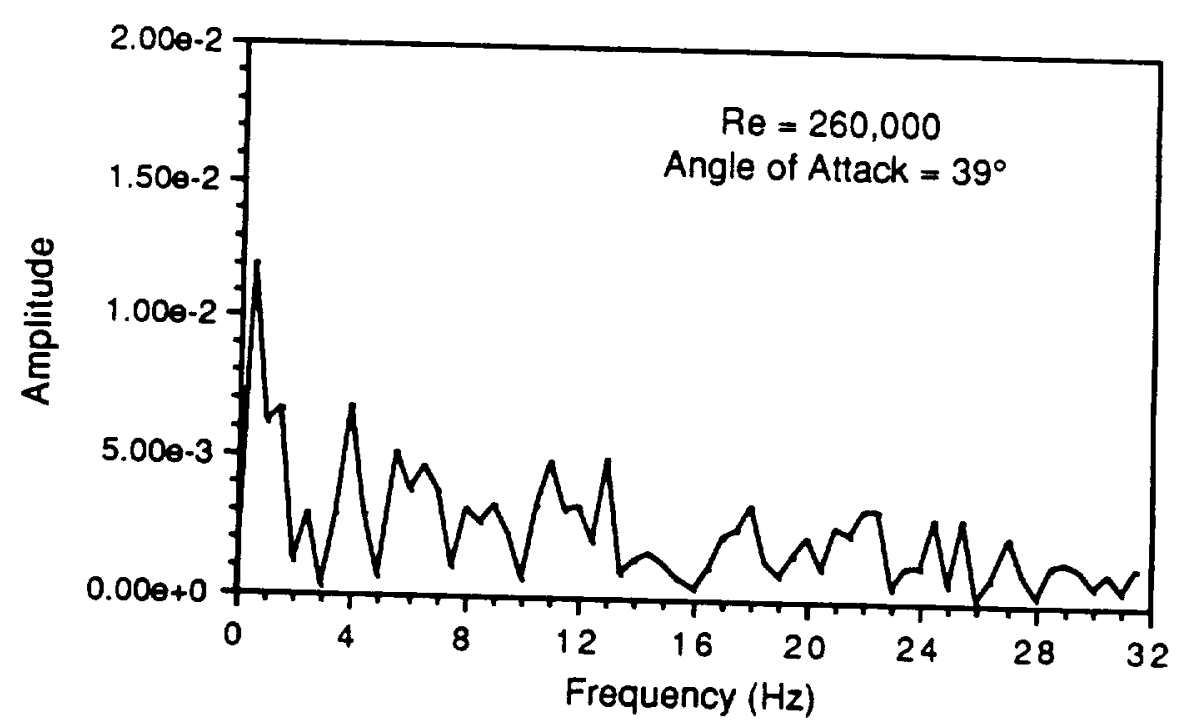

Figure 4.5b: Discrete Fourier Transform of the Variation in Chordwise Breakdown Location from Mean Position at an Angle of Attack of $39^{\circ} . U=30 \mathrm{ft} / \mathrm{s}, R \theta=260,000$. 


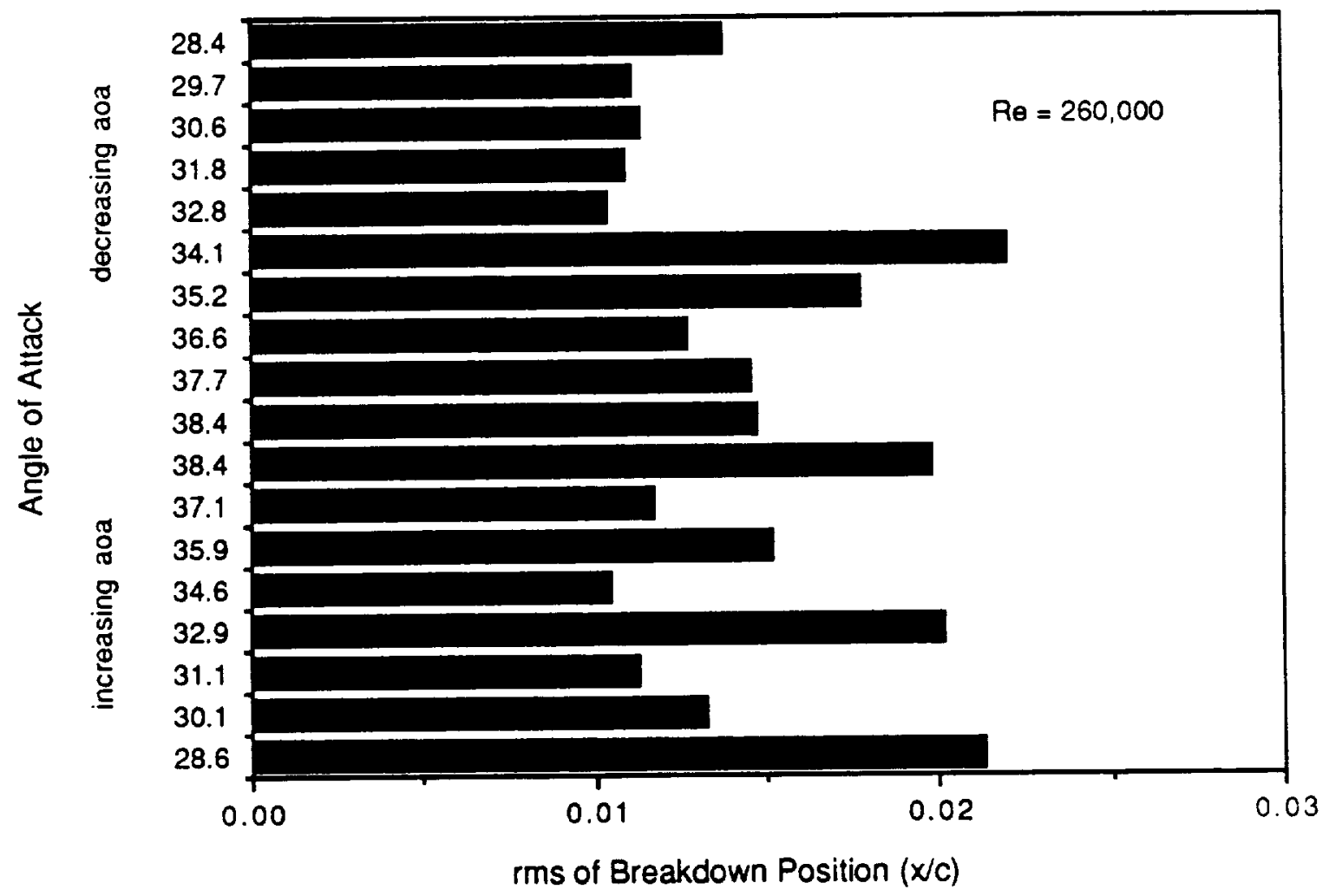

Figure 4.6: RMS of the Chordwise Breakdown Location at Static Angles of Attack throughout the Range of Pitching Motion. Angle of Attack Range $=29^{\circ}$ to $39^{\circ}, U=30^{\circ} \mathrm{tt} / \mathrm{s}, R \theta=260,000$. 

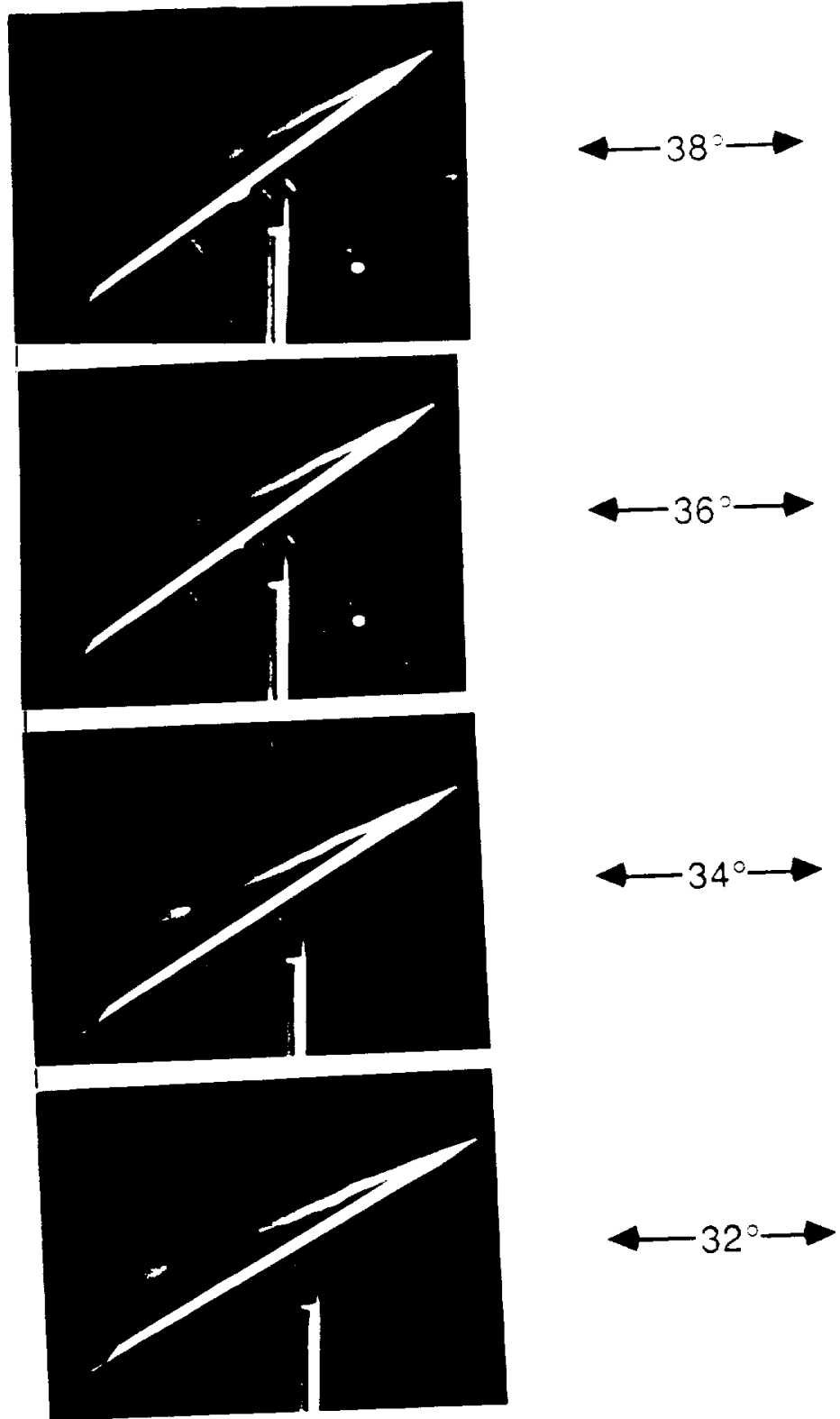
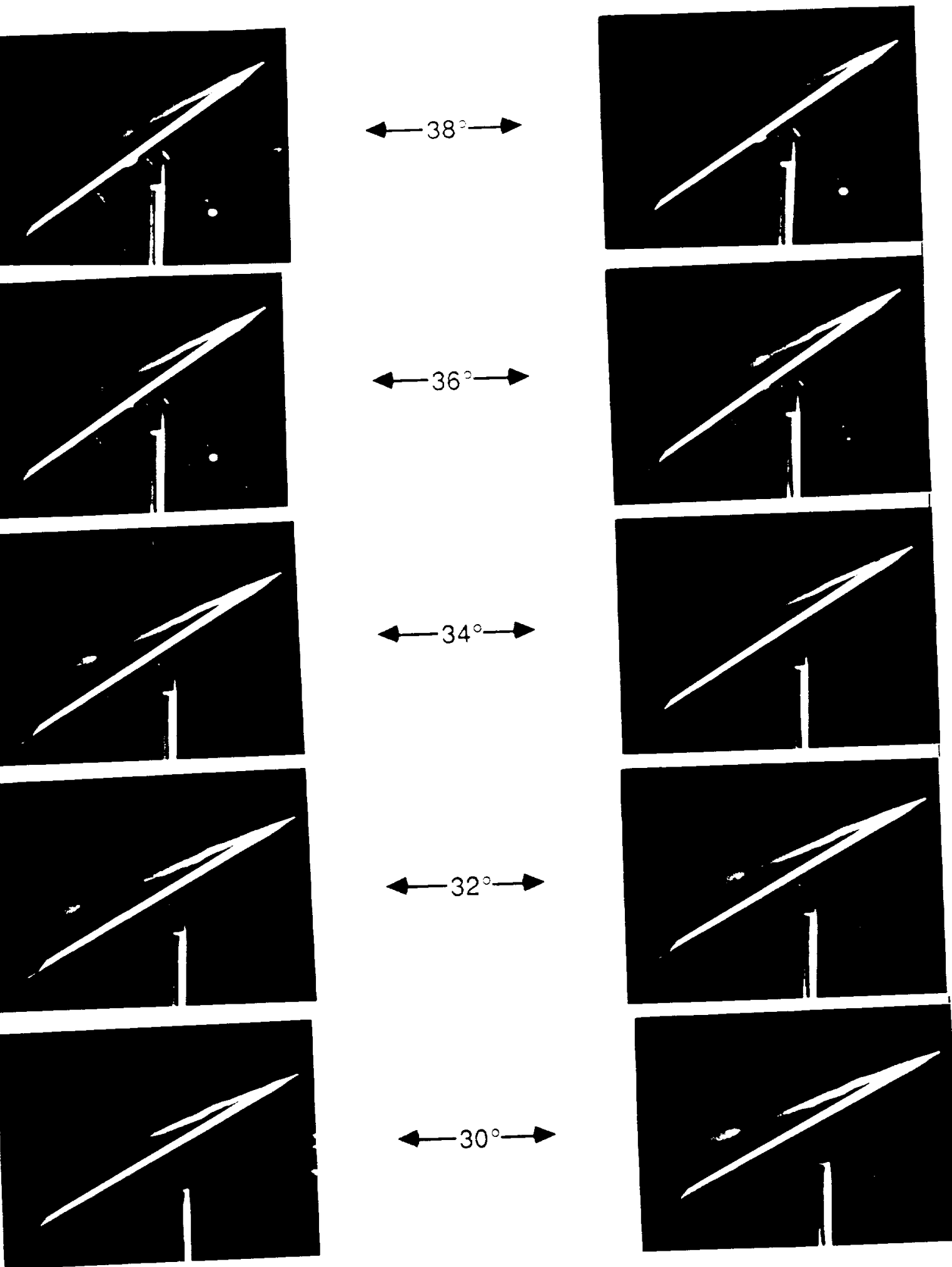

$\alpha$ Increasing

(a-e)

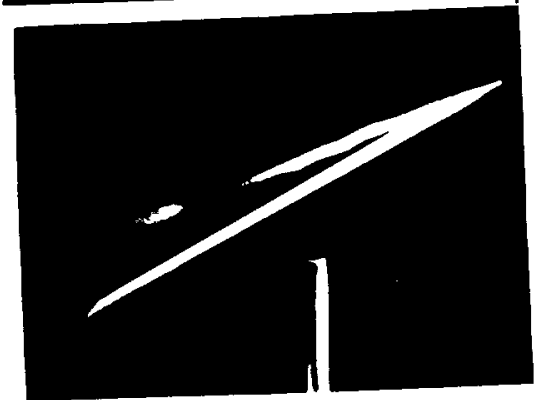

$\alpha$ Decreasing

$(f-j)$

Figure 4.7a-j: Sequence of Photographs made from $16 \mathrm{~mm}$ Movie Film which Illustrates how the Chordwise Breakdown Location varies throughout a Pitching Cycle. Angle of Attack Range $=29^{\circ}$ to $39^{\circ}$ 


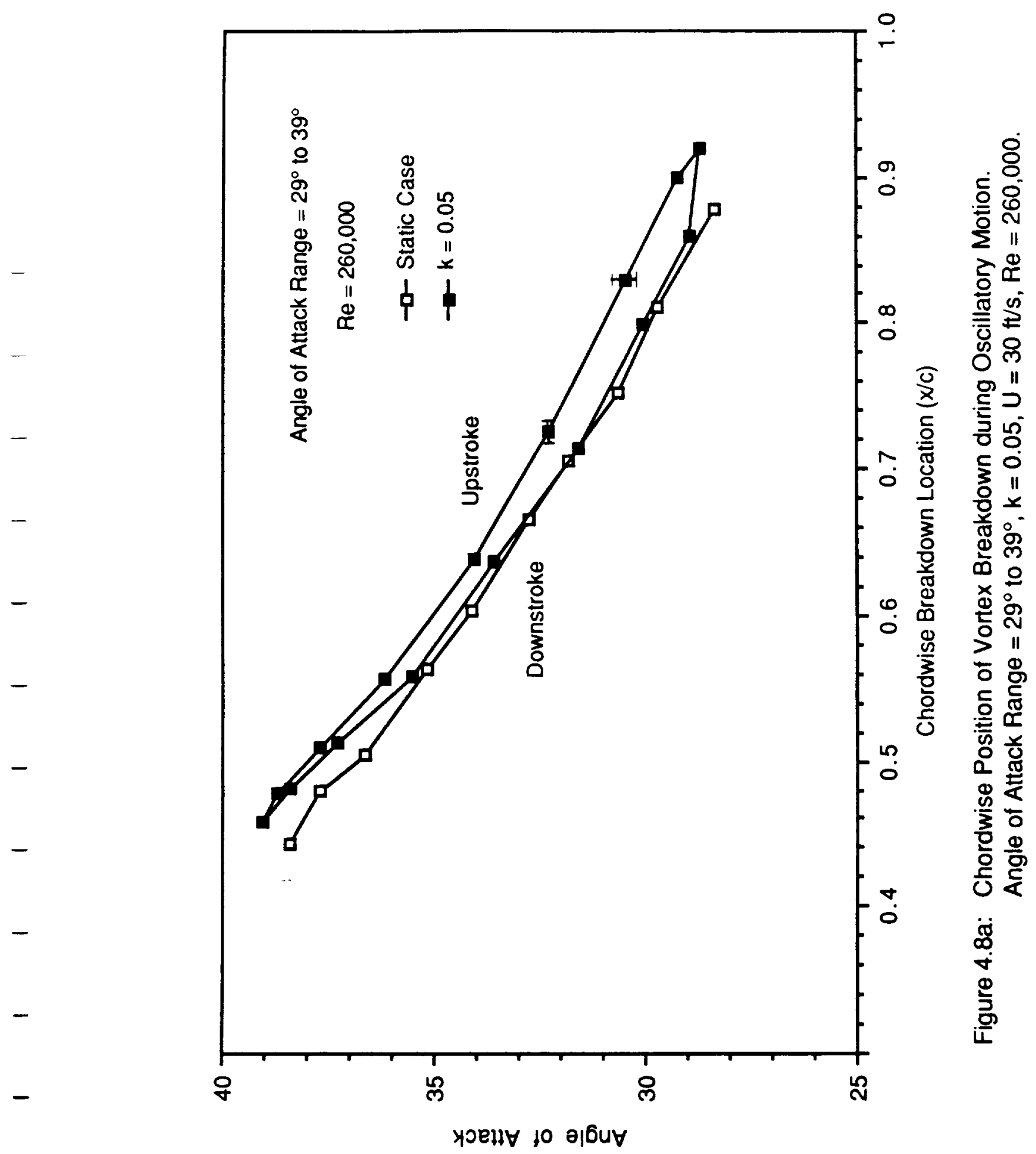




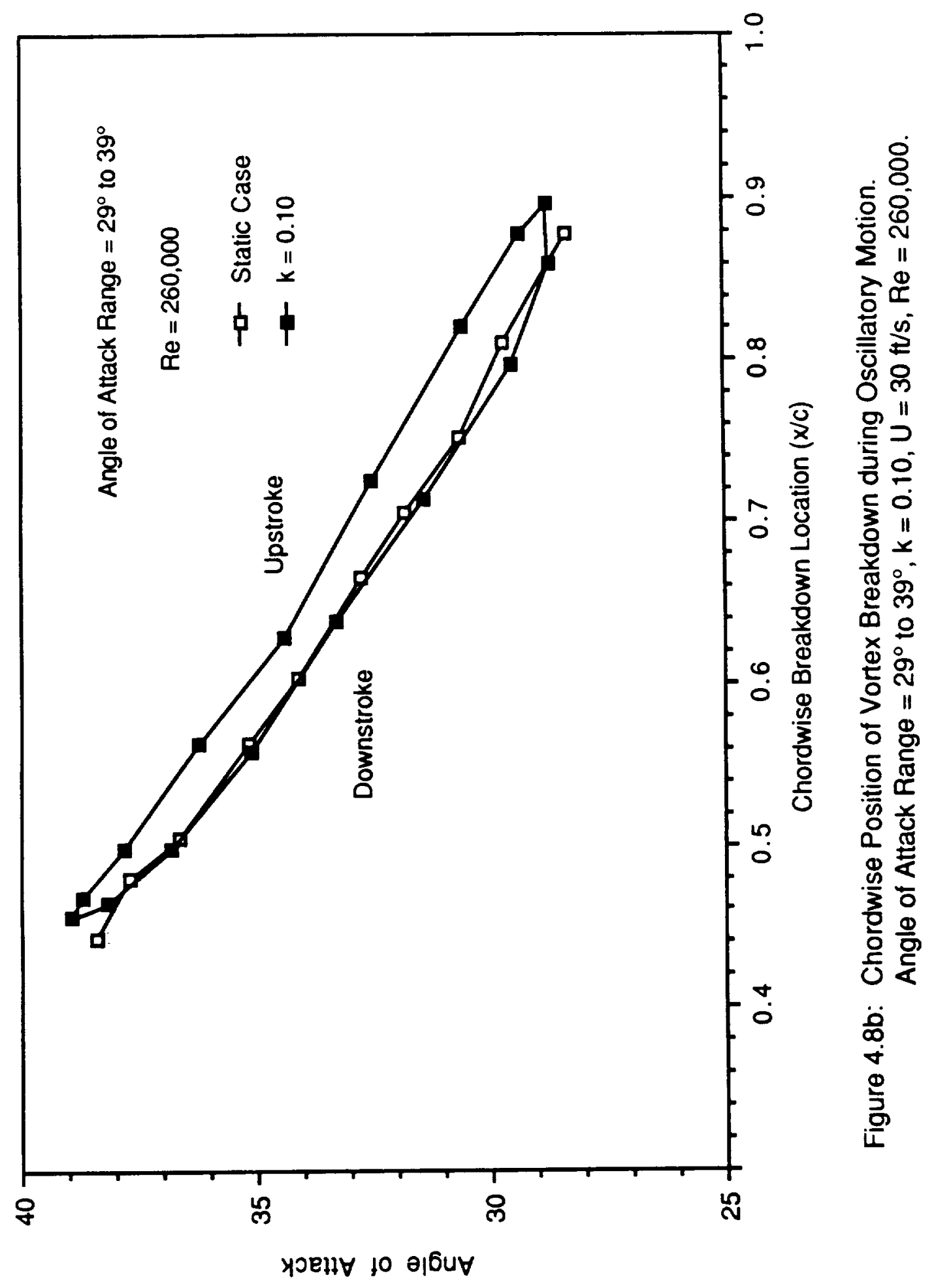




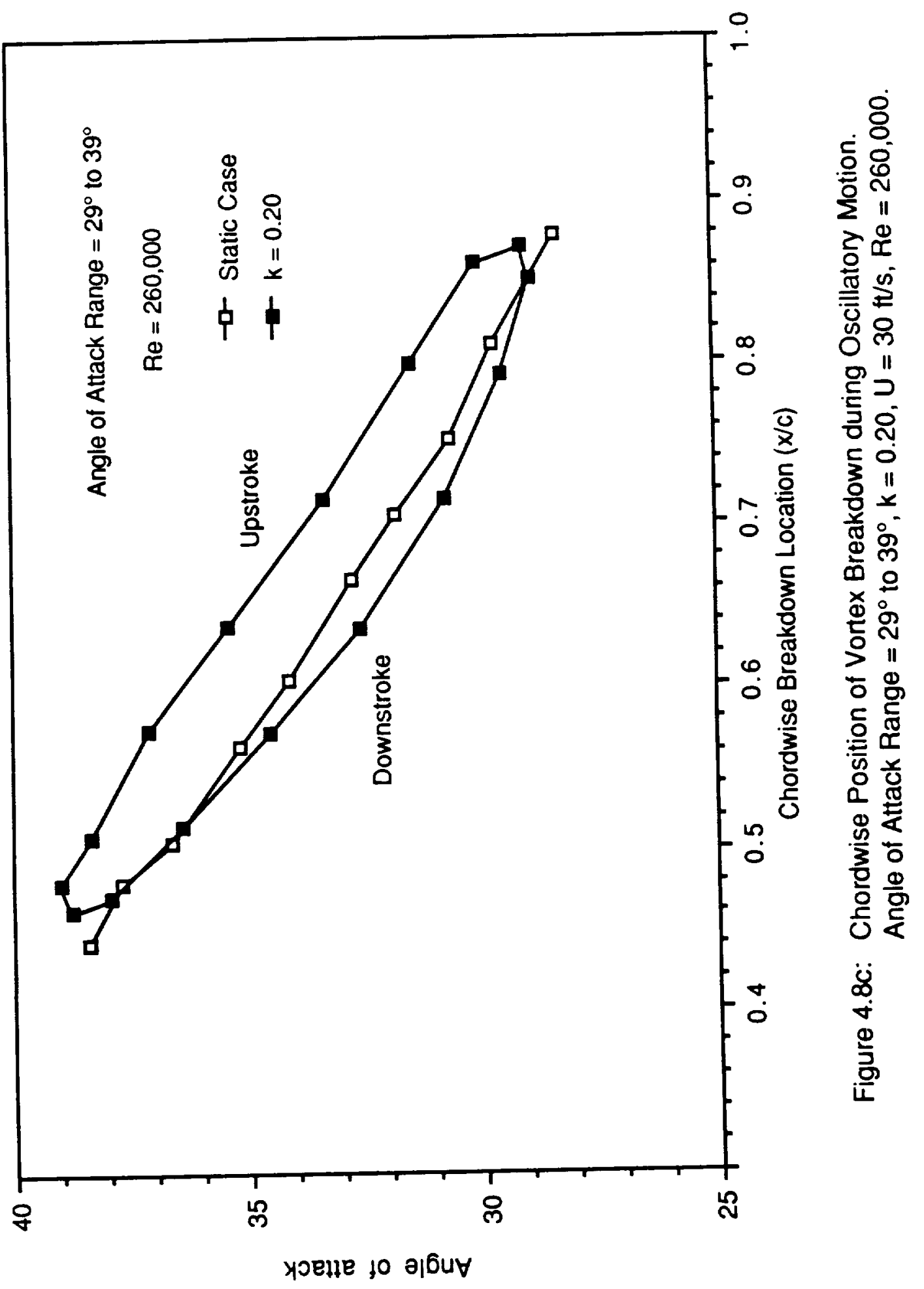




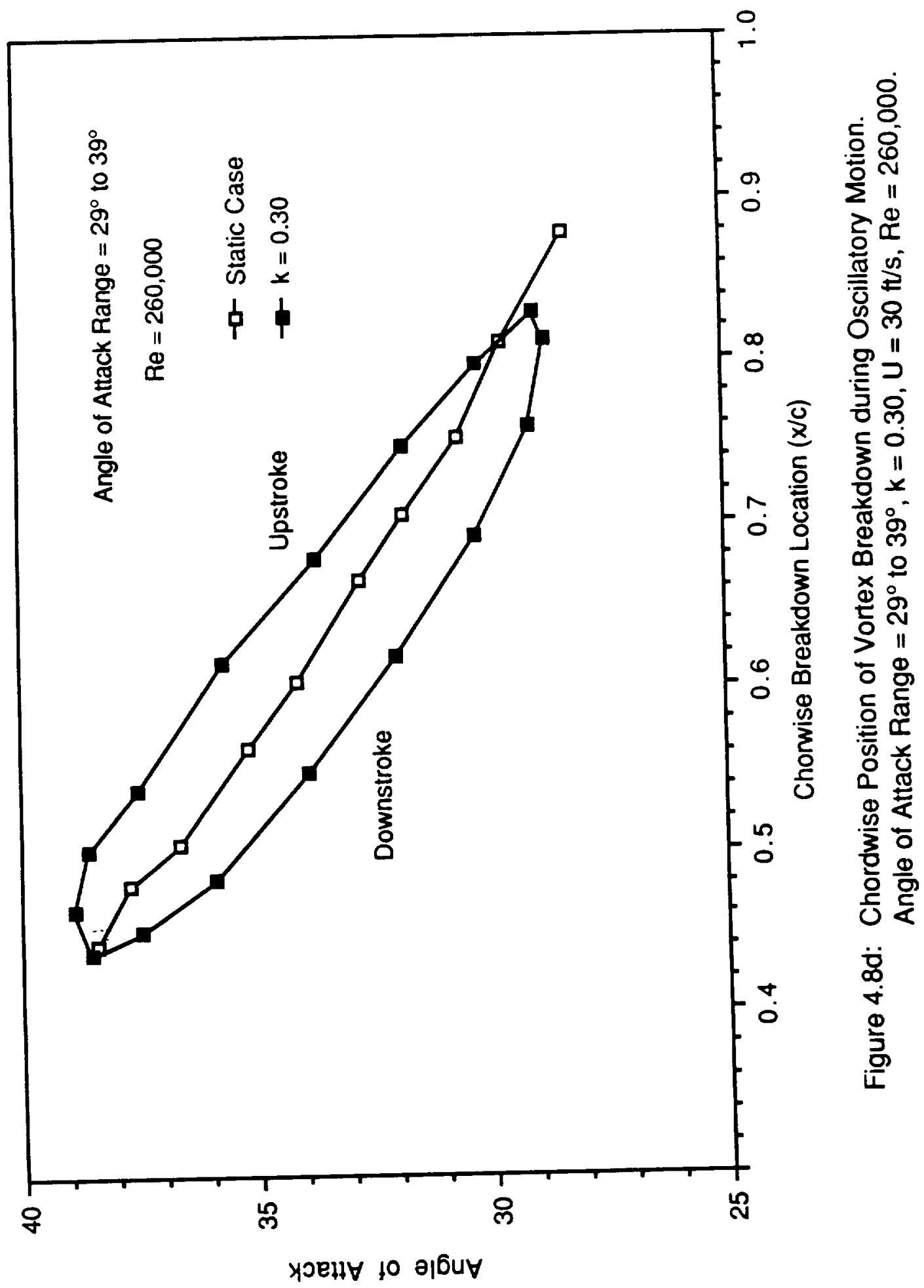




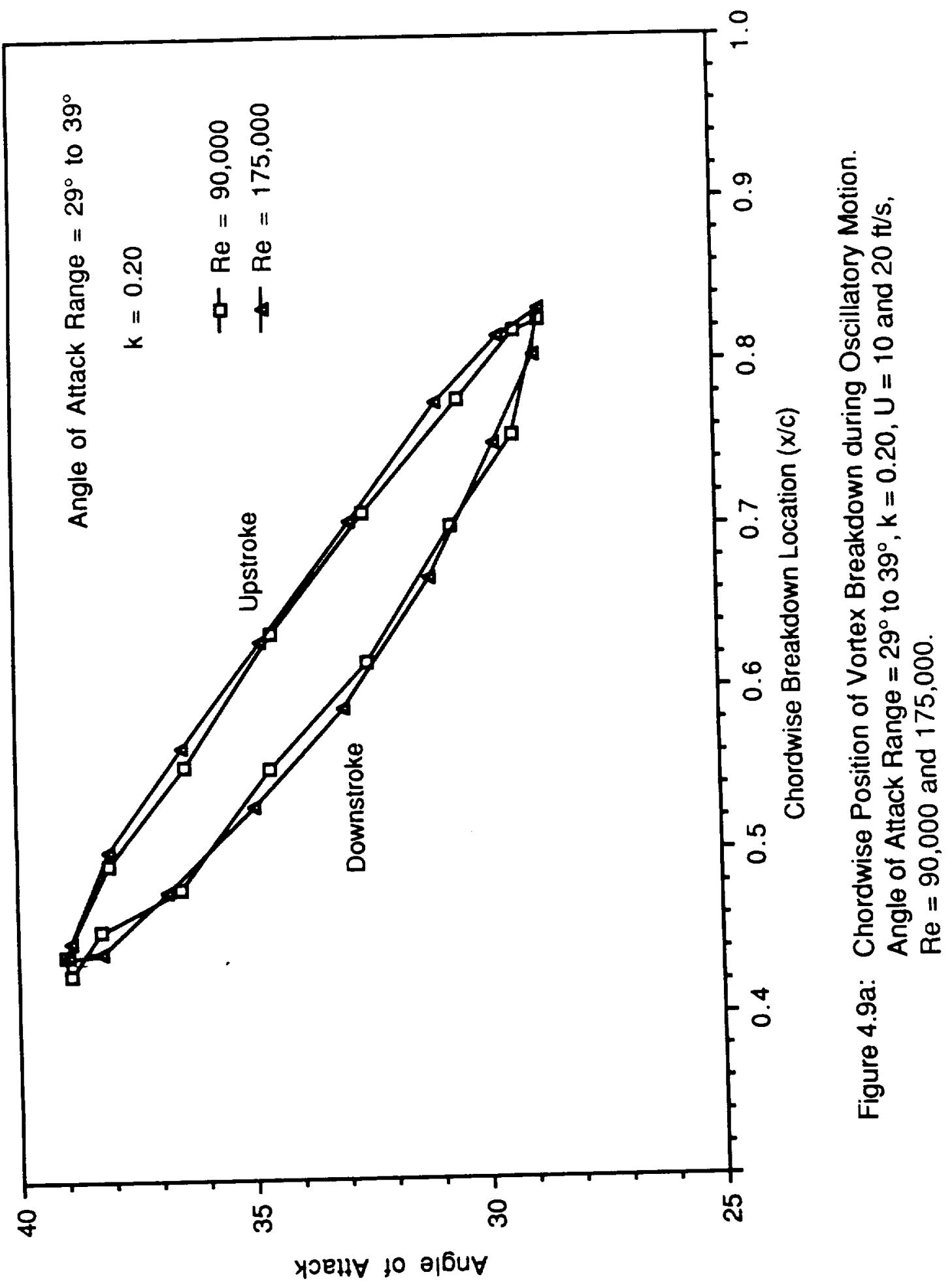




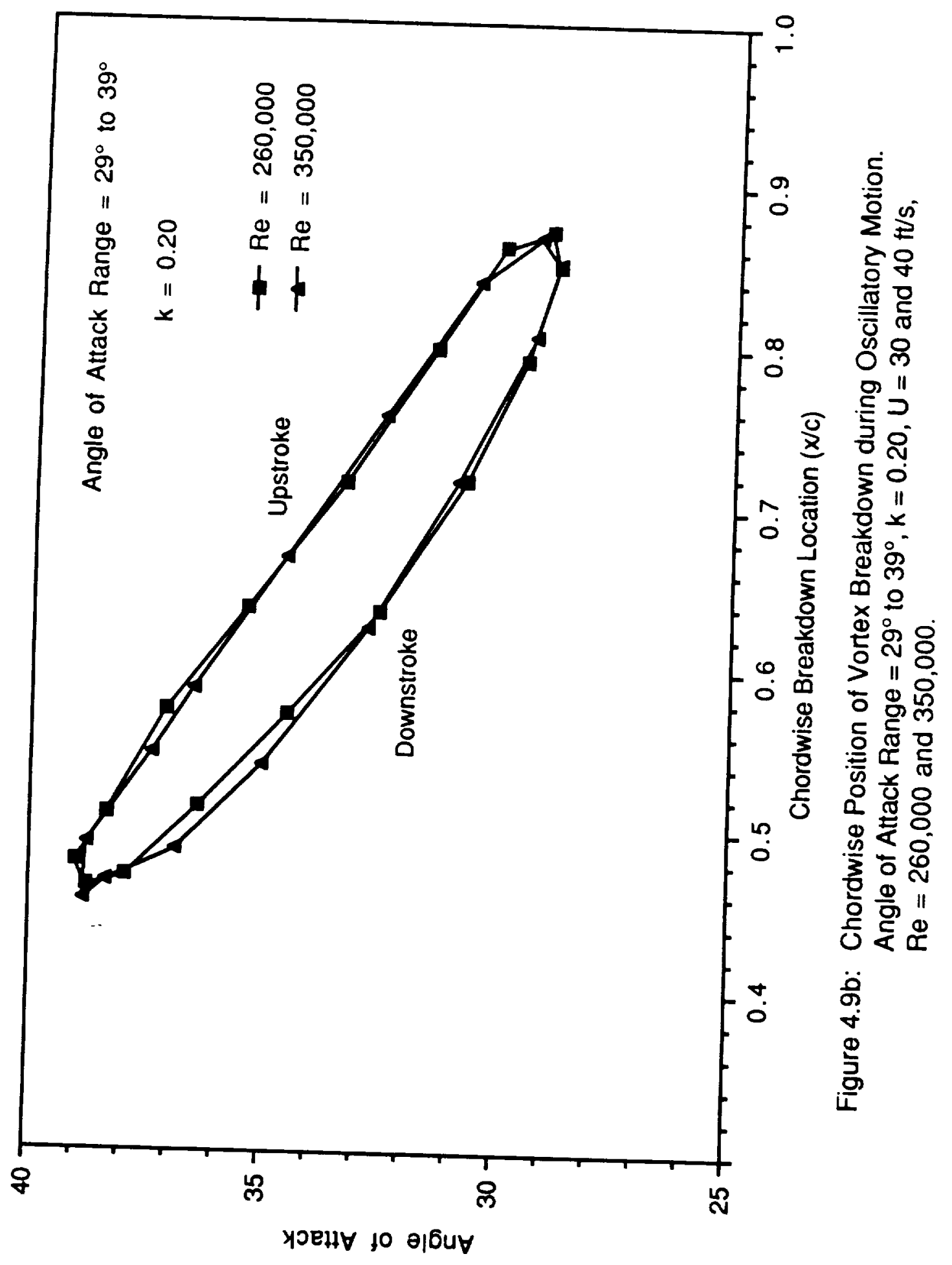




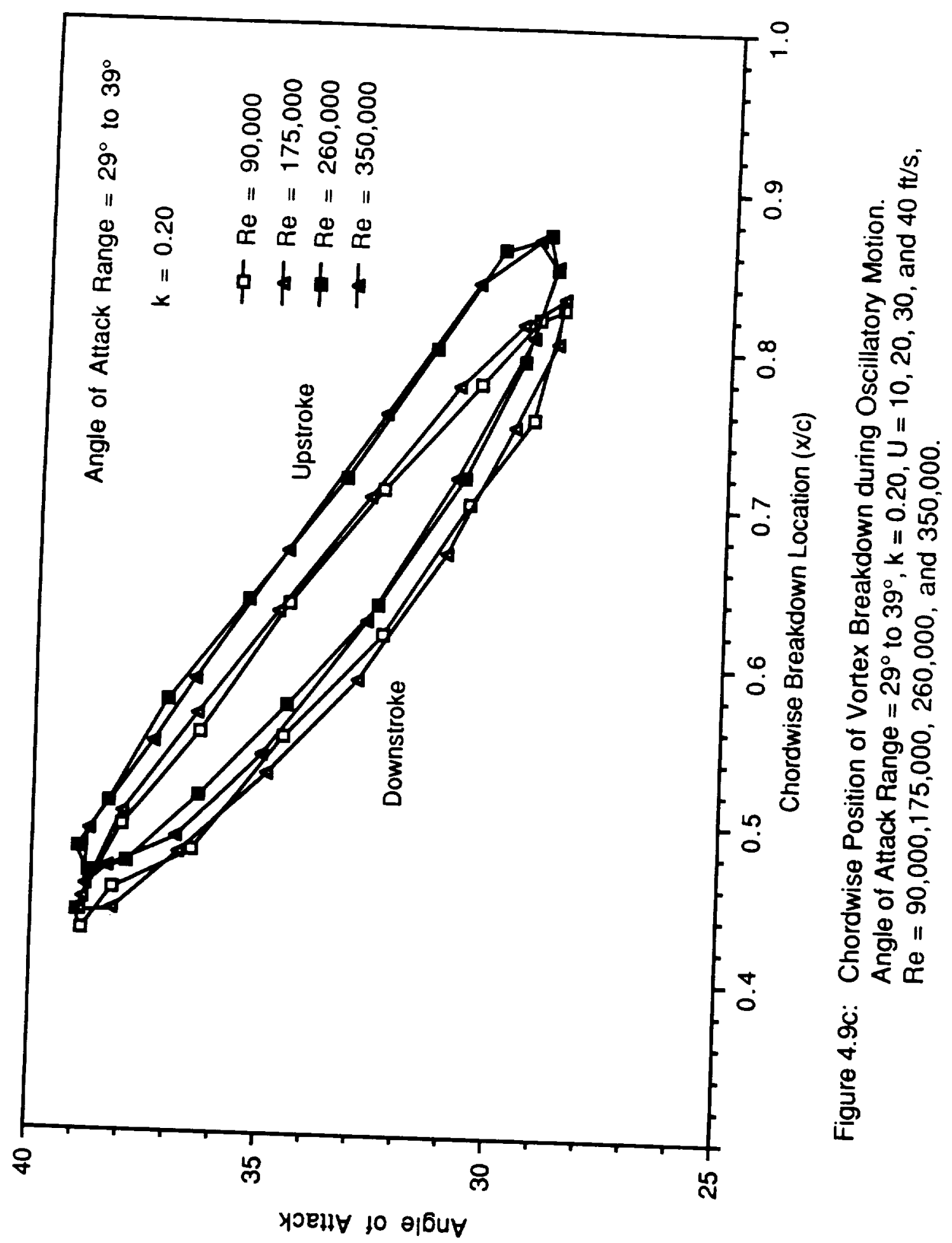




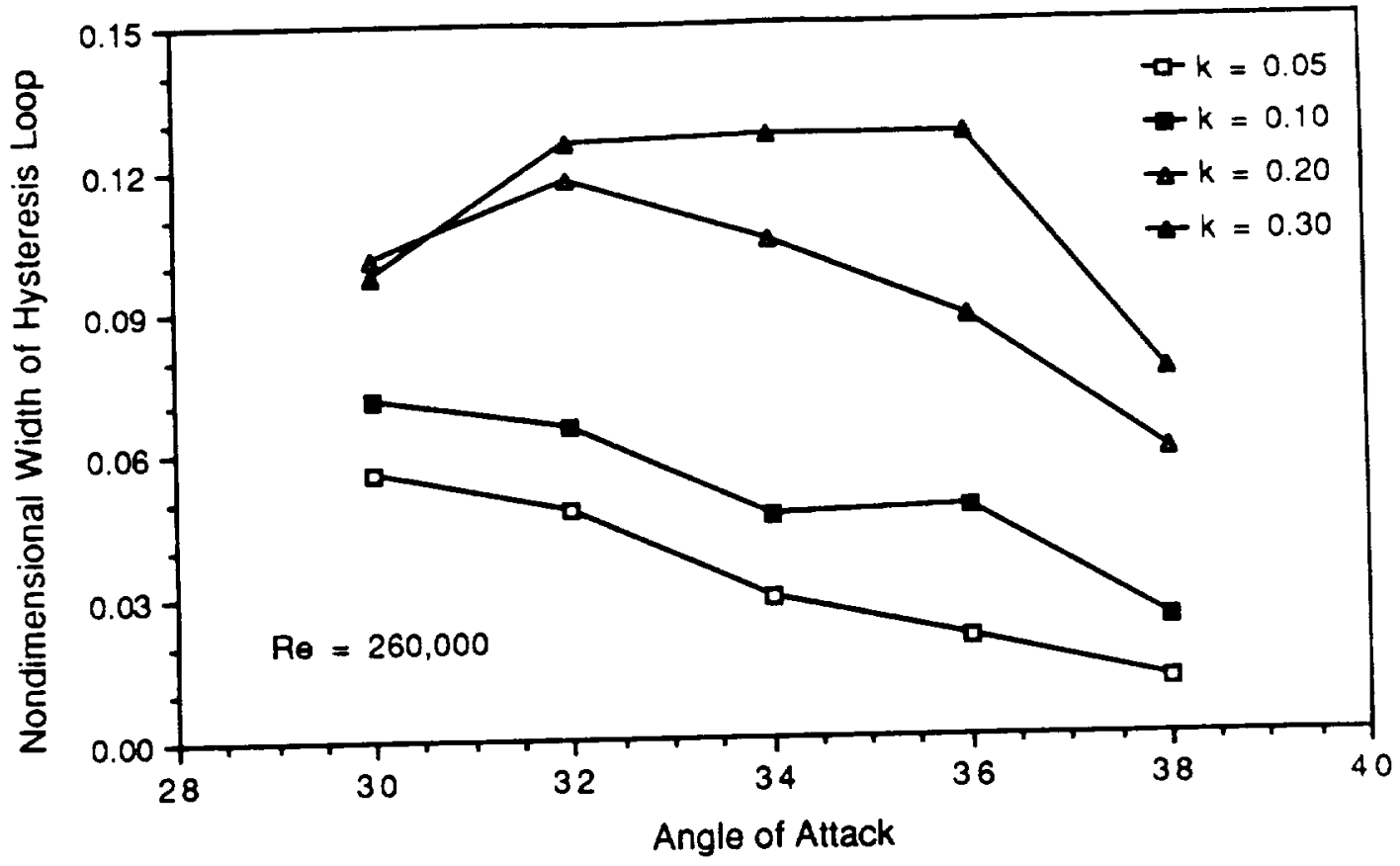

Figure 4.10a: Width of the Hysteresis Loops, presented in Figures 4.8a-d, over the Angle of Attack Range of $29^{\circ}$ to $39^{\circ}$ for Reduced Frequencies of $k=0.05,0.10,0.20$, and $0.30 . U=30 \mathrm{ft} / \mathrm{s}, R e=260,000$.

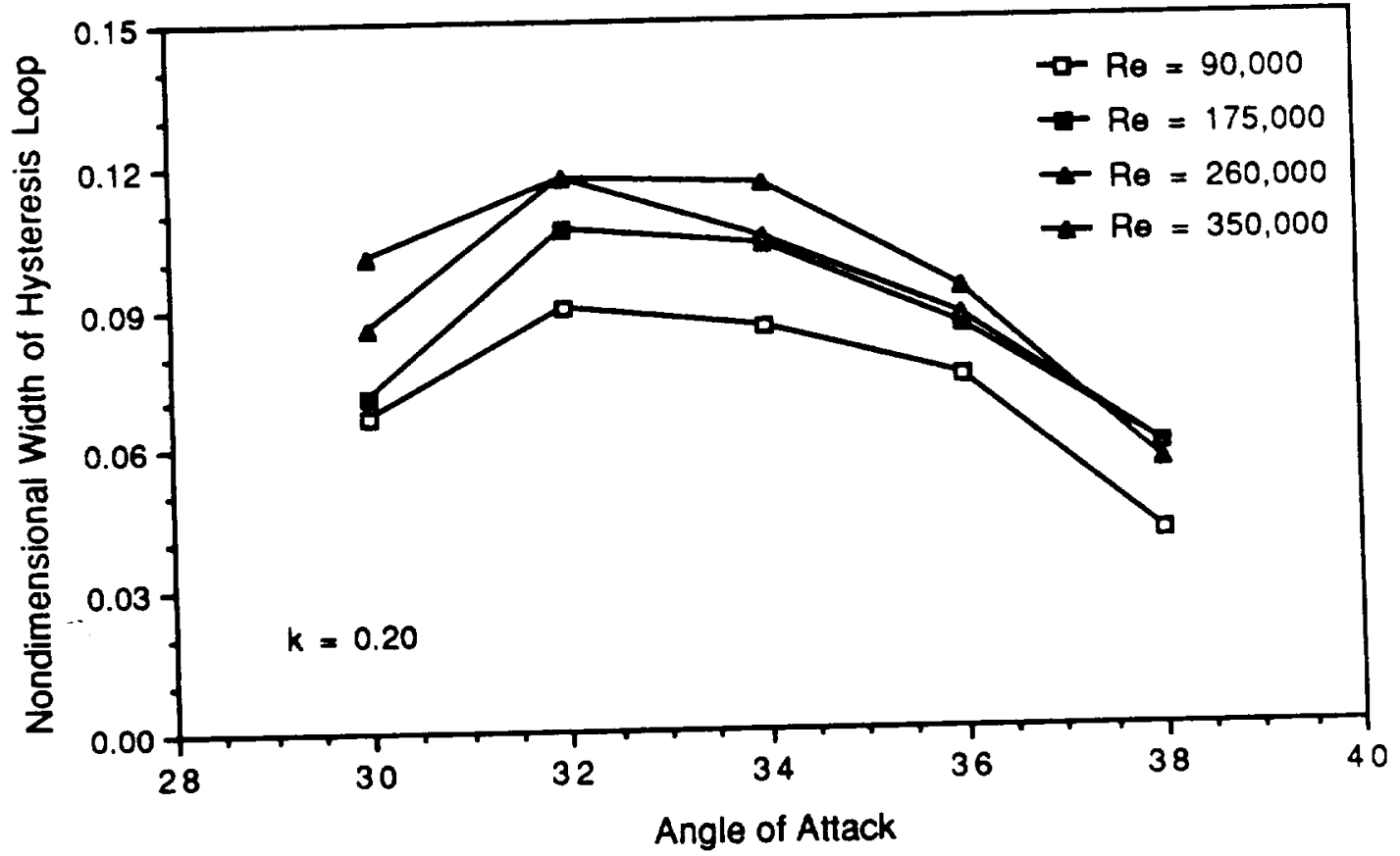

Figure 4.10b: Width of the Hysteresis Loops, presented in Figures 4.9a-c, over the Angle of Attack Range of $29^{\circ}$ to $39^{\circ}$ for Reynolds Number of $R_{\theta}=90,000,175,000,260,000$ and $350,000 . k=0.20$, $U=10,20,30$, and $40 \mathrm{ft} / \mathrm{s}$. 


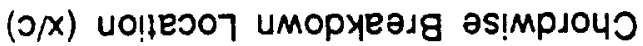
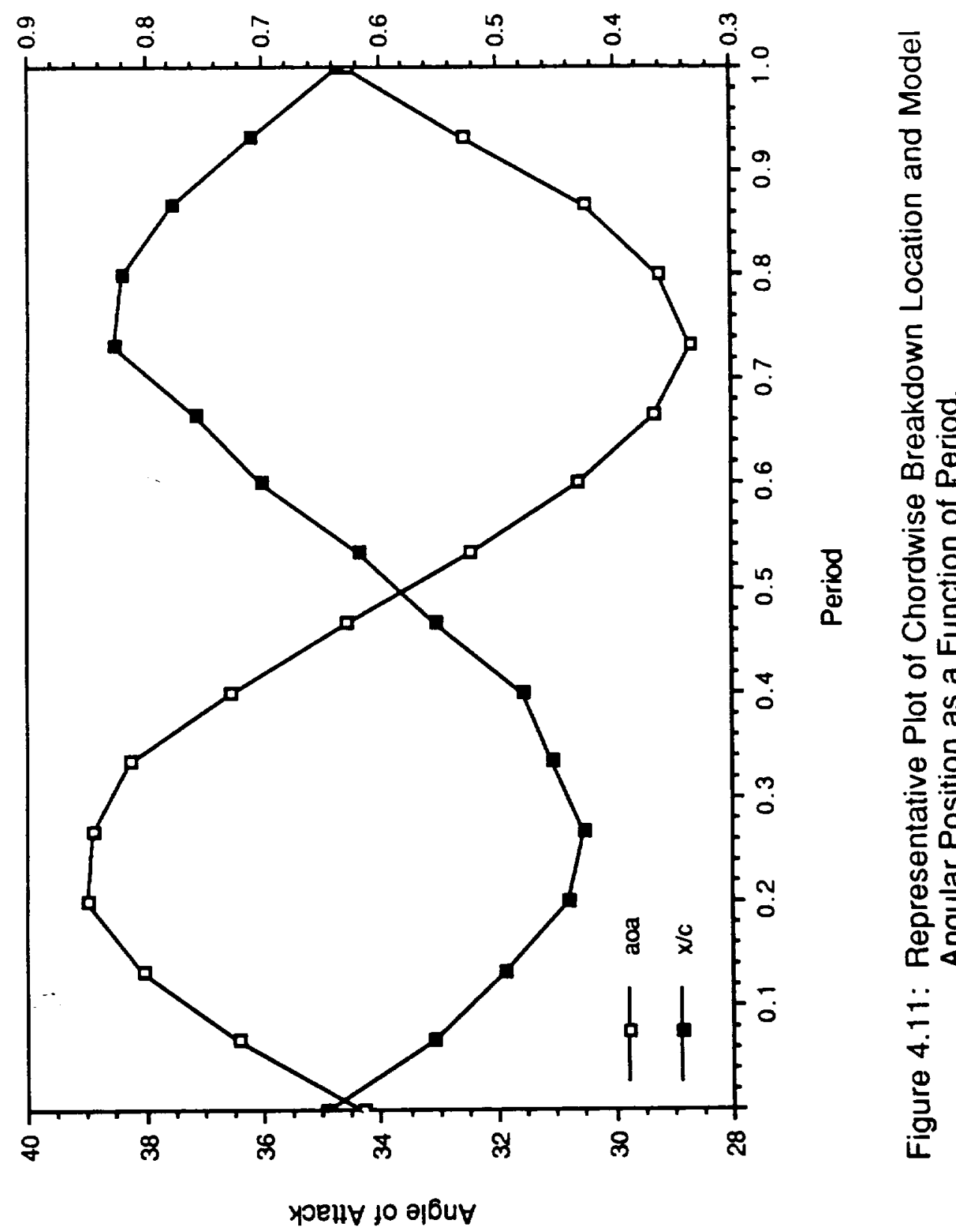

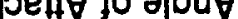




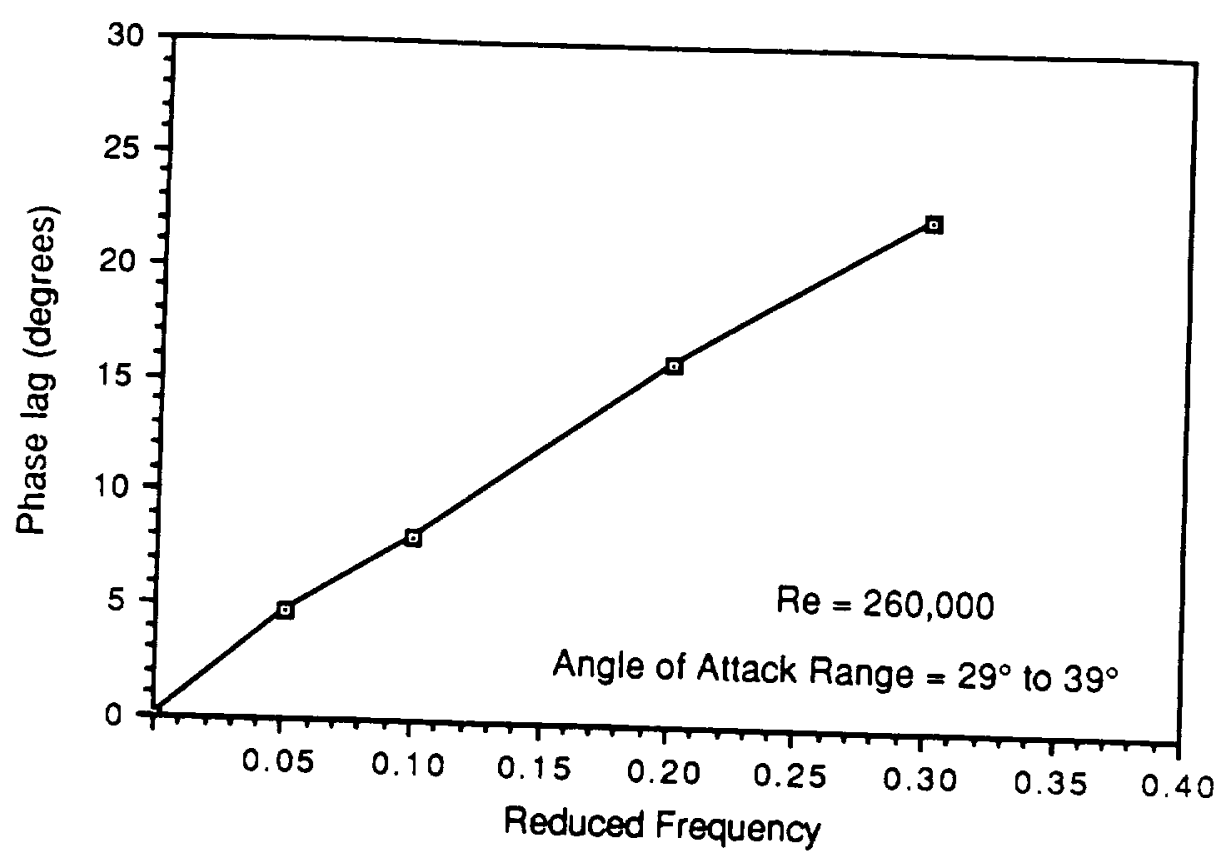

Figure 4.12: Phase Lag of the Vortex Flow as a Function of Reduced Frequency for a Reynolds number of 260,000 . Angle of Attack Range $=29^{\circ}$
to $39^{\circ}, U=30 \mathrm{ft} / \mathrm{s}$.

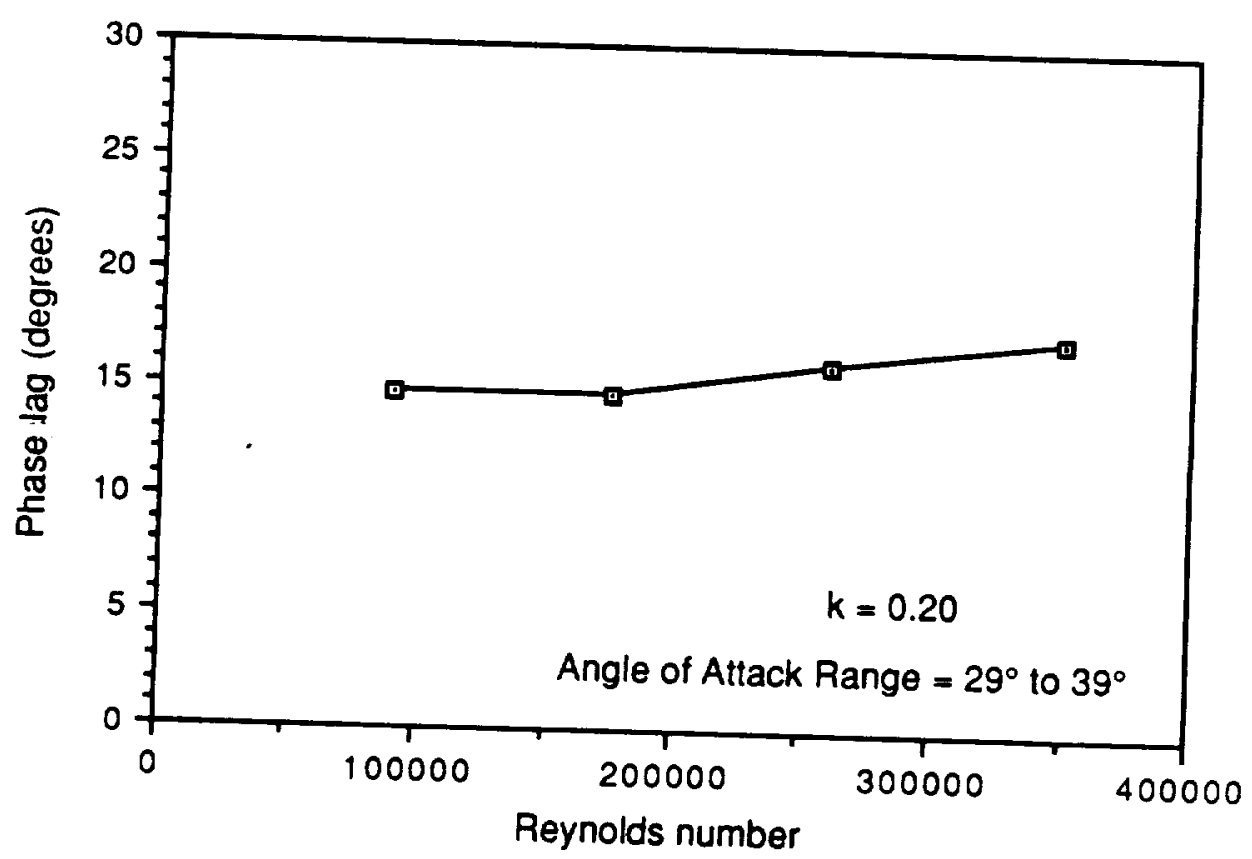

Figure 4.13: Phase Lag of the Vortex Flow as a Function of Reynolds Number to $39^{\circ}$. 


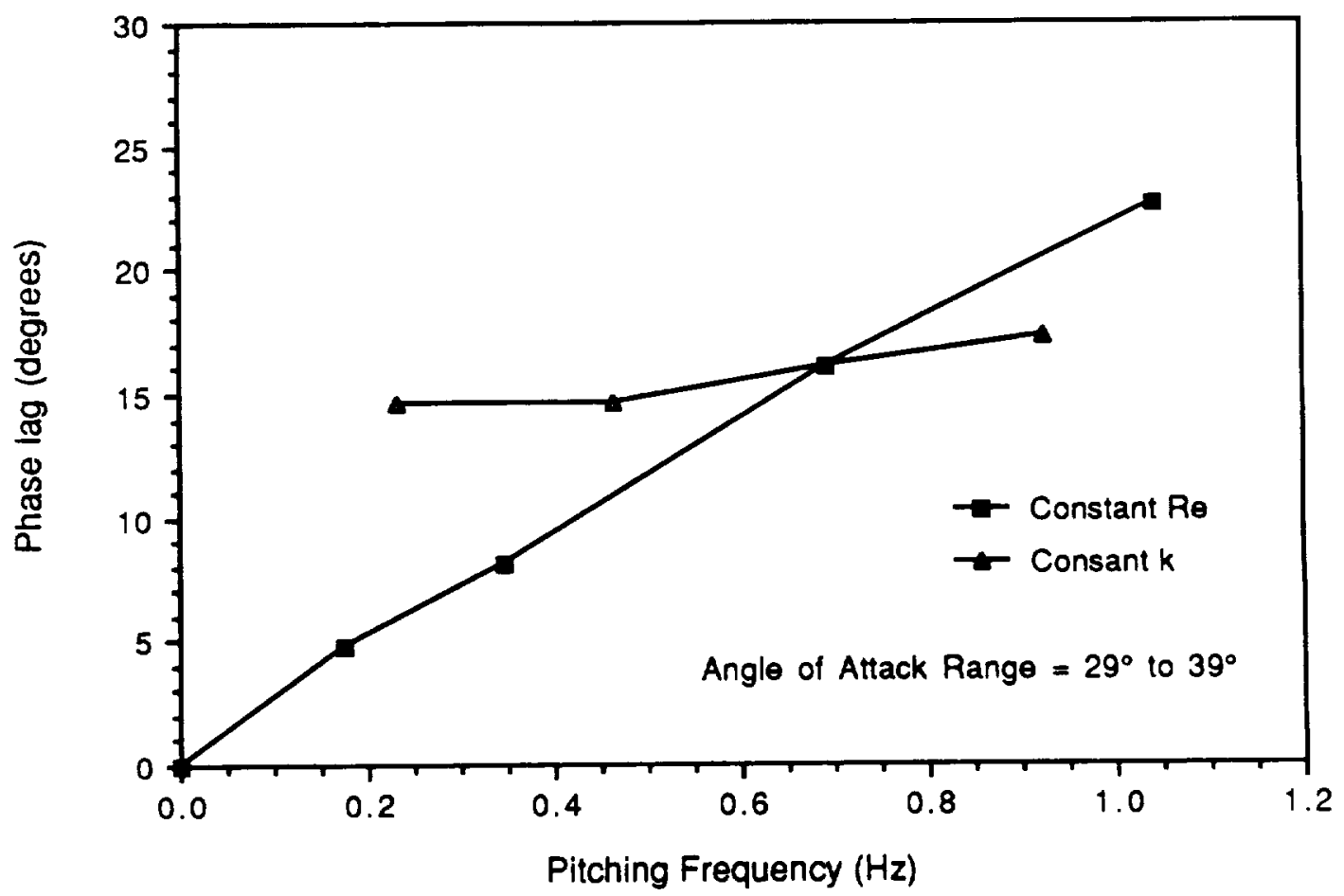

Figure 4.14: Phase Lag of the Vortex Flow as a Function of Pitching Frequency for a Reynolds Number of 260,000 and a Reduced Frequency of $k=0.20$. Angle of Attack Range $=29^{\circ}$ to $39^{\circ}$. 


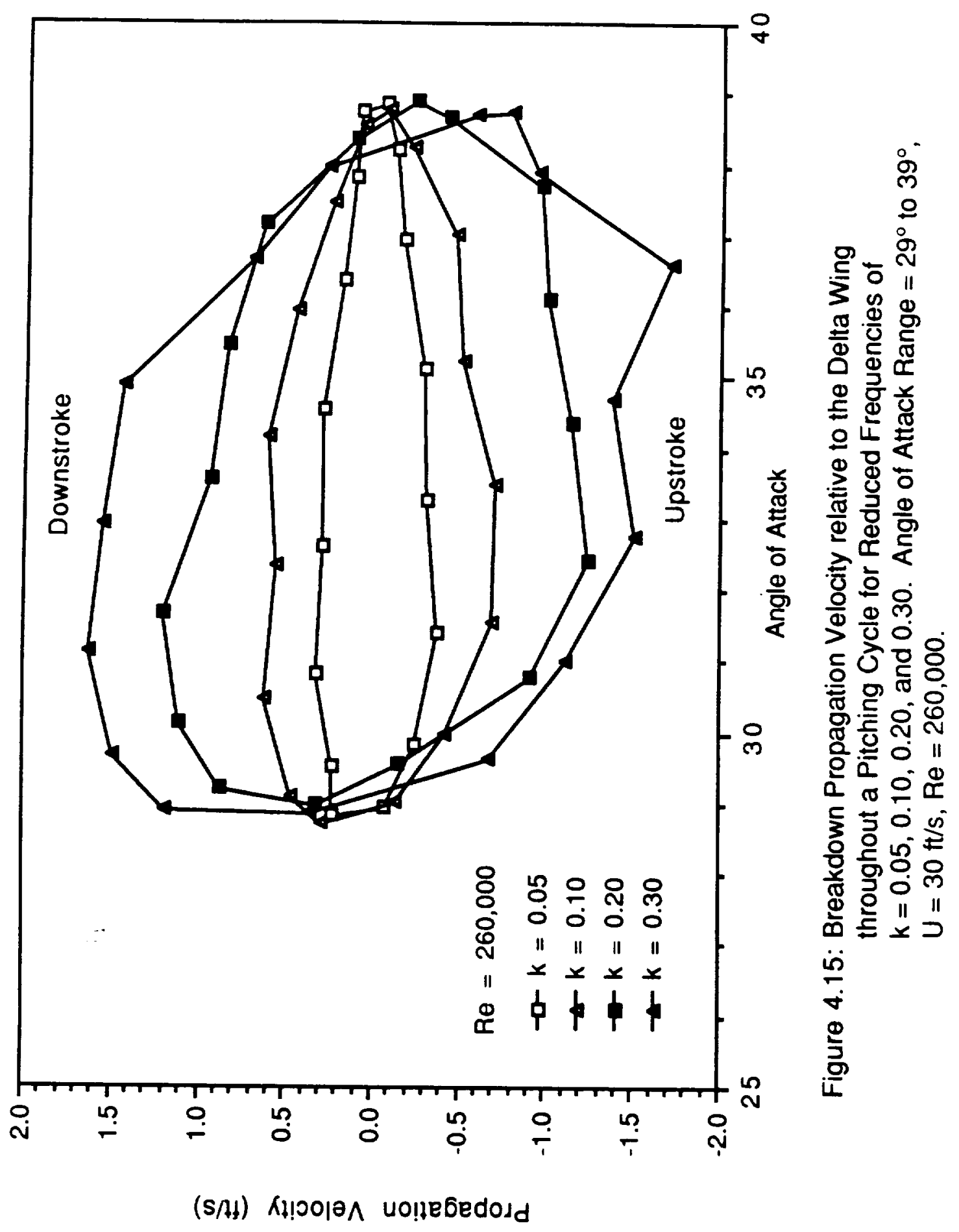




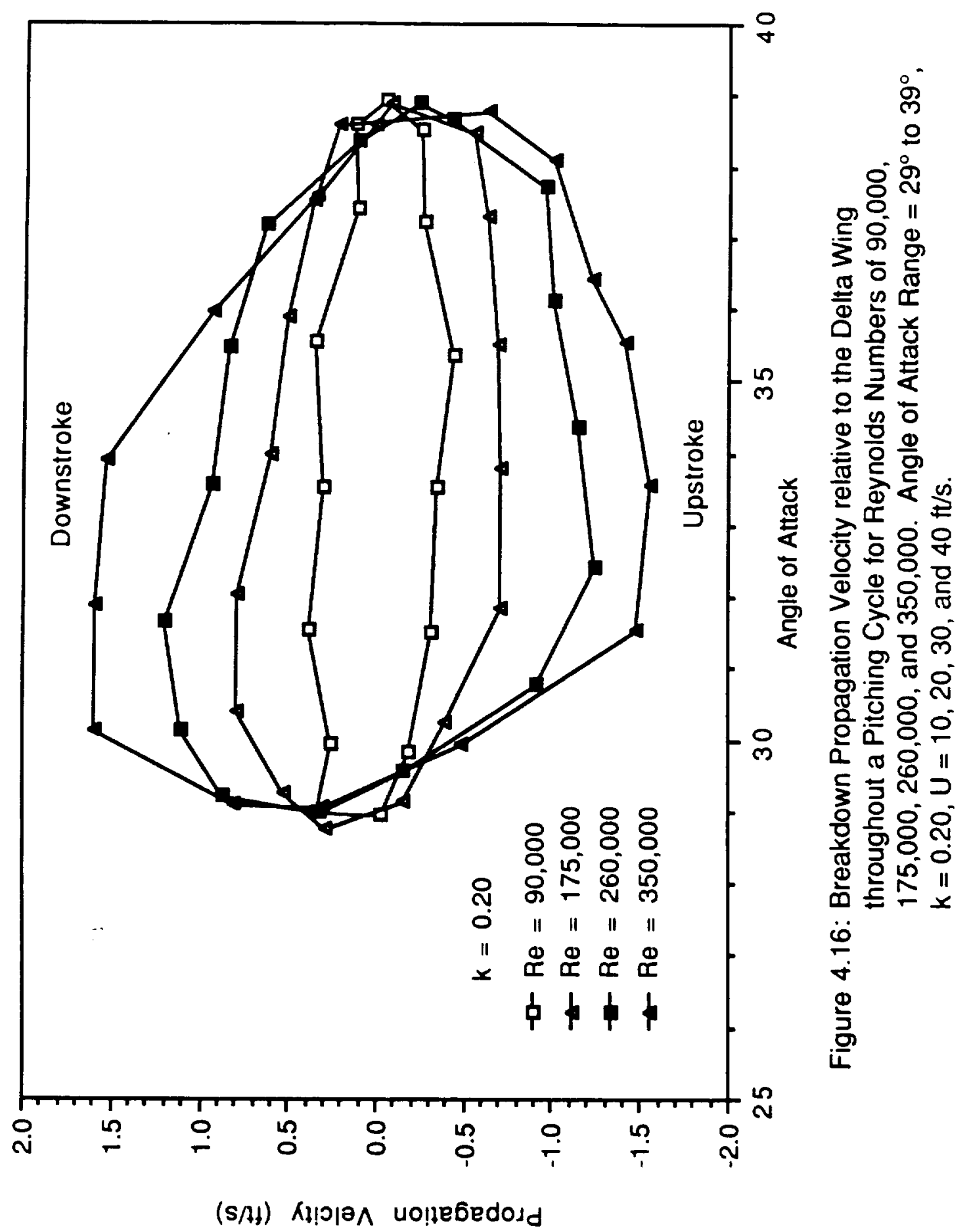




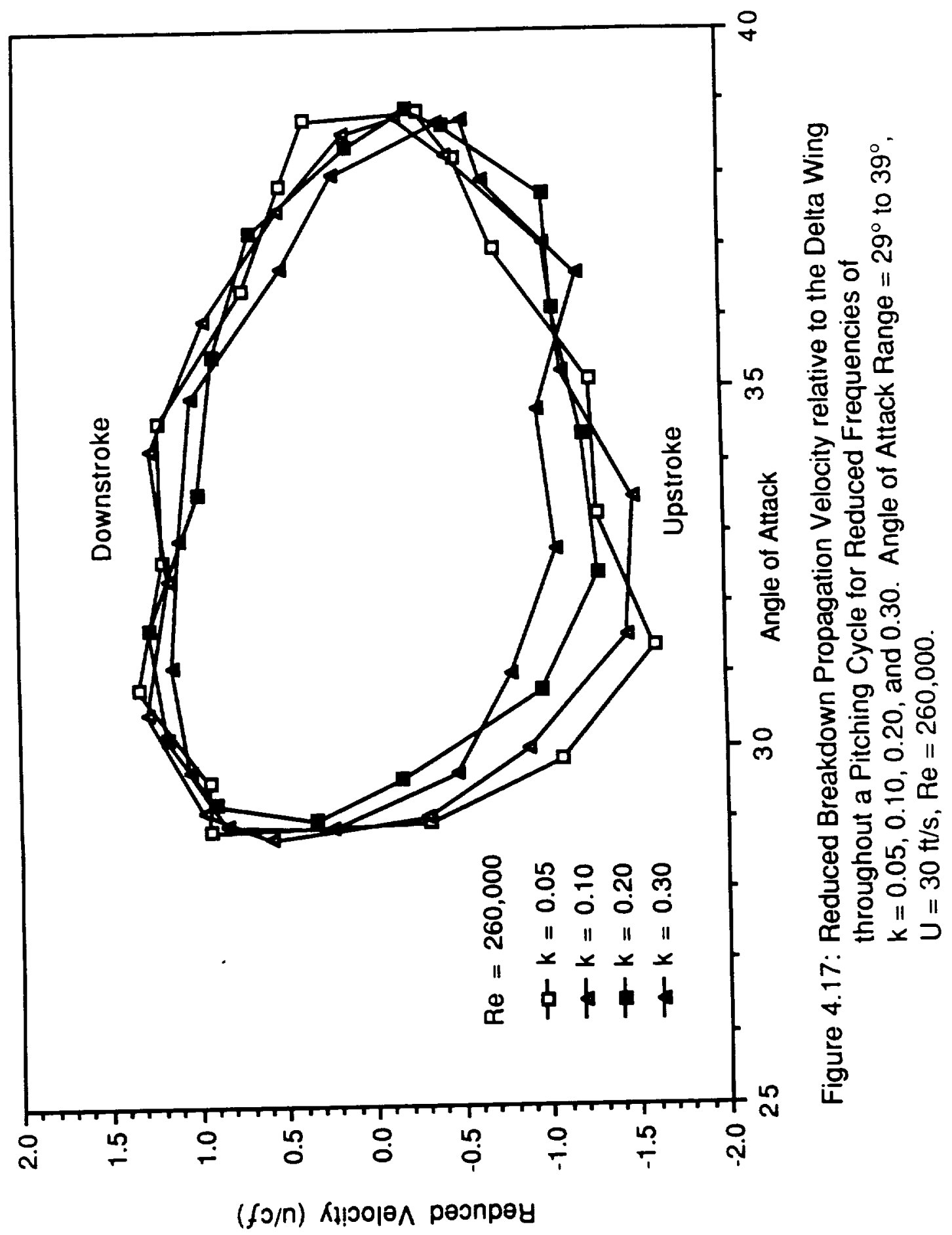




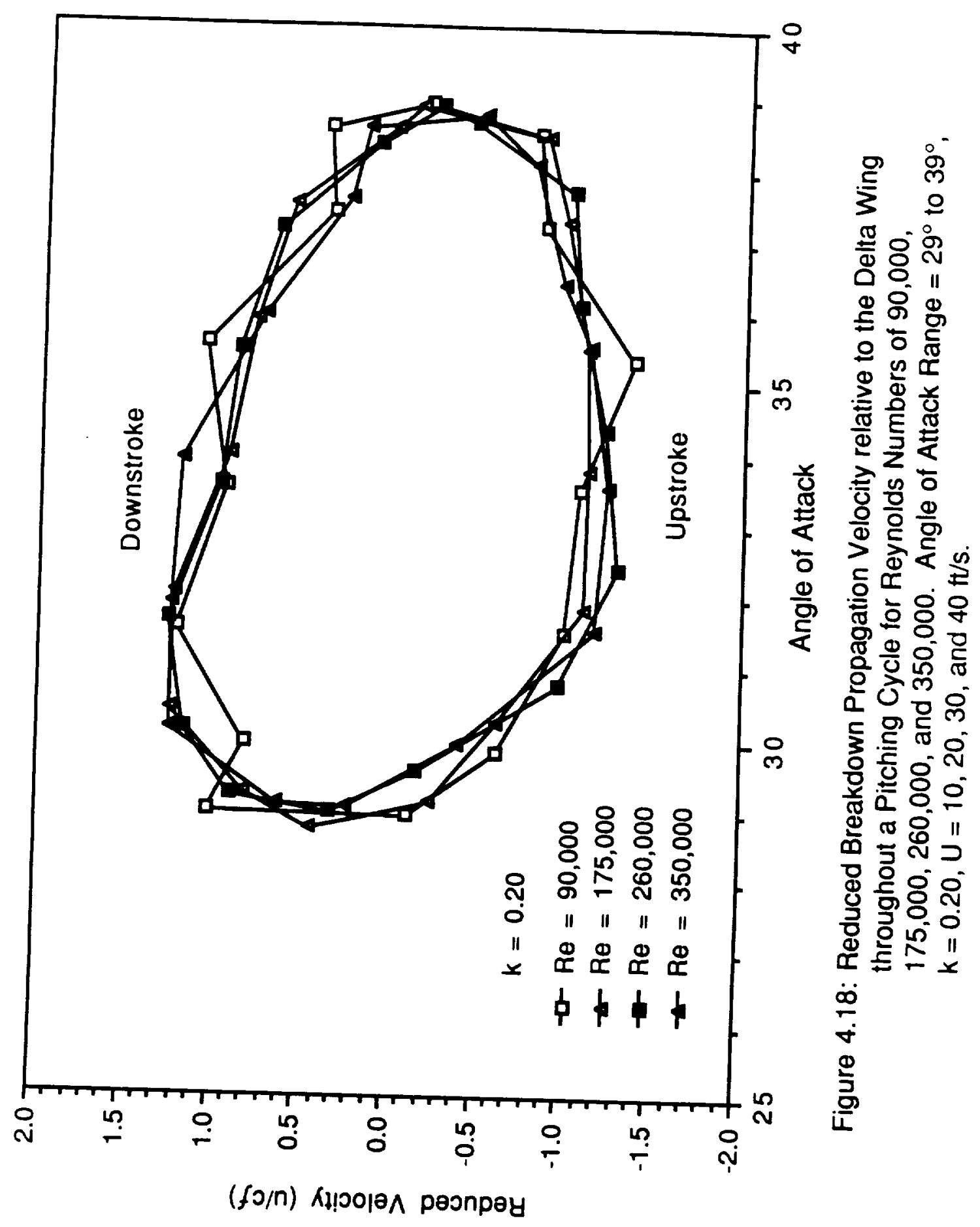




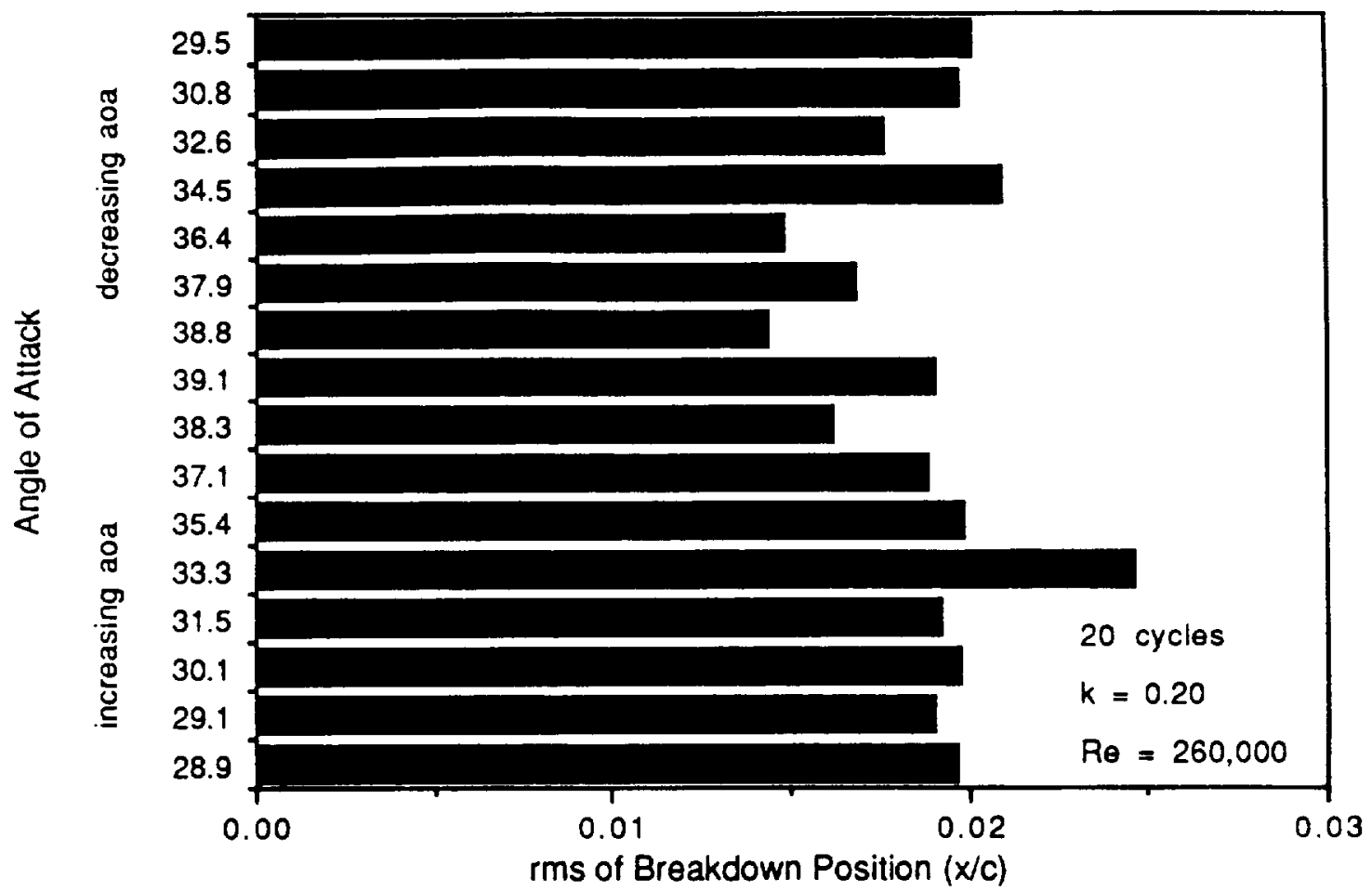

Figure 4.19a: RMS of the Chordwise Breakdown Location at Various Angles of Attack throughout a Pitching Cycle. Angle of Attack Range $=29^{\circ}$ to $39^{\circ}, k=0.20, U=30 \mathrm{ft} / \mathrm{s}, R \theta=260,000$.

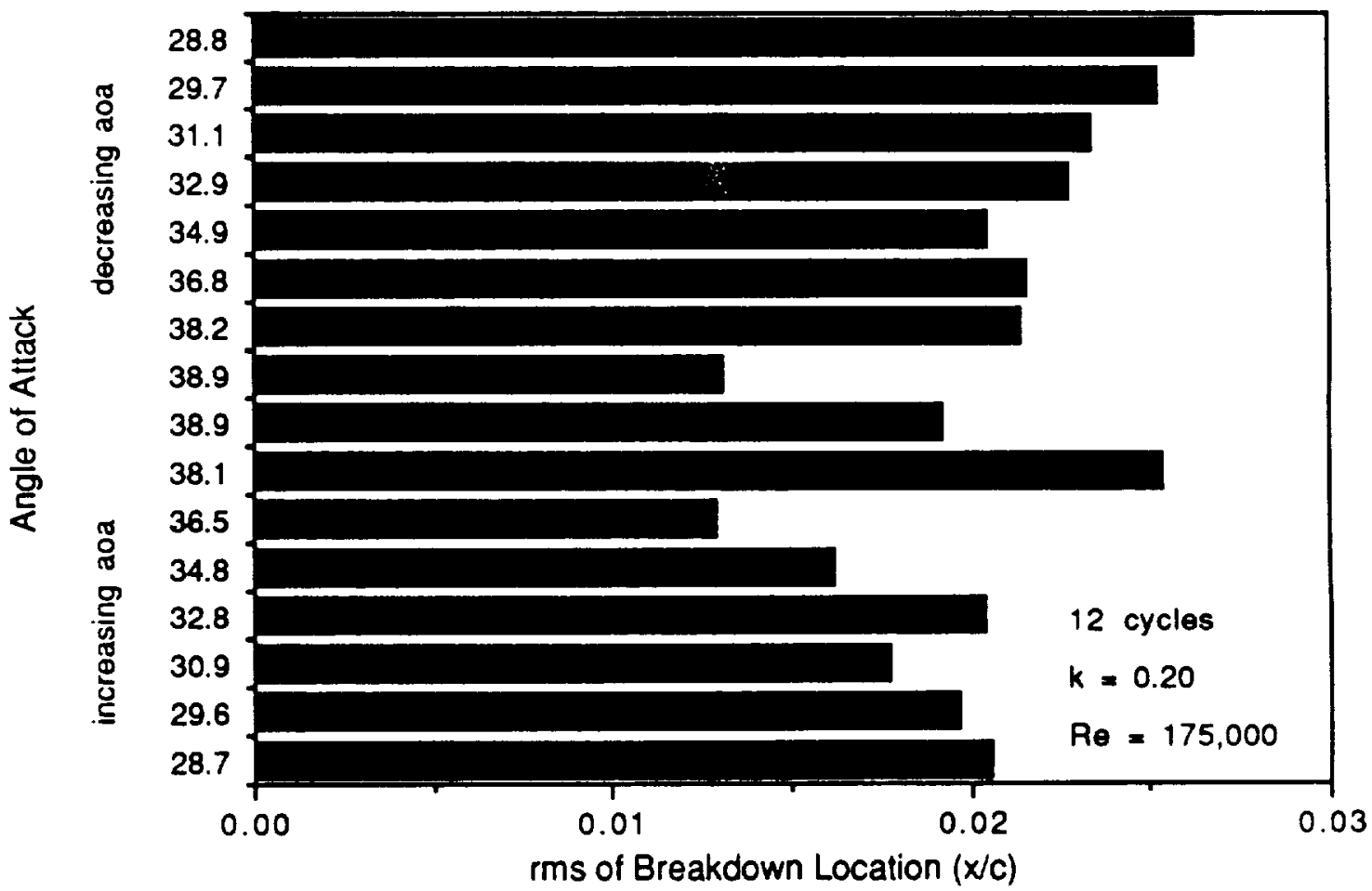

Figure 4.19b: RMS of the Chordwise Breakdown Location at Various Angles of Attack throughout a Pitching Cycle. Angle of Attack Range $=29^{\circ}$ to $39^{\circ}, \mathrm{k}=0.20, \mathrm{U}=20 \mathrm{ft} / \mathrm{s}, \mathrm{Re}=175,000$. 


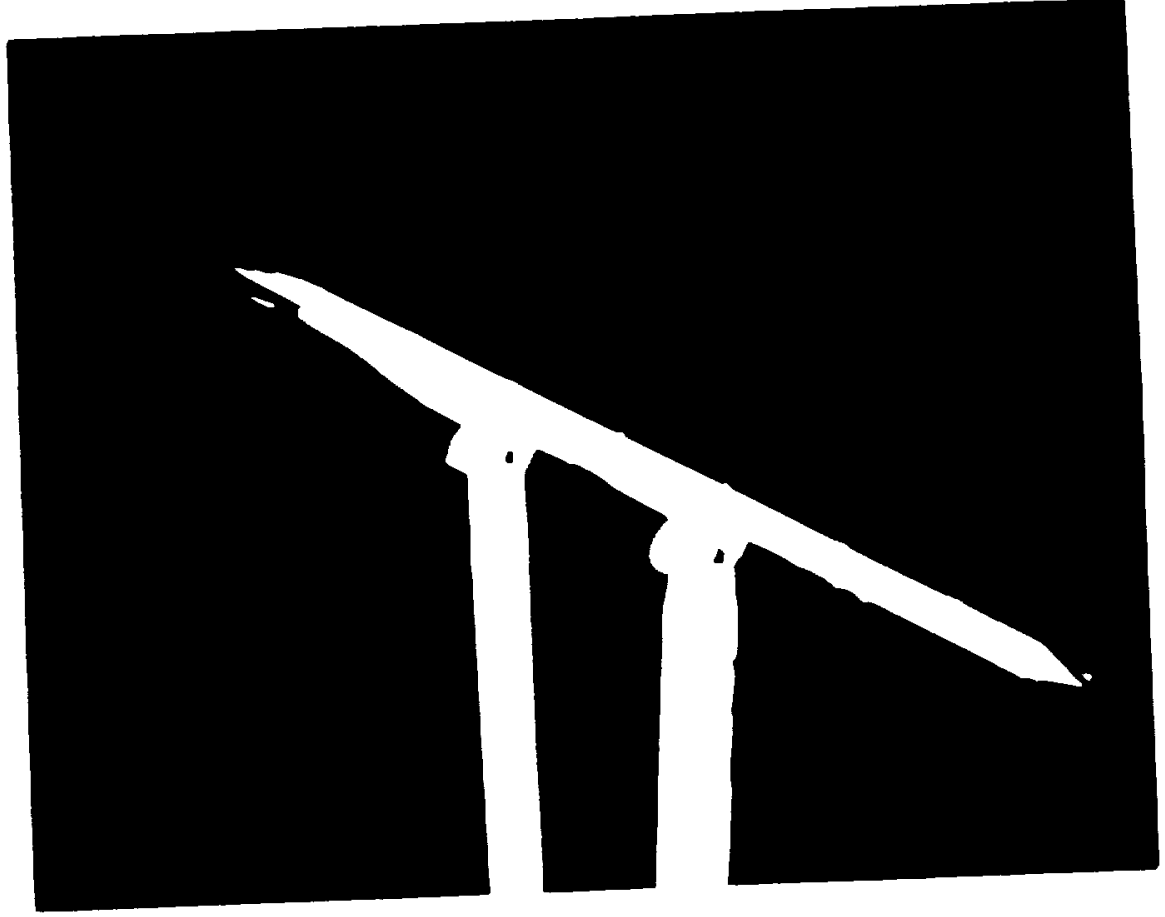

Figure 4.20a: Photograph used to Demonstrate that the Vortex Core can be Assumed to be Straight. Angle of Attack $=29^{\circ}, U=30 \mathrm{ft} / \mathrm{s}$. $\operatorname{Re}=260,000$.

Figure 4.20b: Photograph used to Demonstrate that the Vortex Core can be Assumed to be Straight. Angle of Attack $=39^{\circ}, U=30 \mathrm{ft} / \mathrm{s}$. $\operatorname{Re}=260,000$. 


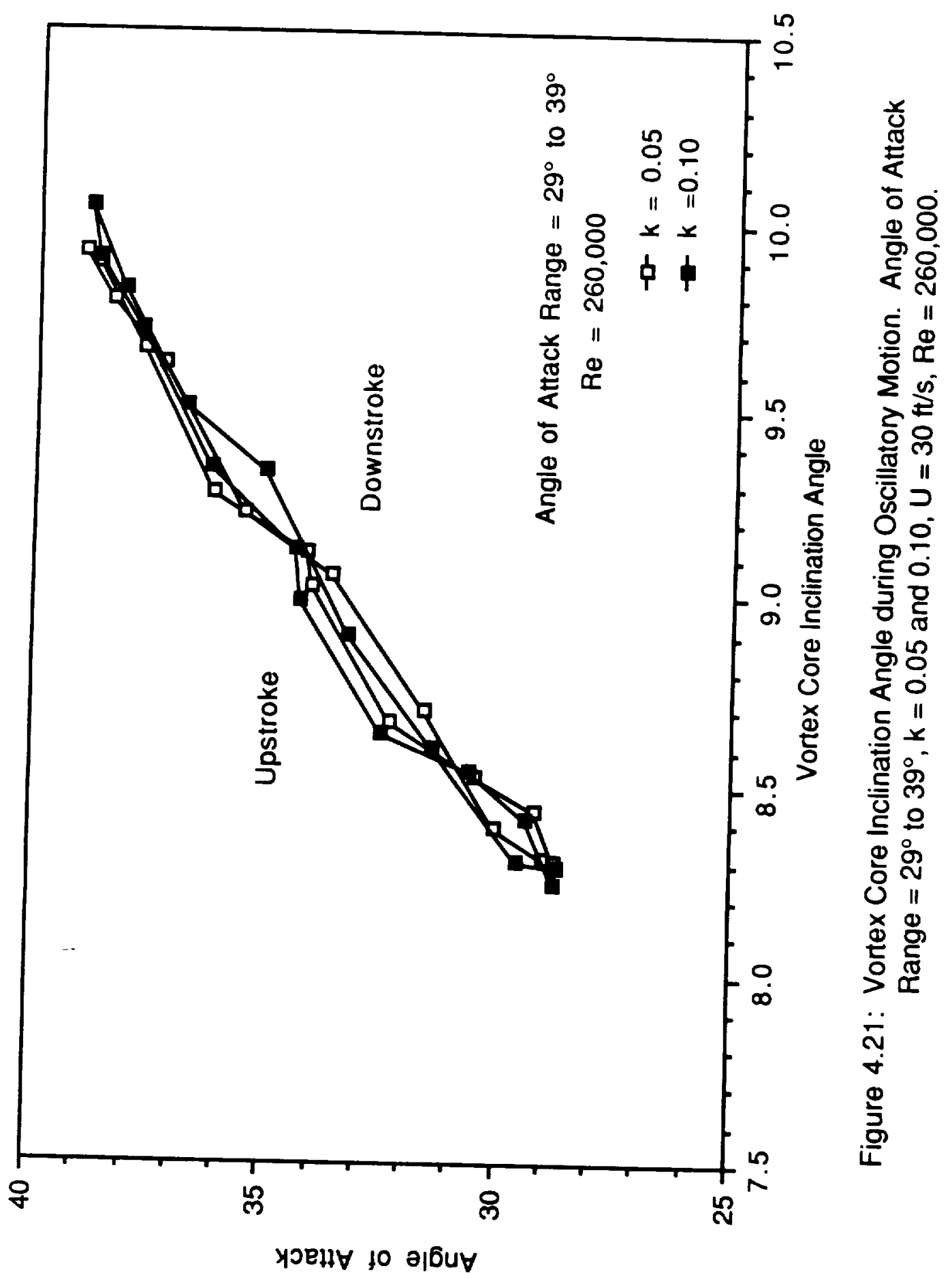




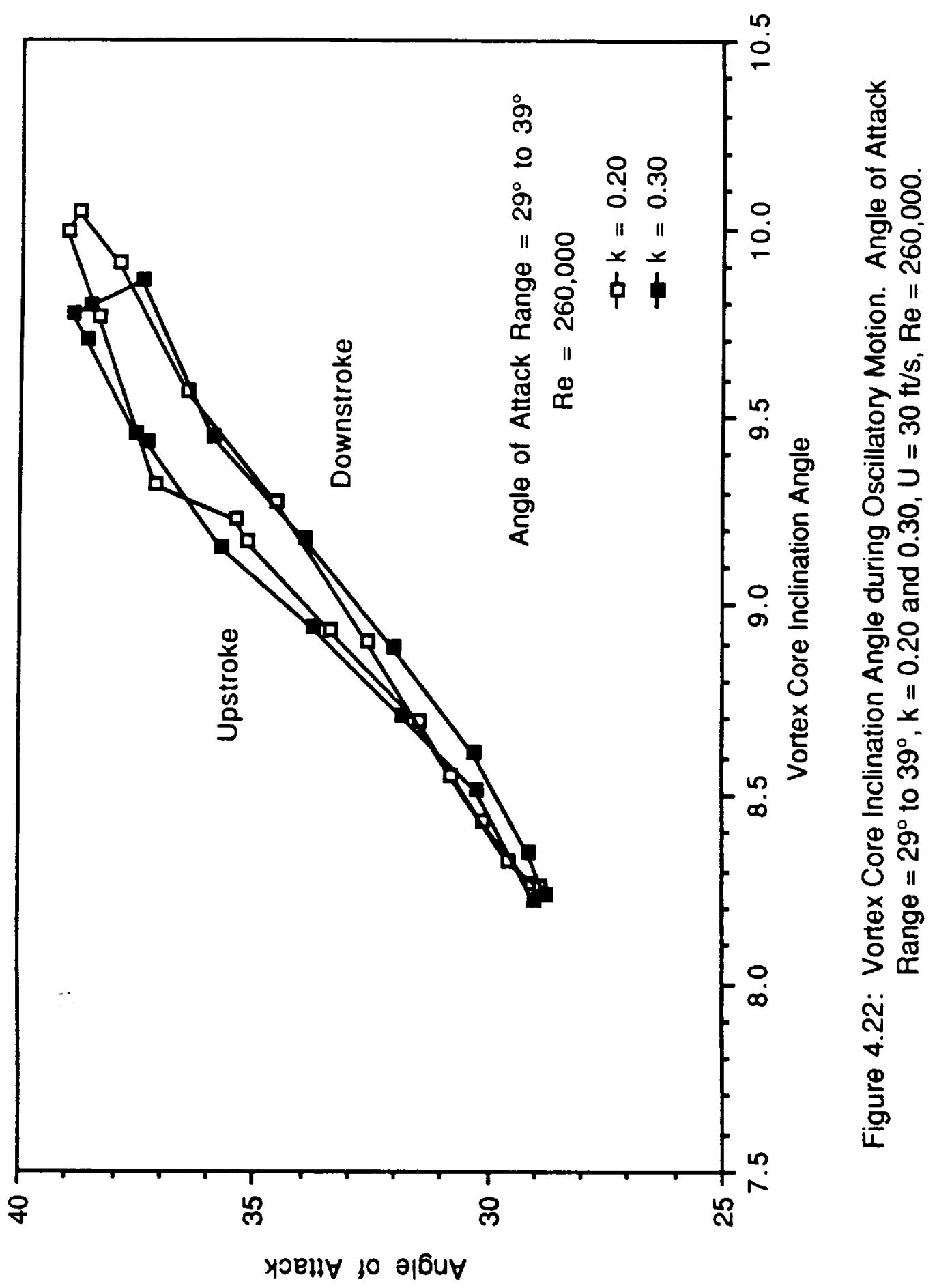




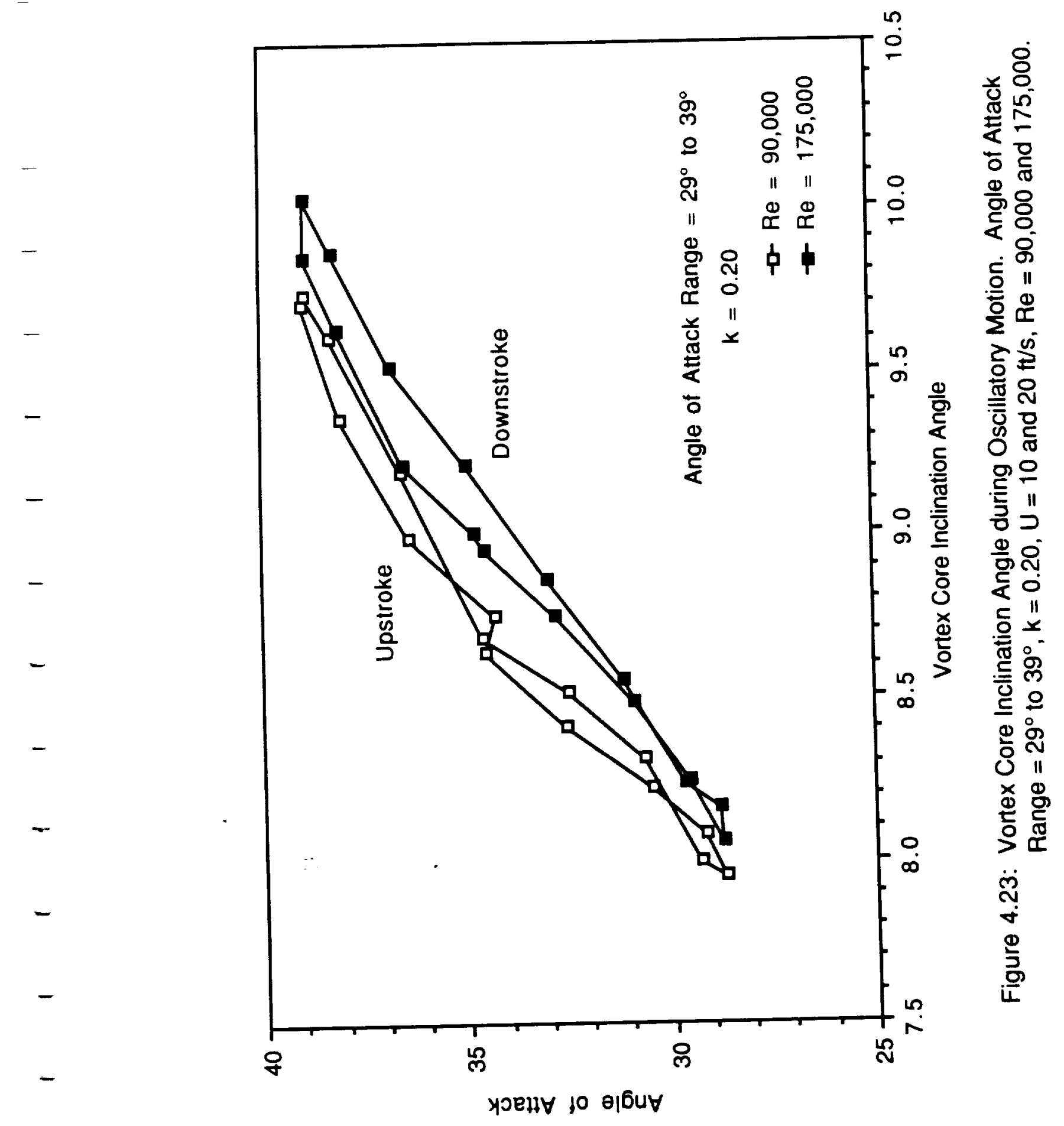




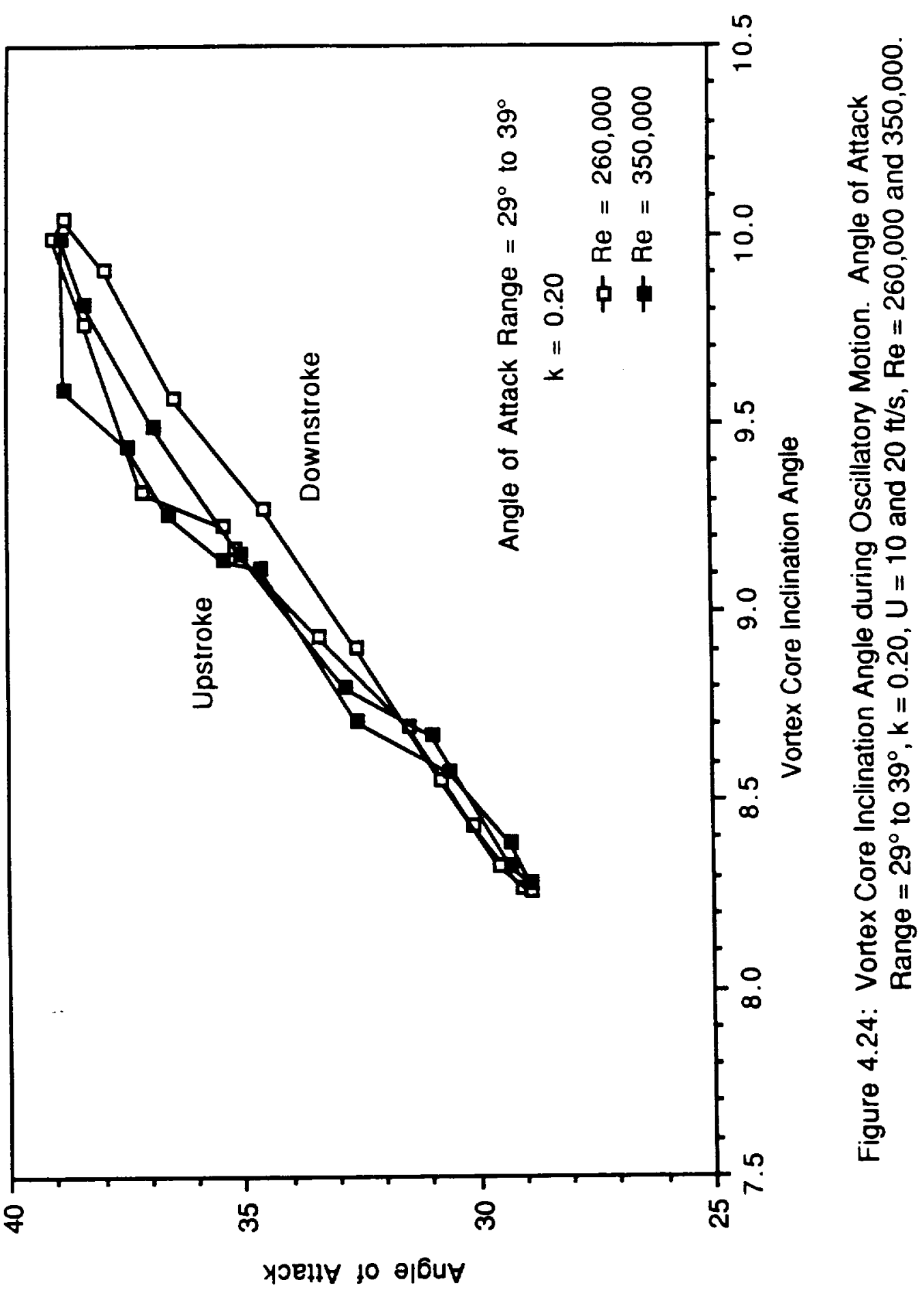



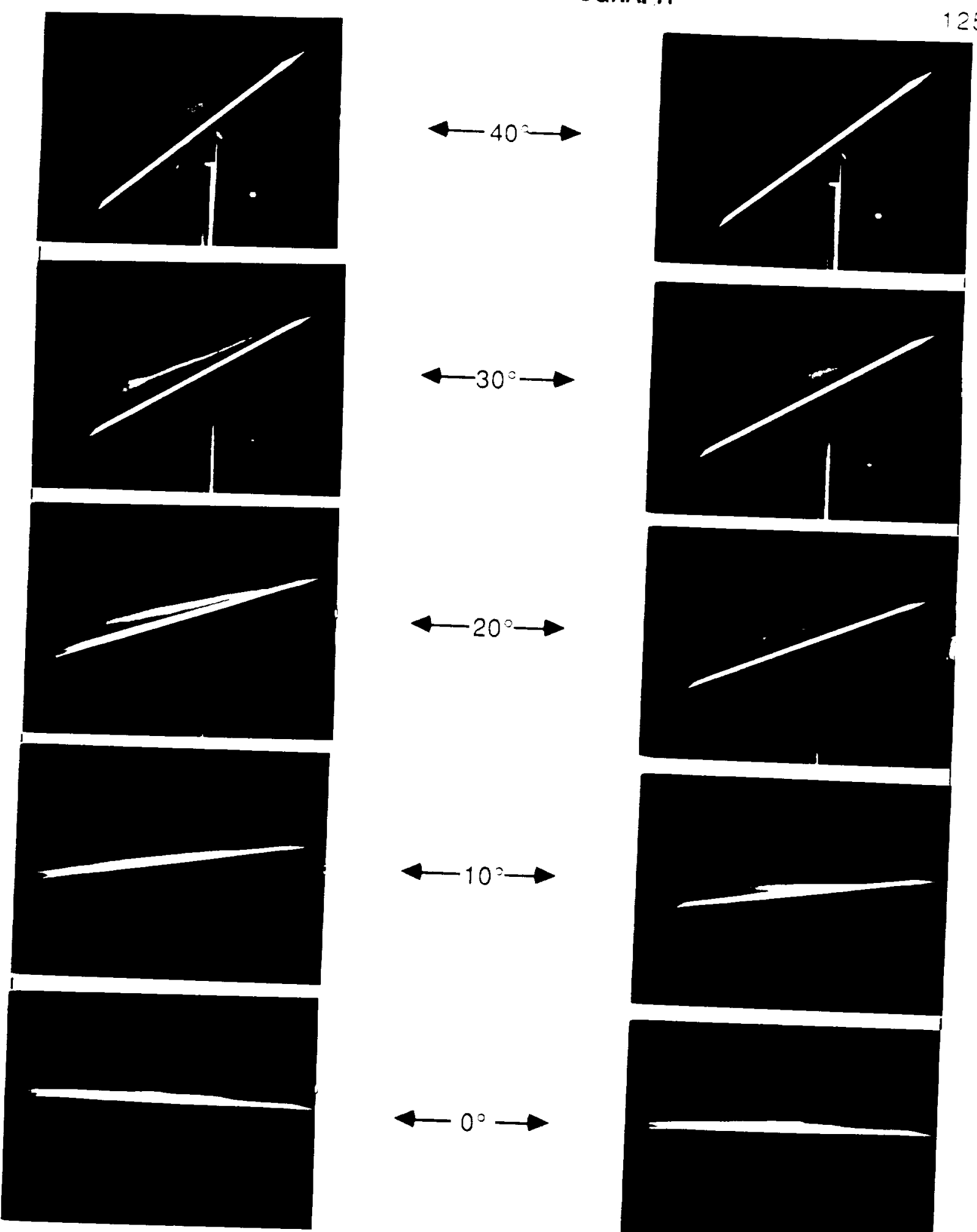

$\alpha$ Increasing

(a-e)
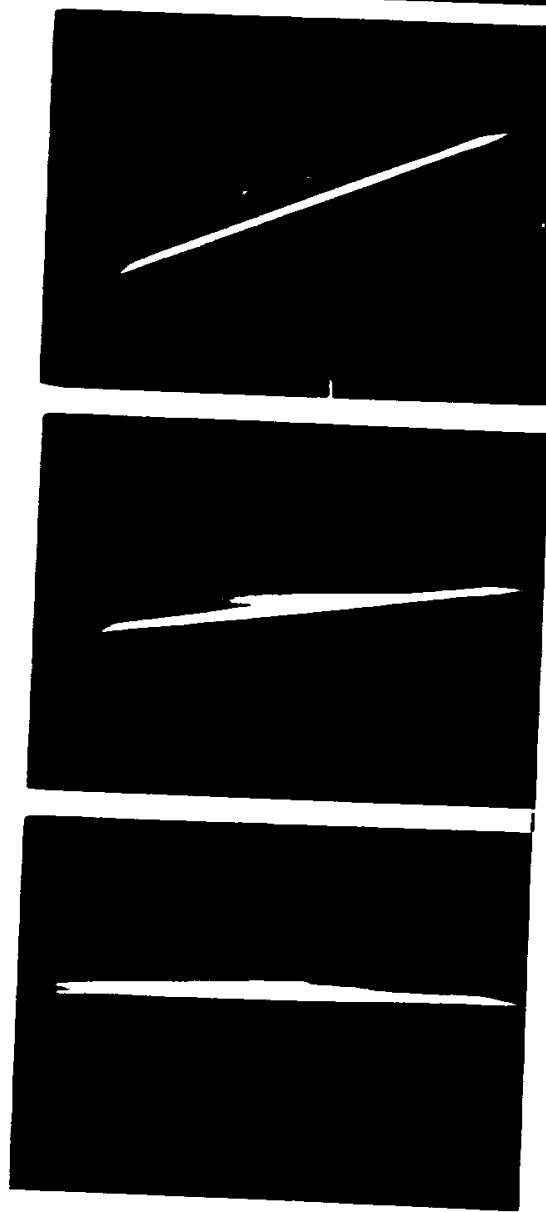

$\alpha$ Decreasing

$(\mathfrak{f} \cdot \mathrm{j})$

Figure 4.25a-j: Sequence of Photographs made from $16 \mathrm{~mm}$ Movie Film which Illustrates how the Chordwise Breakdown Location varies throughout a Pitching Cycle. Angle of Attack Range $=00^{\circ}$ to $45^{\circ}$. $k=0.30, U=30 \mathrm{tt} / \mathrm{s}, \operatorname{Re}=260,000$. 

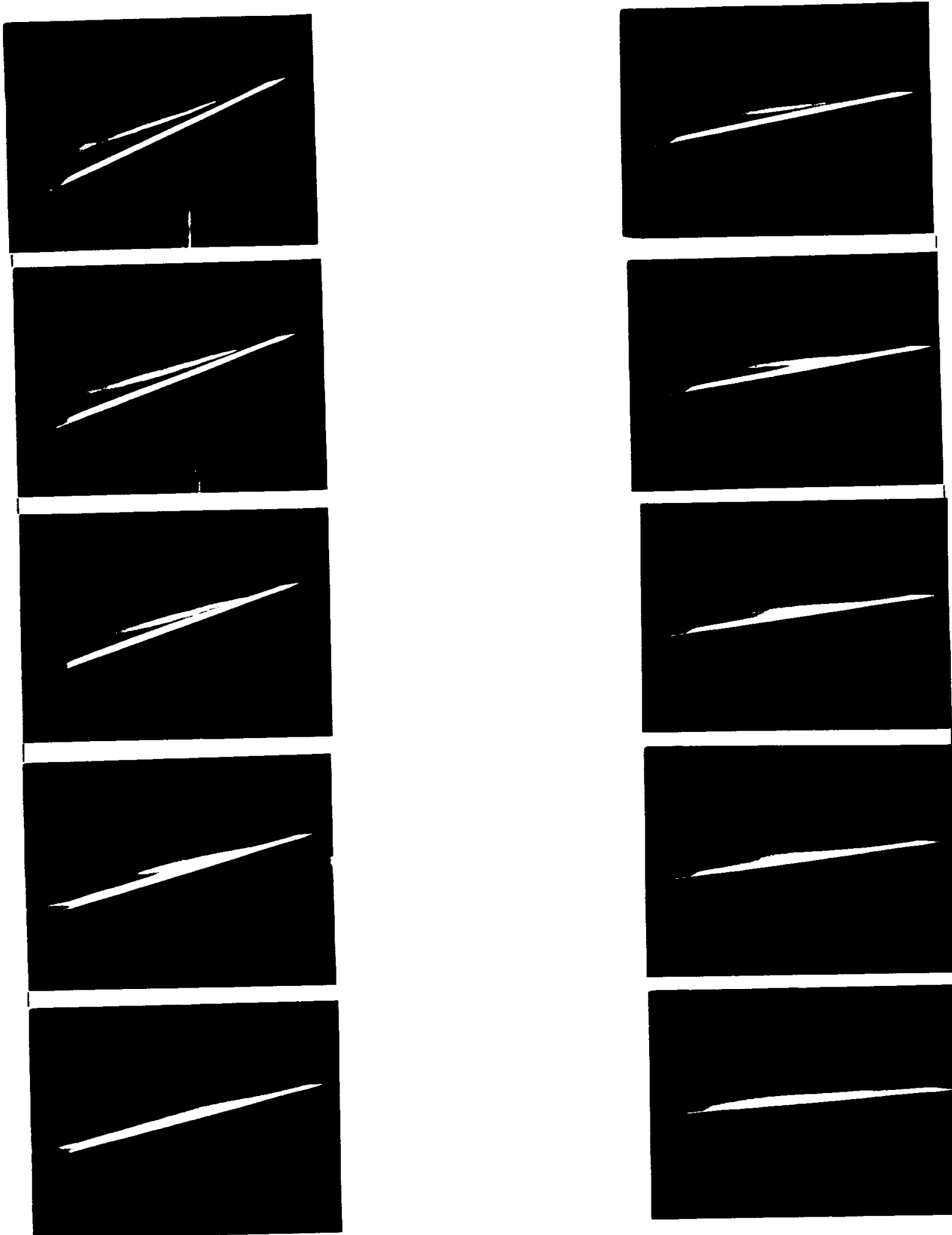

Separation

$(\mathrm{a}-\mathrm{e})$

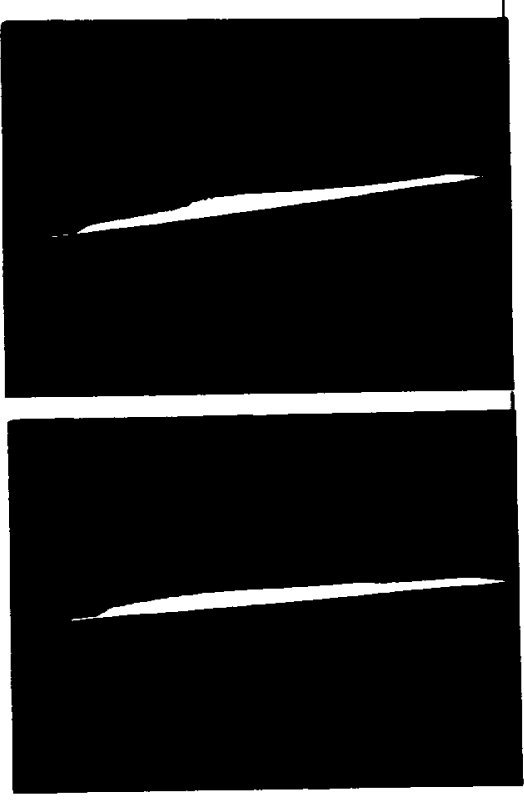

Reattachment

$(f-j)$

Figure 4.26a-j: Sequence of Photographs made from $16 \mathrm{~mm}$ Movie Film which Illustrates the Separation and Reattachment Processes of the Vortex Flow. 


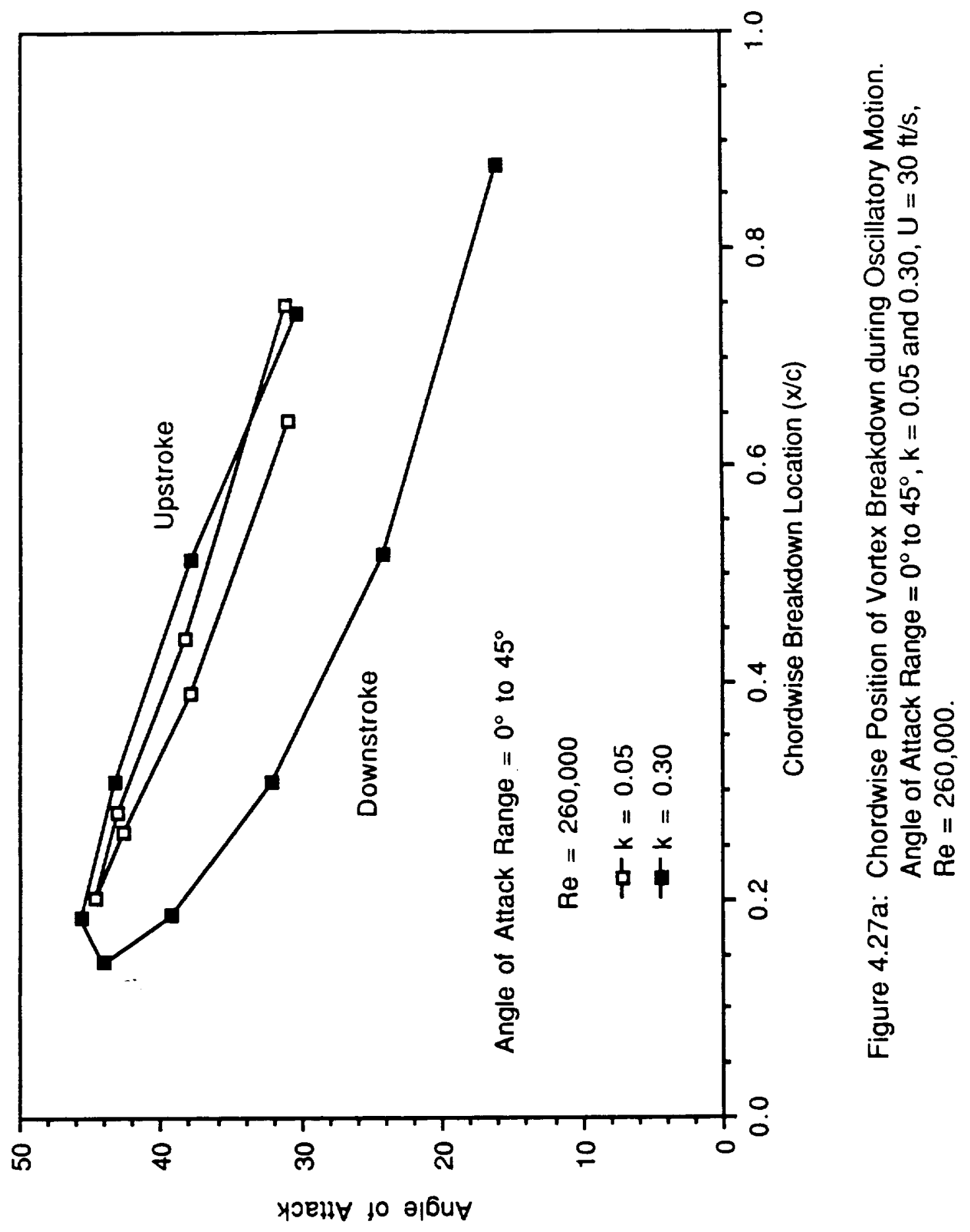




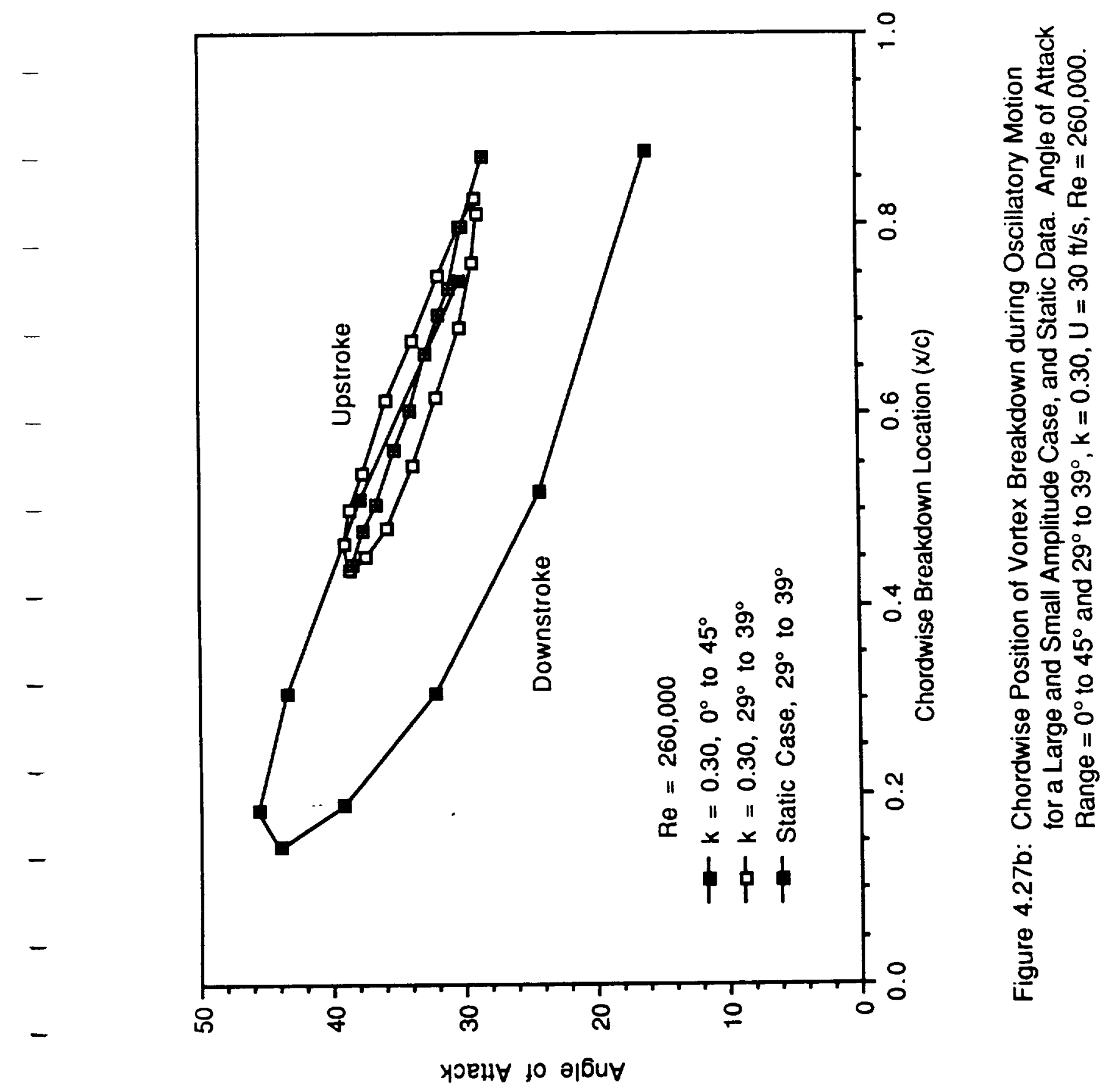




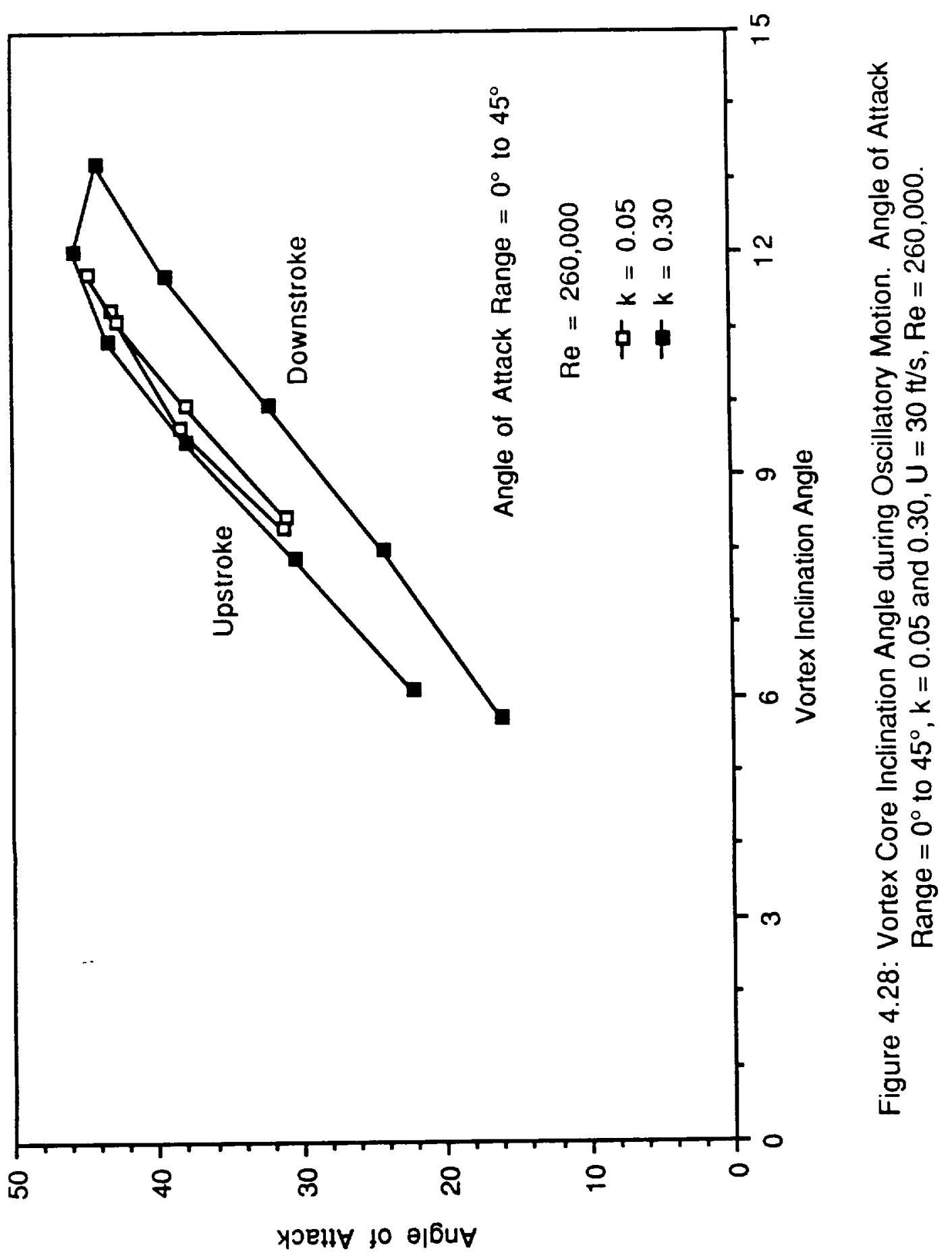


(o/x) Uо!ाеsoา uморуеадg әs!mpjouว

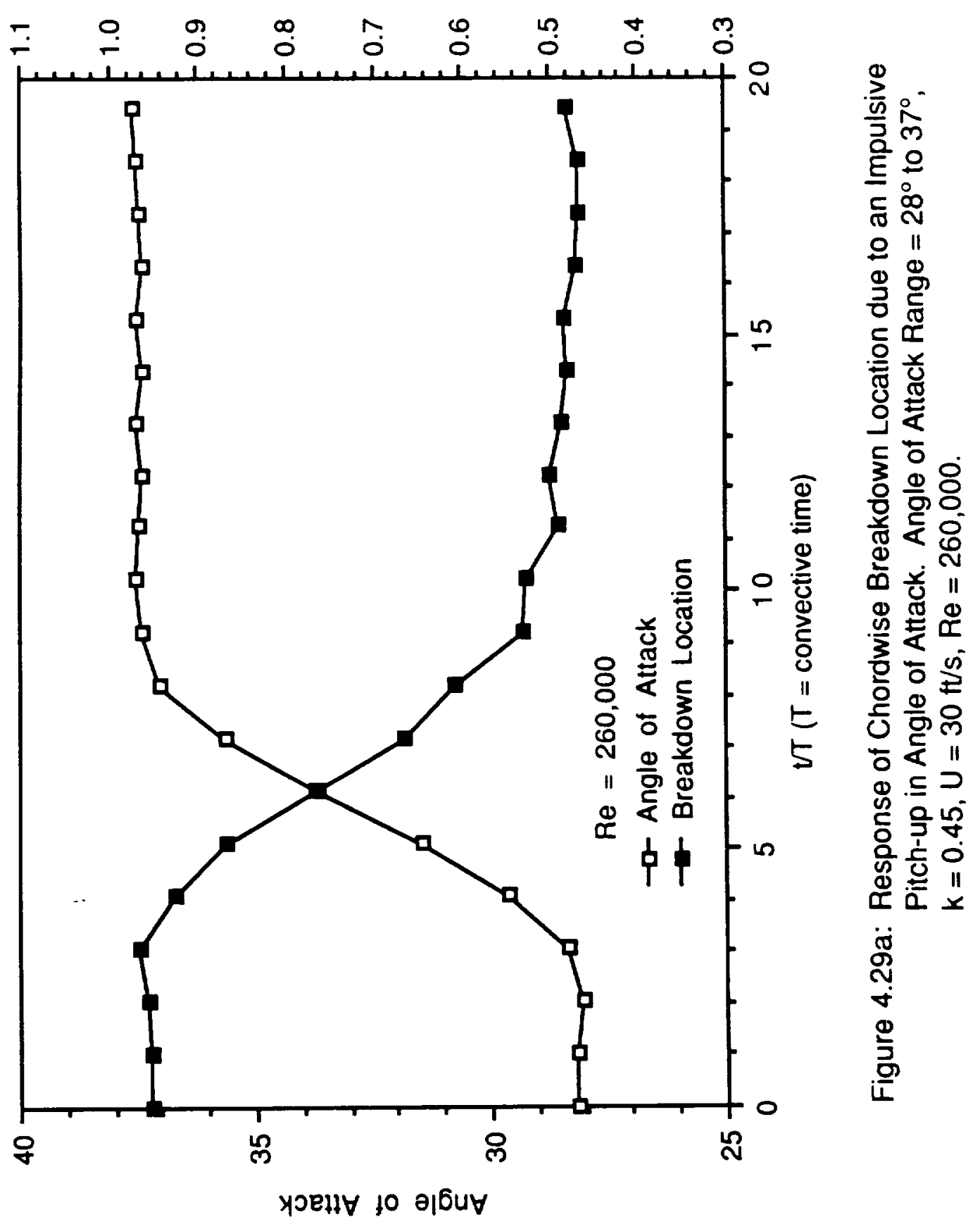




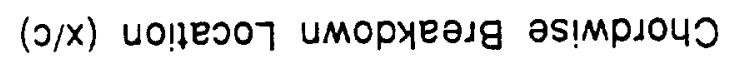

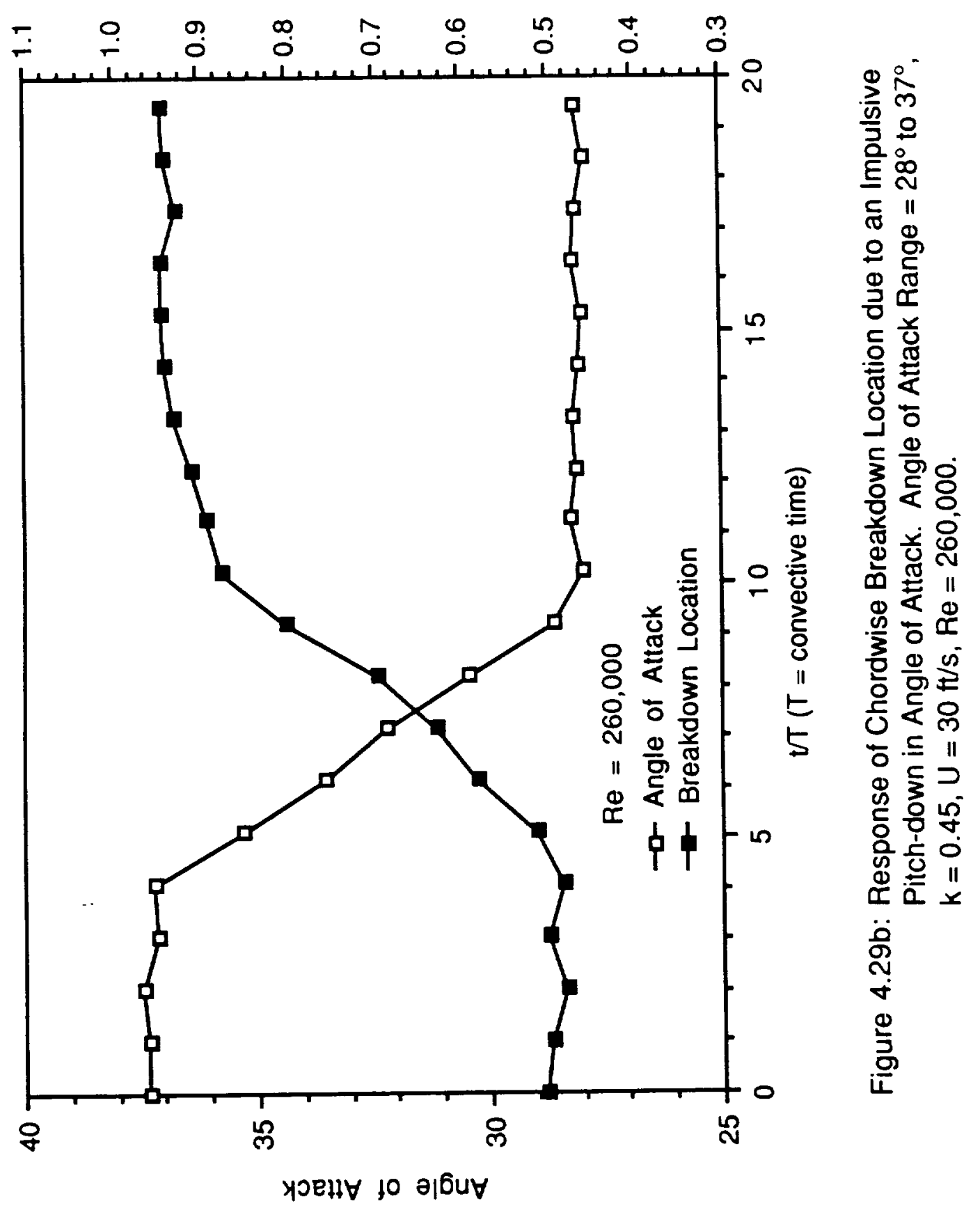




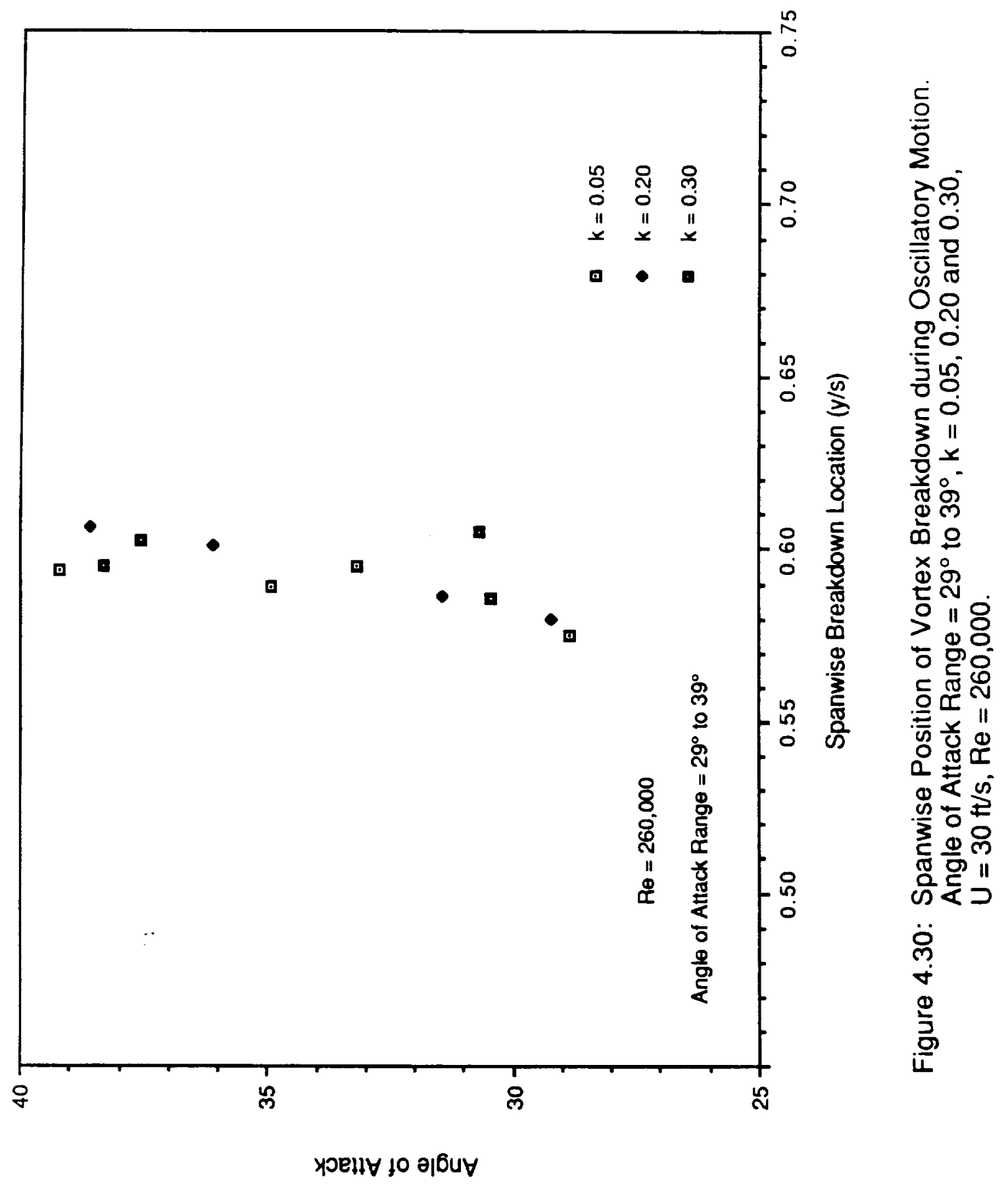




\section{APPENDIX A}

Discrete Fourier Transforms of Model Motion 


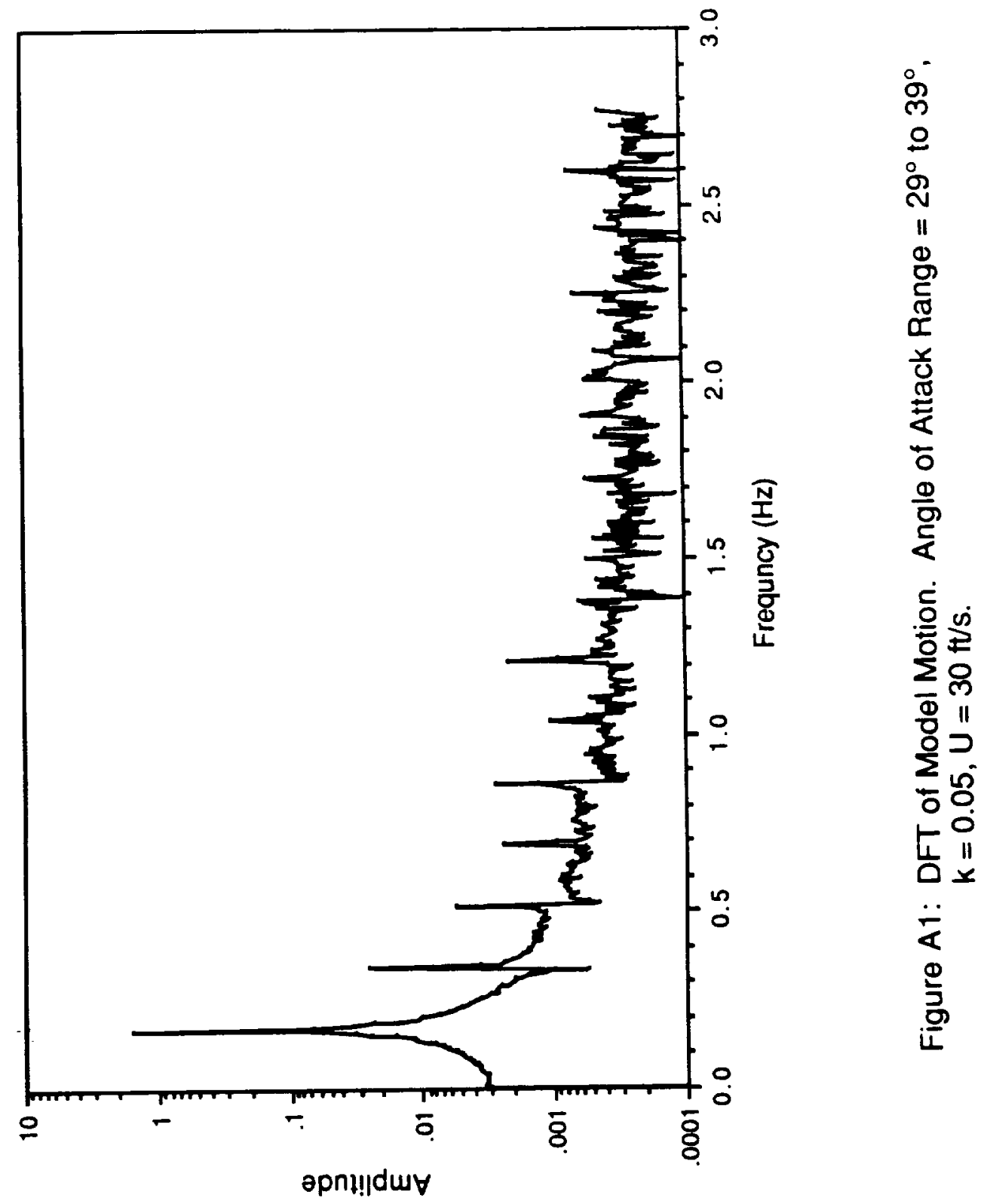




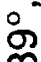

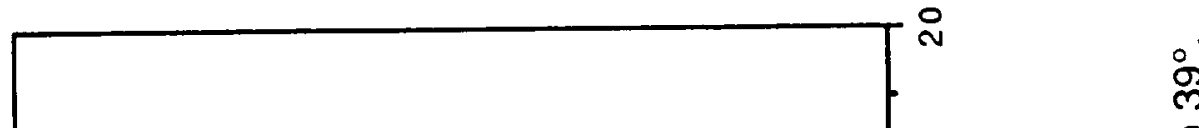

으

오

(

әpn!!|dwళ

号

准

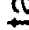

遅

음

䢘

둥

음로

$\sum$ 으

ब

כ

$\sum 0$

늠음

غั

ํํำ

it 


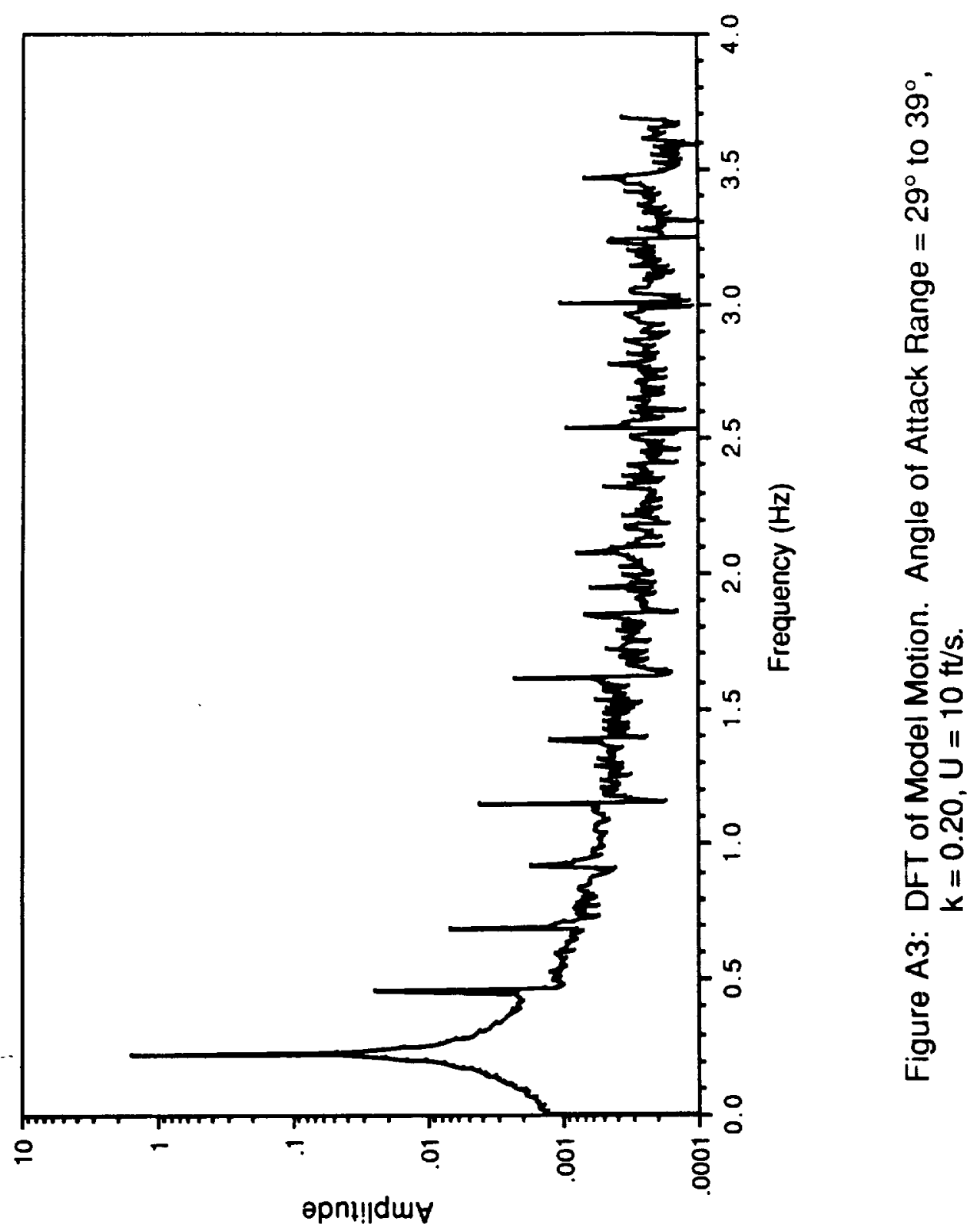




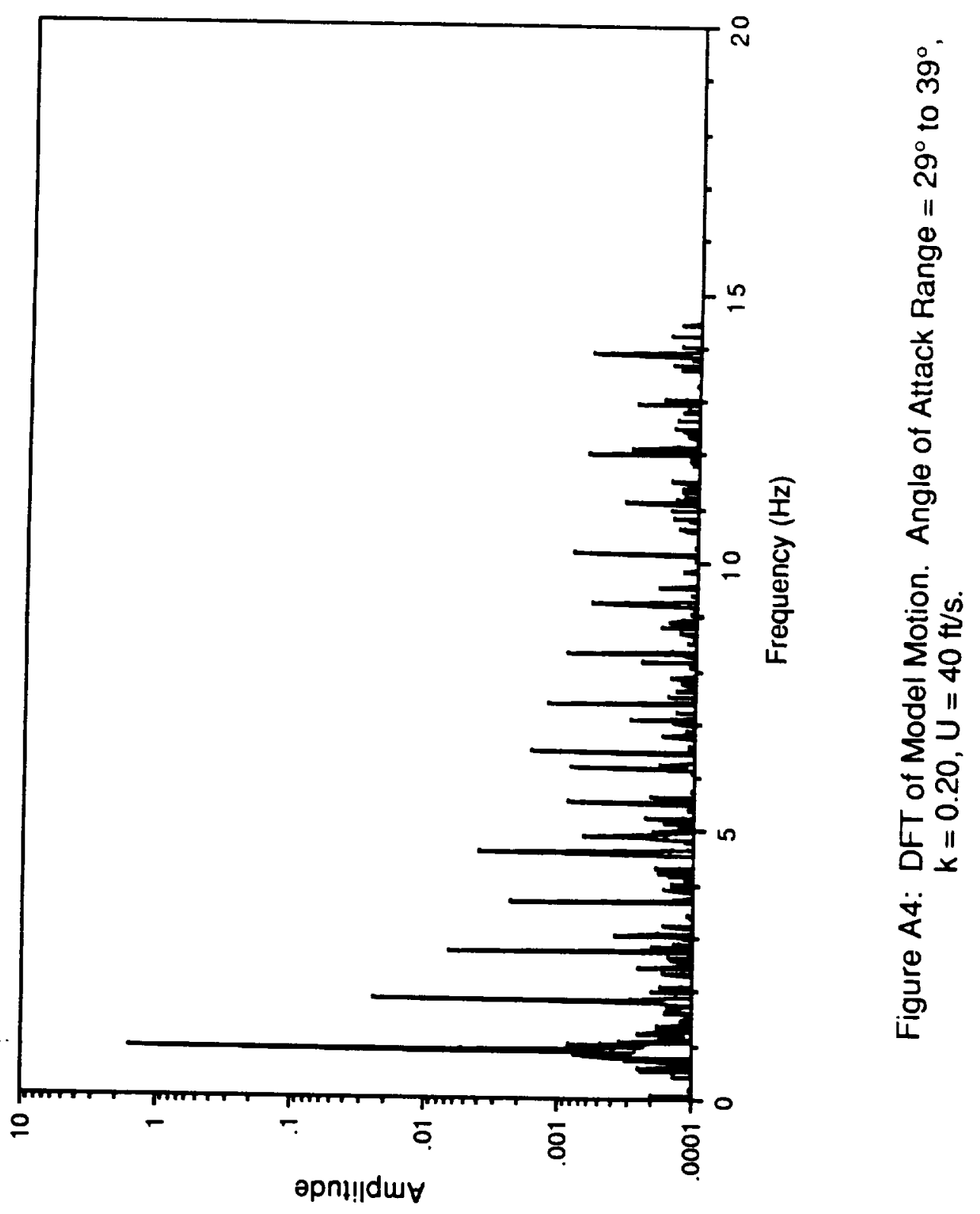




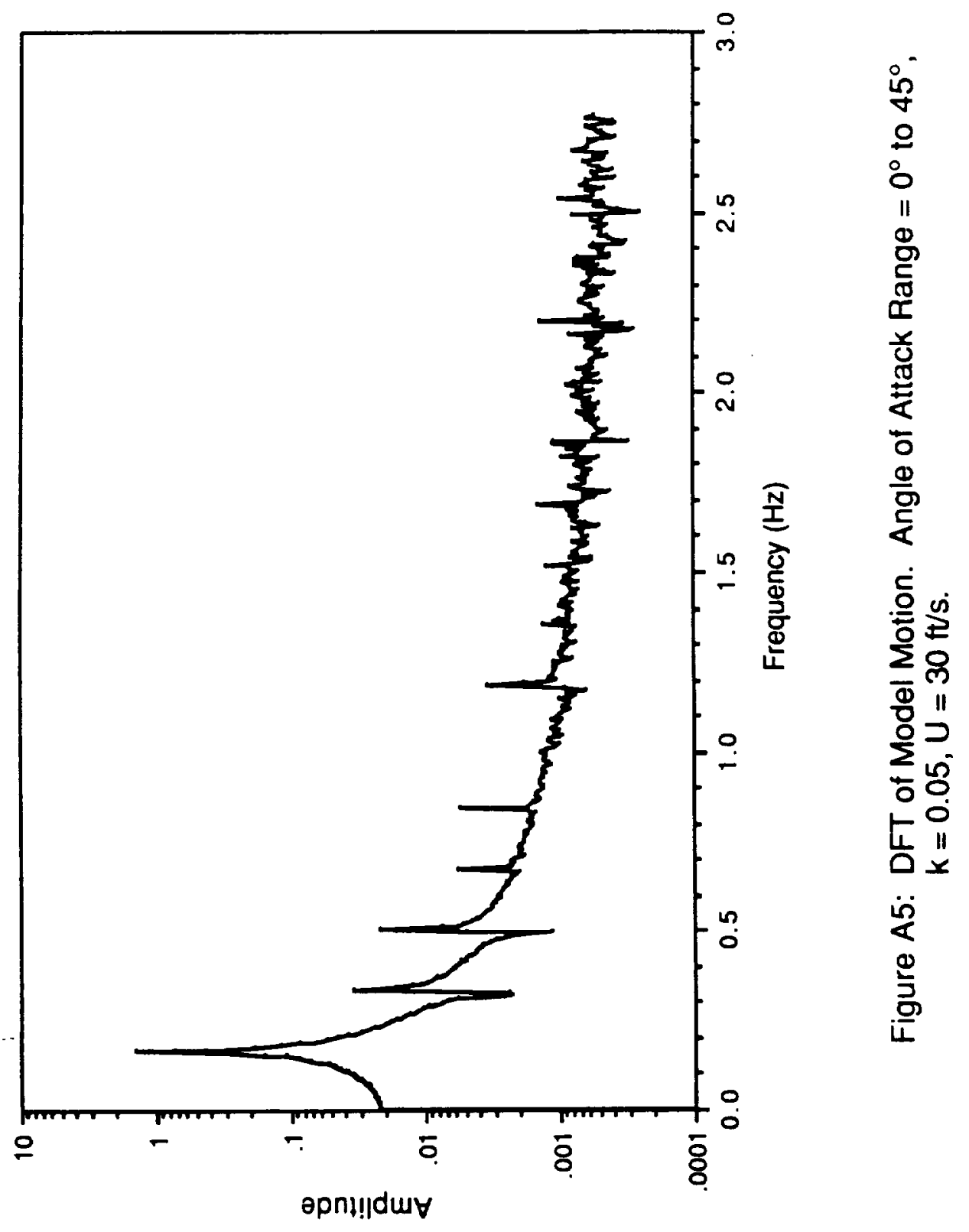




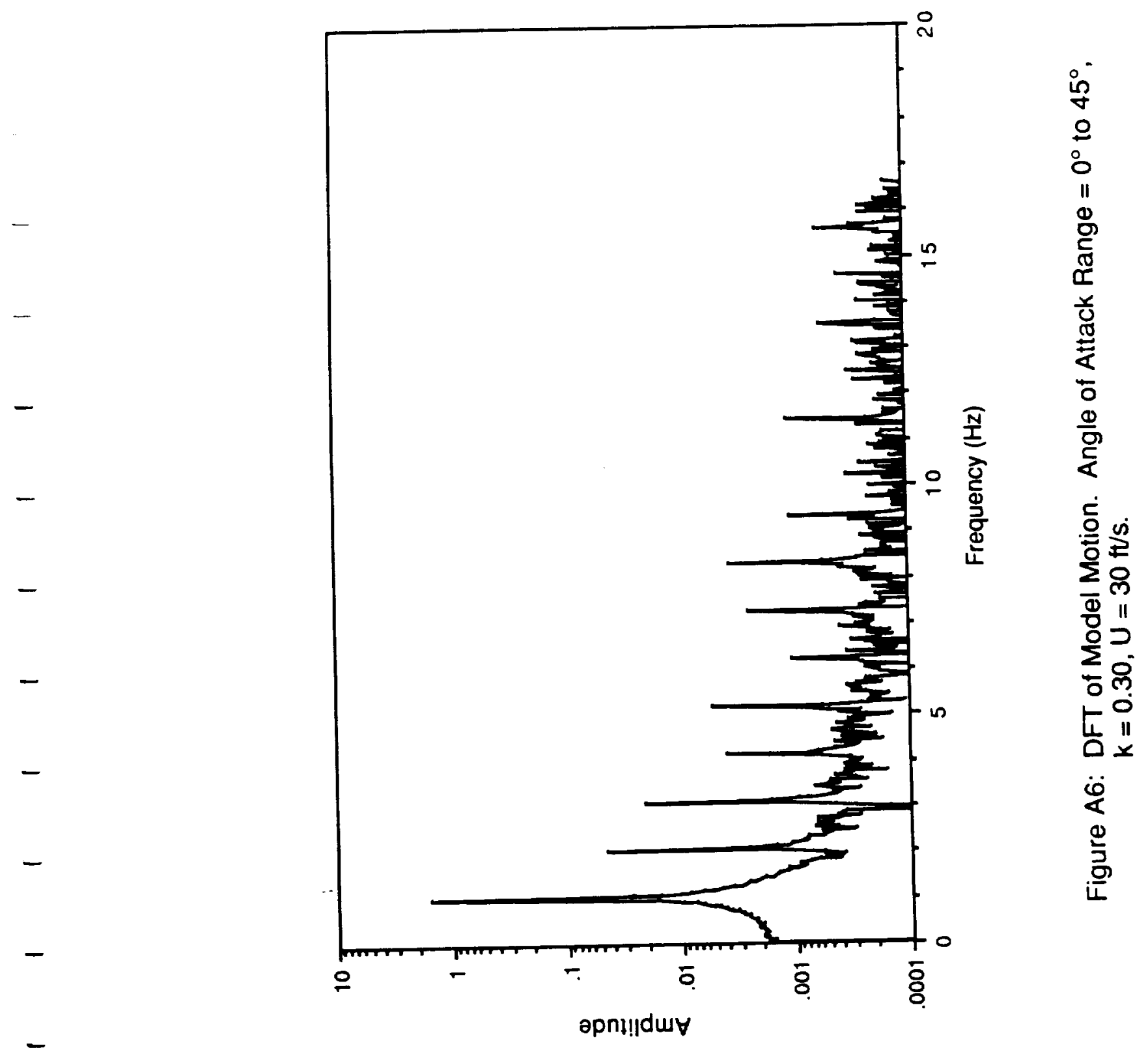




\section{APPENDIX B}

Freestream Velocity Fluctuation and Model Position 


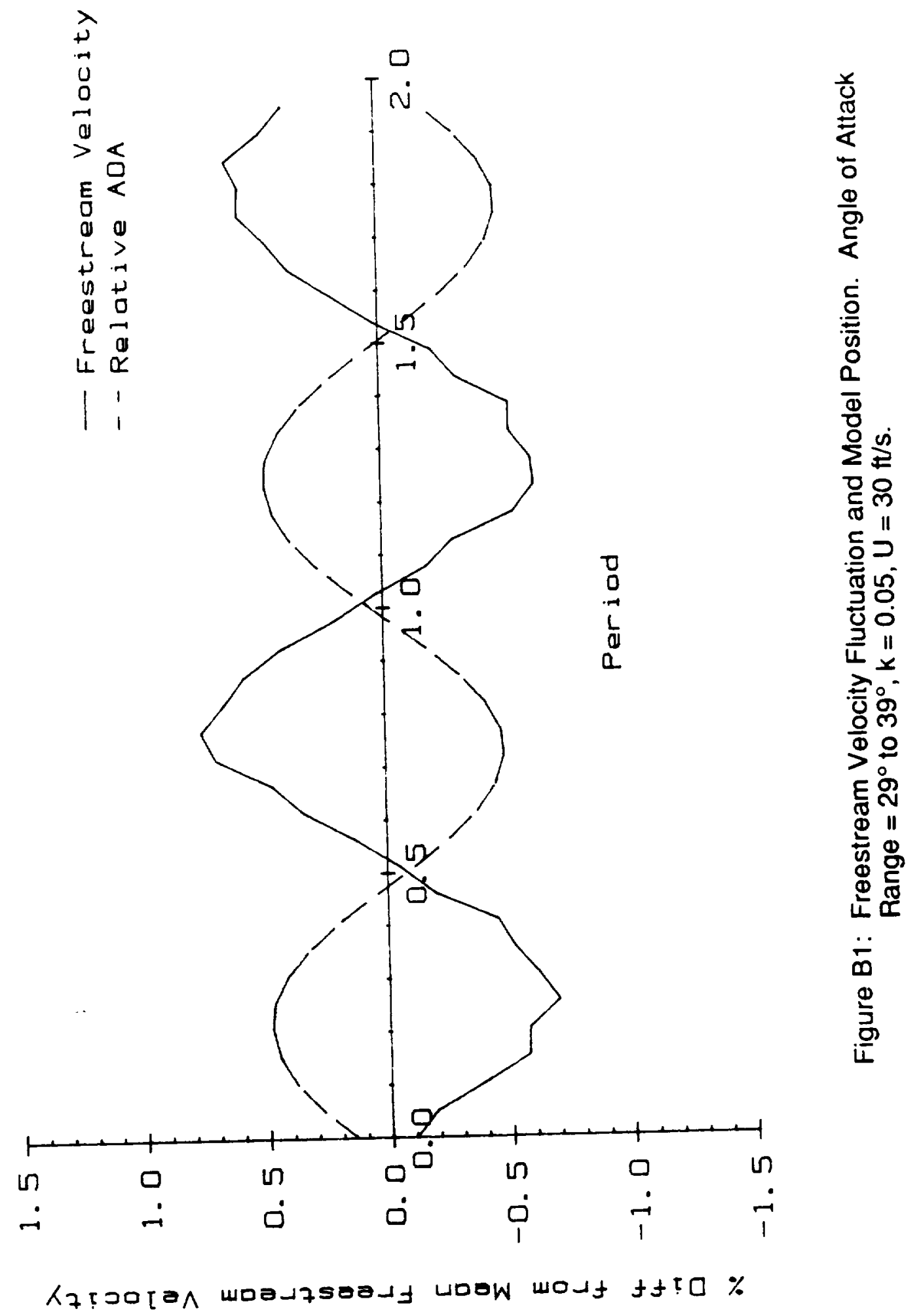




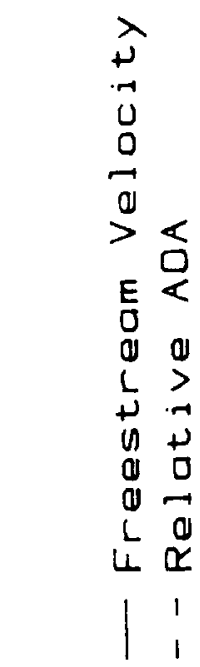

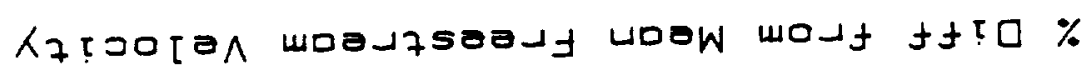




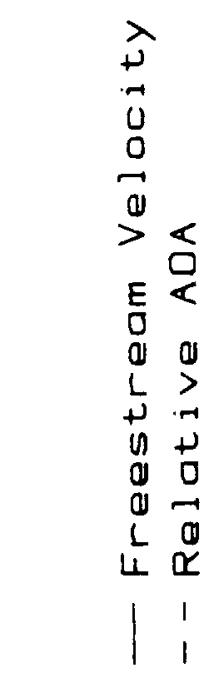

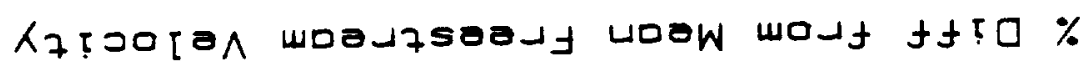


$\widehat{+}$
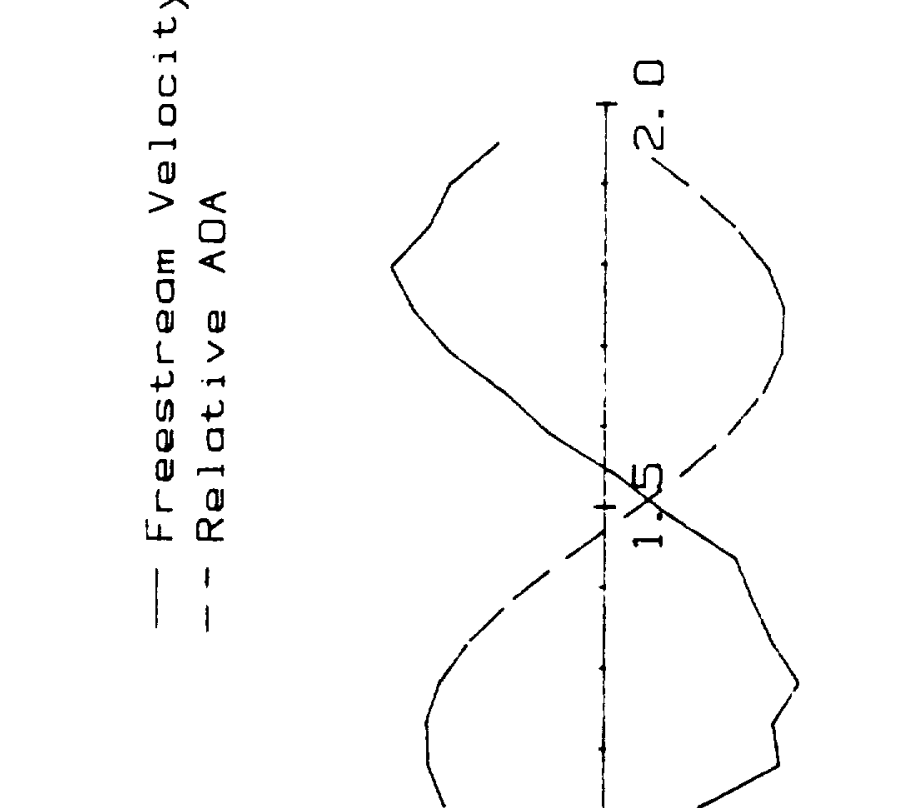

$\underset{\alpha}{\alpha}$

0
0
$\frac{1}{0}$
0

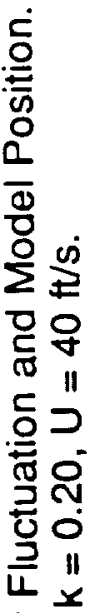

‥

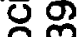

응

ह 용

๑ี II

फ

Ð

比嵒

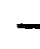

$-$

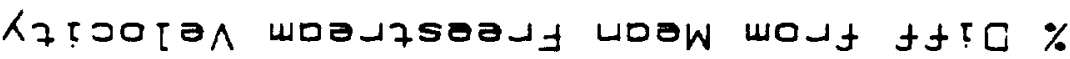




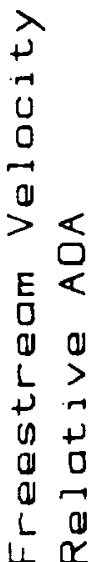

$1 \alpha$

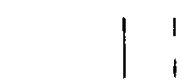

$-$

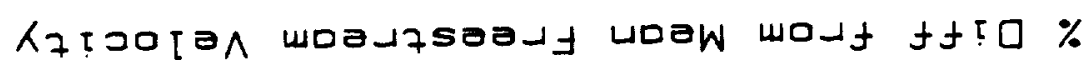




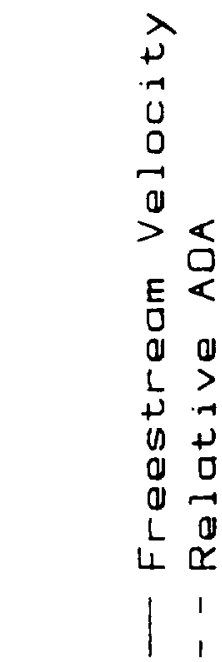

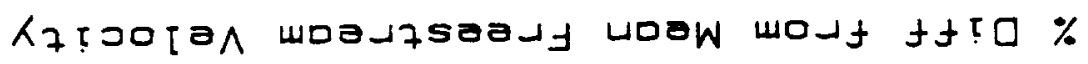




\section{APPENDIX C}

Chordwise Breakdown Location and Model Position 


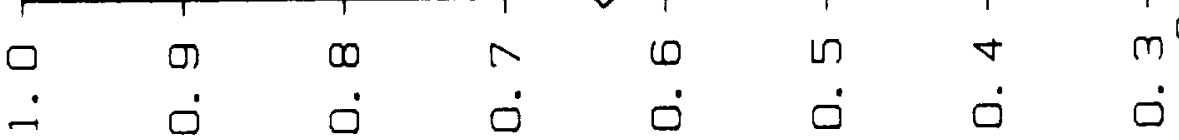

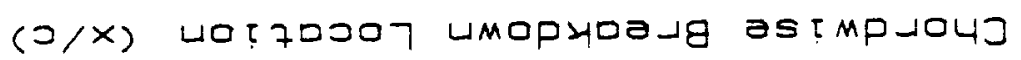




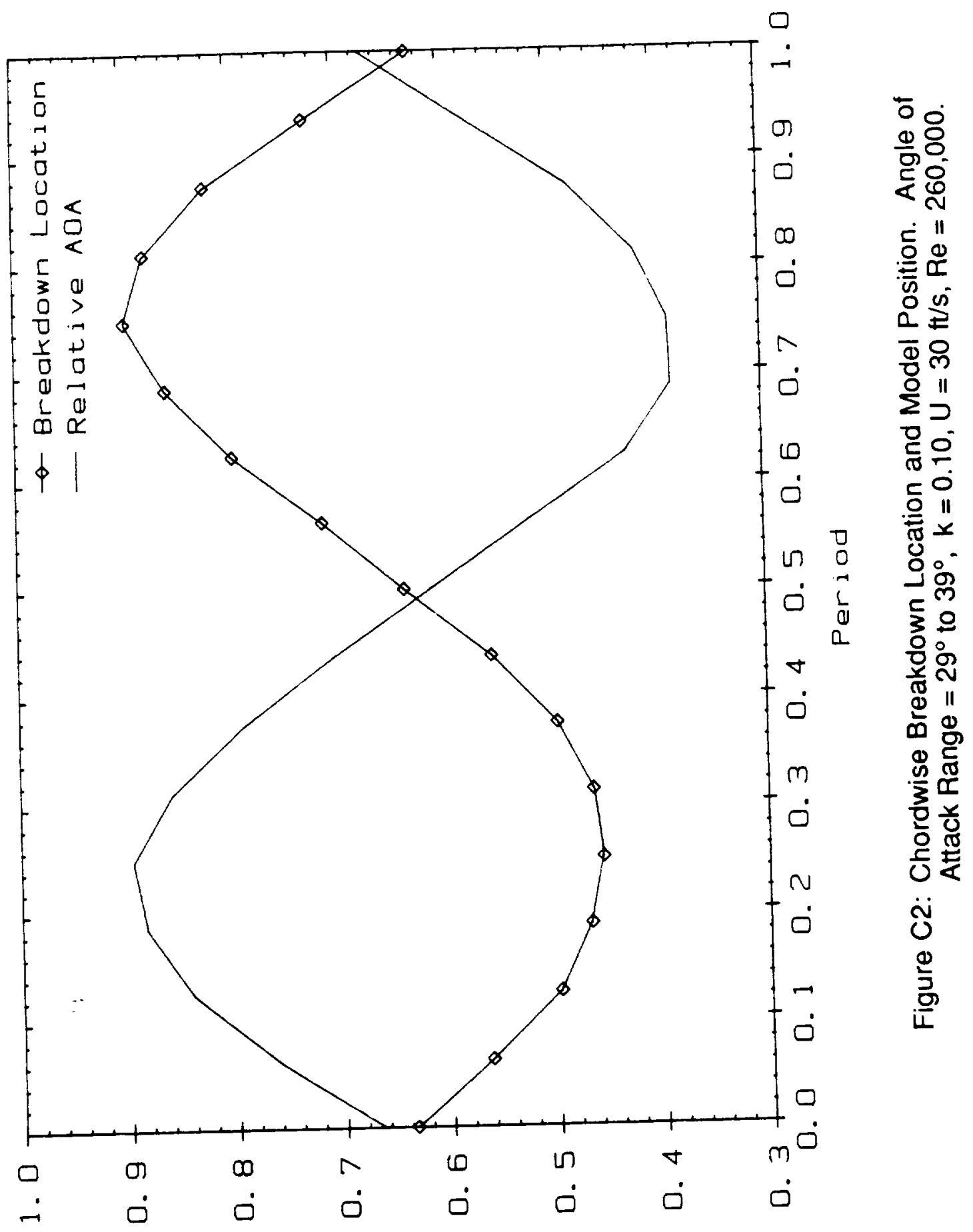

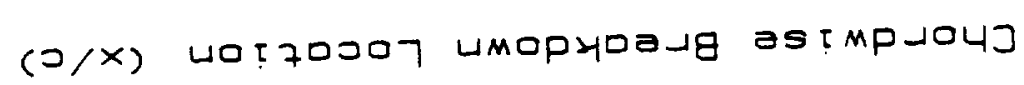




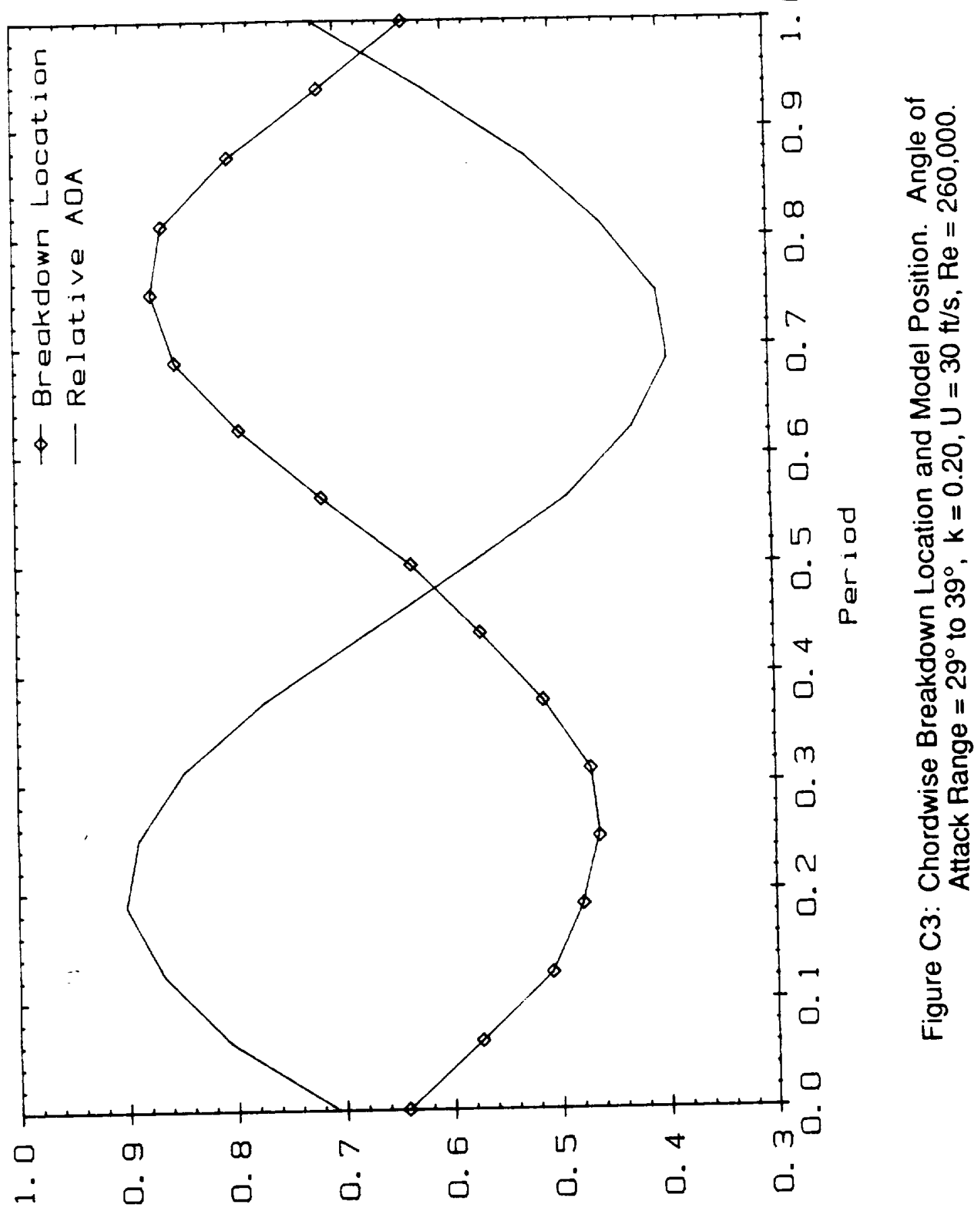

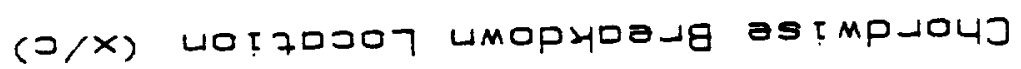




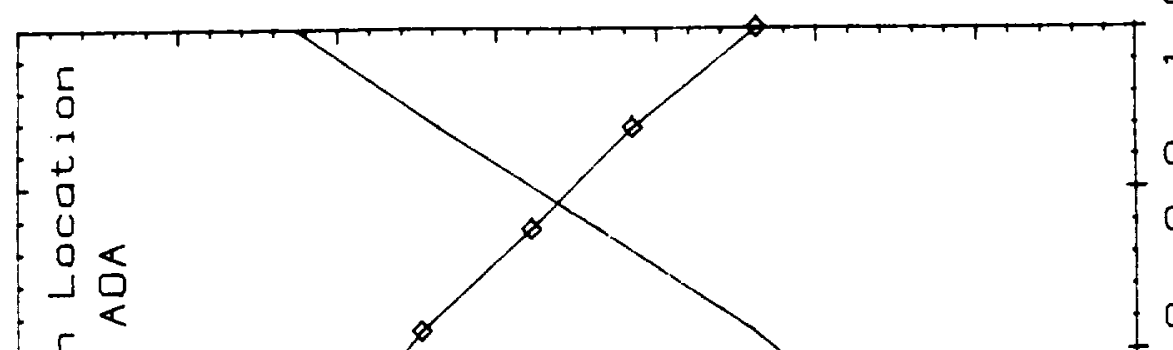

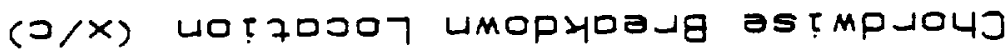


ธ

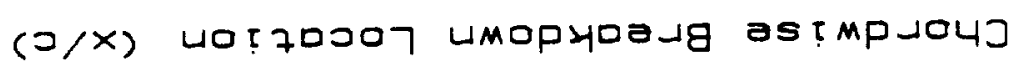




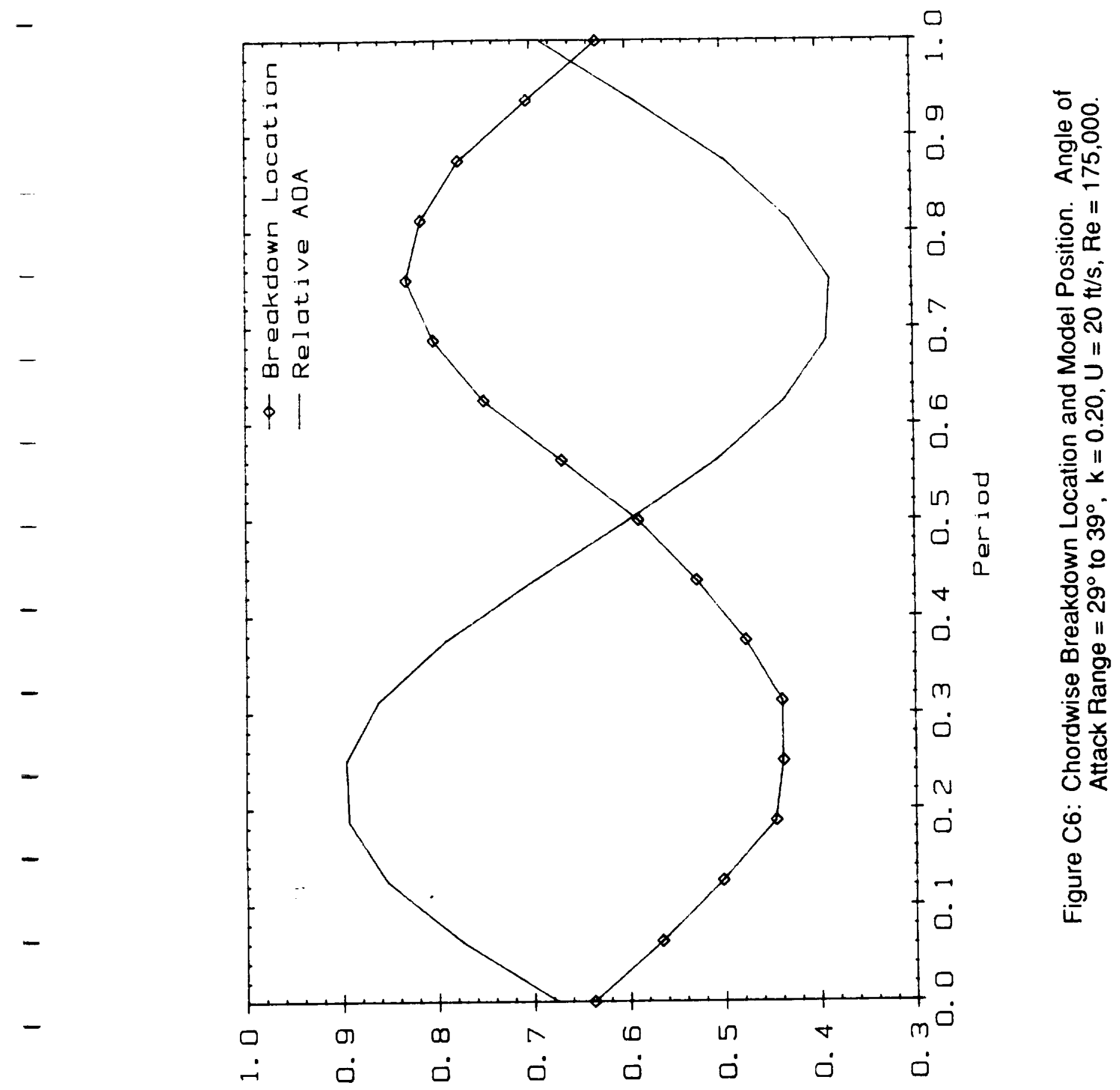

$$
\text { (כ/x ( }
$$




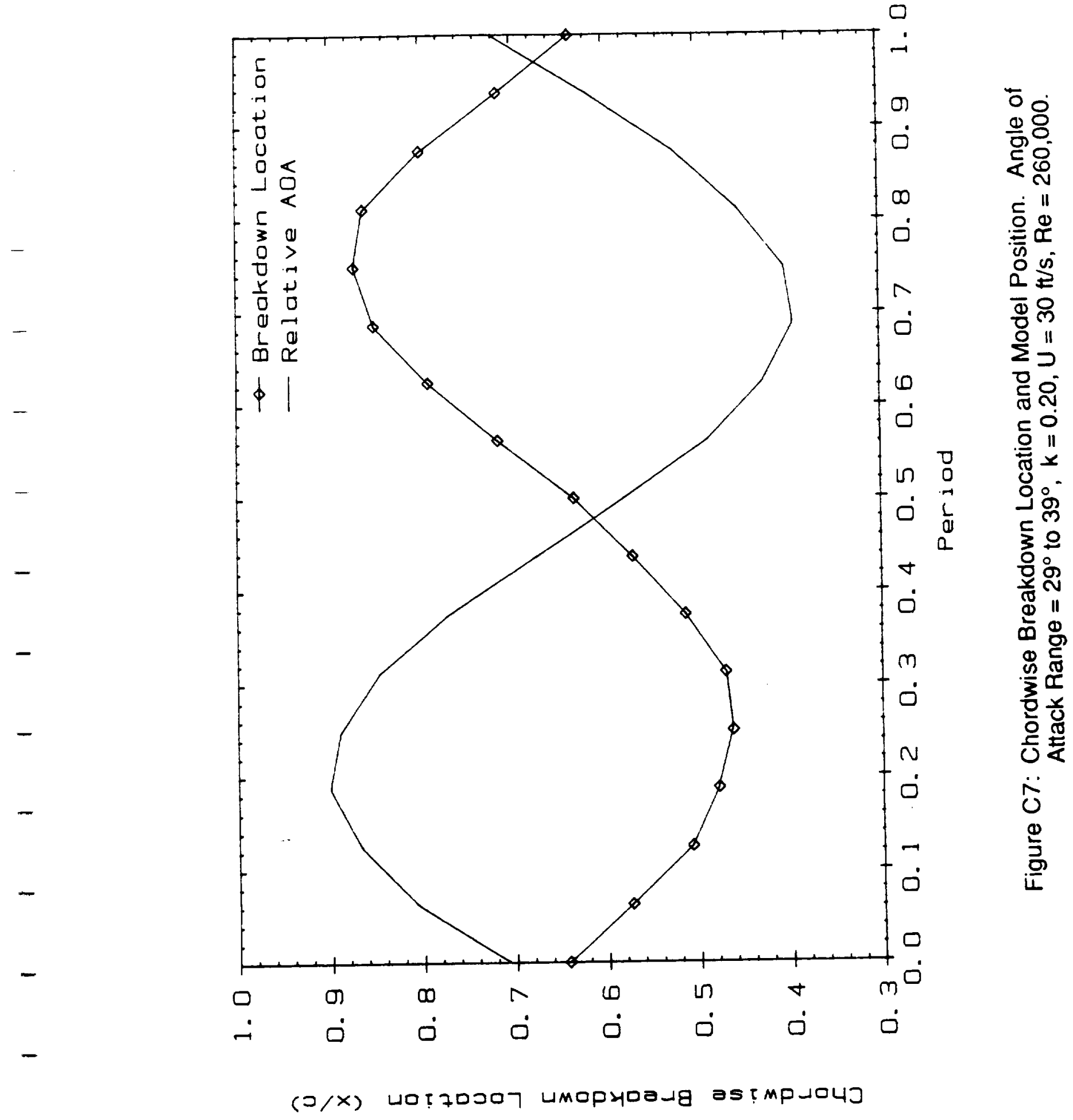




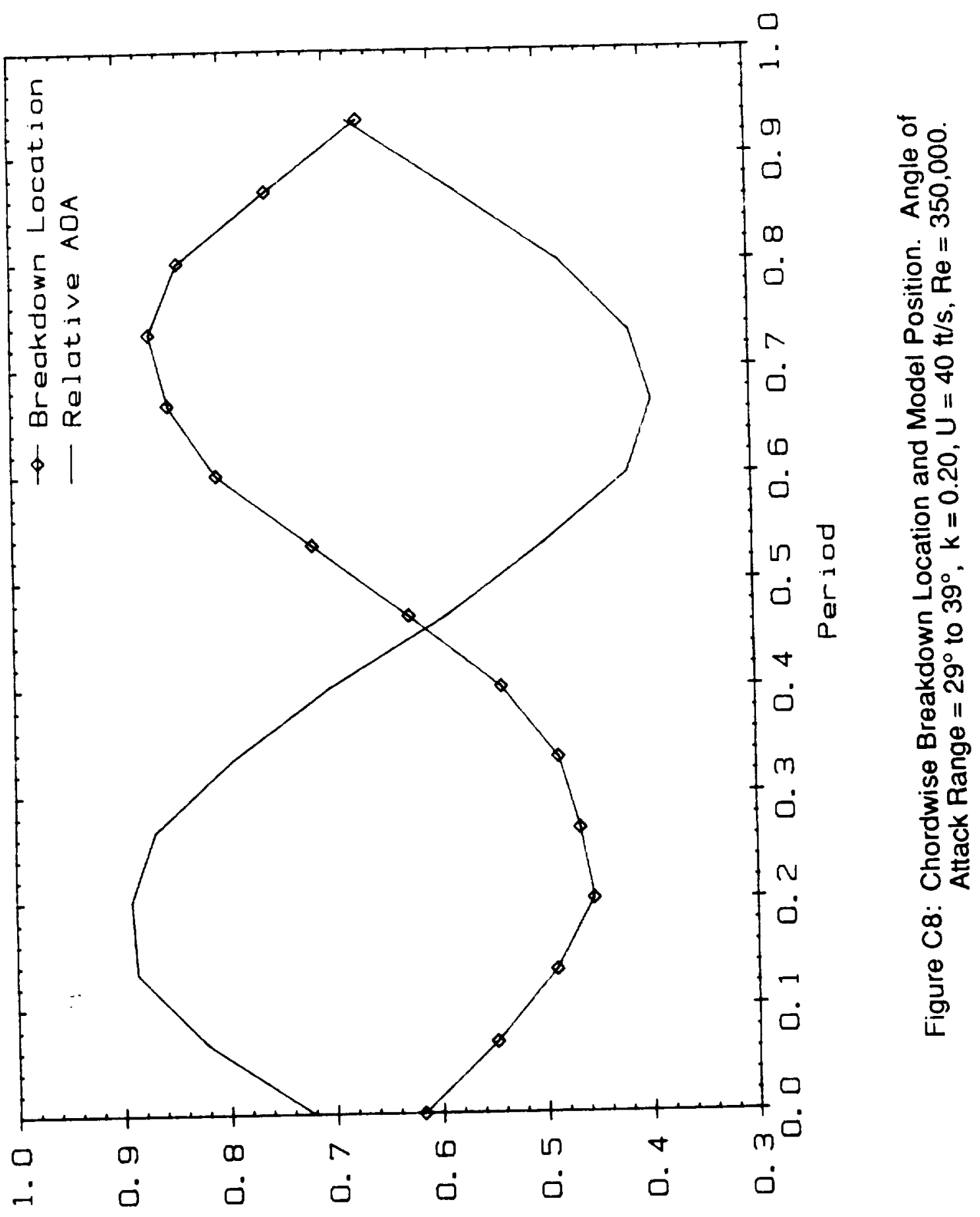

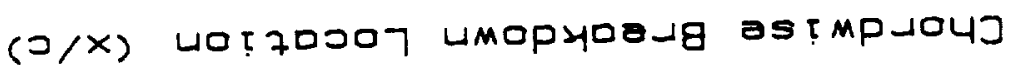




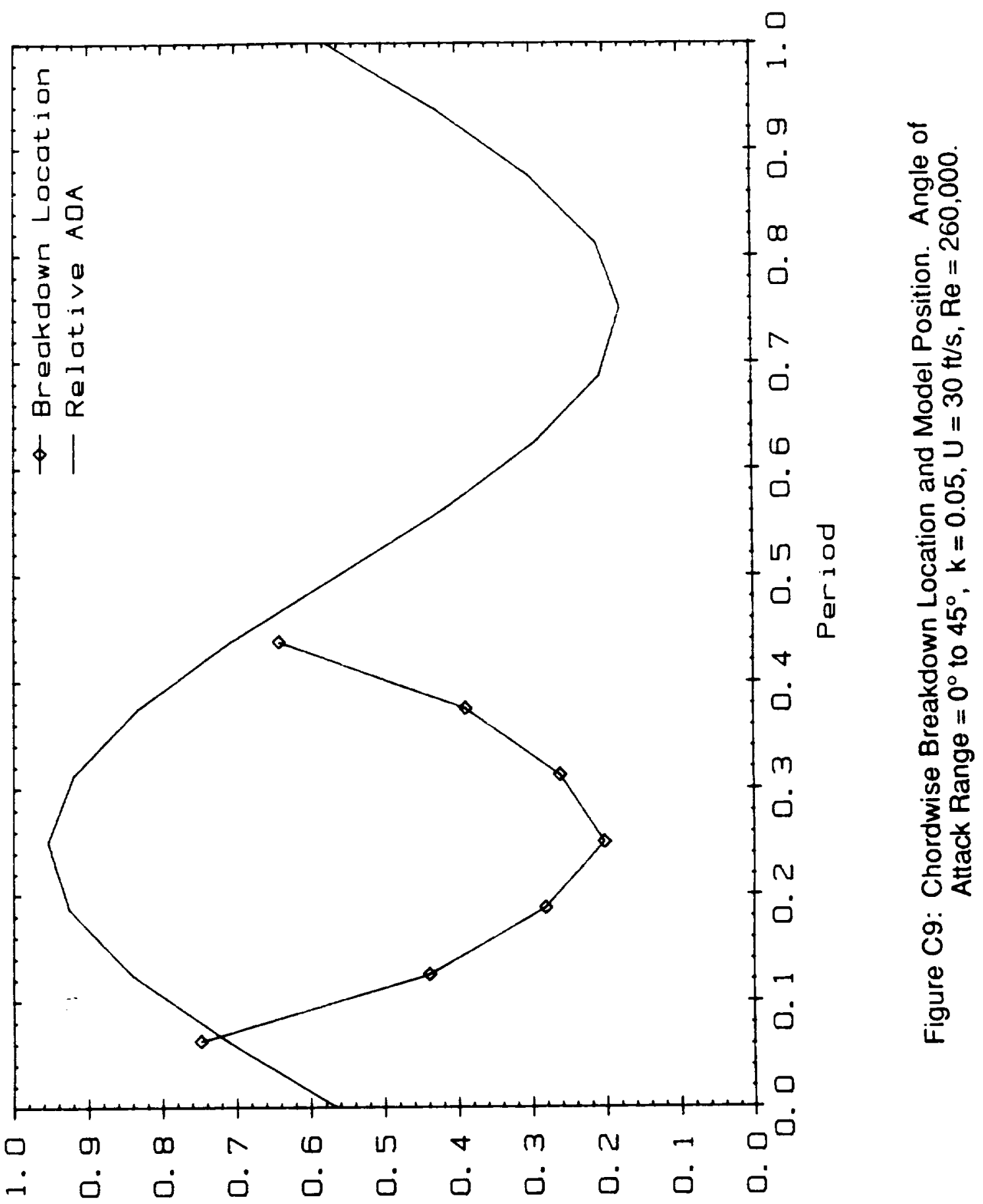

( 


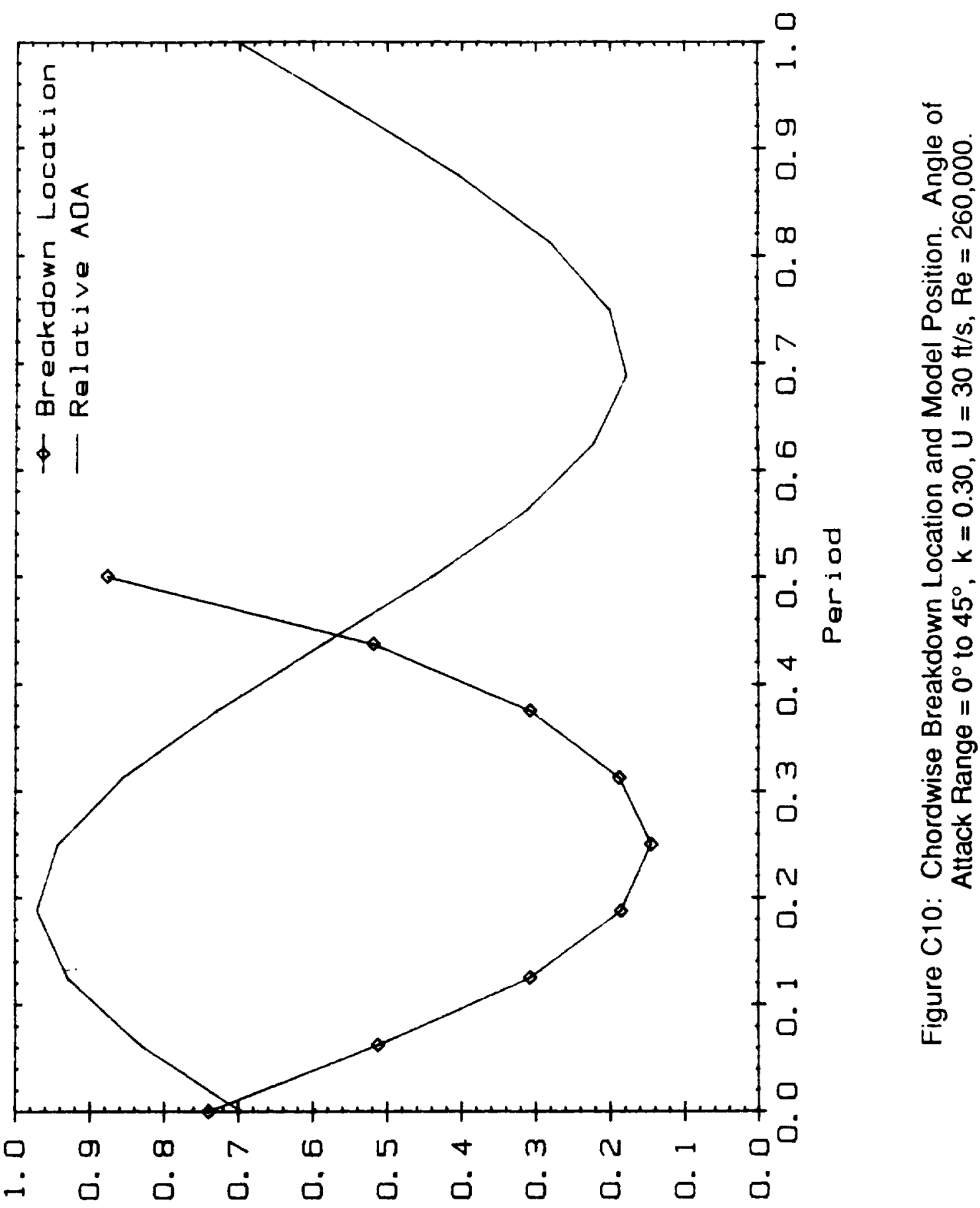

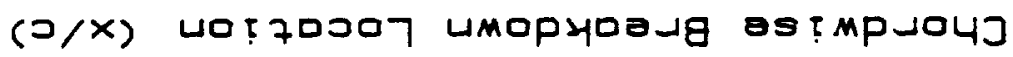




\section{REFERENCES}

Bragg, M. and Soltani, M., "Experimental Measurements on an Oscillating $70^{\circ}$ Delta WIng in Subsonic Flow," Report presented at NASA Lanley Research Center, VA, December 10, 1987.

Corporon, P. G., "Wayne-George Optical Comparator-to-DEC DRV-11 Parallel Line Unit Interface Board User's Manual, University of Notre Dame," June, 1987.

Cunningham, A. M., "Vortex Flow Hysteresis," NASA-CP-2416, No. 11, October 1985.

Elle, B. J., "An Investigation at Low Speed of the Flow Near the Apex of Thin Delta Wings with Sharp Leading Edges," Aeronautical Research Council, $R$ \& M No. 3176, 1958.

Erickson, G. E., "Vortex Flow Correlation," Tech Rept. AFWAL-TR-80-3143, January, 1981.

Gad-el-Hak, M. and Blackwelder, R. F., "The Discrete Vortices from a Delta WIng," AlAA Journal, Volume 23, No. 6, June 1985, p 961.

Gad-el-Hak, M., Ho, C. M., and Blackwelder, R. F., " A Visual Study of a Delta Wing in Steady and Unsteady Motion," Proceedings of the Workshop on Unsteady Separated Flows, editors M. S. Francis and M. W. Luttges, Colorado Springs, Colorado, August 1983.

Gad-el-Hak, M. and Ho. C. M., "The Pitching Delta Wing," AIAA Journal, Volume 23, No.11, 1985, pp1660-1665.

Gilliam, F., Robinson, M., Walker, J. and Wissler, J., "Visualization of Unsteady Separated Flow About a Pitching Delta Wing," AlAA 25th Aerospace Sciences Meeting, Jan 12-15, 1987, Reno Nevada.

Herbst, W. B., "Supermaneuverability," Proceedings of the AFOSR-FJSRLUniversity of Colorado Workshop on Unsteady Separated Flow, USAF Academy, CO, August 1983.

Lambourne, N. C., Bryer, D.W. and Maybrey, J. F. M. "The Behavior of Leading Edge Vortices over a Delta Wing Following a Sudden Change in Incidence," Aeronautical Research Council, R \& M No. 3645, 1969. 
Lambourne, N. C. and Bryer, D. W., "The Bursting of Leading Edge Vortices-Some Observations and Discussion of the Phenomena," Aeronautical Research Council, R \& M No. 3282, April 1961.

Lee, M., Shih, C. and Ho, C. M., "Response of a Delta Wing In Steady and Unsteady Flow," 1987 ASME Applied Mechanics, Bioengineering and Fluid Engineering Conference, Cincinnati, Ohio, June 14-17, 1987.

Lowson, M. V., "Some Experiments with Vortex Breakdown," Journal of the Royal Aeronautical Society, Vol 68, pp. 343-346, 1964.

Malcolm, G. N., "Impact of High-Alpha Aerodynamics on Dynamic Stability Parameters of Aircraft and Missles," AGARD LS No. 114, March 1981.

Malty, R. L., Enger, P. B. and Keating, R. F. A., with Addendum by Moss, G. F., "Some Exploratory Measurements of Leading-Edge Vortex Positions on a Delta Wing Oscillating in Heave," Aeronautical Research Council, R \& M No. 3176, July 1963.

Reynolds, G. A. and Abtahi, A. A., "Instabilities in Leading-Edge Vortex Development," AIAA-87-2424, Applied Aerodynamics and Atmospheric Flight Mechanics Conference, August 17 - 19, 1987.

Rockwell, D., Atta, R., Kuo, C. -H., Hefele, C., Magness, C. and Utsch, T., "On Unsteady Flow Structure from Sweep Eddies Subjected to Controlled Motion," Paper presented at AFOSR Workshop on Unsteady Separated Flows, USAF Academy, CO, July 26 - 30, 1987. 